Portland State University

PDXScholar

$1-1-2012$

\title{
"Man Up": A Longitudinal Evaluation of Adherence to Traditional Masculinity Among Racially/Ethnically Diverse Adolescent Inmates
}

Mary Elisabeth Gray

Portland State University

Follow this and additional works at: https://pdxscholar.library.pdx.edu/open_access_etds Let us know how access to this document benefits you.

Recommended Citation

Gray, Mary Elisabeth, "'Man Up": A Longitudinal Evaluation of Adherence to Traditional Masculinity Among Racially/Ethnically Diverse Adolescent Inmates" (2012). Dissertations and Theses. Paper 106.

https://doi.org/10.15760/etd.106

This Thesis is brought to you for free and open access. It has been accepted for inclusion in Dissertations and Theses by an authorized administrator of PDXScholar. Please contact us if we can make this document more accessible: pdxscholar@pdx.edu. 
"Man Up": A Longitudinal Evaluation of Adherence to Traditional Masculinity Among Racially/Ethnically Diverse Adolescent Inmates

by

Mary Elisabeth Gray

A dissertation submitted in partial fulfillment of the requirements for the degree of

Doctor of Philosophy

in

Applied Psychology

Dissertation Committee:

Eric Mankowski, Chair

Ben Anderson-Nathe

Todd Bodner

Keith Kaufman

Katherine McDonald

Portland State University

(C) 2012 


\begin{abstract}
Research has consistently demonstrated strong relationships between high levels of adherence to traditional masculinity ideology and poor health and behavior outcomes (e.g., Levant \& Richmond, 2007; O’Neil, 2008). Though recent studies have demonstrated support for theories of multiple masculinities or the idea that one's masculinity ideology is developed, maintained, and restructured according to one's social and environmental contexts (e.g., Smiler, 2004), understanding how male gender contributes to social problems within diverse communities, social groups, and contexts is not well established (Mankowski \& Maton, 2010). The current study examined how individual and contextual variables predict change in level of adherence to traditional masculinity ideology among a diverse sample of incarcerated adolescent males convicted of felony crimes in the state of Ohio. In particular, while literature has described prison settings as an environment that ignores gender (e.g., Lutze \& Murphy, 1999; Messerschmidt, 1993), the current study assessed the effectiveness of a strength-based program at successfully decreasing adherence to traditional masculinity within two of the four participating juvenile justice facilities in ODYS. Using hierarchical linear modeling informed by a qualitative follow-up sequence design, study found younger adolescents and African American youth with low levels of ethnic pride to have higher levels of adherence to traditional masculinity at the beginning of the study compared to older adolescents and White youth or African American youth with high levels of ethnic pride. Interestingly, age did not predict changes in levels of adherence to traditional
\end{abstract}


masculinity ideology over time, however, White youth's level of adherence increased over time and African American youth's level of adherence remained relatively stable. Moreover, youth with good attendance in the program experienced less dramatic increases in adherence to traditional masculinity compared to those with poor attendance. Thematic analysis of qualitative data supports the study's finding that program participation predicts changes in levels of adherence to traditional masculinity ideology over time. In addition to providing support for quantitative findings, the thematic analysis highlights some potential gaps in the quantitative assessment of masculinity ideology that must be considered in future research. For example, youth describe an alternative ideal form of masculinity, sometimes characterized by the youth as "man up," that provides a level of flexibility that is counter to that of traditional masculinity. Moreover, the qualitative findings also raise questions about the validity of the survey measure of masculinity (AMIRS; Chu, 2005) for use with African American and incarcerated youth. Finally, the study supports theories of multiple masculinities and offers preliminary evidence that gender specific, strengths-based programming can influence adherence to traditional masculinity ideology among youth in juvenile justice facilities. 


\section{Acknowledgements}

It is a pleasure to thank my committee members for their support, guidance, and inspiration on my dissertation. Most importantly, this dissertation would not have been possible without the support of my advisor and dissertation committee chair, Dr. Eric Mankowski. I am grateful to Dr. Mankowski for allowing me access to and leadership on this large-scale project and for all of his encouragement and feedback along the way. The inspiration for doing longitudinal quantitative modeling for my dissertation came from the teachings of Dr. Todd Bodner. I credit him for both my interest and passion in statistics and am ever grateful for the advocacy and support he has provided me with on this project and during my five years as a doctoral student. The program evaluation aspect of the study was informed through coursework and resources provided by Dr. Katherine McDonald. I am thankful to her for opening my eyes to the world of evaluation research and for all of the advice she provided on this study. I would like to thank Dr. Keith Kaufman for his feedback and support on this project and for the unique insight he provided into the juvenile justice system population. Finally, I would like to thank the graduate studies representative on my dissertation committee, Dr. Ben Anderson-Nathe for his role on this team. It is through the work of Dr. Anderson-Nathe and his colleagues that I was provided with my first glimpse into the hyper-masculine reality of the juvenile justice system.

I would also like to acknowledge Beth Hossfeld and her colleagues from The Council for Boys and Young Men for their collaboration on the larger study in which 
this dissertation is based. I would like to thank the graduate and research assistants in Dr. Eric Mankowski's research lab that assisted with the data collection for the study. Finally, I would like to acknowledge Laura Dolan, the Social Services Administrator for Ohio Department of Youth Services (ODYS), and the youth from ODYS who participated in the study. 
Table of Contents

Abstract

Acknowledgements

List of Tables

List of Figures $\quad$ viii

$\begin{array}{ll}\text { Chapter I: Introduction } & 1\end{array}$

Chapter II: History of Masculinity Theory and Research 8

Chapter III: Masculinity Ideology 30

Chapter IV: Contextualizing Masculinity 44

Chapter V: Development of Research Questions and Hypotheses 85

$\begin{array}{ll}\text { Chapter VI: Methods } & 94\end{array}$

$\begin{array}{lr}\text { Chapter VII: Results } & 128\end{array}$

$\begin{array}{ll}\text { Chapter VIII: Discussion } & 187\end{array}$

$\begin{array}{ll}\text { References } & 262\end{array}$

Appendices

A. Memorandum of Understanding 278

B. Research Protocol 280

$\begin{array}{ll}\text { C. Survey Instrument } & 299\end{array}$ 
List of Tables

Table 1. Masculinity Research Eras 222

Table 2. The Council Curricula: Themes and Activities 223

Table 3. Participants Demographic Information 224

Table 4. Patterning distribution of participants 225 survey responses $(n=1248)$

Table 5. Frequency count of attendance in hours 226

Table 6a. Complete Research Design 227

Table 6b. Research Design of Proposed Dissertation 227

Table 7a. Descriptive Statistics of Continuous Study Variables 228

Table 7b. Descriptive Statistics of Continuous Study Variables 230

Table 8. Time of Measurement Non-Response 231

Table 9a. Predicted Missingness of Age 232

Table 9b. Predicted Missingness of Days in Prison 233

Table 9c. Predicted Missingness of Ethnic Pride 234

Table 9d. Predicted Missingness of Race/Ethnic Identity 235

Table 10. Collinearity Diagnostics of Level-2 Predictor Variable 236

Table 11a. Model Trimming: Complete Model (Model 1) 237

Table 11b. Model Trimming: Model 2

Table 11c. Model Trimming: Model 3

Table 11d. Model Trimming: Model 4

Table 11e. Model Trimming: Model 5

Table 11f. Model Trimming: Model 6 
Table 12. Model Parameter Comparisons Between Samples 244

Table 13. Summary of Hypothesis Testing 245

Table 14. Qualitative Analysis: Phase 1: Data Extract Process 246

Table 15. Qualitative Analysis: Phase 3: Searching for Themes 247

Table 16. Qualitative Analysis: Phase 4: Final Themes and 248 Sub-themes

Table 17. Qualitative Analysis: Data Responses Coded into Eight Themes 
List of Figures

$\begin{array}{ll}\text { Figure 1. Proposed analytic model } & 250\end{array}$

$\begin{array}{ll}\text { Figure 2. Qualitative Analysis: Phase } 2 & 251\end{array}$

Figure 3. Qualitative Analysis: Phase 4

Figure 4. Qualitative Analysis: Phase 5

Figure 5. Control Site Growth Curve (Cuyahoga Hills) 254

Figure 6. Control Site Growth Curve (Indian River) 255

Figure 7. Experimental Site Growth Curve (Circleville) 256

Figure 8. Experimental Site Growth Curve (Ohio River Valley) 257

Figure 9a. Hypothesis 1a: Program Effect 258

Figure 9b. Hypothesis 1b: Dosage Effect 258

Figure 10a. Hypothesis 2a: Age and Initial Levels of Adherence 259

Figure 10b. Hypothesis 2b: Age and Change in Levels of Adherence 259

Figure 11a. Hypothesis 3a: Comparison of significant difference 260 between African American and White

Figure 11b. Hypothesis 4a: Level of Ethnic Pride and Level of Adherence 261 


\section{CHAPTER I}

\section{Introduction}

Over the past decade, "boys" have made national headlines that have identified their gender and age as a social group in a current state of crisis (O'Neil \& Lujan, 2009). Considering the following statistics reported by the Center for Disease Control and Prevention, the "crisis" claim seems to be well-supported. Among 10-24 year olds, boys $(86 \%)$ were much more likely than girls $(14 \%)$ to be victims of homicide (CDC, 2010). Additionally, the majority of youth victims of non-fatal violence are males. Males, ages 10-24, were arrested for violent crimes at a consistently higher rate than females over an 11-year period between 1995 - 2006 (CDC, 2011). Among high school students (grades 9-12), boys reported carrying a weapon $(27 \%)$ or a gun $(9.8 \%)$ during a period of 30-days at a much higher rate than girls $(7.1 \% ; 1.7 \%)(\mathrm{CDC}, 2010)$. There are further disparities among the genders (males $15.1 \%$; females $6.7 \%$ ) on reports of engaging in a physical fight on school property during a one-year period (CDC, 2010). Thus, it is no secret that boys both perpetrate and are victims of violent crime more than girls. In fact, criminologists have consistently used gender, being male, as the strongest predictor of criminal involvement (e.g., Messerschmidt, 1993).

In addition to the gender disparities in violence, recent statistics indicate boys are attaining an education at a lesser rate than girls. For example, in 2003 in the United States the public high school graduation rate was $70 \%$. However, males fell below this average with a $65 \%$ graduation rate, while females exceeded the average 
with $72 \%$ graduation rate (Greene \& Winters, 2006). This gender gap extends to higher education as well, where undergraduate college enrollment is now dominated by women (56\%) and is expected to continue to grow (Freeman, 2006). Despite the support for the claim that boys are in fact in a state of "crisis," an understanding of how male gender roles (i.e., traditional masculinity) contribute to these gender disparities is not well-established (O’Neil \& Lujan, 2009).

Research has recently attempted to answer the question: How do masculine gender roles predict problem behaviors in boys? For example, in an attempt to understand the predictors of school violence, Kimmel and Mahler (2003) argue that masculinity, though often ignored, is the only factor that cuts across all cases of random school shootings in the United States over a 20-year period. Moreover, Blazina, Pisecco, and O’Neil (2005) examined this question among adolescent males ages 13-18 years old and found boys' gender role conflict to predict emotional distress, conduct problems, and poor anger management.

Unfortunately, much of the research conducted with the aim of addressing this question has been limited to homogeneous samples that are made up of mostly White, middle-class males. Given a contemporary and commonly accepted theory of "multiple masculinities," or the idea that one's masculinity ideology is developed, maintained, and restructured according to one's social and environmental contexts (e.g., Smiler, 2004), this research question must continue to be addressed among young men of diverse socio-cultural backgrounds and communities. Clearly, understanding how males' gender contributes to social problems within diverse 
communities, social groups, and contexts is desperately needed (Mankowski \& Maton, 2010).

Overview of the Dissertation Study

The current dissertation examined masculinity ideology among a sample of adolescent males convicted of felony crimes in the state of Ohio and incarcerated in one of four juvenile justice facilities within the Ohio Department of Youth Services (ODYS). Level of adherence to traditional masculinity ideology was assessed on three separate occasions approximately 10-weeks apart. The dissertation examined the effect of a strength-based program, The Council for Boys and Young Men, in successfully decreasing levels of adherence to traditional masculinity ideology. Though it was not assessed directly in the study, given the relationship between high levels of traditional masculinity and poor health and behavioral outcomes that has been established in the literature (e.g., Pleck, Sonenstein, \& Ku, 1993), this dissertation and the program in which the study evaluates took the perspective that high levels of adherence to traditional masculinity ideology is maladaptive in the juvenile justice system. In order to determine the effectiveness of The Council, a non-randomized experimental design was implemented such that two of the four juvenile justice facilities implemented the strength-based program groups into their weekly curriculum, as will be described in greater detail in this dissertation. Due to contextual factors within the two facilities implementing the program, inmates in the experimental locations participated in varied amounts of the program. Thus, the dissertation assessed both a program effect, including participation in the strength- 
based program as a time-invariant predictor, and a dosage effect, including attendance in hours as a time-variant predictor. Moreover, this dissertation examined contextual predictors of change in level of adherence to traditional masculinity ideology. The predictors were measured once (at baseline) along with the first measure of adherence to traditional masculinity ideology before the program was implemented into the curriculum. Finally, this dissertation utilized a qualitative follow-up research design sequence (Morgan, 1998), in which qualitative openended responses were used to support and inform the quantitative findings.

This dissertation used hierarchical level modeling to examine change in adherence to traditional masculinity ideology among incarcerated adolescent males over time. The repeated-measure, which assessed level of adherence to traditional masculinity ideology, served as the Level-1 model in the multilevel model. Given the flexible and dynamic nature of masculinity ideology (e.g., Messerschmidt, 1993), this dissertation also investigated contextual antecedents of change in masculinity ideology. In particular, this dissertation examined whether age, racial/ethnic identity, ethnic pride, and time in prison predicted change in levels of adherence to traditional masculinity ideology, as is highlighted in the theoretical model in Figure 1. The individual-level predictors served as the Level-2 predictors in the multilevel modeling analyses. Additionally, the dissertation examined the effects of a strengthbased program, The Council, at successfully decreasing levels of adherence to traditional masculinity ideology among the experimental group in the study. 
Participation in the program and attendance in the program both served as Level-2 predictors in the multilevel model.

Consistent with research on masculinity ideology among boys and men of different ages (e.g., Cournoyer \& Mahalik, 1995), the dissertation first hypothesized that younger adolescents in the sample will have lower levels of adherence to traditional masculinity ideology, but also experience the greatest amount of change, adhering more to traditional masculinity ideology over time. Second, as several researchers and scholars have previously described (e.g., Franklin, 1984; Levant \& Majors, 1997; Levant, Majors, \& Kelley, 1998), men who identify with different racial/ethnic groups have different levels of adherence to traditional masculinity ideology. Thus, the dissertation investigated the differing levels of adherence to traditional masculinity ideology at baseline (pre-intervention of strength-based program) and the unique changes in adherence over time amongst men of different racial/ethnic identities. Moreover, the dissertation examined whether the level of ethnic pride moderates the relationship between racial/ethnic identity and adherence to traditional masculinity ideology. Third, the environmental context of the prison system has long been associated with increased hegemonic masculine norms (e.g., Jewkes, 2005; Sabo, Kupers, \& London, 2001). For that reason, it was hypothesized that adolescent inmates with greatest time in prison would have high and relatively stable levels of adherence to traditional masculinity ideology, whereas adolescents newer to the prison would have greatest increases in level of adherence beginning at a lower level than those with more experience in the prison. Finally, literature has 
described prison settings as an environment that ignores gender (e.g., Lutze \& Murphy, 1999; Messerschmidt, 1993). However, in the current study, The Council, a strength-based program aimed at promoting healthy masculinities was introduced into two of the four juvenile justice facilities within ODYS in which the study takes place. It was hypothesized that the developmental trajectories of the facilities that participated in The Council would differ from those that did not participate in the program. Specifically, it was hypothesized that the two groups (experimental and control) would begin with the same level of adherence to traditional masculinity ideology and diverge after the introduction of the program, such that the experimental group would have decrease in adherence to traditional masculinity over time, whereas the control group would continue a pattern of increased adherence.

The significant contributions of the dissertation include examination of adherence to masculinity ideology over time amongst adolescent male prison inmates. This work provides a thorough description of the developmental trajectories of adherence to traditional masculinity ideology for adolescent inmates. Additionally, this dissertation assessed antecedents of change in level of adherence among this understudied population. This is a significant contribution to the field, as the lived experiences of inmates in juvenile justice facilities have not been well studied or understood (Cesaroni \& Alvi, 2010). Furthermore, this dissertation has implications for the continued implementation of The Council in the studied juvenile justice facilities and others across the United States. To summarize, these key 
contributions of study provides a unique investigation of the developmental, cultural, and contextual influences on masculinity ideology.

This dissertation is part of a larger study investigating the effects of The Council among a sample of adolescent male inmates within the Ohio Department of Youth Services. This was a collaborative project that included a research team led by Dr. Eric Mankowski, The Council program founder Beth Hossfeld, MFT, and staff at ODYS including Laura Dolan, MSW, LSW, LICDC. I assisted Dr. Mankowski by serving as Project Manager and was involved in all aspects of the project.

In this dissertation I provide an extensive review of masculinity research in the following section, Chapter II. The purpose of this chapter is to outline the history of the field of masculinity research and to describe several conceptualizations and theories of masculinity as a means of providing a foundation of understanding for the literature review to follow. In Chapter III, I describe in greater detail the theory of masculinity ideology, as it is the focus of this dissertation. In this chapter I review the literature on masculinity ideology broadly. The purpose of the chapter to follow, Chapter IV, was to situate masculinity ideology within the context of the current study. Specifically, in Chapter IV, I review literature on adolescent masculinity ideology, ethnic differences in masculinity ideology, and masculinity ideology in prison. Next, in Chapter V, I discuss the current study including the research questions and hypotheses. In Chapter VI, I explain the study context, methods, and analyses. In Chapter VII, I describe the analyses and results of the study. Finally, in Chapter VIII, I discuss the findings, implications and limitations of the dissertation. 


\section{CHAPTER II}

\section{History of Masculinity Theory and Research}

\section{Introduction}

The study of masculinity has taken many different forms over the years. In this section, I describe the important "eras" of masculinity research and present the prominent theories and foundational concepts of each era (see Table 1). The "eras" of masculinity, parallel important eras of United States history, which are represented by social change movements and changes in social climate. I begin with research that was conducted prior to the 1970's and the women's movement, when masculinity was primarily understood in terms of male sex trait theory. Next, I illustrate the research that took place in the 1970's, during the civil rights movement, when masculinity research took on a new form under the androgyny movement, followed by the transcendence and masculinity ideology movements. In the next subsection, I describe the gender role strain, stress, and conflict movements of the 1980's, followed by deconstructionist movement of the 1990's. To conclude this section, I describe the concept of masculinity as it is currently understood.

It is an understatement to say that research on masculinity has grown since the pre-1970's era. To illustrate this growth, I performed a literature search using the keyword "masculinity" and limiting the search to the era's distinguished in this section. During the 1960-1969 (“pre-1970’s”) era, 101 published works were listed; 480 were listed in 1970-1980; 773 in 1980-1989; 972 were listed in 1990-1999; and most recently the largest growth occurred when 2532 works were published in 2000- 
2009. As is highlighted in this basic search of masculinity literature, interest in the topic has grown tremendously over the years. During this time, various theories of masculinity have advanced to the forefront and subsequently faded, as is illustrated in the historical review highlighted in this section.

1900-1970s - Sex-Trait Theory

Prior to the feminist movement of the 1970's, masculinity, as a gender-role, was relatively unstudied. During this time, masculinity was understood as a static trait of a single dimension that opposed the trait of femininity (Smiler, 2004) and was viewed on a single continuum with femininity at one polar extreme and masculinity at the other. In this way, theorists believed that a single individual could possess masculine or feminine qualities, but never both at the same time. During this era, masculinity was viewed as implicit, inherent, and natural, and thus, masculinity was assumed to be culture or context-free (Smiler, 2004). Sex Role Identity (or sex identity), is a term that exemplifies this era and refers to an individual's patterning of sex-typed traits, attitudes, and interests. During this time, the assumed healthy, normal, and ideal state only existed when the patterning of sex-role identity paralleled the individual's biological sex. Though for the most part, theories and understanding of masculinity before the 1970's remained fairly consistent, some change occurred in the theorization of outcomes related to levels of masculinity and femininity post-World War II in the early 1950's. For this reason, I begin this section with a brief discussion on theory, research, and measures that assessed masculinity before the 1950's, and end with a discussion of masculinity in the 1950-1960's. 
Pre-1950's. As noted by several researchers, Terman and Miles are credited for publishing the first psychological measure of masculinity -- the Attitude Interest Analysis Survey -- in 1936 (e.g., Hoffman, 2001; Smiler, 2004). The name Attitude Interest Analysis Survey (AIAS, Terman \& Miles, 1936) was used to conceal its purpose from participants, but is commonly referred to as the MasculinityFemininity test in research circles. The AIAS (or M-F test) is composed of 910 items separated into two equivalent forms (Form A, 456; Form B, 454) (Terman \& Miles, 1936). Consistent with the common beliefs of its time, the scale operationally defined masculinity and femininity as opposing and dichotomous characteristics or aspects of one's personality (Hoffman, 2001; Smiler, 2004; Terman \& Miles, 1936). The survey consists of seven subtests, including: (1) emotional and ethical attitudes; (2) interests; (3) word association; (4) ink-blot association; (5) information; (6) personalities and opinions; and (7) introverted response. The purpose of the scale was to assess differences between one's biological sex and one's "psychological" sex. Pre-World War II theorists tended to view men with high levels of masculine characteristics as acceptable (Smiler, 2004), whereas Post-World War II brought on an era where hypermasculinity was identified as problematic, as it was viewed as related to aggression and delinquency (Pleck, 1987).

By the 1940-1950's, personality inventories were beginning to be developed and utilized at a growing rate (e.g., Ackerman \& Heggestad, 1997). During this time, several personality inventories adopted Terman and Miles' approach to measuring masculinity-femininity (Lippa, 2002). For example, the Guilford and Zimmerman 
(Guilford-Zimmerman scale, 1956) used factor analysis to determine the dimensions of an introversion-extroversion personality scale. One of the statistically determined dimensions was named "masculinity" based on its relatedness to dominance, though it should be noted the authors struggled in naming this factor masculinity, lacking a distinct scale that could assess masculinity sex-role on its own. The GuilfordZimmerman factor that assessed masculinity (and thus, the assumed bipolar opposite of femininity) assessed the control of emotional expression (e.g., inhibited sympathy, display of fearlessness) and male-typical vocational interests (Lippa, 2002).

1950-1960's. Post World War II research on masculinity was focused on the development of sex-roles, particularly in children (e.g., Brown, 1958). As was the case prior to this decade, sex-roles were considered static and dichotomous (male vs. female). Research on sex-role development distinguished between sex-role identification and sex-role preference. Identification referred to how the child was socialized to feel, think, and act like a member of one sex as opposed to the other. Preference referred to the tendency to adhere to the sex-role of one sex in opposition to the other, the adopted sex-role being perceived as more desirable and attractive (Brown, 1958). As is assessed in Terman and Miles' Attitude Interest Analysis Survey (1936), the instruments intended to measure masculinity-femininity focused on the discrepancy between the sex-role identification (i.e., biological sex) and sexrole preference (i.e., psychological sex). The identified patterns of sex-role identification and preference were as follows: (a) identification and preference of the same sex, (b) identification of own sex, preference for other, or (c) identification 
with other sex, preference for own sex (Brown). Normal development was said to occur only when the patterning followed that of the first listed above, when the individual's identified sex matched the individual's preferred sex-role (Brown). The difference noted above between pre-World War II and post-World War II prototypes is evident in the concentration on outcomes theoretically related to sex-role identification, preference and the patterning between the two.

Even before the 1970's feminist movement that made public the power discrepancy between males and females, research had demonstrated masculine role as a privileged sex-role, as compared to the feminine sex-role. In his review, Brown (1958) notes several studies that have found differences in preference of sex-roles between girls and boys. Measuring the level of preference a child expressed towards an image of an object or figure that was typically associated with masculine or feminine roles assessed sex-role preference. Brown noted that boys tended to have a stronger preference for the masculine-role objects and figures, girls for the femininerole objects and figures. The difference in preference was given three different explanations. First, the difference was explained with the Freudian emphasis on the anatomical differences between males and females, where females lack the relevant anatomical part of the males. Second, it was explained by our culture's masculinecentered and masculine-oriented framework that situates the male and masculineroles as superior, and places them in a privileged status. Finally, this difference was explained by the latitude that girls seemed to have in expressing a preference for sex- 
typed objects and activities that was much greater than the latitude accepted for boys (Brown, 1958).

1970 's - Feminist Movement: Androgyny, Transcendence, and Ideology

Bem (1974) critiqued pre-1970's sex-role theory for dichotomizing masculinity with femininity and not accounting for individuals with "androgynous" qualities. Additionally, Bem critiqued sex-role theory for not taking situational factors into account, thus assuming sex-roles to consist of static traits inherent to the individual. She posited that some individuals may possess both masculine and feminine qualities, for example, "both assertive and yielding, both instrumental and expressive -- depending on the situational appropriateness of these various behaviors" (Bem, p. 155). This points to her second critique that situations may elicit, allow for, or be most responsive to certain sex-role characteristics. In response to these critiques, Bem proposed the concept of sex-role androgyny.

Using the Bem Sex-Role Inventory (BSRI; Bem, 1974), Bem was able to assess her theory of androgyny by including both a Masculinity Scale and a Femininity Scale. Each scale listed 20 masculine or feminine characteristics as well as an additional 20 neutral (unassigned to a gender) characteristics. For example, masculinity was assessed with items such as, "aggressive, athletic, dominant, and self-reliant"; femininity was assessed with items such as, "cheerful, gentle, loyal, and soft spoken." Items such as "adaptable, friendly, sincere, and tactful" were listed as gender-neutral. The BSRI characterized a person to be masculine, feminine, or androgynous as a function of their combined level of endorsement of feminine and 
masculine items. The 20 items that were intended to be gender neutral served as an assessment of social desirability bias because it would allow the researcher to determine whether the participant was tending to respond to items that described socially desirable traits. In other words, the neutral items did not count towards a score of masculine, feminine or androgynous. The scores on each of the ten items (masculine and feminine) were summed separately to determine the degree of masculinity or femininity of a respondent. To determine how androgynous a respondent was, these two scores were added together and multiplied by two. An individual with high scores on both masculine and feminine items is considered highly androgynous.

The intention of the BSRI was to assess androgyny, or the individual's flexibility of their sex-role. Androgyny theories eliminated the single dimension bipolar assumption of masculinity as opposite to femininity and promoted sex-role flexibility as desirable (Smiler, 2004). In her paper, Bem (1974) theorized that not only was sex-role rigidity an outdated concept, but sex-role flexibility (a.k.a. androgyny) would come to be defined as the standard for good health.

Stemming from critiques of the androgyny movement, the sex-role transcendence theory emerged in the mid-1970's and was believed to be the ideal state of sex role development at that time (e.g., Garnets \& Pleck, 1979). Sex-role transcendence refers to "a stage in which masculinity and femininity are 'transcended' as ways of organizing and experiencing psychological traits” (Garnets \& Pleck, p. 273). Unlike androgynous and sex-role identity theorists, transcendence 
theorists did not attempt to link adherence to sex-role related traits with psychological well-being or adjustment (Garnets \& Pleck). A major limitation of the theory of transcendence is that it is extremely difficult operationally define the theory and research or test the validity of it.

By the mid 1970's, the Masculinity Ideology or Belief movement began to take shape. Masculinity ideology differed from the androgyny and transcendence theories in that masculinity was no longer viewed as an inherent or acquired trait, rather as a social ideal in which individuals attempt to conform (Smiler, 2004). Brannon first provided a description of four social themes regarding traditional mainstream U.S. cultural ideals of masculinity in 1976, and in various later forms (Brannon, 1985). The four themes include: (1) anti-femininity; (2) status and achievement; (3) inexpressiveness and independence; and (4) adventurous and aggressive. Anti-femininity or, in other words, "No Sissy Stuff," refers to the avoidance of behaviors, interests or personality traits that are considered feminine. This masculine ideal is encouraged in boys at a young age. Definitions of these "feminine" qualities are determined by the mainstream culture's (United States) view and definition of femininity and masculinity. For example, Kilmartin (2007) describes current mainstream American ideas of feminine qualities as "gentle, sensual, tender, submissive, passive, relationship-oriented, and sexually desirous towards males" p. 204-205. Thus, under this theme, men should try to avoid expressing all of those qualities. Of these four traditional masculine "norms" or ideals, anti-femininity is believed to be the central factor from which all of the other 
norms are derived (Brannon, 1985). Status and achievement or "The Big Wheel" refers to the expectation of men to be successful and powerful. Specifically, traditional masculinity emphasizes the outcome of success, not the process or experience of doing something, and typically relies on obvious and transparent quantifications that clearly identify success (Kilmartin). Success can refer to a man's success in sports and work, but also notably to sexual success, signified by a man's ability to produce an erection at will, his sexual stamina, and his ability to give his female partner multiple orgasms (Kilmartin). Inexpressiveness and independence or "The Sturdy Oak," refers to upholding emotional control and self-reliance even in the most challenging situations. In mainstream United States culture, men are expected to know without being told, and tend to rely on peer groups for transmitting (mis)information (Kilmartin). Finally, Adventurousness and aggression or "Give 'em Hell," refers to the expectation of a man to be fearless, brave, and self-assertive. This masculine ideal is also characterized by a man's willingness to take physical risks. Under this lens, men are expected to exercise dominance and control in all areas, including in sexual relations.

1980 's - Hegemonic Masculinity -- Gender Role Strain, Stress, and Conflict

The concept of hegemonic masculinity was formulated in the early 1980's and has considerable influence in theory about men, gender, and social power hierarchy (Connell \& Messerschmidt, 2005). Hegemonic masculinity refers to the pattern of gender-role expectations, identity, or behaviors that allow for a continued male dominance over females (Connell \& Messerschmidt). Hegemonic masculinity 
takes up where Brannon left off in describing a culturally normative ideal of male behavior. Additionally, Brannon and other researchers and theorists have continued to describe themes of traditional masculinity. Unfortunately, however, what researchers have determined during this era is that adherence to hegemonic masculinity or traditional masculinity ideology (e.g., Brannon's four themes) is not always adaptive or natural. Based on theories from the men's liberation movement and the psychology of women, research in the early 1980's established justification for studying the conflict, strain, or stress surrounding mens' gender role socialization based on traditional or hegemonic masculine ideals (O’Neil, 2008). Broadly speaking, gender (or sex) role conflict, strain, or stress refers to the psychological situation that occurs when gender role demands or ideals have negative consequences for the individual or others, or when they conflict with naturally occurring tendencies within the individual (Kilmartin, 2007). In the paragraphs to follow I describe the gender role strain and conflict era in order of their appearance in the literature: First, I introduce Garnets and Pleck's (1979) sex-role strain analysis; Second, I describe O’Neil and colleagues (1981) gender-role conflict model; Finally, I discuss Eisler and Skidmore’s (1987) masculine gender role stress model.

In 1979, Garnets and Pleck introduced the sex-role strain analysis, which described the relationship between sex-role related personality characteristics and psychological adjustment. The concept of sex-role strain is rooted in the theory of self-discrepancy. Self-discrepancy theory describes chronic discrepancies between one's self-concept and self-guides, where it is assumed that people are motivated to 
reach a condition where their self-concept and self-guide match (Higgins, 1987). Specifically, self-discrepancy theory assumes three domains of the self: (1) the actual self, attributes the individual believe s/he actually posses; (2) the ideal self, attributes the individual would like to possess; and (3) the ought-self, attributes the individual believes s/he ought to possess. Ultimately, the theory of self-discrepancy proposes that inconsistencies in the abovementioned self-states result in negative emotions. More specifically, discrepancy is believed to lead to two distinct types of negative physiological situations - dejection-related emotions associated with the absence of positive outcomes and agitated-related emotions associated with the presence of negative emotions. In a similar manner, the sex-role strain model posits that the relationship between sex-typing and adjustment is moderated by two variables: (1) the individual's ideal for his sex; and (2) the degree of salience from the individual's sex role. Sex-role strain is operationally defined with three variables: (1) real self-concept; (2) same-sex ideal; and (3) sex-role salience. Real self-concept refers to the sex-role characteristics in which an individual perceives to actually posses. This variable may be measured using self-ratings of masculinity and femininity (e.g., Bem Sex Role Inventory, Bem, 1974). Classifications of real selfconcept include sex-typed such that self-concept matches biological sex or androgy. Same-sex ideal refers to the sex-role characteristics, which individuals perceive that members of their own sex ought to possess. Classifications for same-sex ideal are equivalent to that of self-concept, including sex-typed and androgy. Sex-role salience refers to the degree to which individuals organize personality characteristics into 
categories of masculinity and femininity, in which they psychologically orient themselves. For example, low sex-role salience may be demonstrated in a man who is self-reliant and who as a result perceives himself to be a self-reliant person, whereas high sex-role salience may be demonstrated in a man who is self-reliant and as a result experiences himself as masculine.

Outcomes of the sex-role strain model are determined by the discrepancy between the real self-concept and that part of the ideal self-concept that is culturally associated with gender (Garnets \& Pleck, 1979). If the discrepancy between the real self-concept and ideal self-concept is large, the result is high sex-role strain.

However, because salience plays a moderating role in this model, the relationship depends on the degree of salience. For low salient individuals, the relationship between the discrepancy and sex-role strain is reduced. For high salient individuals, the relationship between discrepancy and sex-role strain is heightened. In the sexrole strain model, there are five distinct sex-role strain outcomes: (1) low sex-role strain (with sex-typed same-sex ideal); (2) high sex role strain (with sex-typed samesex ideal); (3) low sex -ole strain (with androgynous same-sex ideal); (4) high sexrole strain (with androgynous same-sex ideal); and (5) low sex-role strain (with low sex-role salience). Thus, healthy masculinity according the sex-role strain model would occur when sex-type and sex-ideal are closely aligned.

Stemming from Garnets and Pleck's (1979) theory of sex-role strain, O’Neil (1981) proposed The Gender Role Conflict (GRC) Model that hypothesizes six theoretical factors of GRC related to mens' gender role socialization and the 
masculine norm that is based in fear of femininity (anti-femininity; Brannon, 1985). Gender role conflict refers to "a psychological state in which socialized gender roles have negative consequences for the person and others" (O’Neil, 2008, p. 362). Specifically, negative consequences are believed to occur when rigid and restrictive gender role norms (e.g., anti-femininity, emotional control) result in the deprecation or limitation of others or self. The six hypothesized patterns of GRC include: (1) restrictive emotionality; (2) health care problems; (3) obsession with achievement and success; (4) restrictive sexual and affectionate behavior; (5) socialized control, power, and competition issues; and (6) homophobia. In order to statistically assess the underlying structure of the Gender Role Conflict Model, the Gender Role Conflict Scale (GRCS; O’Neil et al., 1986) was developed based on psychometric analysis and subsequently used in over 230 studies since the mid-1970's (O'Neil, 2008). Studies examining gender role conflict will be reviewed in detail in the chapters to follow.

Currently, gender role conflict is operationally defined with four psychological factors, several situational contexts, and three personal experiences resulting from the individual interaction with masculinity ideology (O’Neil, 2008). Broadly speaking, psychological factors describe the processes in which an individual interacts with established gender role norms, situational contexts refer to the environmental influences affecting an individual's interaction with gender norms, and personal experiences describe the result of the pathway chosen by the individual to interact with or avoid adhering to their gender role norm. In the three paragraphs 
to follow, I will describe in detail the psychological factors, the situational contexts, and discuss the personal experiences that define gender role conflict.

The four psychological factors of gender role conflict are: (1) cognitive; (2) affective; (3) unconscious; or (4) behavioral processes (O’Neil, 2008). Each of the four psychological factors are said to be caused by a socialization process in which an individual learns his/her gender role in societies that are sexist and patriarchal (O’Neil), such as United States mainstream culture. Cognitive processes refer to how an individual thinks about gender roles. Affective processes refer to how an individual feels about gender roles. Unconscious processes refer to how gender role dynamics function beyond the individual's awareness. Behavioral processes refer to how an individual acts, responds to, or interacts with others or with themselves because of gender roles.

Situational contexts that affect gender role conflict are complex and varied. O’Neil (1990) reduced the complexity of the range of possible situational contexts the four categories: (1) GRC caused by gender role transition; (2) GRC experienced intra-personally (within the individual man); (3) GRC expressed toward others interpersonally; and (4) GRC experienced from others. Gender role transitions refer to events that disrupt an individual's gender role development such that it alters or challenges his gender-role self-assumptions, resulting in the production of GRC, or alternatively, positive life changes (O’Neil, 2008). For example, gender role transitions may occur when a man enters the workforce, gets married, or has children. Intra-personal contexts refer to a private experience of negative emotions 
and thoughts resulting from one of the three personal experiences listed and described in detail below. On the other hand, interpersonal contexts refer to the result of the personal experience that is expressed outwardly toward other people. Finally, GRC from others occurs when someone else harms another person who deviates from or conforms to traditional masculinity ideology and norms (O’Neil, 2008).

The three personal experiences represent the range of possible negative consequences that may results when conforming to, deviating from, or violating the masculinity ideology norms (O’Neil, 2008). The first personal experience is devaluation, referring to the negative critiques of self or others as the result of not conforming to or deviating from traditional masculine norms. Restriction refers to the constraint of oneself or others in order to adhere to traditional masculinity ideology. Violations refer to hurting oneself or others, or being harmed by others, as a result of conforming to or deviating from traditional masculine norms. The result of devaluation is a decrease in social status, the result of restriction is the controlling of other's behavior or limiting one's own potential or flexibility, and the result of violation is victimization or abuse, causing physical or psychological pain.

Taken together the GRC occurs when rigid or restrictive gender roles promote the devaluation, violation, or limiting of oneself or others (O’Neil, 2008). Ultimately, the GRC model explains how stringent gender roles may restrict one's potential or the potential of others around the individual. The implications of the GRC model are to restrict gender roles are engrained via psychological, situational, 
and personal pathways. These deeply engrained gender roles are understood to be harmful when they are restrictive and rigid. Although GRC is not assessed directly in the current study, adherence to traditional masculine norms is. Because traditional masculine norms are restrictive and rigid, it is implied that blind adherence to these traditional masculine norms may be maladaptive and unhealthy in some situations.

In 1987, Eisler and Skidmore proposed a theory of masculine gender role stress. Masculine gender role stress refers to the "cognitive appraisal of specific situations as stressful for men" (Eisler \& Skidmore, 1987, p. 125). The stressful situation takes into account three variables: (1) cognitive: the individual's thoughts; (2) behavioral: the individual's behaviors; (3) environmental: the environmental context or events. Based on the previously examined definition of hegemonic masculinity (Connell \& Messerschmidt, 2005) or traditional masculinity ideology, stress will result when a man perceives himself to be unable to cope with the ideals of the male role or when a situation is viewed as requiring feminine (a.k.a. "unmanly") behavior (Eisler \& Skidmore). Results from a factor analysis on the 40item scale of masculine gender role stress displayed five distinct domains. The results suggest that men are prone to stress in situations that reflect the following: (1) physical inadequacy; (2) emotional inexpressiveness with regard to the more tender emotions; (3) subordination to women, involving situations in which women are dominant, in charge, or more successful; (4) feeling intellectually inferior, which threatens the individual's perceptions of control and rational decisiveness; and (5) performance failures in work or sex (Eisler \& Skidmore, 1987). 
Gender (or sex) role strain, conflict, and stress each concern ways in which men (or women) may respond in particular situations. However, they differ in that gender role strain and conflict refer to a restriction of human potential, whereas stress refers to a feeling or emotion. As Smiler (2004) points out however, "perpetual role stress could lead to the restriction of human potential" (p. 19). Thus, the constructs may be linked within an individual. The measures used to assess gender role conflict and masculine gender role stress each have identified factors or subscales, including some overlapping concepts (e.g., anti-femininity). For both measures, lower scores are more desirable. This "ideal" differs from that of the previous eras in that problems previously associated with being or acting hypoand/or hyper-masculine are now related to fulfilling the masculine role (Smiler, 2004).

By the end of the 1980's, theorists began to criticize literature on gender for ignoring contextual and sociocultural influences in its research (Smiler, 2004). Critiques similar to the one being made in gender research were being made in other fields, specifically Community Psychology (e.g., Trickett, 1996), and theorists argued for researchers to adhere to interactional or transactional worldviews (Altman \& Rogoff, 1987) in their work. In response, some researchers began to consider the external influences on gender ideology before the 1990's (e.g., Pleck, 1987). However, the implementation of interactional or transactional worldviews in gender research did not make large advances until the 1990's.

$$
1990 \text { 's - Deconstructionist Movement }
$$


The 1990's began the contextualized masculinity era that continues to exist today. In the deconstructionist movement, researchers and theorists for the first time collectively agreed that masculinity may take on multiple forms for an individual depending on the context they are in or their sociocultural influences. In contextualizing masculinity, the idea that masculinity was something that resided within the individual was challenged for the first time (Smiler, 2004).

The deconstructionist movement maintains the idea that a common masculinity ideology exists, such that traits, attributes, and characteristics are considered normal masculine tendencies, though variations in what is considered the ideal have occurred over time (Smiler, 2004). Though the masculinity ideology movement of the 1970's did not discuss variation in adherence to masculine norms, masculinity ideology is understood to be diverse and variable today (Smiler, 2004). Additionally, whereas previous theories viewed hypo- or hyper-masculinity as problematic, the current era views the act of insufficiently or overly supporting specific elements of masculinity ideology as problematic (Smiler). To be clear, previous theories related problem outcomes with internal masculinity, whereas current theories relate problem (or positive) outcomes with an individual's performance of or adherence to an external socially constructed variable of masculinity. Though some theorists (e.g., Pleck; Wade) continue to believe that masculinity resides within the individual, these theorists believe that the construct of masculinity is externally and socially defined and altered by the social setting or context - demographic group or reference group (Smiler, 2004). To illustrate, 
multiple patterns of masculinity have been identified in several studies across a variety of cultures and contextual settings (Connell \& Messerschmidt, 2005). For this reason, theorists now believe that multiple masculinities are present and a specific form or level of masculinity is no longer specified as ideal (Smiler, 2004). One common way in which multiple masculinities has been demonstrated in research has been through the description of masculine forms within demographically defined groups such as: homosexuals (e.g., Connell, 1992), African Americans (e.g., Levant, Majors, \& Kelley, 1998; Wade, 2008a), Latinos (e.g., Saez, Casado, \& Wade, 2009), and boys and men of different age groups (e.g., Cournoyer \& Mahalik, 1995). The problematic outcomes previously related to a single concept of masculinity are no longer believed to be correlated with the "possession" of masculinity, but the lack of behavioral flexibility in a given ideology (Smiler, 2004). In this way, current theories and research parallel Bem's ideals of flexible sex-roles, including those who have observed that overly rigid adherence to masculine norms is problematic (e.g., Wade, 2008b).

Current theories of masculinity neglect to include femininity in their definition (Smiler, 2004). This lack of consideration of femininity is different from that of historically popular theories where femininity was viewed as opposite and/or completely independent of masculinity. On the other hand, similar to masculinity ideology definitions from previous eras (e.g., Brannon, 1976), masculinity continues to be viewed as having distinct components (Smiler, 2004). 
A contemporary notion of traditional masculinity ideology or hegemonic masculinity may be best described by Pollack (2000), who has longitudinally studied boys and young men as an attempt to better understand masculinity. Pollack (1999, 2006a) describes a current male-based socialization code, referred to as boy code, a concept that is created and sustained by American culture in which a boy is shamed into extreme behaviors that fit one traditional norm of masculinity (i.e., selfcontainment, toughness, and separation). Additionally, Pollack has described a contemporary traditional masculinity model to include a development of separation, autonomy, and individualistic coping for boys as young as 3-5 years, which results in an early silencing of expression, emotion, and vulnerability (Pollack, 2006a). To take this contemporary notion of masculinity ideology into imagery, Kivel (2007) describes the "Act-Like-A-Man" Box, in which ideals of traditional masculinity are contained within the box and physical and verbal abuse tactics are situated around the box. This image may be interpreted by the idea that men are constrained within a box, such that in the event that they step out they will experience negative feedback from their social system for their lack of conformity to masculine norms.

\section{Conclusion}

As is described above, the evolution of the study and understanding of masculinity has taken many different forms (Smiler, 2004). Prior to the 1970's and the women's movement and feminist critique, masculinity was primarily understood in terms of male sex-role theory. Under this theory, masculinity is seen as a single, unidimensional construct in which males attempt to acquire attributes that are 
considered masculine to affirm their biological identity of being male. During this time, masculinity was seen as the polar opposite to femininity: low levels of masculinity and in turn, high levels of femininity in men, were seen as problematic. In the 1970 's, masculinity research took on a new form under the androgyny movement. Androgyny theories eliminated the bipolar assumption of masculinity as opposite to femininity and promoted sex-role flexibility as desirable. By the mid 1970's, the Masculinity Ideology movement began to take form, with Brannon (1976) describing the underlying structure of masculinity. Masculinity ideology differed from the androgyny theories in that masculinity was no longer viewed as an inherent or acquired trait, but rather as a social role in which individuals attempt to conform. The 1980s were met with the gender role strain, conflict, and stress movements, in which the attempt to conform to a gender role was theorized to be met with difficulties because the dominant masculine ideals included some dysfunctional elements. By the 1990s, researchers and theorists in the field began to take social and historical contexts into account, following a deconstructionist movement. In contrast to the ideology perspective, which does not discuss individual variation, masculinity theories under this movement highlight variations in masculinity between social contexts and over time. Current movements have updated existing theories of masculinity role, identity and ideology to incorporate the idea of multiple masculinities and have addressed other concerns, including the rigidity and unidimensionality of previous conceptualizations of masculinity raised by critics during the various movements over the past 40 years. Collectively, researchers and 
theorists now tend to describe masculinity as an internalized construct with a few underlying factors, one of which typically opposes femininity (Smiler, 2004).

Moreover, masculinity is understood in terms of social, sociocultural, and historical influences.

Now that the history of masculinity research and theory has been described in detail, I will apply this foundation to the present study. First, I provide a detailed review of masculinity ideology research in Chapter III. To follow, I contextualize the review of literature to fit the purposes of the current study in Chapter I 


\title{
CHAPTER III
}

\author{
Masculinity Ideology
}

\section{Introduction}

As highlighted in Chapter II, the study and theory of masculinity as a psychological construct has evolved over the years. Although the construct of masculinity ideology was first proposed in the 1970's (e.g., Brannon, 1976), the current conceptualization of this construct has changed in ways that parallel historical social movements and psychology's zeitgeist previously described. Building on the background provided in chapter II, in the current chapter I define masculinity ideology, briefly outline the history of this construct, describe the most commonly used measures that assess masculinity ideology, and conclude with a general overview of the literature on masculinity ideology. In presenting the various masculinity ideology measures available in current literature, I justify the use of the Adolescent Masculinity Ideology in Relationship Scale (Chu et al., 2005) in the current study. In the Chapter to follow, Chapter IV, I provide a more detailed review of the literature on masculinity ideology within the specific context of the current study.

\section{Masculinity Ideology}

The goal of this dissertation is to better understand how adherence to traditional masculinity ideology is affected by social and environmental contexts. Specifically, this dissertation proposes to examine the developmental trajectory of adherence to traditional masculinity ideology among incarcerated adolescent males. 
"Masculinity ideology can be defined as an individual's internalization of cultural belief systems and attitudes toward masculinity and men's roles. It informs expectations for boys and men to conform to certain socially sanctioned masculine behaviors and to avoid certain proscribed behaviors" (Levant \& Richmond, 2007, p. 131). Thompson and Pleck (1995) distinguished gender orientation from gender ideology in describing two different approaches to studying masculinity: (1) trait perspective; and (2) normative perspective. The trait perspective assumes masculinity to be rooted in the sex of a person and, as such, masculinity is studied as an aspect of men's personality (e.g., Spence \& Helmrich's 1978 Personal Attributes Questionnaire) or behavioral attributes (e.g., Snell's 1989 Masculine Behavior scale). The trait perspective, as outlined in Chapter II, was dominant in research and theories of masculinity and gender before the deconstructionist movement took hold in the 1990's. Men who possess particular attributes or behavioral tendencies were said to be inherently more "masculine" than those who do not. Normative perspectives, on the other hand, are located outside the individual such that masculinity is a component of a broader gender ideology with scripted values, traits, and behaviors. For example, a traditional man would endorse the idea that men should have sex-specific characteristics, but that women should not. Masculinity ideology in the current study is defined using the normative perspective.

As is common in contemporary theory of the deconstructionist movement, masculinity ideology is currently defined such that it allows for multiple forms of masculinity to exist. Thompson and Pleck (1995) argue that a single standard of 
masculinity ideology is apolitical and ignores the imbalance of power between men and women (Connell, 1987). In fact, the variation in responses to masculinity ideology (e.g., by ethnicity, age, geographic location), not only highlights the idea of multiple masculinities, but also brings light to the differences in masculinity ideology that are present in different contexts. For example, researchers have demonstrated that masculinity ideology differs across racial-ethnic groups (Cazenave \& Leon, 1987; Franklin, 1988) and across different generations (Cournoyer \& Mahalik, 1995). Moreover, masculine norms within juvenile justice facilities have been described to be different from those outside (e.g., Abrams, Anderson-Nathe, Aguilar, 2008).

Despite the fact that masculinity ideology is understood to have diverse and variant content, there exists a common set of standards and expectations that are associated with the traditional male role, referred to as the traditional masculinity ideology by some (e.g., Pleck, 1995) or hegemonic masculinity by others (e.g., Connell, 1995) (Levant \& Richmond, 2007). The current study is based on the traditional masculinity ideology (or hegemonic masculinity) defined by Chu, Porche, and Tolman (2005), which include emotional stoicism, heterosexual dominance, sexual "drive", physical toughness, competitiveness, and ambition. Several masculinity theorists and researchers have identified themes of traditional masculinity common to the Western World. For the most part, these themes parallel each other. For example, Chu and colleagues definition of traditional masculine ideals parallel Brannon's (1976) four themes. Recall from Chapter II that Brannon 
described themes of traditional masculinity in the following ways: (1) inexpressiveness; (2) anti-femininity; (3) adventurous and aggression; (4) status and achievement. Additionally, in 1995, Doyle added a fifth dimension, sexual, to Brannon's themes. According to Doyle (1995), sexual refers to the idea that men should always be ready and willing to have sex with women. Chu and colleague's description of traditional masculine ideals parallel that of Brannon and Doyle in the following ways: (1) Emotional Stoicism $(\mathrm{Chu}$ et al. $)=$ Inexpressiveness $($ Brannon); (2) Heterosexual Dominance = Anti-Femininity; (3) Physical Toughness = Adventurous and Aggression; (4) Competitiveness and Ambition = Status and Achievement; (5) Sexual Drive = Sexual (Doyle). Although, as is illustrated above, masculinity ideology may take many forms, there remain several dimensions of traditional masculinity that have been consistently identified in the literature. Assessing Masculinity Ideology in Research

Over the past three decades, researchers have developed several scales to assess an individual's level of adherence to traditional masculinity ideology. In order to thoroughly understand the research on masculinity ideology, one must also understand the survey instruments used to assess this construct. The studies that are reviewed in the current chapter and the contextual chapter (Chapter IV) to follow utilize the measures described in this section. Thompson and Pleck describe two general types of research based on masculinity: (1) research that assesses men's experiences (usually negative) with their gender-related beliefs; and (2) research that assesses mens' level of adherence to traditional masculinity ideologies. For the 
purpose of this study, this section will focus on the latter - research to assess adherence to traditional masculinity ideologies. The perspective taken in this dissertation is consistent with current theoretical perspectives that the act of overly support ideals that are consistent with traditional masculinity are maladaptive and related to poor health and problem behavior. In the paragraphs to follow, I present the most commonly used measures of masculinity ideology in the order they are presented in the literature with a focus on those utilized in the review of masculinity ideology literature at the end of the chapter.

Masculinity ideology was first assessed using Brannon's Masculinity Scale (BMS; Brannon \& Juni, 1984), which is a 110-item scale $(\alpha=.95)$ assessing normative statements of traditional masculinity. The BMS was based on Brannon's (1976) four-theme's representing American cultural ideal of masculinity described in detail in chapter II. The first theme, Anti-femininity was assessed with two 16-item subscales: (1) avoiding feminine behavior; and (2) concealing emotion. Achievement and status was assessed with 15-items regarding admiration for success and family breadwinner status. Inexpressiveness and independence was assessed with two 16item subscales: (1) toughness; and (2) male machine. Finally, adventurous and aggression were assessed with 15-items representing adventure and bravado. Taken together, a composite score on the BMS represents an individual's endorsement of traditional masculinity ideology. The strength of the BMS is the large scope of masculine standards assessed. Nevertheless the BMS has several noted limitations, including: Its length (time consuming) and its lack of attention towards male 
privilege, male rights, and male sexuality (Thompson \& Pleck, 1995). The BMS is not utilized in the current study due to its limitations in length (overburden to the participant and administrators of the survey) and because it has typically been assessed with adult populations.

In 1981, O’Neil and colleagues published the Gender Role Conflict Scale, and, in 1987, Eisler and Skidmore published the Masculine Gender Role Stress scale, both of which were reviewed in detail in chapter II. In between the publishing of these two scales, Thompson and Pleck (1986) published the Male Role Norms Scale (MRNS). The MRNS is a 26-item inventory used to assess traditional masculinity ideology. The MRNS was derived through a factor analysis of the BMS which was reduced to three basic dimensions: (1) status norms (11 items, $\alpha=.81$ ); (2) toughness norms (8-items, $\alpha=.74)$; and (3) anti-femininity norms (7-items, $\alpha=.76$ ). Similar to the BMS, the MRNS did not contain items regarding attitudes towards women or gender in general. The strengths of the MRNS are its brevity and its strong evidence of construct and discriminant validity. The MRNS is limited by its reliance on one version of masculinity and the lack of attention towards male privilege (Thompson \& Pleck, 1995). Like the BMS, the MRNS has not established reliability and validity among an adolescent sample, thus, this scale is not be assessed in the current study.

In 1986, Snell, Belk, and Hawkins (1986) published the 60-item Stereotypes About Male Sexuality Scale (SAMSS) to assess 10 stereotypes about male sexuality: (1) men should not have certain feelings; (2) sex equals performance; (3) men must 
orchestrate sex; (4) men are always ready for sex; (5) all physical contact leads to sex; (6) sex requires erection; (7) sex equals intercourse; (8) sex requires orgasm; (9) sex is spontaneous; and (10) men are sexually knowledgeable. Each of the 10 stereotypes are evaluated using six declarative (some prescriptive, some descriptive) statements about men.

Snell published a different measure of masculinity, The Masculine Behavior Scale (MBS) in 1989. The MBS consists of first-person belief statements regarding traditionally stereotypical masculine behaviors. This scale is made up of four subscales that were identified statistically through factor analysis: (1) success dedication; (2) restrictive emotionality; (3) inhibited affection; and (4) exaggerated self-reliance. Respondents are asked to indicate their level of agreement on a fivepoint Likert scale, ranging from +2 to -2 . Higher scores on the MBS indicate greater adherence to traditional views of mens' expected behavior. Snell has demonstrated adequate to strong reliability ( $\alpha=.69-.89$ ) in each of the subscales of the MBS for a population of undergraduate students in the Midwest. The current study assesses masculinity ideology among a range of other measurements included for the purposes of a larger study. Additionally, due to Ohio Department of Youth Services staff's concerns of illiteracy among the adolescent inmates, the surveys were orally administered in a group setting. For these reasons scale brevity is an important attribute in assessing this construct. Therefore, the MBS was not utilized in the current study. 
In 1992, Levant and colleagues criticized the redundancy and overlap among the subscales of the BMS and in response developed the Male Role Norms Inventory (MRNI) to assess both traditional and non-traditional masculinity ideologies. Since the MRNI was published in its original form, new versions have been created, including the MRNI-A, a 43-item version designed for adolescents. The original scale assess seven theorized normative masculine standards: (1) avoidance of femininity (8-items); (2) homophobia (5-items); (3) achievement/status (10-items); (4) attitudes towards sex (10-items); (5) restrictive emotionality (10-items); (6) selfreliance (7-items); and (7) aggression (8-items). Three statistical underlying dimensions determined via confirmatory factor analysis were determined: (1) items from the femininity avoidance, homophobia, achievement/status, attitudes towards sex, and restrictive emotionality subscales $(\alpha=.93)$; (2) self-reliance subscale $(\alpha=$ $.62)$; (3) aggression subscale $(\alpha=.48)$. A major strength of the MRNI is the explicit inclusion of a measure of sexuality (Thompson \& Pleck, 1995). Though the MRNI-A was designed for adolescents, the target population of the current study, and has demonstrated good internal consistency reliability and convergent validity among a sample of American adolescents (Levant, Graef, Smalley, Williams, \& McMillan, 2008), the scale is not utilized in the current study due to its length.

Pleck, Sonenstein, and Ku published the 8-item Male Role Attitudes Scale in 1993. Seven of the eight items were taken from the MRNS and one item concerning sexuality, "Men are always ready for sex," was taken from Snell and colleagues (1986) SAMSS. The items were reworded such that they were made appropriate for 
an adolescent male population. The main strengths of this scale is the strong evidence of construct validity and discriminant validity (in relation to gender attitudes more broadly), as well as the brevity of the scale. However, inversely related to the advantage of the scale's size is the disadvantage of lower reported internal reliability ( $\alpha=.56)$ (Thompson \& Pleck, 1995). Though the MRNS has several strengths that make it appealing for the current study (e.g., brevity and psychometric assessments within adolescent male population), the AMIRS described in the paragraph to follow was selected above the MRNS due to its stronger psychometric property of adequate levels of internal consistency reliability.

In 2005, Chu and colleagues published the 12-item Adolescent Masculinity Ideology in Relationship Scale (AMIRS). Taking into consideration the deconstructionist movement and in attempting to contextualize masculinity, AMIRS measures masculine ideology within the context of boys' interpersonal relationships by assessing the boys' attitudes and beliefs surrounding appropriate behaviors for males in a social environment (Chu, Porche, \& Tolman, 2005). The AMIRS adopts a normative perspective to measure the extent to which hegemonic masculinity is internalized by adolescent boys (Chu et al.). The respondents of the AMIRS indicate their level of agreement to belief statements regarding masculinity using a four-point Likert scale, ranging from (1) disagree a lot, to (4) agree a lot.

Though the authors of the AMIRS theorized the scale to capture several aspects of masculinity ideology, the scale itself was determined to be statistically unidimensional based on an Exploratory Factor Analysis. Additionally, the authors 
have demonstrated reliability $(\alpha=.70)$ of the measure in a combined sample of seventh and eighth graders, and high school boys. Further, the authors have demonstrated validity of the AMIRS in several different ways. First, construct validity was determined by correlating scores on the AMIRS with scores on the MRAS (Pleck et al., 1993) $(r=.53, p<.001)$ and with the three subscales of the MBS: Restrictive Emotionality $(r=.41, p<.001)$, Inhibited Affection $(r=.26, p<$ $.05)$, and Exaggerated Self-Reliance $(r=.31, p<.05)$. Second, discriminant validity was determined by comparing AMIRS to Bem's Sex Role Inventory described in detail in Chapter II. Whereas the AMIRS was significantly correlated with the MRAS (reported above), the Attitudes Towards Women Scale for Adolescents (AWSA; Galambos, Peterson, Richards, \& Gitelson, 1985) $(r=-.55, p<.001)$, and the Rosenberg Self-Esteem Scale (Rosenberg, 1965) $(r=-.32, p<.001)$, and the Bem Sex Roles Inventory was not significantly correlated with any of the measures. Concurrent validity was assessed by examining the relationship between AMIRS and AWSA, Self-Esteem, and Acting Out Index (ADD Health; Resnick et al., 1997), a measure of aggressive or deviant social behaviors $(r=.27, p<.01)$. Finally, predictive validity was assessed by determining the predictive qualities of the AMIRS on self-esteem, controlling for depression $\left(\Delta \mathrm{R}^{2}=.31\right)$, followed by MRAS $\left(\Delta \mathrm{R}^{2}=.11\right)$, and AWSA $\left(\Delta \mathrm{R}^{2}=.03\right)$.

The current study uses the AMIRS as the measure to assess masculinity ideology. As may be understood from the reasoning provided above, compared to alternate measures, the AMIRS has several notable advantages that provide a strong 
justification for use in the current study. First, the AMIRS is one of only a few measures whose aim is to assess masculinity ideology in adolescent populations. Additionally, the AMIRS has demonstrated several good psychometric properties, including acceptable (or nearly acceptable) levels (Nunally, 1978) of internal consistency reliability in samples of middle-school and high-school aged adolescents and several types of validity (Chu et al., 2005). In comparison to the Masculine Role Attitude Scale (MRAS; Pleck, Sonenstein, \& Ku, 1993), which is similar to the AMIRS in that it also assesses masculine ideology within the context of interpersonal relationships, the AMIRS is different because it is less focused on the absolute need for privilege and power in relationships, focusing rather on the need to maintain an appearance of having privilege, consistent with the normative perspective (Thompson \& Pleck, 1995).

Overview of Masculinity Ideology Research

Over the past two decades, several studies have assessed masculinity ideology, diversity of levels of masculinity ideology among diverse samples, and the relationship of masculinity ideology to behavioral or psychological outcomes. Given the masculinity ideology measurement background described above, a brief overview of masculinity ideology research can now be easily understood. In the paragraphs to follow I provide a brief review of the findings from some key studies assessing masculinity ideology in the field. As will be evident in this review, there is a need for a continuation of research that assesses masculinity ideology in various contexts and with diverse groups. I conclude by presenting arguments that have been 
made recently by some of the most prestigious masculinity researchers, I follow with a selected literature review on masculinity ideology in adolescents, ethnically diverse samples, and in prison populations in the chapter to follow.

Recently, Levant and Richmond (2007) reviewed 15 years of research on masculinity ideologies that had used the MRNI (most using the original MRNI) and found masculinity ideology to be related to a number of different variables. For example, endorsement of traditional masculinity ideology has been associated with several demographic variables such as sex (being male), race and ethnicity (African Americans greater than Latinos, greater than Whites), geographic location (Southern United States greater than Northern states), and nationality (Chinese and Russians reporting greater masculinity ideologies than Americans). Moreover, endorsement of traditional masculinity ideology has been associated with generational differences (sons greater endorsement than fathers), less social support in gay men who endorse greater levels, fear of intimacy, lower relationship satisfaction in heterosexual couples, negative attitudes about racial diversity and women's equality, negative attitudes toward help-seeking, predictive and retrospective reports of acquaintance sexual aggression, less forgiveness and higher degrees of alexithymia. On the other hand, lower levels of endorsed traditional masculinity ideology were related to higher scores on measures assessing opposition to racism and greater openness to diversity. Levant and Richmond note some limitations in their review, most notably the overuse of college student samples and review of doctoral dissertation research. 
In a similar vein, O’Neil (2008) reviewed 25 years of research on masculinity ideologies that had used the Gender Role Conflict Scale (GRCS). Like Levant and Richmond, O'Neil reviewed several studies that demonstrated a relationship between masculinity ideology (using the GRCS) and other variables. For example, O’Neil reported that the majority of studies that assessed masculinity ideology in relation to depression, found a significant and positive correlation between the two such that higher levels of adherence to traditional masculine norms was associated with higher levels of depression. Similarly, research was reviewed that illustrated a positive and significant relationship between traditional masculinity ideology and three measures of psychological distress; (1) anxiety; (2) stress; and (3) poor psychological wellbeing. Additionally, O’Neil reviewed research that has demonstrated traditional masculinity ideology to be negatively related to self-esteem and positively related to alexithymia.

In concluding their review on masculinity ideology research, Levant and Richmond (2007) call for a continued investigation between traditional masculinity ideology and social contexts, individual differences, and relational heath variables. The authors call for a greater attention to understanding how multiple dimensions of diversity interact with each other and with masculinity ideologies, as well as the development of masculinity ideology and how it changes over the life span. Finally, the authors call for a continuation of research assessing the relationship of masculinity ideology with problematic individual and relational outcomes.

\section{Conclusion}


Over the past few decades, research on masculinity ideology has multiplied, as have the instruments created to assess the construct. Though each instrument used to assess masculinity ideology has strengths as noted above, the AMIRS is most useful for the current study because it has demonstrated reliability and validity within an adolescent population and the length of the scale is suitable for the purposes of this study. Our understanding of the correlates and outcomes related to differing levels of masculinity ideology has grown over the years. Taken together, a general review of the literature suggests that high levels of adherence to traditional masculinity are associated with negative behavioral, health, and psychological outcomes. In the chapter to follow, Chapter IV, I provide a more detailed review of the literature regarding masculinity ideology within the specific contexts of the current study. In particular, I review literature on adolescent masculinities, race/ethnicity and masculinities and, finally, prison masculinities. 
CHAPTER IV

\section{Contexualizing Masculinity}

The context of the current study, as was described in chapter I, includes a focus on a population of incarcerated adolescents in four different youth detention centers in the state of Ohio. The majority (approximately 60\%) of the youth in the current study, self-identified as African American, followed by those who identified as White, Latino, Native American, Asian, Other, or "Multiple" responding to a combination of race/ethnic categories. For this reason, the review of literature to follow will focus briefly on research conducted on adolescent masculinity ideology in general, then move on to review research that has assessed the influence of race/ethnic identity on masculinity ideology development. The context section of this chapter will include three subsections: (1) Adolescent Masculinities; (2) Race/Ethnicity \& Masculinity; and (3) Prison Masculinities. In each subsection, I provide an overview of the general context (age, race/ethnicity, prison populations), including providing a definition that is relevant to this study. Following this general overview, I review masculinity ideology literature and research that has been conducted within this specific context and population. I conclude this chapter with a summary of what we know about masculinity ideology within the contexts of the current study, as well as what remains unknown and how this study proposes to assess these identified gaps in the literature.

Researchers have called for a continued investigation to understand traditional masculinity ideology within unique social contexts (e.g., Levant \& 
Richmond, 2007; Mankowski \& Maton, 2010) and to assess masculinity ideology in relation to problem behavior (e.g., O’Neil \& Lujan, 2009). Though the contemporary deconstructionist movement pushes one to consider the contextual and environmental influences on the individual, as well as the individual influences on the context, masculinity ideology research that pursues this worldview is lagging. As a means of demonstrating current lack of understanding of the relationships between masculinity ideology and the three context related to the current study, being (1) age; (2) race/ethnicity; (3) prison, I present the results of a search of published literature using PsychInfo, as was used in Chapter II.

\section{Adolescent Masculinities}

Because this dissertation proposes to study masculinity ideology among adolescent males, I begin this section by defining adolescence and providing a brief review of the field of adolescent research. To follow, I provide a review of the literature that describes the differences in masculinity ideology across age. Finally, I review the literature that directly assesses masculinity ideology within an adolescent population. To conclude this subsection, I apply this review of the literature to the current study and describe how the current study addressed the gaps in the literature.

Adolescence. Adolescence is characterized as a diverse, transitory period of development, made up of several distinct stages. As such, a single definition of adolescence cannot be established. Rather, developmentalists tend to agree on a common set of definitions of adolescence including a biological, psychological, and sociological definition (Cobb, 1992). In the following three paragraphs, I will 
describe three generally accepted definitions of adolescence according to these three distinctions.

Through the biological lens, adolescence is often characterized by the physical changes that occur as the result of puberty. Though adolescence has often been described by age for convenience, researchers have noted the onset of puberty as a better marker for the beginning of adolescence (e.g., Peterson, 1988). Puberty status refers to the changes that are experienced by the individual as he or she matures and puberty timing characterizes the timing of these changes as compared to a reference group of their same-aged peers (Peterson). In boys, physical changes that occur during this period of time include growth spurt, beginning around the age of 12 and peaking around 14 years old, development of muscles, the growth of facial and pubic hair, onset of ejaculation, and development of sweat glands (Cobb, 1992). For boys, early puberty timing is generally a positive experience because with puberty boys gain muscle and strength (e.g. Peterson). In the current study, puberty status has not been assessed and only a measure of age can be used to demonstrate the developmental stage of the population.

Adolescence as defined through a psychological lens has been characterized as a period of time in which an individual is faced with a series of tasks that enable her or him to create a stable identity that may transcend different roles the individual may have and the different experiences s/he may face. Simply stated, the psychological definition of adolescence is a period of time in which an individual achieves a continuing and stable sense of self (Havighurst, 1972). The tasks in which 
an adolescent faces have been broken down into those faced in early adolescence and those faced in later adolescence. Most notable to this current study, early adolescence is characterized as a time in which an individual achieves a masculine or feminine social role. This means that although adolescence is biologically determined by puberty, in which physical attributes of a sex (or multiple sex's) are determined, psychological development of a gender role also occurs. This task of achieving a gender role is influenced by our cultural standards of being masculine -strong, active, assertive, or feminine -- passive, dependent, weak (Cobb, 1992). In the current study, sex and gender role has not been assessed, but gender (masculinity) ideology will be used to assess the adolescent's adherence to these traditional cultural standards of masculinity.

Finally, through a sociological lens, adolescence has been defined as a period in which an individual is not completely self-sufficient, thus, not fully an adult, while neither being completely dependent, thus, not fully a child (Cobb, 1992). For example, adolescence is a period that is marked in our country by legislation specifying age limits for activities such as voting, drinking alcohol, and driving. Where this breaks down is in the prison system. As noted previously in this dissertation, the population of study is on adolescents in prison. Therefore, it is important to note that the sociological definition of adolescents in prison may be different from those of the general population. Adolescents in prison have less independence and less freedom to be self-sufficient. Therefore, even for those in their late teens or as old as $20-21$ years of age, legislation that allows adolescents of 
a certain age to drive, vote, and drink alcohol are privileges denied their peers in prison. Additionally, incarcerated adolescents are dependent on the prison system for food and shelter, hindering their transition into adulthood to wait until they are released. Thus, in the current study, all participants are considered adolescents even if they are in their late teens or early 20 's, in that their ability to live self-sufficiently is suspended until they are released.

A Brief History of Research on Adolescence. Contemporary researchers have cited Hall (1904) for first recognizing adolescence as an important period in life, one in which he coined the term, "storm and stress" ${ }^{1 "}$ (e.g., Peterson, 1988). Hall described conflict with parents, mood disruptions, and engaging in risky behaviors as the key aspects of adolescence. By mid-century, researchers Piaget (Inhelder \& Piaget, 1958) and Erikson $(1950 ; 1968)$ dominated the field. Piaget described a theory of developmental stages beginning with the sensorimotor stage (birth to 2 years), preoperational stage (2-7 years), concrete operational stage (7-12 years), and the formal operational stage (12 years onward). Erikson postulated eight stages of development: Trust vs. mistrust (infant), autonomy vs. shame and doubt (toddler), initiative vs. guilt (preschooler), industry vs. inferiority (school-age child), identity vs. role-confusion (adolescent), intimacy vs. isolation (young adult), generativity vs. stagnation (middle-age adult), and integrity vs. despair (older adult). During this time, the meta-theory or worldview of organismic (e.g., Altman \& Rogoff, 1987) was dominant. By the 1980's, researchers began to argue for a more ecological worldview (e.g., ecological model, Bronfenbrenner, 1979; transactional worldview, 
Altman \& Rogoff), such that researchers took into account the environment in which the adolescent resides (Peterson, 1988). In general, research has supported Erikson's model of development, though the timetable in which the stages are said to occur are inaccurate. For example, research has demonstrated that early adolescence is characterized less in terms of social comparisons and more in terms of personal beliefs and standards; middle adolescence is described as discrepant, where individuals describe themselves in opposing ways depending on context; and late adolescence is the period in which the majority of the identity work occurs (Steinberg \& Sheffield, 2001).

Since Peterson's (1988) review of adolescent literature, the field of adolescent research has grown immensely (Steinberg \& Sheffield, 2001). This growth has been explained using four broad trends: (1) a greater emphasis on the ecological perspective (e.g., Bronfenbrenner, 1979); (2) methodological improvements in studying the biological components of adolescence (e.g., puberty); (3) a shift in funding to more applied research; and (4) a shift in methodologies that better capture development (e.g., longitudinal studies). Currently, the field of adolescent development is dominated by research on family context, problem behavior, and puberty or the impact of puberty (Steinberg \& Sheffield, 2001). Problem behavior is defined as behavior that is socially regarded as problematic, undesirable, concerning, or that elicits some form of social control response (e.g., approbation, incarceration). Although the study of problem behavior in adolescence dominates the literature, there is a lack of evidence that supports the idea that adolescents are at greater risk of 
engaging in problem behavior than people of other age groups (Steinberg \& Sheffield, 2001). Steinberg and Sheffield (2001) note three problems with problembehavior research: (1) we need to distinguish between occasional experimentation and enduring patterns of dangerous and troubling behavior; (2) to distinguish between problems that have their origins and onset during adolescence and those that have their roots in earlier developmental periods; (3) identifying problems experienced in adolescence that are relatively transitory in nature and are often resolved by the onset of adulthood, with few long-term repercussions.

The study of problem behavior is particularly important to address in this literature review because it has long been associated with masculinity. For example, masculinity ideology has been linked to substance abuse (e.g., Blazina \& Watkins, 2000), abusive behaviors (e.g., Senn, Desmarais, Verberg, \& Wood, 2000), violence and aggression (e.g., Cohn \& Zeichner, 2006), including relationship violence (e.g., Jakupcak, Lisak, \& Roemer, 2002), and hostility toward women (e.g., Rando, Rogers, \& Brittan-Powell, 1998; Senn et al., 2000). In the subsection below, I will describe how masculinity ideology has been assessed in this population and what it has been associated with directly.

Masculinity Ideology in Adolescence. Because adolescence has been psychologically defined as a period in life in which one strives to adhere to a certain gender role and a stable sense of self, understanding masculinity ideology within this developmental stage is important. Moreover, understanding whether and how masculinity ideology is dynamic over time is theoretically important to 
understanding the developmental process of adhering to a gender ideology. Unfortunately, little is known regarding the development of masculinity ideology over time (Abreu , Goodyear, Campos, \& Newcom, 2000). Masculinity ideology, specifically within the adolescent developmental period, has itself only recently come under investigation. In a review of the literature using PsychInfo and the keywords "Masculinity" and "Adolescent Development," only 84 sources were identified. Moreover, after limiting the search for those references that were listed as utilizing a longitudinal methodology, only four sources met this criterion. In the following subsection, I describe what is currently known regarding masculinity ideology in adolescent populations and how it differs from boys and men of other developmental stages. To begin, I describe in detail some of the key studies that assess masculinity ideology within adolescent populations. I conclude this subsection with a brief summary of how masculinity ideology is known to vary with age.

Pleck, Sonenstein, and Ku (1993) were the first to assess whether adherence to traditional masculinity was a predictor of problem behavior in adolescent males. The authors examined the 1988 National Survey of Adolescent Males (NSAM), in which 1,880 males ages $15-18$ years old were selected using stratified sampling to over-represent minority adolescents (e.g., Black and Latino respondents). Participants were interviewed for approximately 75 minutes each with a focus on assessing the relationship between adherence to traditional masculinity ideology and problem behavior. A measure of masculine ideology was created using 8-items adapted from Thompson \& Pleck's (1986) Male Role Norms Scale and a 26-item 
abbreviated version of the Brannon Masculinity Scale, Short Form (Brannon, 1985). The 8-items were selected based on their relevance with an adolescent population and to represent three factorial dimensions -- status (3-items), toughness (2 items), and anti-femininity (2-items). It is important to acknowledge that the reliability of the scale within this particular sample is below adequate $(\alpha=.56)$. Adolescent problem behavior was assessed using two items assessing difficulties with school (i.e., repeated a grade, suspended from school), three items weighing the frequency of alcohol and drug use over the past year, two items assessing general delinquent activity resulting in contact with the police, and three items assessing sexual activity. Masculine ideology was determined to have a significant independent association with seven of the ten items assessing problem behavior. Thus, adolescent males' problem behavior is related to greater endorsement of traditional masculine ideology. Specifically, adherence to traditional masculinity ideology was significantly and independently associated with seven of the ten problem behaviors assessed: (1) school suspension; (2) alcohol use; (3) drug use; (4) arrests; (5) sexual activity; (6) number of heterosexual partners; and (7) perpetration of coercive sex. Additionally, the authors concluded that masculine ideology is a distinct (i.e., independent from masculine gender-related personality traits and attitudes toward women) component of a man's involvement with his gender role. Taken together, the results from this study inform the current study in demonstrating masculinity ideology as a valid construct to assess gender role involvement in adolescent populations. Moreover, as has been demonstrated in adult populations, this study established a relationship 
between adherence to traditional masculinity ideology and problem behavior. The adolescents in the current study may all be considered to engage (or to have previously engaged) in problem behavior because they are all labeled by the judicial system as "felons."

In 1995, Cournoyer and Mahalik addressed the research question regarding the dynamic nature of masculinity ideology by comparing 88 college-aged men $(M$ $=19.81$ years, $S D=1.35)$ with 89 middle-aged men $(M=40.96$ years, $S D=2.83)$. Though the authors report moderate amounts of gender role conflict in both groups, significant differences across the four factors of gender role conflict previously described were reported between the groups. Specifically, middle-aged men experienced less conflict than college-aged men on the factor success, power, and competition, $t(175)=-2.90, p=.002$, and more conflict on the factor conflict between work and family, $t(195)=2.10, p=.023$. The findings from this study suggest that some factors of gender role conflict are experienced differently for men of different age groups. However, because this study was cross-sectional and not longitudinal, it is impossible to control for possible historical confounds and thus it cannot speak to the possible dynamic nature of masculinity ideology. The current study, on the other hand, is longitudinal and investigated the dynamic nature of adherence to traditional masculinity ideology over the course of approximately 20 weeks.

In 2005, Blazina, Pisecco, and O'Neil adapted the Gender Role Conflict Scale (GRCS; O'Neil et al., 1986) to be used with adolescent populations. From completed 
surveys of 464 adolescent males $(M=16.2)$, the researchers assessed the psychometric properties of the adapted scale and noticed overlap with adults on factors assessing restricted affection between men, restricted emotionality, and conflict between work, school, and family. However, whereas the adults had 8-items assessing success, power, and competition, the youths' related factor was categorized as need for success and achievement.

As predicted, the adolescent version of the Gender Role Conflict Scale was correlated with psychological distress (Blazina, Pisecco, \& O'Neil, 2005). Specifically, the factor of restricted emotionality was the most consistent factor correlating with multiple dimensions of psychological distress including family problems $(r=.3)$, emotional distress $(r=.38)$, conduct problems $(r=.22)$, and anger management $(r=.25)$. Interestingly, the factor need for success and achievement was negatively correlated with youths' reports of conduct problems $(r=-.3)$. The authors concluded that this factor might not access conflict, but rather positive aspects of masculine ideology and suggested that it may play a buffering role against conduct disturbing behavior. Similar to Pleck, Sonenstein, and Ku (1993), Blazina, Pisecco, and O’Neil (2005) demonstrate a clear relationship between adherence to traditional masculinity ideology and problem behaviors (e.g., conduct problems and anger management).

During that same year, Watts and Borders (2005) interviewed 11 adolescent public high school males to assess the validity of O'Neil and colleagues (1986) four Gender Role Conflict patterns in young men. The authors concluded that the four 
constructs seem to apply to adolescent males and that the theory of Gender Role Conflict resonated for this population. Specifically, the adolescents supported the theme of restricted affection between men, indicating supposed homophobia as a key rationale for avoiding an expression of affection towards other males. The theme of restricted emotionality was also supported, where several adolescents commented on the inappropriateness of expressing any emotions other than anger and rage. The last two themes, conflict between work or school and family and need for success and achievement, though supported, were less clear. Boys who were more academically motivated expressed more conflict and tended to agree that it was important to feel in charge even though they struggled with a concrete definition of success.

Taken together, the work of Blazina and colleagues (2005) and of Watts and Borders (2005) has provided a greater understanding of how gender role conflict is understood in adolescent populations. Specifically, adolescent boys seem to experience some of the same dimensions of gender role conflict as adults. In particular, restricted emotionality and affection between men are dimensions of gender role conflict that appear to be experienced both in adolescence and adulthood. However, in dimensions regarding success, power, and achievement, and to a lesser extent in conflict between work or school and family, the overlap is more ambiguous. Blazina and colleagues speculate that although the adult factor regarding success and power may be conceptualized as a gender role conflict, the adolescent factor regarding need for success may reflect positive aspects of masculinity ideology. To be clear, the authors acknowledge adolescence striving for success and 
achievement as an aspect of traditional masculinity ideology that may buffer against problem behavior outcomes such as conduct disturbances. Additionally, the factor regarding conflict between work or school and family was not consistently reported among the youth, where young men who were more academically motivated expressed greater conflict than those who were less motivated (Watts \& Borders, 2005). Due to this continued ambiguity regarding some of the dimensions of gender role conflict, and thus masculinity ideology among adolescents as compared to adults, Watts and Borders call for longitudinal studies to assess the trajectory of gender role conflict in males.

In his longitudinal research project, Listening to Boys' Voices Study, beginning in 1996, Pollack has made substantial contributions to our understanding of adolescent masculine development. In the first phase of the Listening to Boys' Voices Study as reported by Pollack (2006b), 150 mostly white and middle-class adolescents participated in a survey and one-on-one interviews about their experiences of being a boy. The research demonstrated a discrepancy between boys' outward expressions of themselves and their subconscious feelings and emotions. Specifically, Pollack notes that underneath the "mask" of masculinity (e.g., bragging, self-confidence) were relational boys who worried about the quality of relationship with family and friends and empathetic boys who were sensitive to the needs and emotions of others. Furthermore, boys expressed confusion about what behaviors are considered masculine today thereby supporting an idea of a double-standard of masculinity, in which boys endorse both egalitarian and traditional masculine norms. 
The research demonstrated that as the boys grew older, their inner conflict about masculinity was exacerbated, leading them to outwardly act self-confident when they often feel lonely and alienated. The results from Pollack's longitudinal study may inform the developmental trajectory of masculinity ideology among adolescent males. An important implication of this study is the reported "mask" of masculinity that was worn by the participants. Pollack's study utilized two methods of inquiry, as noted above: (1) pencil and paper survey; and (2) one-on-one interview. The "masks" were described for the interviewing method, which is no surprise given that the greater the role of the researcher or interviewer in data collection, a data collection methodology known to produce higher inaccuracies in reporting due to social desirability (Fowler, 1995). The current study will relyied on anonymous paper-and-pencil self-reports of adherence to traditional masculinity ideology and open-ended responses regarding masculinity only.

Collectively, research assessing masculinity ideology among adolescent males, and in comparing young men to middle-aged men, has shed some light on the question regarding how masculinity ideology is experienced among young people differentially from adults. Adolescent-focused studies have demonstrated that adherence to traditional masculinity ideologies in adolescence is associated with problem behaviors (Pleck, Sonenstein, \& Ku, 1993), paralleling research with adult males. Additionally, research with adolescent populations has demonstrated that masculinity ideology factors regarding gender role conflict associated with restricted 
emotionality and affection between men tend to hold up well among this population (e.g., Blazina et al., 2005; Watts \& Borders, 2005).

Taken together, the literature described above highlights a need to measure masculinity ideology differently and separately for men at various stages of development. For example, in comparing college-aged men with middle-aged men, some factors of gender role conflict were experienced differently between the groups (Cournoyer \& Mahalik, 1995). Additionally, Pollack has written about boys’ tendency to experience a discrepancy between their inner feelings of masculinity ideology and their outward expressions of masculine norms. In this study, Pollack describes an effect of age, reporting the conflict between a young man's inner ideals of masculinity and outward expression seemed to be exacerbated with age. Again, what can be taken from these studies is that some factors of gender role conflict and masculinity ideology are experienced similarly between young men and middle-aged or adult males. However, other factors, most notably those regarding success and achievement and conflict between work and family, seem to be experienced differentially between the groups. For these reasons, the current study sought out a measure of masculinity ideology that has demonstrated good reliability and validity among a population of adolescents.

Although the studies described above have opened the doors to understanding the potential dynamic nature of masculinity ideology and how it is experienced among adolescent populations differently from adult populations, several researchers continue to acknowledge some major holes in current understanding of adolescent 
masculinity. For example, although research has demonstrated a relationship between boys' restricted gender roles and their psychological and emotional problems, how male gender roles contribute to these specific problems is relatively unknown (O'Neil \& Lujan, 2009). Additionally, although researchers have concluded that gender role conflict is experienced differentially between age-cohorts (e.g., Cournoyer \& Mahalik, 1995), the unique growth trajectories of endorsement of traditional masculinity ideology within an individual remain unclear. The few studies that have examined a relationship between age and adherence to traditional masculinity ideology have reported mixed findings. For example, Levant and colleagues (1992) reported a small but significant negative correlation $(\mathrm{r}=-.22)$ between age and adherence to traditional male role norms among a mostly undergraduate sample. Similarly, Pleck and colleagues (1994) identify a subsample of younger adolescents who adhere to greater levels of traditional masculinity ideology. On the other hand, Abreu and colleagues (2000) report small but positive regression coefficients of age (after controlling for ethnic identity and level of ethnic belonging) predicting two dimensions of masculinity: Status-respect $(\beta=.13)$ and tough image $(\beta=.15)$ among a sample of adolescent males. Perhaps in response to this gap in the literature, Watts and Borders (2005) describe a need for longitudinal studies such that the trajectory of gender role conflict can be more clearly understood. Similarly, Abreu and colleagues conclude their study with a suggestion of a possible non-linear relationship between age and masculinity ideology. One goal 
of the current study was to respond to this identified gap in the literature by assessing both linear and non-linear change in masculinity ideology over time.

Research on adolescent masculinity is limited and has been primarily conducted with college-aged men (e.g., Levant et al., 1992) or white boys of middleclass background (e.g., Pollack, 2006b); or, through using measures of masculinity used with the adult populations without demonstrating validity with adolescents. Even with the more recent additions of Blazina and colleagues (2005) quantitative study and Watts and Borders (2005) qualitative study with public high school adolescent males, generalizability of these findings are still quite low. In fact, Watts and Borders call for additional studies to assess more diverse populations to better understand how gender role conflict is manifested across different racial and ethnic groups. For these reasons, the current study aims to assess differences and similarities of trajectories of masculinity ideology over time in adolescent men across race/ethnic identity and status. In the section to follow I will define ethnicity, as it is understood in the current study, and follow with a description of the literature that assesses masculinity ideology and ethnicity.

\section{Race/Ethnicity \& Masculinities}

Researchers have recently gained interest in variations of gender ideology among individuals of different cultural, racial or ethnic backgrounds. In this section I will review literature that assesses the intersection of masculinity ideology with ethnicity and race. Because ambiguity often exists around definitions of culture, race, and ethnicity, I will begin by defining each of these concepts. I will then briefly 
describe how race/ethnicity is assessed in the current study and provide an operationalized definition for this purpose. To conclude this section, I will review the literature that examines the intersection of masculinity ideology development with race/ethnicity directly.

To be clear, culture, ethnicity, and race can rarely, if ever, be defined in such a way that is agreeable to everyone (e.g., Segall, 1984). However, to the extent to which these concepts are "measured" in research, a clearly stated definition must be made. Therefore, I will briefly define each of these terms before reviewing the literature that assesses them in the context of masculinity ideology research. To begin, Betancourt and Lopez (1993) define culture as a set of shared values, attitudes, and systems of meanings that are learned and transmitted from one generation to another. Race has been defined in terms of biological factors and physical characteristics, such as skin color, hair type or color, and facial features (e.g., Zuckerman, 1990). However, as is obvious in this definition, problems tend to arise due to the lack of flexibility in stating variations existing within a racial group. Moreover, there is widespread disagreement in defining race, especially in defining race operationally for the purposes of psychological research (Phinney, 1996). For this reason, scholars have sometimes used the term "ethnicity" to encompass both race and cultural origin (Phinney). Specifically, ethnicity is often used to refer to groups characterized in terms of nationality, culture, or language. It is important to recognize that the concepts of ethnicity, race, and culture are related and are often used interchangeably (Betancourt \& Lopez). In the current study, the terms "race" 
and "ethnicity" are used together, as "race/ethnicity", to encompass both race and culture.

In psychological research, as is the case with the current study, the impact of race/ethnicity is often examined on the outcome of interest (i.e., masculinity ideology). Phinney (1996) acknowledges three important dimensions of ethnicity that might account for this impact on psychological outcomes of interest: "(a) cultural values, attitudes, and behaviors that distinguish ethnic groups; (b) the subjective sense of ethnic group membership (i.e., ethnic identity) that is held by group members; and (c) the experiences associated with minority status, including powerlessness, discrimination, and prejudice" (p. 919). Though these three aspects are separated in the above description, they are not independent and will be indistinguishable in the current study.

The current study measured one's race/ethnicity with the single statement, "please circle your race/ethnic identity". The response options are "White," "Asian," "Latino," "Native American," "African American," and "Other." Respondents are encouraged to circle all that apply and to write in their unique identity in the space next to the "other" category. Additionally, the current study has access to juvenile justice facility records that also have an indicator of "race," which distinguishes between "Black," "Hispanic," "White," “Other," and "Multiple." As is the case with most psychological research, race/ethnicity in the current study is measured as discrete categories that represent only the broad and generalized groupings of these American adolescents. Clearly, this opperationalization of the terms limits the 
complex multidimensionality that is inherent to these constructs. How I account for fluidity in identity over time is an additional limitation with the race/ethnic identity categories in the current study. It is anticipated that some youth may change their response to this question from one measurement occasion to another. I describe a process of accounting for this in the analytical model in the methods chapter to follow. Additionally, I describe the responses that were not captured by the broad categories in the model and describe the patterns of change in identity on a case-bycase basis.

In addition to the limitations of categorizing race/ethnicity noted above, it is important to note that within-group (within-category) membership is heterogeneous and variable. Thus, the interpretation of results from a model in which race/ethnic identity alone predicts a psychological outcome (i.e., masculinity ideology) is limited. Specifically, one aspect of heterogeneity within a race/ethnic group, is the variation in strength of identification with that group (Phinney, 1996). To account for this aspect of within-group variation, the current study also examined degree of ethnic pride. At this juncture, it is important to acknowledge differences in identity salience across groups. As is the case with the male sex, members of the dominant racial/ethnic group (e.g., White) may place less importance on that part of their identity (Phinney). For this reason, ethnic pride was be assessed uniquely for each race/ethnic category. With this brief review of definitions of culture, ethnicity, and race, I now review the literature that assesses the intersection of masculinity ideology and race/ethnicity. 
The term "masculinity ideology" was proposed by Thompson and Pleck (1995) to acknowledge the socially, culturally, and contextually constructed nature of masculinity. In the vein that there exist multiple masculinities, theorists propose that people of different backgrounds experience differing types or levels of adherence to traditional masculine norms. Though theorists tend to propose multiple masculinities, specifically masculinities that differ across race/ethnic groups, a simple review of published literature suggests that research assessing masculinity ideology and ethnic identity is limited. A review of PsychInfo using the keywords "Masculinity" and "Ethnic Identity” revealed only 45 sources. In the paragraphs to follow, I first introduce the concepts of cool pose to describe masculine African American men and machismo to describe masculine Latino men. Because the masculinity ideology theory (i.e., Brannon, 1976) described in previous chapters was based, for the most part, on White men, it is not described again in the current section. To follow, I review some of the key studies that have assessed masculinity ideology across racially/ethnically diverse groups. As has been accomplished throughout this document, I present the studies in the order they were published in the literature.

Racism and discrimination must be considered in order to fully understand how masculinity ideology may be different based on the ethnicity of a man. Thus, in order to understand masculinity ideology among African American men, we must also consider historical influences among this particular ethnic group. Black men learned from the start that the traditional ideals of the American man did not provide 
the same reward as it did for White men (e.g., Majors \& Billson, 1992). In fact, before the onset of the Civil Rights Movement of the 1960's, Black men were not even considered "men," as they were generally referred to as "boys" (Kimmel, 2007). The development of a masculine gender identity for African American men has taken on a different trajectory than for European American men, because Black men were not given access to the gains of the same traditionally masculine ideals that were afforded to their White counterparts (Kimmel). As a result, African American men created their own alternative forms of masculinity, such as the cool pose described in detail by Majors and Billson.

Cool pose is a manner in which African American males present themselves socially in order to establish a masculine identity and survive psychologically (Majors \& Billson, 1992). The cool pose represents a script of masculinity for African American men that includes a set of expressions or messages that meet the bill of the cool pose. For example, the cool pose is expressed through a physical posture and presentation that illustrate the underlying message or ideal of pride, strength, and control (Majors \& Billson). In this way, cool pose is a restricted form of masculinity that is emotionless and brave, similar to the traditional ideals presented in previous chapters.

The term Latino is used to describe men from Spanish-speaking countries in the Americas. Thus, the term used to describe one ethnic group consists of a variety of different races and countries. Typically speaking, Latino cultures place great importance on immediate and extended family (familismo). The family arrangement 
of this culture is typically hierarchical, where the fathers are ascendant and are expected to be authoritative and dominant. The mothers, on the other hand, are expected to be submissive. With regard to masculinity, a stereotype of this culture centers on machismo, where men are pressured to enact a strong and aggressive form of masculinity. However, researchers and theorists tend to agree that machismo is not a personality trait, but a presentation, like the cool pose, that some men embody as a way to survive in a culture in which they feel powerless (Kimmel, 2007).

Now that I have described two alternate forms of masculinity demonstrated by some men of African American and Latino background, I present a review of the literature on masculinity ideology across ethnicities. To begin, in 1994, Pleck, Sonenstein, and Ku examined differences between African American, Latino, and European adolescent males tendency to endorse traditional masculinity ideology using the Male Role Attitudes Scale. The authors reported greater levels of endorsement of masculinity ideology among several subgroups of participants including: (1) younger adolescents; (2) participants with expectations of completing less education; (3) participants with greater church involvement; and (4) among adolescents who were sexually active. In addition, the authors compared variations in endorsement of traditional masculinity ideology between two different settings: (1) among those living in the South, and (2) among those living in the Midwest or West. The authors found greater endorsement of traditional masculinity ideology among participants from the South compared to participants in the Midwest or the West. Finally, of most notable importance to the current review of the literature, the authors 
report greater endorsement of traditional masculinity ideology among African American adolescents compared to White adolescents. Given these findings, it may be argued that regional location within the United States may be a sub-cultural variation within the cultural group, relative to the study by race or ethnic group (e.g., Levant, Majors, Kelley, 1998). For this reason, the current study also considered regional location in addition to ethnic identity. In the current study, however, youth are all Ohio state residents incarcerated within Ohio and the Midwest region of the United States. Thus, there is little variance in regional location. With that said, youth are incarcerated at four unique locations within the Ohio Department of Youth Services. Thus, the current study examined differences in levels of adherence to traditional masculinity ideology controlling for location within ODYS.

In 1997, Levant and Majors also reported variations in the level of endorsement of masculinity ideology across race and, additionally, across gender. The authors report significant differences between African American and European Americans, where African Americans reported greater adherence to traditional masculinity ideology. Not surprisingly, when averaging across ethnicity, men reported greater adherence than women, and the gender main effect size was greater than that of the race main effect. The difference in effect size can provide some insight into the strength of the differences reported across race. Because African American men reported greater adherence to traditional masculinity ideology as compared to European American men, the current study sought to examine whether these findings could be replicated in a sample of incarcerated adolescents. 
In a study of undergraduate European American men and women from a Northeast multiracial, but predominately White state university and African American men and women from a multiracial, but predominately Black Mid-Atlantic region state university, Levant, Majors, and Kelley (1998) found both gender and racial differences in reports of masculinity ideology using the MRNI. African Americans reported higher levels of traditional masculinity ideology on four of the MRNI subscales (Fear and Hatred of Homosexuals, Self-Reliance, Achievement/Status, and Restrictive Emotionality), as well as the total MRNI scale, as compared to their European American counterparts. These results are less pronounced than those found by Levant and Majors in 1997, in that fewer differences were found. Additionally, men reported greater adherence to traditional masculinity ideology than women, as was also demonstrated by Levant and Majors.

Additionally, Levant, Majors, and Kelley (1998) found a moderating effect of geography on the relationship between race and masculinity ideology. Specifically, African American men from the South reported greater adherence to traditional masculinity ideology as compared to European American men from the NortheastMid-Atlantic region or the South. However, African American men from the Northeast-Mid-Atlantic region were indistinguishable from European American men from the Northeast-Mid-Atlantic region or the South. As was the case for Pleck and colleagues (1994), regional location within the United States appears to play an important role in the understanding of the relationship between race and masculinity ideology. Again, the current study's sample includes only resident's from one 
Midwestern state. Thus, this moderating effect cannot be evaluated within the current study, though location within the state was controlled for and explored in the analytical model.

In 2000, Abreu, Goodyear, Campos, and Newcomb used a sample of 378 $($ African American $=76$; European American $=43$; Latino $=259)$ male adolescents (age $=19.29)$ from a west coast community in a study of the intersection of masculinity ideology, ethnic belonging, and ethnic identity. The authors used the Male Role Norms Scale (MRNS; Thompson \& Pleck, 1986) to assess four dimensions of masculinity ideology: (1) Status-Respect; (2) Antifemininity; (3) Tough Image; (4) Violent Toughness. However, given the low reliability alpha's obtained using the fourth dimension, Violent Toughness, the 3-items assessing that factor was dropped from analyses. Ethnic belonging was assessed using the ethnic belonging and attitudes dimension of the Multigroup Ethnic Identity Measure (MEIM; Phinney, 1992). Specifically, ethnic belonging was assessed with the following four items: (1) I feel a strong attachment towards my own ethnic group; (2) I feel strongly about my culture or ethnic group; (3) I feel a lot of pride in my ethnic group and its accomplishments; and (4) I have a strong sense of belonging to my ethnic group. Consistent with some of their predictions, the authors reported ethnic belonging to be the best predictor of traditional male gender role endorsement, followed by ethnicity and age. Though hypothesized to be a predictor, family income was not significantly related to any of the outcome measures assessing traditional male gender role endorsement. The relationship between ethnic belonging and 
masculinity ideology for the entire sample supports the authors' speculation that ethnic and masculine identity develop in tandem in all ethnic groups. Counter to the authors' prediction, African American men had lower adherence to traditional masculinity ideology than European Americans, though, as predicted, Latino's had higher adherence than European Americans. However, in the discussion of these results, the authors note the possible influence of geographic location, as has been demonstrated in the studies highlighted above. For adolescent males surveyed on the west coast, Latinos reported greatest adherence to masculinity ideology, followed by European Americans and, lastly, African Americans (Abreu, Goodyear, Campos, \& Newcomb, 2000).

Interestingly, ethnic belonging was the best unique predictor of masculinity ideology (Abreu, Goodyear, Campos, \& Newcomb, 2000), such that when averaging across ethnic status, greater ethnic belonging was related to greater adherence to traditional masculine norms. These findings support the idea that ethnic identity and masculine identity develop together regardless of the categorical racial/ethnic group membership. Additionally, the results regarding the influence of ethnic identity on masculinity ideology contradict others in the field (e.g., Levant \& Majors, 1997). Specifically, Abreu and colleagues found European American men to have higher ratings of traditional masculinity compared to African American men. The authors speculate that the influence of location (sample of West coast men) may be at least partially responsible for these findings. The current study sought to examine the 
intersection of ethnic status and ethnic belonging and masculinity ideology to help clarify these findings.

In addition to assessing ethnic status and ethnic belonging in relation to masculinity ideology, the authors assessed the relationship between age and masculinity ideology. As it may inform the review of adolescent masculine literature described previously in this chapter, age also played an important role in the analyses and results from Abreu and colleagues. For example, age alone predicted the masculinity ideology dimension of Status-Respect in a way that contradicted previous research assessing the relationship between age and masculinity ideology. For example, whereas Cournoyer \& Mahalik's (1995) found that some male role conflicts decrease with age and Levant and colleagues (1992) report a negative correlation between age and masculinity ideology, such that as a man ages his adherence to traditional masculinity ideology decreases. Similarly, Pleck, Sonenstein, and $\mathrm{Ku}$ (1994) reported greater endorsement of traditional masculinity ideology among younger adolescents as compared to older adolescents. Taken together, these studies suggest a negative relationship between age and adherence to traditional masculinity ideology. On the other hand, the Abreu and colleagues study report a positive relationship between age and adherence to traditional masculinity, such that older men display greater levels of adherence to traditional masculinity ideology compared to younger men. In critically comparing the differences in these studies, Abreu and colleagues report differences in average age, supporting the notion that perhaps the relationship between age and masculinity ideology is 
nonlinear, and thus associated with both increases and decreases in masculinity ideology. Again, these studies are limited by the cross-sectional design of the study. The present study helps inform these reported discrepancies by assessing an individual's level of adherence to traditional masculinity ideology longitudinally.

Finally, in Levant and Richmond's (2007) review of research on masculinity ideologies described above, the authors report several studies that assess demographic differences in endorsement of masculinity ideology. For the most part, the literature reviewed tends to report higher levels of adherence to masculinity ideology among African Americans, followed by Latinos, and finally, Whites or European American. Additionally, differences have been reported depending on geographic or regional location, where greater levels of adherence to traditional masculine norms are reported in the Southern United States as compared to the Northern states. Finally, differences have also been reported across samples divided by nationality. Specifically, Chinese and Russian samples tend to report greater masculinity ideologies than Americans.

Taken together, the review of literature that assessed masculinity ideology across racial/ethnic groups suggest that differences between groups are often present. However, a collective understanding of the effect of racial/ethnic identity on masculinity ideology recognizes that racial/ethnic identity is merely one of a team of factors that may influence one's level of adherence to traditional masculine norms. Specifically, regional location appears to play a moderating role in the relationship between racial/ethnic status and masculinity ideology. Additionally, gender and age 
are important dimensions to consider. Levant and Richmond (2007) conclude their review on masculinity ideology research with a call for a continued investigation between traditional masculinity ideology and social contexts. In particular, the authors call for a greater attention in understanding how multiple dimensions of diversity interact with each other and masculinity ideologies, and the development of masculinity ideology and how it changes over the life-span. To address this call for continued investigation into race/ethnicity's influence on masculinity ideology, the current study assessed race/ethnic identity as a predictor of change in traditional masculinity ideology over time. Additionally, the current study examined the influence of ethnic pride on masculinity ideology development for each racial/ethnic group.

\section{Prison Masculinities}

Before describing the intersection of gender and crime, or more specifically, prison masculinities, I briefly review the context of juvenile justice within the United States. Specifically, I describe the history of the juvenile justice system and the general culture of juvenile justice facilities. Next, I introduce the concepts surrounding juvenile justice rehabilitation and programming, describing the relatively new perspective of strength-based programming to promote positive youth development. As was described in Chapter I, the current dissertation seeks to evaluate the effect of a strength-based program implemented within two of four of the studied juvenile justice facilities within the state of Ohio. The program is described in detail in Chapter VI, a general overview of the topic of strength-based 
programming within juvenile justice facilitates is described in this subsection.

Finally, and most extensively, I review the literature that assesses the intersection of masculinity and prison, with a focus on juvenile justice facilities and masculinity.

Overview of Juvenile Justice in the U.S. The United States Juvenile

Correctional System was created in 1868 in Chicago, Illinois as a means to reform contemporary policies regarding youth offenders during the Progressive Era. Since the late 1800 s, several reforms have altered the system in ways that protected the rights of the youth, while at the same time changing the juvenile justice system so that it was more comparable to the adult system or prison. In our history, there exists some more stringent or more lenient periods of jurisdiction where youth were treated by the adult or juvenile justice system. For example, before the Progressive Era and up to as recent as the early 1900s, youth as young as seven years old were tried and imprisoned as adults. A shift occurred just before the 1960's when adolescents and youth 18 years and younger fell completely under the jurisdiction of the juvenile courts. Again in the late 1900's, paralleled by a steep rise in juvenile crimes, juvenile offenders faced mandatory minimum sentences and the juvenile justice system was made increasingly similar to the adult system. Today, the purpose of the juvenile justice system is to rehabilitate juveniles through providing inmates with the opportunity to attend school, earn their high school diplomas, GED, or college credit. However, what happens in juvenile prisons is somewhat unknown, as juvenile facilities are placed under less scrutiny than adult prisons and adolescent offenders are given less access to the outside world as compared to adult offenders (Bortner \& 
Williams, 1997). With that said, we can look to government census statistics to get some information on juvenile correction facilities and the youth that inhabit them. On average, juvenile offenders that are committed to a public facility are incarcerated for 147 days (Snickmund, Snyder, \& Poe-Yamagata, 1997). In 2006, there were 92,854 juvenile offenders held in residential placement facilities, including private and public, in the United States (Office of Juvenile Justice and Delinquency Prevention, 2008). The study's population is of juvenile offenders in the state of Ohio, thus a closer glimpse into this state's system is important. In the state of Ohio, 39\% of juvenile offenders were being held for person crimes, followed by $25 \%$ who were being held for property crimes, $16 \%$ for technical violations, $10 \%$ for public order, $7 \%$ for drug crimes, and $3 \%$ for status. The age group that is considered a minor or juvenile under law varies from state to state. Ohio, like several other states, has their own juvenile court system. Within the Ohio Department of Youth Services, an offender is considered a juvenile until he is 21 years of age.

Programming in Juvenile Justice Facilities. Traditionally, the United States juvenile justice system has been deficit-based (Barton \& Butts, 2008). In this tradition, rehabilitation and treatment of young offenders has been problem-focused, with the ultimate goal of reducing problem-behavior (Barton \& Butts). Recently, however, researchers and practitioners have begun to question the effectiveness of the deficit-based model of the United States juvenile justice system and a shift in focus has turned to a framework that is strength-based and focused on positive youth development instead (Barton \& Butts). In response, the juvenile justice system has 
begun to implement strength-based treatment programs into their facilities. For example, the locations of the study within the Ohio Department of Youth Services have begun to implement a strength-based program, The Council for Boys and Young Men that is evaluated, in part, in this dissertation and described in detail in Chapter VI.

At the moment, there is not strong evidence that a strength-based positive youth development approach can be effectively implemented within juvenile justice settings (Barton \& Butts, 2008). A recent investigation, nonetheless, offers some encouragement that it is possible to implement strength-based programming within juvenile justice facilities. For example, Barton and Butts conducted an exploratory study of six juvenile justice facilities across the United States. Overall, the researchers associated staff enthusiasm and positive outcomes for youth with the implementation of strength-based practice and positive youth development. These findings lend support to the hypothesis regarding the effectiveness of a strengthbased program within ODYS.

On the other hand, it is also important to recognize that the correctional environment "is not naturally amenable to a strength-based paradigm" (Barton \& Butts, 2008, p. 13). In fact, examination of psychological treatment within juvenile justice has illuminated several competing factors, including some that are related to this battle between traditional deficit-based environment and the new strength-based perspectives (Abrams, Kim, \& Anderson-Nathe, 2005). For example, Abrams and colleagues describe a paradox in which residents are presented mixed messages 
regarding emotional expression where staff were observed responding to displays of emotion punitively, while at the same time encouraging youth to express emotion for personal growth and healing. Thus, as this example highlights, although a strengthbased model is implemented within juvenile justice facilities in the current study, in its recent history, the environment was likely deficit-based and punitive. For these reasons, competing factors may still be at play during the implementation of this program.

Masculinity and Juvenile Justice. Although it is generally known that men commit the vast majority of crime, especially violent crime (e.g., Belknap, 1996), criminology theories tend to ignore this fact and are alarmingly gender-blind (Lutze \& Murphy, 1999; Messerschmidt, 1993). A review of PsychInfo using the keywords "Masculinity" and "Crime" demonstrate a clear linkage that has been made between the two resulting in 187 articles. However, research on the effects of prison on one's masculinity is less understood, where a review of PsychInfo using the keywords "Masculinity" and "Prison" resulted in only 17 articles. Furthermore, when restricting this search to research conducted with adolescents, only one source was located, a dissertation that used ethnography to assess linkages between prison cultures and public high schools, but was conducted only in the public high school setting (Schnyder, 2010). As some criminology researchers (e.g., Liebling, 1999) have acknowledged, research examining prison culture typically use populations of adult males and has virtually ignored other groups, most notably, young inmates. Thus, although a clear connection has been made between masculinity and crime, 
addressing masculinity, in particular, within the prison context is lacking, as is examining the impact of prison environments on adolescent offenders. In the paragraphs to follow, I briefly present a review of literature on prison masculinities and how the literature has informed the study. Next, I review two recent studies that qualitatively examined masculinity ideology among adolescents in juvenile justice corrections facilities. To conclude, I identify the gaps in the literature on adolescent prison masculinities and describe how the current study intends to address these.

In the prison context, adhering to a hegemonic masculine standard may be viewed as a learned response to the criminal inmate culture (Jewkes, 2005; Sabo, Kupers, \& London, 2001). Within a prison setting, inmates struggle to gain respect from their peers, gain status, and access to the resources that are inevitably, due to the context, scarce (Jewkes). Each of these ideals provides the inmate with a better sense of security to battle a climate of fear for personal safety. In adapting to a prison climate norm that evokes fear, inmates must build a reputation that is aggressive, powerful, and that demonstrates physical strength (Jewkes), characteristics that help define traditional masculine norms. However, similar to that of adolescents in Pleck and colleagues longitudinal study described in the previous section, and to the "mask" of masculinity adorned by some African American men, male inmates may merely "perform" a hegemonic masculinity. Thus, asking adolescent males about their adherence to traditional masculine ideologies using methods that are least likely to promote social desirable responses (e.g., paper and pencil surveys), may provide a more accurate account of masculinity ideology among this population. That being 
said, the benefits of qualitative investigations of masculinity ideology among adolescent inmates have provided a strong framework of understanding of this phenomenon. Specifically, two recent studies that have examined masculinity among male adolescents in juvenile justice facilities have greatly influenced the current study. I describe each of these studies in detail in the paragraphs to follow.

In 2008, Abrams, Anderson-Nathe, and Aguilar investigated how young men's gender identities are formed within the context of the juvenile justice system. The researchers used triangulation methods (e.g., Merrick, 1999), in that they utilized three different sources of information to address their research questions. First, observational fieldwork took place for 14-16 months; second, one-on-one interviews were conducted with the youth; and finally, facility record reviews were conducted. The authors reported several emergent themes from the data that addressed their research question from each of the facilities. In the paragraphs to follow I describe the major findings from each facility.

In the first facility, the researchers report three main emergent themes: (1) overarching hegemonic masculine milieu; (2) competitive masculine ideals and behaviors demonstrated by staff; and (3) inconsistent encouragement of residents to experiment with alternative forms of gender expression. An example of how the first theme was demonstrated through the description of the layout of the main recreation room within the facility. The researchers report an overwhelming stereotypically masculine structure of living including a large-screen television, competitive gaming equipment, and a lack of a structured space to sit and communicate (e.g., cluster of 
chairs, etc). The researchers reported staff to be derogatory and critical of the youth, exhibiting powerful and stereotypically masculine behaviors. Finally, the staff typically reinforced the youth's demonstration of traditional masculine behaviors, even when this was inconsistent with the programming the youth received in the facility. In the second facility, researchers reported three main emergent themes: (1) settings role in suppressing residents individuality and expressions of their own masculinities; (2) staff members participation in enforcing a hierarchy of hegemonic masculinity; and (3) the subordinate role of female staff in the facility. Due to gangrelated experiences, incarcerated youth were restricted in what they were allowed to wear and how they expressed themselves. Thus, the first theme was demonstrated in examples such as a group session that took place in the facility where the staff spoke freely, swearing to the group, but not allowing the youth to speak openly back to them. The youth were not allowed to use slang or curse, even when it was modeled by the staff. Hegemonic masculine behaviors were enforced through competitions of power and strength. For example, researchers observed basketball games between "good" players and staff, which excluded youth who were not viewed as competitive players. Finally, researchers observed differential treatment between male and female staff. The residents perceived the female staff to overcompensate for their lack of physical power by enforcing extra rules and limits on the youth. The residents responded negatively to the female staff for setting more stringent rules. Additionally, the researchers observed the male staff reinforcing this differential treatment and thus tolerating sexism within the facility. 
Taken together, observations from the two juvenile correction facilities demonstrate that hegemonic masculinity is both modeled and encouraged within the system. Additionally, adolescents are discouraged from experimenting with alternative expressions of gender identity as they are restricted to behave in very limited ways. Despite the clear linkage that has been made between criminal behavior and adherence to traditional masculinity ideology, healthy masculine alternatives are rarely addressed within the criminal system. Even when they are, the deeply embedded culture within the facility is in paradox to these healthy alternatives by encouraging and modeling hegemonic masculine standards as ideal. The implications of this study are extremely important for assessing masculinity ideology in juvenile correction facilities in the future. Specifically, in the current study, a program that aims to encourage healthy expressions of masculinity, The Council, is evaluated. However, as was the case in the Abram and colleagues (2008) study, even with the introduction of this program, the culture and staff likely provide a paradoxical message of masculinity. Thus, the current study proposes to examined how masculinity ideology changes over the course of several months within the prison in addition to assessing whether the program, The Council, has any effect on this change.

In 2010, Cesaroni and Alvi interviewed 350 adolescent males in juvenile detention facilities in Canada in a series of three separate studies. The goal of the first study was to gain a basic idea of the experiences of adolescent incarcerated males. The second study's goals were to interview first time inmates to examine 
what predicted youths' adjustment in prison in a short-term longitudinal investigation. Finally, the third study examined the adjustment of males in secure detention facilities to compare with findings from study two. For all three studies, voluntary youth participated in a one-on-one interview that lasted approximately one hour. The youth responded to a series of questions about their lives before incarceration in a closed-ended question format. In addition, studies two and three collected open-ended questions regarding the youths' experience in the prison. These data were systematically coded into two key emergent themes: (1) masculinities and the experiences of incarcerated adolescent males; (2) resistance. For the focus of this paper, I discuss the findings from the first key theme below.

Consistent with theories of prison masculinities, Cesaroni and Alvi (2010) identified the adherence to traditional masculine norms, including restricted emotion, hierarchical social structures, and pride in oneself in the vast majority of the narratives collected in the three studies above-mentioned. The authors illustrated this theme with several quotes that included descriptions of male competition, hierarchical structures with a "top dog," usually the biggest guy, and a description of how the prison environment provokes an act or portrayal of a "tough guy." Additionally, the authors reported physical demonstrations of strength as an important medium to experience male bonding. Moreover, demonstrations of strength and power that were expressed with threats, bullying, and aggressive demeanors were identified as ways in which status within the facility was achieved. Peer relationships and conflict were identified as important concerns for the majority 
of the inmates who were interviewed. Specifically, 20-40\% of the inmates reported being victimized during their current sentence and $27-75 \%$ of the interviewees reported a concern for being attacked in their institution. This study provided insight to the lived experiences of incarcerated young male offenders that is currently not well understood. In addition, this study adds to the findings of Abrams and colleagues (2008) by describing how traditional masculine norms are enforced within juvenile justice facilities. Specifically, the youth from this study describe a culture in which young males feel the need to "act" powerful, strong, and unemotional in order to survive and feel safe.

Taken together, the qualitative study of Abrams and colleagues (2008) and Cesaroni and Alvi (2010) provide a strong basis for a continued investigation of masculinity in juvenile prison settings. What is more, findings from these studies have implications for the treatment and rehabilitation of young men in prison. As O’Neil and Lujan (2009) have issued a call for psychoeducational programs to promote healthy masculinities in educational settings, these findings suggest a need to implement these programs in prison settings as well. Finally, although these studies have investigated the formation and demonstration of hegemonic masculinity, they have not directly assessed the predictors of increased hegemonic masculinity. Thus, the current study addressed this gap by investigating changes in adherence to traditional masculine norms, the predictors of changes, and in evaluating the effects of a group-based program to promote healthy masculinity.

\section{Conclusion}


Theorists described masculinity as ever-changing, unfinished qualities that exist in different forms depending on the unique context (e.g., Messerschmidt, 1993). Different contextual influences on masculinity have been examined in various studies. Specifically, researchers have identified age as an important factor in determining an individual's level of adherence to traditional masculinity (e.g., Abreu et al., 2000; Cournoyer \& Mahalik, 1995). Additionally, theorists and researchers have described alternative forms of masculinity taken by men of different ethnicities (e.g., Abreu et al., 2000; Levant \& Richmond, 2007). Finally, the qualitative investigations of adolescent prison environment (e.g., Abrams, Anderson-Nathe, \& Aguilar, 2008; Cesaroni \& Alvi, 2010) identify a norm of heightened hegemonic masculinity. However, the current study is the first of its kind to use the contextual and personal variables of age, race/ethnicity, and location (i.e., four unique prison locations) together to predict changes in adherence to traditional masculinity ideology among adolescents in juvenile justice facilities. Additionally, the current study is the first of its kind to evaluate a program's effectiveness at decreasing adherence to traditional masculinity among adolescent inmates. In the chapter to follow, I describe the research questions and hypotheses that motivate the study. To follow, I describe the methodology, analyses and results, and conclude by describing the implications and limitations of the study. 


\section{CHAPTER V}

\section{Development of Research Questions and Hypotheses}

In the preceding sections, I introduced the topic of masculinity ideology and discussed important predictors of adherence to traditional masculinity. Specifically, I reviewed the literature on masculinity ideology in adolescent populations and discussed how the level of adherence differs from adult populations. Moreover, I presented literature that examinedadherence to traditional masculinity ideology among racially/ethnically diverse populations. I described both the direct and moderating effect of ethnic belonging on the relationship between ethnic identity and masculinity. Finally, I reviewed the literature on masculinity ideology among incarcerated populations, specifically among incarcerated adolescents. In the respective reviews of the literature, I describe each of these individual and contextual constructs (age, race/ethnicity, ethnic belonging, and incarceration) assessed in the dissertation as predictors of adherence to traditional masculinity ideology. In the section to follow, I describe how the current study investigated masculinity ideology over time and how the aforementioned constructs work together to predict change in adherence to traditional masculinity ideology over time. Furthermore, I describe in detail a program aimed at changing expressions and endorsements of masculinity, The Council, which has been implemented in two sites within the study. To situate the program briefly, the United States juvenile justice system has recently begun to take a strength-based perspective focusing on positive youth development within their facilities (e.g., Barton \& Butts, 2008). Amongst this paradigm shift from deficit 
and problem-focused to strengths-based, the site of the study, Ohio Department of Youth Services, began to implement a strength-based program, The Councilfor Boys and Young Men, in some of their facilities. Because The Council's mission is focused on questioning unsafe attitudes about masculinity and encouraging healthy masculine identity development, it is predicted to have an effect on the study outcome, adherence to traditional masculinity. Thus, the current study proposes to investigate the effectiveness of The Council at changing developmental trajectories of masculinity ideology. In the chapter to follow, the mission and structure of The Council will be described in greater detail.

The study is the first of its kind to quantitatively investigate adherence to traditional masculinity ideology in an incarcerated adolescent population over time. The study is the first to assess predictors of change in adherence to traditional masculinity ideology in addition to assessing the effectiveness of The Council at influencing this change. These data provide a more detailed understanding of masculinity ideology among incarcerated adolescents. To help inform and enhance understanding of the quantitative evaluation of The Council's effect on changes of adherence to traditional masculinity ideology, a qualitative examination into the open-ended responses including the question, "What have you learned about being male?", have also been assessed.

\section{The Study}

The purpose of this study was to investigate change over time in adherence to traditional masculinity ideology in a sample of incarcerated young men. 
Additionally, this dissertation examined contextual predictors of change in level of adherence to traditional masculinity ideology. Finally, the dissertation examined the effects of a strength-based program, The Council, at successfully decreasing levels of adherence to traditional masculinity ideology. Hypotheses and Research Questions

As discussed in previous chapters, research suggests that adherence to traditional masculinity ideology is a function of age (e.g., Cournoyer \& Mahalik, 1995). Furthermore, researchers tend to describe the prison climate as one that promotes hegemonic masculine norms (e.g., Abrams, Anderson-Nathe, \& Aguilar, 2008). Taken together, the review of literature on the effect of age (e.g., adolescence) and prison on masculinity, would suggest that adherence to traditional masculinity would increase for incarcerated youth over time. Thus, it is reasonable to assume that change in adherence to masculinity ideology over time is a function of both age and time in prison, as demonstrated by the theoretical model in Figure 1. Furthermore, researchers have identified differing levels of adherence to traditional masculinity ideology among men of different race/ethnic backgrounds (e.g., Abreu et al., 2000; Levant \& Majors, 2007; Levant, Majors, \& Kelley, 1998; Pleck, Sonenstein, \& Ku, 1994). For that reason, the race/ethnic identities most prevalent among the current study's sample have been assessed uniquely in their prediction of change in adherence to traditional masculinity. On the basis of these predictions, I investigated the following hypotheses and research questions. 
Research Question 1: What are the contextual predictors (i.e., program, prison) and individual predictors (i.e., age, racelethnic identity, and ethnic pride) of masculinity ideology development?

\section{Hypothesis 1. Program Effect}

Hla. Program experimental effect: Change in adherence to traditional masculinity ideology will be negative for youth in the experimental group who have participated in The Council, but not for youth in the control group.

The Council program goals include challenging assumptions about traditional masculinity and encouraging healthy masculine identity development. Because masculine identity development is a major focus of the program, it is expected that youth participating in The Council (experimental group) will have different trajectories of change over time in adherence to traditional masculinities than those not participating in The Council (control group). In particular, The Council questions unsafe attitudes regarding masculinity, many of which are foundational components of traditional masculinity ideology as described in Chapter III. Therefore, it has been hypothesized that trajectories of change in adherence to traditional masculinity ideology will decrease for youth in the experimental group as assumptions regarding traditional masculinity ideology are challenged, whereas the youth in the control group will remain relatively stable over time. Because the research design begins with a baseline measure of masculinity ideology before the introduction of the program, change in both a linear and nonlinear pattern were assessed. 
In addition to examining a global program effect, age of the participant and the number of days in prison were assessed as moderators of the relationship between participation in the program and change in masculinity ideology.

H1b. Program dosage effect [Experimental group only]: Negative linear trajectories of change in adherence to traditional masculinity ideology will be strongest for youth with the greatest attendance in The Council and weaker for those with less attendance.

In reference to The Council goals stated above, the amount of participation in the program for youth in the experimental group was expected to influence the trajectory of change in adherence to traditional masculinity ideology over time. To assess this hypothesis, only youth participating in The Council were included in the analysis of the model. Specifically, it was hypothesized that trajectories of negative change in adherence to traditional masculinity ideology will be greatest for youth with high levels of participation in the program and weakest for those with low levels of participation. Participation is measured by the number of hours a youth has attended The Council over the course of each 10-week curriculum.

\section{Hypothesis 2. Age Effect}

H2a. Age will positively predict adherence to masculinity ideology at baseline.

H2b. Age will be a negative predictor of linear change in adherence to traditional masculinity ideology. 
According to existing research, some factors and outcomes of masculinity research are experienced differently for young men compared to adults (e.g., Blazina et al., 2005; Cournoyer \& Mahalik, 1995), whereas some factors of masculinity ideology and associated outcomes parallel masculinity research conducted with adult populations (e.g., Blazina et al., 2005; Pleck, Sonenstein, \& Ku, 1993; Watts \& Borders, 2005). Empirical literature assessing age-related differences in levels of endorsement of traditional masculinity ideology is limited, (Abreu et al., 2000) and what exists does not present a clear picture of the developmental trajectory of masculinity ideology. For example, some authors reported a negative relationship between age and adherence to traditional male role norms (e.g., Levant et al., 1992; Pleck et al., 1994), whereas, others (e.g., Abreu et al., 2000; Pollack, 2006b) report a positive relationship. Given the study's demographic similarity to Abreu and colleagues participants, the current study anticipated a positive relationship between adherence to traditional masculinity and age. Specifically, older adolescents were expected to have greatest level of adherence at baseline and were expected to be more stable over time. On the other hand, younger adolescents were expected to begin with lower level of adherence, but have greatest amounts of increase over time. Thus, the hypothesized predictor of age on initial levels of adherence to traditional masculinity ideology was expected to be positive, but the moderating effect negative.

\section{Hypothesis 3: Race/Ethnicity Effect}

H3a. Race/Ethnic identity will predict adherence to traditional masculinity ideology at baseline. 
H3b. Race/Ethnic identity will predict masculinity ideology development (neutral hypothesis).

According to the review of literature that assessed masculinity ideology across ethnic groups described in the previous chapter, differences in level of adherence to traditional masculinity ideology are often present. However, the ethnic group with greatest adherence or least adherence to traditional masculinity ideology was not consistent across the studies. For example, Abreu, Goodyear, Campos, and Newcomb (2000) reported Latino adolescents to have the greatest level of adherence to traditional masculinity ideology, followed by European Americans and, lastly, African Americans. On the other hand, Levant and Majors (1997) and Levant, Majors, and Kelly (1998) reported African Americans to have greater levels of adherence to traditional masculinity ideologies as compared to European Americans. Additionally, Levant and Richmond's (2007) review of the literature summarizes the literature by describing African Americans as the ethnic group with the tendency to have the greatest level of adherence to traditionally masculinity, followed by Latino men, and finally, European Americans (White men). Thus, the current study assessed each ethnic identity (African American, Latino, White) separately as a unique predictor of change in adherence to traditional masculinity ideology. It was hypothesized that African American adolescents will have greater initial levels of adherence to traditional masculinity ideology as compared to Latino and White adolescents. Additionally, the developmental patterning for each of these groups will be explored. 


\section{Hypothesis 4: Ethnic Pride Effect}

H4a. Ethnic pride will positively predict adherence to masculinity ideology at baseline.

H4b. The relationship between ethnic identity and adherence to masculinity ideology over time will be moderated by ethnic pride.

According to the review of literature on ethnic masculinities, level of ethnic belonging contributes as a predictor of level of adherence to masculinity ideology (e.g., Abreu et al., 2000). Though the current study did not assess ethnic belonging exactly, a single-item assessing one's ethnic pride was used to determine whether ethnic pride uniquely contributed to a prediction of adherence to masculinity ideology and whether the relationship between ethnic status and change in adherence over time was moderated by the participants' level of ethnic pride. It was hypothesized that individuals with the greatest levels of ethnic pride will have the strongest relationship between ethnic identity and changes in traditional masculinity ideology over time. On the other hand, individuals with low levels of ethnic pride will have weak relationships between ethnic identity and change in traditional masculinity ideology over time.

\section{Hypothesis 5: Prison Effect}

H5a. Days in Prison will positively predict initial levels of masculinity ideology.

H5b. Days in Prison will negatively predict change in masculinity ideology. 
Adherence to hegemonic masculine norms is expected to be high within the prison context. However, the effect of prison context is expected to be greatest for those new to the prison. It is anticipated that as an adolescent tenures within the prison, the effect of the hegemonic masculine climate (e.g., Abrams et al., 2008; Cesaroni \& Alvi, 2010; Jewkes, 2005; Sabo, Kupers, \& London, 2001) is expected to be less. Thus, the number of days in prison was expected to negatively predict linear growth in levels of adherence to traditional masculine norms. Specifically, youth who have resided within prison the longest were expected to have high and stable levels of adherence to traditional masculinity ideology, whereas those new to prison were expected to have greatest linear increase.

Research Question 2. What do youth who participate in The Council learn about being male?

Given the noted limitations of studies that employ only quantitative or qualitative methodologies (e.g., Morgan, 1998), this dissertation utilized both in a specified qualitative follow-up sequence design. The purpose of this qualitative follow-up design was to evaluate and interpret results of a principally quantitative study (Morgan). Specifically, the qualitative component was used to provide insight and an enhanced understanding of the quantitative findings or interpretations for poorly understood results. The dissertation assessed responses to an open-ended question regarding masculinity completed by youth in the experimental group at the second and third survey measurement occasions. 


\section{CHAPTER VI}

\section{Methods}

\section{Study Context and Overview}

This study is part of a larger evaluative research project conducted by Dr.

Eric Mankowski and colleagues at Portland State University. I served as project manager and was involved in all aspects of the project. The larger project evaluated the effectiveness of The Council among youth in four different facilities within the Ohio Department of Youth Services (ODYS), the juvenile corrections system for the state of Ohio. Data collection for the evaluation study took place over the course of one year beginning in June, 2009 and ending in May, 2010. Dr. Mankowski and his research team developed a collaborative research partnership with the director of The Council, Beth Hossfeld, in January, 2008 (see Memorandum of Understanding, Appendix A). During the year and a half time period before data collection began within the ODYS facilities, a pilot study was conducted within educational (e.g., school-based and after-school programs) and detention settings across the United States. After concluding the Pilot Study, aspects of the design and survey were adapted based on the quantitative results, as well as from focus groups and feedback from youth participants and facilitators.

Beginning in January, 2009, the Social Services Administrator for ODYS, Laura Dolan, contacted Beth Hossfeld from The Council to request that The Council be implemented in her facilities, which provided access to a setting and participants in which the second phase of data collection could take place. During the month of 
May, 2009, Dr. Mankowski and I led a video-conference training for ODYS staff at the experimental facilities to describe the extent of the study and the protocol for administering the surveys. Dr. Mankowski led a second video-conference training for ODYS staff at the control facilities. In addition, in June, 2009, Dr. Mankowski traveled to a facility within ODYS to further train the staff on survey administration and to oversee the administration of the pre-surveys, which served as the baseline measures for Ohio River Valley and Circleville. A week after completing the baseline measure, Ohio River Valley served as the experimental group such that youth within this facility participated in The Council. What this entailed was a weekly two-hour session of The Council in small groups facilitated by ODYS staff social workers. Prior to facilitating The Council, staff completed a two-day training with Beth Hossfeld and a colleague in which the theories and goals of The Council were described and the structure of The Council groups were illustrated.

During the ten weeks after the baseline measure was completed, Circleville served as the control group and did not participate in The Council. On the twelfth week, youth at both facilities completed the post-surveys. The post-surveys are identical to the pre-surveys with the exception of a Satisfaction Scale including both closed and open-ended questions regarding the participation and experience in The Council. Thus, the Satisfaction Scale was only added to the surveys completed at the experimental location, Ohio River Valley. At this juncture, the research team recognized that the number of participants who completed both the pre- and postsurveys was lower than expected. Through communication with the stakeholders of 
the program, including Beth Hossfeld and Laura Dolan, it was decided that two additional facilities within the ODYS system would join the study as an attempt to increase the sample size in both experimental and control groups. Thus, four locations completed the third survey, which again was identical to the Pre- and PostSurvey with the addition of the Satisfaction Survey for the experimental site. The week following the third measurement, Circleville began to implement The Council and joined the experimental group. The new locations, Indian River and Cuyahoga Hills served as the control sites. This cycle of survey administration, 10-weeks of The Council or pre-existing program, followed by survey administration, continued for five total surveys for Ohio River Valley and Circleville, and three survey administrations for Indian River and Cuyahoga Hills. This design is outlined in a visual format in the first table of Table 1.

\section{The Council for Boys and Young Men (The Council)}

The Council Purpose. The Council is a structured support group for boys' age 9-18 years that follows a strength-based approach to promote healthy masculinity (Hossfeld, et al., 2008). The Council is based on Relational-Cultural Theory (Miller, 1991) and Resiliency principles (Bernard, 2004), incorporating theories of masculine identity formation rooted in cross-cultural traditions (Hossfeld, et al.). The Council recognizes boys' strengths and capacities, challenges stereotypes, questions unsafe attitudes of traditional masculinity, and encourages solidarity through personal and collective responsibility (Hossfeld, et al.). It aims to promote boys' natural strengths and to increase their options about being male in today's world. Specifically, The 
Council challenges myths about how to be a 'real boy' or 'real man', increases boys' emotional, social, and cultural literacy by promoting valuable relationships with peers and adult facilitators through activities, dialogue, and self-expression (Hossfeld, et al.). The model intends to respond to boys' increased rates of violent crime, bullying, substance abuse, and risky sexual behaviors detailed in previous chapters of the dissertation. The Council works to enhance boys' skills and options for ways to respond to social, emotional, cultural and economic conditions that may impact their lives (Hossfeld, et al.). Although several programs have been established in many youth-serving organizations aimed to specifically support boys, it is unclear to what extent these programs are effective as a gender-specific model to support pre-teen to adolescent boys' development.

The Council Structure. The Council support groups are designed to meet in a group of six to ten boys of similar age and development with one or two facilitators, lasting for one and a half to two hours each week for a series of ten weeks. The groups utilized one or more of the three distinct The Council curriculum guides -Standing Together: A Journey into Respect (for Ages 9 - 14), Growing Healthy, Growing Strong (for Ages 9 - 14), and Living a Legacy: A The Council Rite of Passage (for Ages 14 - 18). The curricula differ only in their respective 10-week themes and activities (see Table 2).

The general format is designed to be the same for each of the three curricula. Each of the group sessions are expected to proceed in order with the following: An opening ritual, theme introduction, warm-up activities, a "council" type check-in 
opportunity, experiential activities that address gender relevant topics, a reflection and group dialogue component, and a closing ritual. The opening ritual is intended to mark the beginning of the council process and invite the boys into council time while setting a strong positive tone. For example, each boy may ring a bell before taking his place in The Council or engage in a special handshake with a pledge before joining The Council. The facilitator then introduces the group to the chosen theme of the week, including a short description and synopsis of what is planned for the meeting. The warm-up activity is a brief physical activity that follows the introduction of the weekly theme. The warm-up provides the boys with an opportunity to connect and interact physically to build a sense of teamwork. Following the activity, the check-in is a time designed for the boys to express whatever they wish or to say something about the theme for the week.

The Council Content. At this point the purposeful activity (see Table 2) is introduced and implemented as the main component of the group session. The weekly purposeful activities are intended to engage the boys' awareness and skills building potential in a safe and protected environment without the danger of losing connection with others. For example, the theme for week nine in the Growing Healthy, Growing Strong curricula for 9-14 year olds is "Male \& Female: Roles and Expectations". During this activity the boys are asked to brainstorm together to generate a list of qualities that are respectively male and female and list them on the board. In this activity the boys are encouraged to think about their gender and their bodies, minds, roles, relationships, and the ways they express themselves. Following 
the purposeful activity time, The Council is asked to reconvene to allow time for personal reflection. For example, following the activity described above, the boys are asked to reflect on the differences and similarities between men and women, differences in power between men and women, friendships between and within genders, qualities they most like about males and females, and the qualities that the boys feel are most important to grow into a happy and successful man. Throughout this activity, the facilitators carefully encourage the boys to share responses and feelings, interpret themes, explore commonalities, and make the connection between the theme and their experiences in the real world. The group ends with a closing ritual that brings closure to the experience and sends the members out safely with a positive tone of gratitude, and respect. The closing ritual is much like the opening ritual and is designed to unite the council for a final moment to bring awareness to the community spirit. The same closing ritual is used after each group session.

Ohio Department of Youth Services. The Ohio Department of Youth Services is statutorily mandated to imprison youth felony offenders, ages 10-20 years old. To be clear, a felony is a type of categorization of a serious crime including aggravated assault, arson, burglary, illegal drug use or sales, grand theft, robbery, murder, rape, and vandalism to federal property. There are five facilities within ODYS, four of which were partners in the current study. The four locations differ in some ways, and thus a brief description of each is provided below in alphabetical order. In addition to describing the location and other unique qualities about each location, I also provide a brief statement about the role each location plays in the current study. 
Circleville is located in the city of Circleville, situated approximately 30 miles south of Columbus in the center of the state of Ohio. Circleville serves the general population of youth who are convicted felons. In the present study, youth at Circleville participated in the control group for the first 20-weeks, then began The Council, joining the experimental group after the third survey measurement and continued for the last 20 -weeks of the study.

Cuyahoga Hills $(\mathrm{CH})$ is located in Highland Hills, a town southeast of Cleveland in the northern part of the state near Lake Erie. Cuyahoga Hills serves the general population of youth, similar to those located at Circleville. In the present study, youth at $\mathrm{CH}$ participated in the control group for the last three measurements.

Indian River (IR) is located in Massilon, a town 60 miles south of Cleveland. Indian River also serves the general population of convicted felons, similar to those at Circleville and $\mathrm{CH}$. In the present study, youth at IR participated in the control group for the last three measurements.

Ohio River Valley $(O R V)$ is located near Franklin Furnace, which is a city at the southernmost portion of the state. Ohio River Valley serves a diverse population of young men ranging from those considered in the general population to low, moderate, and high-risk sex offenders. This population also includes those with low to moderate mental health needs. In the present study, youth at ORV participated in the experimental group for all five measurements, receiving The Council curriculum for approximately 40-weeks total.

\section{Participants}


The participants in the current study are male, ages 12 to 20 who were incarcerated in one of four facilities within the Ohio Department of Youth Services between June 2009 and May 2010. There were approximately 1,077 youth detained at ODYS at any given time during the study ${ }^{2}$. Throughout the duration of the possible five measurements, 1,447 different youth completed at least one survey. This number is greater than 1,077 based on the revolving-door atmosphere of ODYS, in which youth are detained and released on a regular occasion. At any given survey occasion, approximately 710 youth agreed to participate $(66 \%$ of the estimated possible 1,077). An estimate of the number of youth participating in the study was taken by averaging across the first three measurement occasions.

Of the approximately 1,447 youth who completed at least one survey at one of the measurement occasions, 199 youth were excluded from the dissertation analyses because they had not completed a survey during the designated three measurement occasions and/or because they had moved from one location to another within ODYS during the course of the study. The remaining 1,248 youth will be included in the analyses of this dissertation.

Included Study Participants. The demographic distribution of the dissertation study participants' age and race/ethnic identity is presented in detail in Table 3 . The participants' age ranged from 12 to $20(M=16.37$; $S D=7.45)$ at baseline. The majority of the study's participants identified as African American $(n=810 ; 65 \%)$, followed by White $(n=310 ; 25 \%)$, and Latino $(n=29 ; 2 \%)$. The remaining youth identified as Asian, Native American, multiple ethnicities, other, or did not respond 
to the item $(n=99 ; 8 \%)$. Of the 1,248 youth included in the dissertation analyses, $1,072(86 \%)$ have data from both paper-and-pencil self-report survey(s) and ODYS facility data records from at least one time-point.

Youth in the study were distributed across the four ODYS facilities. The majority $(n=409,33 \%)$ of the youth were from Cuyahoga Hills, followed by Ohio River Valley $(\mathrm{n}=341,27 \%)$, Indian River $(\mathrm{n}=341,27 \%)$, and Circleville $(\mathrm{n}=157$, $13 \%)$. The patterning of responses to survey measurement occasions varied across individuals and locations (see Table 4). Only 190 (15\%) of participating youth completed all three self-report surveys. Similarly, data regarding attendance in The Council were also missing at high rates (47\%-65\% missing). From those with attendance data, distributions are detailed in Table 5, including youth with zero hours of attendance recorded. Average participation in The Council for youth at Ohio River Valley was 13.15 hours $(S D=7.25)$ for the first 10 -weeks and 12.52 hours $(S D=$ 5.79) for the second 10-weeks. Average participation in The Council for youth at Circleville was 10.5 hours $(S D=7.66)$ for the first 10 -weeks and 11.65 hours $(S D=$ 7.17) for the second 10-weeks. Taken together, youth attended approximately six of the ten one-hour Council group weekly sessions.

\section{Procedure}

Social workers at ODYS first introduced the potential research participants to the study during one of their regularly scheduled one-on-one meetings before the youth attended the group in which the first survey administration was to take place. The social workers were trained and instructed to follow a script (see Appendix B) in 
which the study was explained to the youth. An informational sheet and two copies of the consent form were provided. Youth made a decision on their own as to whether or not they wanted to participate and attended a group session with a completed consent form if they chose to participate. The second consent form was provided for them to keep for their own records. At the first group session, the facilitator of The Council or the control group followed the Pre-Test Instruction, again providing the youth with a description of the study, explaining the voluntary nature of their participation and asking for questions from the youth. The baseline survey for the experimental group was administered in a group setting before the start of The Council. Youth returned their signed consent forms to the facilitator. The facilitator was then instructed to follow the script provided on the Pre-Test Instructions and hand out a copy of the survey to each youth. Due to the low literacy rates at the ODYS, the facilitator read each survey item slowly to the youth and read aloud each response option in order, so that those who needed assistance could follow along. When the surveys were completed, the youth were asked to insert their completed survey in a manila envelope, which was sealed after the last survey was returned. After surveys from all groups were completed and stored in sealed manila envelopes, the envelopes were placed in a large box by facilitators and shipped to Portland State University. This procedure was followed for all youth as they entered the study. After consent was obtained, survey administration sessions followed a similar structure to the one described above. Facilitators were asked to follow the Post-Test Instructions for all survey administration sessions after the first 
administration. The facilitators provided the youth with a candy bar or art supplies after completing each survey.

Design

The current study utilized a longitudinal, non-randomized experimental research design. In Table 6a, the complete longitudinal design is displayed followed by a table (Table 6b) describing the data assessed in the current study. The longitudinal design is implemented by surveying participants three to five different times (occasion). The occasion of data collection occurred approximately every 10 to 12-weeks, such that The Council curricula (treatment condition or experimental group) could be completed during the 10 -weeks in between measurements. The administration of the survey occurred during a week in which the program was not implemented. The participants who did not receive The Council curricula were considered the control group or the comparison condition. The study does not utilize a true experimental design, because the youth were not randomly assigned to treatment or control. However, a quasi-experimental design is still present because four pre-existing groups (ODYS facilities) were assigned to the two different conditions. Because random assignment to treatment and control conditions did not take place, analyses were conducted to assess the equivalency of the four sites and across the two conditions.

\section{Measures}

The current study utilized a number of measures from the larger study. The measures for the original study (see Appendix C) included those that assessed 
demographic information, school engagement, gang involvement, positive selfimage and social engagement, masculine identity, caring and cooperative behaviors, ethnic identity, self-efficacy, decision-making regarding criminal behavior, and satisfaction with The Council. Furthermore, the ODYS provided records of the youth including their felony, reading and math skills level, and other risk assessment. The measures listed in detail below were used in the present study.

Demographic variables. Demographic items included nine questions about various aspects of the youths' lives and identity including the last three digits of their ODYS identification number, their age, birth date, race/ethnicity, language, living situation before ODYS, and whether or not they had previously lived in a group home. Two of the time-invariant predictor variables in the analytic model, age and racial/ethnic identity, were assessed using responses to these questions. Before these variables were included in the analytical model they underwent a process that involved assessment, computation, and imputation. In the two paragraphs to follow, I describe the steps I took to create an aggregated time-invariant variable for each of the demographic variables.

To begin, age was missing at baseline for 607 participants. For those participants, age was calculated using the birth date provided at that occasion of measurement $(n=2)$. For the remaining 605 participants, age was imputed from survey occasions two or three. All participants $(n=1,248)$ had provided some documentation of age at one survey occasion, so ODYS records were not used. 
What I next assessed was the variable of race/ethnic identity. If the participant's self-report of race/ethnic identity was missing at baseline, race/ethnic identity was assessed at the second, followed by third, occasion of measurement where it was not missing. For the majority of participant's $(n=1,188)$, response to the item assessing race/ethnic identity remained identical across each response. For these participants, the invariant response were aggregated into one variable assessing race/ethnicity at one time-point. Of the remaining 60 participants, ten participants identified as "White" only at one survey occasion, but as "White" and "Other" and wrote in "Irish", "German", "Polish", or "Italian" at another survey occasion. These ten participants were coded as "White" for the aggregate time-invariant variable. One participant identified as "Latino" at one survey occasion and "Latino" and "Other" and wrote in "Hispanic" at another survey occasion. This participant was coded as "Latino" for the aggregate time-invariant variable. Eight participants identified as "African American" at one survey occasion, but "African American" and "Other" and wrote in "Black" or "Blackness" at another survey occasion. These eight participants were coded as "African American" for the time-invariant aggregate race/ethnicity variable. The remaining 41 participants had no clear pattern of a single race/ethnic identity, and were thus coded into the "Other" race/ethnic category. Finally, a second step was taken to prepare the race/ethnic identity variable for analysis. Using responses aggregated into the one time-invariant variable, four new time-invariant variables were created for the four dichotomous race/ethnic identity categories assessed in the analytical model. 
ODYS Records. Additional demographic data has been obtained from ODYS institutional records. These records include the number of days the youth has been incarcerated at ODYS, race, the felony they were charged with, their attendance at The Council groups, risk level, level of education, reading and math scores, and whether they have a high school diploma or GED.

As was done with age and race/ethnic identity described above, days in prison underwent a process of assessment and imputation in order to create one aggregated time-invariant variable to be included in the model. Specifically, number of days in prison was calculated by taking the number of days in prison that was provided during a different occasion of measurement in the study. The same reference date was used for all occasions of measurement, thus, the single number provided at any time-point can be utilized for the aggregate variable. Of the 1248 participants in the current study, 876 had at least one record provided by ODYS. The remaining 372 did not have a record for any time-point. For these participants, the number of days they have been in prison is unknown. The analyses using this variable will have a decreased sample size of 876 .

Adolescent Masculine Identity in Relationships Scale. The Adolescent Masculinity Ideology in Relationships Scale (AMIRS; Chu, Porche \& Tolman, 2005) is a 12-item measure assessing emotional stoicism, heterosexual dominance, sexual "drive," physical toughness, competiveness, and ambition in young men. The AMIRS provides a list of belief statements and respondents are asked to indicate their agreement using a four-point Likert scale, including response options: (1) 
disagree a lot; (2) disagree; (3) agree; and (4) agree a lot. Items that are negatively worded are to be reverse-scored before composite scores are created and before data analysis takes place. The authors propose for composite scores to be calculated by taking the average of the responses to the 12-items. Higher scores represent adherence to more traditional hegemonic masculine ideals.

Internal consistency reliability has been established in studies with middleschool and high-school aged young men (seventh grade: $\alpha=.71$; eighth grade: $\alpha=$ .67 ; high school: $\alpha=.70$ ). Further, the reliability for the combined sample of seventh grade, eighth grade, and high school boys was moderate $(\alpha=.70)$. Concurrent construct validity was determined by the moderate correlation scores on the AMIRS with two other established measures that represent traditional views of masculinity (Chu et al., 2005).

The outcome scale to assess masculinity ideology (AMIRS; Chu et al., 2005) has undergone an assessment of its psychometric properties for the current sample. To begin, five of the 12-items were reverse-coded such that high scores represent greater adherence to traditional masculine ideals on all items. Second, the internal consistency reliability using Cronbach's alpha was assessed at each time point (Table 7b). For each survey occasion, internal consistency reliability $(\alpha=.744 ; .723 ; .727)$ was above that reported by Chu and colleagues (2005) $(\alpha=.67)$ and fell in range of levels that have been deemed acceptable (George \& Mallery, 2003). Moreover, examination of "Chronbach's alpha if item deleted" did not suggest a consistent method of improving the survey's internal consistency. Specifically, deleting an item 
from the first survey occasion would not have improved the level of Cronbach's alpha. However, for the second and third survey measurement occasions, Cronbach's alpha would have been improved slightly if item D11, "It's embarrassing for a guy when he needs to ask for help," (see Appendix C) was deleted. Finally, examination of the corrected-item total correlation statistics revealed a range of correlation coefficients ( $r=.161$ to .507 ) with the majority greater than $r=.3$. Taken together, this information suggests that although there are lower than desired correlation coefficients between some items (inter-item correlation matrix) and among some items in relation to the rest of the scale, no item was problematic enough in a consistent way that would improve the scale if it were deleted. For these reasons, the 12-item scale remained fully intact as suggested by the authors (Chu et al., 2005).

An additional examination of the outcome measure was made to assess time of measurement non-response and the scale non-response. Time of measurement non-response $(n=602 ; 578 ; 617)$ remained relatively consistent over the three survey measurement occasions. Additionally, scale item non-response was minimal with only two participants completing less than $70 \%$ of the items at the initial and final survey measurement and only one participant at the second survey measurement occasion. For these participants, a composite scale score was not created. For the remaining participants, an average composite score was computed across the 12-items. The means and standard deviations for the scale are provided in Tables 7a-b. 
Ethnic Pride. Ethnic pride was assessed as a proxy for ethnic belonging using one item from the Ethnic Identity - Teen Conflict Survey (EI-TCS; Bosworth \& Espelage, 1995). The EI-TC consists of 4 items measuring ethnic pride and respect for ethnic differences. The EI-TC asks respondents to indicate how often they would make a statement on a 5-point Likert scale: (1) never; (2) seldom; (3) sometimes; (4) often; (5) always. At face value, only one item of the scale measures ethnic pride, whereas the others assess respect for ethnic differences. The one item states, "I am proud to be a member of my racial/cultural group." Thus, ethnic pride was assessed using this item only.

The majority of the participants $(n=717)$ in the current study responded to the item regarding ethnic pride during at least one survey occasion. Several participants $(n=328)$ responded to this item at two of the three survey occasions and fewer $(n=184)$ at all survey occasions. Finally, a small number $(n=19)$ of the sample did not respond to this item at any survey occasion. Thus, these participants will be excluded from the analytical model assessment for this component and for additional model building analyses if this is a significant contributor to the model.

After assessing scale/time of measurement non-response, a one-way repeated measure Analysis of Variance (ANOVA) was conducted to determine whether youths' level of pride changed across the three survey occasions. Descriptively, ethnic pride was strongest at initial survey measurements $(M=3.11, S D=1.357)$, followed by the second survey measurement $(M=3.06, S D=1.344)$, and the last survey measurement $(M=2.9, S D=1.423)$ (see Tables 7a-b). However, results of 
the ANOVA indicated that change across survey measurement occasion was nonsignificant; Wilk's $\Lambda=.999, F(2,181)=.062, p=.939$, multivariate $\eta^{2}=.001$. These results suggest that youths' level of ethnic pride remains stable over the course of 24 weeks. Given the stability in responses to this item, an aggregate time-invariant variable was computed by taking the average response across the three survey measurement occasions.

Satisfaction with The Council. Four open-ended items are included at the end of the post- and follow-up survey instruments for youth participating in The Council at the experimental locations. These four items were created by Beth Hossfeld, The Council director, with the aim of assessing program effectiveness. The four items assessed youths' satisfaction and participation with The Council. The four-items are: S8, “What have you learned in Boys \& Young Men's Council?”; S9, What have you learned about being male?"; S10, “What have you liked and/or disliked about Boys \& Young Men's Council?”, and S11, "Have you changed in any way after being a part of Boys \& Young Men's Council?’. The open-ended qualitative responses to these items were used to better assess how The Council affects youths' experience of gender, that of being male.

\section{Data Analysis}

In the sections to follow, I discuss the steps I took to prepare and analyze the data for evaluation of the research questions and hypothesis tests. To begin, I describe the process I used to analyze and prepare the data for examining Research Question 1. In this section, I describe the following: (1) data screening and data 
structure; (2) results of an a priori statistical power analysis; (3) the steps I took to descriptively assess and report the patterning of change over time; (4) assessment of missing data and the implications to this study; (5) the steps I took to assess the assumptions of Multilevel Modeling; and finally, (6) the procedure and justification of the iterative process of Multilevel Modeling that I took to determine a final model. Second, I describe the process I used to prepare the qualitative data for evaluation of Research Question 2 before describing the results of the hypothesis testing.

\section{Research Question 1.}

Data Screening and Data Structure. Before analyzing the statistical model of the dissertation, the original data file was screened for data entry errors, outliers, and collinearity. First, I examined the frequency distributions of the variables included in the model to assess for data entry errors. Two errors were detected as being out of range and were verified against the participant's completed hard survey and reentered correctly into the database. Additionally, outliers were assessed univariately by inspecting frequency distributions of scores greater than three standard deviations beyond the mean. Ten cases were detected in the variable assessing days in prison that were three or more standard deviations from the mean (> 1,520 days). These ten cases were examined and determined to be reliable given the age of the youth ( $\geq 17$ years) and the possibility of being in the system since they were 12 years old. For this reason, these data were not excluded from analyses. Collinearity was assessed by calculating the squared multiple correlation between each different predictor variable in the model as the criterion and the remaining 
variables in the model as predictors. To begin, age was set as the criterion variable, with days in prison, ethnic pride, and three of the four race/ethnicity variables (African American, Latino, and White) as predictors. The model predicted $12 \%\left(\mathrm{R}^{2}\right.$ $=.122$ ) of the variance in age. This same assessment was conducted for the other two continuous variables with the following results: Ethnic pride: $\mathrm{R}^{2}=.023$; Days in Prison: $\mathrm{R}^{2}=.166$. Additionally, Tolerance, $1-\mathrm{R}^{2}$, and Variation Inflaction Factor (VIF), $1 /\left(1-\mathrm{R}^{2}\right)$, was assessed. Results, age $($ Tolerance $=.878, \mathrm{VIF}=1.14)$, ethnic pride $($ Tolerance $=.977, \mathrm{VIF}=1.02)$, and days in prison $($ Tolerance $=.834, \mathrm{VIF}=$ 1.20), reveal Tolerance values greater than .10 and VIF less than 10, indicating no problems of collinearity.

Given the overlap with The Council indicator variable, variables indicating the prison facility in which the youth resides were not included in the model. Specifically, all youth at Circleville and Ohio River Valley were in The Council and all youth at Cuyahoga Hills and Indian River were not in The Council. Thus, including both location variables and The Council variable would be redundant. Multilevel Modeling

Multilevel Data Structure. In order to address the longitudinal structure of the data, I assessed the hypotheses of the first research question using multilevel modeling. Multilevel modeling allows for comparisons to be made between persons (Level-2) and within persons (Level-1). For the current study, the level-1 variables represent each individual's three waves of data spaced approximately 10 to 12 -weeks 
apart. The level-2 variables include individual characteristics (e.g., age, race/ethnic identity, ethnic pride, days in prison, and participation in The Council).

General Data Structure. In order to maximize the longitudinal nonrandomized experimental design of the study, only some occasions of data were utilized. For the control site data from Indian River and Cuyahoga Hills, all three measurements were used as baseline control measures. For Ohio River Valley, only the first three measurements were used. The first represents a baseline measure before the introduction of The Council. The second two measures represents followup measures, the first approximately 10-weeks from baseline, and the second approximately 20 -weeks from baseline. For Circleville, the last three waves of measurements were utilized as the first three represented baseline data. Thus, Circleville mirrors Ohio River Valley in the experimental condition. Finally, the physical data structure was constructed in PASW statistics version 18.0 (2009) as a person-period data set (Singer \& Willett, 2003) such that each participant had a unique row of data for each data collection period. In other words, each participant had three rows of data that arrange their empirical growth record vertically.

Additionally, because race/ethnic identity is a categorical variable and cannot be directly entered into a regression model and meaningfully interpreted, this variable was converted into four dichotomous variables using dummy coding. As was illustrated in the participant section, the majority of the sample identifies as African American, followed by White, Latino, and some combination of identities or other. Thus, ethnicity is represented with four variables on behalf of the three distinct 
ethnicities included in the sample and one category for those who identify with multiple categories or other, where a score of " 1 " indicates the participant identifies with that ethnicity, and " 0 " indicates the participant does not identify with that ethnicity. African American served as the base category for multilevel analyses because this category has the largest number of participants in the current sample. Each participant has a code of " 1 " in one of the four variables and " 0 " in all others because all those who circled more than one ethnicity were grouped into "Other." For purposes of analyses, all categories of race/ethnicity were incorporated into the statistical model. However, the category of "Other" is not assessed and interpreted with the other categories, as there is no meaningful interpretation of this group together. Alternatively, a description of the group heterogeneity with regards to race/ethnic identity responses is provided to inform future research endeavors.

As a function of the longitudinal design and the nature of the Ohio Department of Youth Services system, some participants moved from one location to another during the study. These participants were removed from the study as they may contaminate the experimental design and the potential location effect. The number of cases removed for this reason was reported in the Methods section.

Statistical Power. A power analysis was conducted using Optimal Design Software (Liu, Spybrook, Congdon, Martinez, \& Raudenbush, 2009). The analysis assumed random assignment, “orthogonal designs, continuous outcomes, a linear link function, random effects covariance structure, homogenous covariance structure within each treatment, and complete data." (Spybrook, Raudenbush, Congdon, \& 
Martinez, 2009, p. 44). Because the study does not meet all of these assumptions (e.g., random assignment, complete data) the analysis resulted in a biased and overly optimistic estimate.

Using Optimal Design for repeated measures trials, a power analysis for treatment on linear change was conducted. Specifically, given the sample size of the current study is fixed and known, an analysis of the power vs. effect size was examined. The individual-level sample size $(\mathrm{J})$ was set to 1,248 and the repeated measures sample size (n) was set to 3 , though it should be noted that most $(85 \%)$ individuals in the sample do not complete all three measurement occasions. Alpha was set to .05 , such that the probability of making a type I error (rejecting the null hypothesis when it is in fact true), was minimal. This same critical value was utilized in the analyses of the study hypotheses.

Given the sample size, alpha, an Intraclass Correlation Coefficient estimate of $\rho=.1$ and $\rho=.5$, and power of .8 , it was estimated that the current study will be able to detect an effect size between $d=.1$ and $d=.15$. According to Cohen (1988), the size of effect can be categorized as small. The level of power was selected to limit the probability of making a type II error (failing to reject the null hypothesis when it is in fact false). Power $=.8$, results in a probability of making a type II error of $\beta=.2$.

Exploring Empirical Growth. Before analyzing the statistical model, I visually assessed individual change over time by examining empirical growth plots (Singer \& Willett, 2003). I selected approximately 40 individuals from the 
experimental group (20 from each location) and 40 from the control group (20 from each location) and plotted occasion of measurement by the outcome measure, adherence to traditional masculinity ideology (see Graphs 1-4). I examined the nonparametric growth trajectories individually for each location and in comparison to the others. Overall change over time appears to be small and varied. Whereas some individuals show an increased adherence to traditional masculinity ideology over time, others show a decrease.

Centering. Centering variables eases the interpretation of findings such that the zero-point becomes the average of that variable (Enders \& Tofighi, 2007). For the continuous variables, age and days in prison, zero is less meaningful than the average. Thus, these level-2 variables were grand mean centered. The grand mean age of the sample is 16.83 years and the grand mean number of days in the Ohio prison system is 349.14 . Thus, these two mean points were set equal to zero and deviations greater than the mean were positive and those less than the mean were negative. Additionally, ethnic pride was assessed using one item on a 5-point Likert scale. Thus, ethnic pride was also grand mean centered such that zero becomes a meaningful point of interpretation. The grand mean level of ethnic pride is 3.00, which was set to zero with deviations above the mean represented with positive numbers and below the mean with negative numbers. For all analyses reported in the Results Chapter of this dissertation, age, days in prison, and ethnic pride were grand mean centered. 
Missing Data. Due to contextual factors within the ODYS facilities and the staggered research design, several participants in the current study are missing responses from one or more surveys. It should be noted, even in less complex designs missing data is a common problem in psychological research. With that said, longitudinal studies are especially susceptible to missing data. Researchers have examined several problems associated with missing or incomplete data. Most importantly, studies with incomplete data suffer from a loss of statistical power and may be biased because of potential differences between observed and missing values (Cole, 2008).

In order to appropriately deal with missing data, a few assumptions must be considered. In 1976, Rubin created a taxonomy of missing data mechanisms: Missing Completely at Random (MCAR); Missing at Random (MAR); and Missing Not at Random (MNAR). To begin, MCAR, refers to the situations in which missingness on a variable is unrelated to the values of other variables including the variable that is missing. Second, MAR, which is less restrictive than MCAR, refers to situations in which missingness is related to one or more other observed variables in the model, but unrelated to the values of the variable that is missing. Lastly, MNAR refers to the situation in which missingness on a variable is dependent on the values of that variable. In longitudinal designs, MCAR refers to situations in which the probability of dropout (attrition) is unrelated to any characteristics of the participant, MAR refers to situations in which the probability of dropout may be related to pre-dropout responses, and MNAR is when the probability of dropout is related to responses at 
the time of dropout (Schafer, 2005). In longitudinal multilevel modeling, data are most often MAR (Hox, 2010), and in the study MAR is assumed.

In order to determine the extent of missingness in the current study, I first counted the number of surveys that could have been completed at any given location or wave of the study. Next, I counted the total number of surveys that were actually completed. I then divided the total number of completed surveys by the total number of possible surveys to obtain a percentage of completion. This resulting number represents the percentage of overall compliance in the current study (48.73\%). Further, in order to determine the percentage of compliance at each wave of the study, I divided the number of completed surveys by the number of possible surveys for each wave $(20.89 \%-67.50 \%)$ (see Table 8$)$.

Next, I examined the extent of missing data within each predictor variable (see Tables 3 and 7b). For some of the variables, for example, age, multiple sources of data were used to determine the participant's data estimate (e.g., age at baseline), and therefore missing data is minimal for these variables. For others, however, only one data source could be used and thus, missing data is more problematic. Of the 1,248 participants in the study, age at baseline is known for 1,243 (99.6\%), the number of days in prison for 876 (70.2\%), race/ethnic identity for 1,245 (99.8\%), ethnic pride for $1,229(98.5 \%)$, attendance for the first 10 -weeks $(n=218)$ and the second 10-weeks $(n=169)$. The outcome, repeated measure of masculine ideology, also suffers from missing data, where data estimates exist for $644(51.6 \%)$ at baseline, $669(53.6 \%)$ at time 2, and $629(50.4 \%)$ at time three. To be clear, a data 
estimate for the outcome measure is known for each of the 1,248 participants during at least one wave of the study. Given the different amounts of missingness in the study variables, I carefully assessed the model with and without the variables most affected by missing data, which I thoroughly described in the results section to follow.

Next, I assessed whether each predictor variable in the model at a given survey occasion predicted missing data on that variable for the other survey occasions. To begin, I created a missingness variable using dummy coding, to code for missing (0) and non-missing (1) for each variable at each wave. Then, each missingness variable was regressed on its actual variable at a different survey occasion to determine whether the probability of missingness on the variable is not related to the participant's score on that variable (see Tables 9a-d). The impact of these findings are described in the limitations section of the Discussion Chapter.

Finally, missing data of the predictor variable, days in prison, cannot utilize the procedure of "imputation from another variable" and thus, was dealt with in the following way. First, I contacted staff members at ODYS and requested data for the variable "days in prison" for the cases in which it was missing. Because I was unsuccessful in attaining these additional records, the participants without data on this variable were excluded from analyses using this variable.

Assumptions. (1) Assumption of Linearity. A visual inspection of the bivariate scatterplots of the variable combinations of interest in the model was examined in order to assess if a linear relationship is present. Specifically, bivariate scatter plots 
of each continuous predictor variable against the outcome variable, masculinity ideology, were assessed. (2) Assumption of Normality. The assumption that the level1 residuals and level-2 random effects in the model are distributed normally was assessed through a visual inspection of histograms of the residuals and the normal probability plots. Level-1 residuals were created from the complete model (Model 1) to be described in detail in the Results chapter. Inspection of the level-1 residual histogram revealed a normal distribution around 0.0 residual. Inspection of the normal probability plot of the level-1 residuals also indicated normal distribution with points falling closely to the line. (3) Assumption of Homoscedasticity. The assumption that the level-1 residual variance is constant was assessed in the visual inspection of the histogram of the residuals and the scatterplot of level-1 residuals and predicted values. Inspection of the scatterplot indicates a slight positive and linear relationship between the residuals and predicted values, though the general trend of the data appears to be a "blob" and thus, is indicative of a homogenous distribution of errors across all values of the predictor variables. (4) Assumption of Independence: Collinearity of level-2 predictors was assessed as described earlier in order to assume independence of observations at level-2. Additionally, bivariate correlations were assessed among all predictor variables. Specifically, Pearson Correlation coefficients were examined for relationships among two continuous variables, Point Biserial Correlation coefficients were examined for relationships among a continuous and a dichotomous variable, and Phi Correlation coefficient was examined for relationships among two dichotomous variables. Age is significantly 
related to days in prison, ethnic pride, and White race/ethnic identity. Specifically, older youth tended to be in ODYS longer, have higher levels of ethnic pride, and tended to be White. Additionally, days in prison is related to White race/ethnic identity, and The Council, such that White youth tended to be in the system for less days and in the experimental locations (Ohio River Valley and Circleville) participating in The Council. Latino youth report higher levels of ethnic identity compared to non-Latino youth. Finally, non-White youth tended to be in the control locations (Cuyahoga Hills and Indian River), not participating in The Council. For a full correlation matrix of level-2 predictor variables see table 10. The assumption of independence may be in question given the correlations among some of the level-2 predictor variables. (5) Reliability of Predictor Variables. Multilevel analysis assumes that the predictor variables are measured perfectly reliably. Unfortunately, not all predictor variables can be assessed for reliability. For example, days in prison is measured once by the ODYS facility staff. It is indicated by a single item and as such cannot be determined to be perfectly reliable. However, both age and ethnic status, two other important predictors in the statistical model, were assessed via responses from the youth participants in addition to the facility's records. In comparing the two sources of data, I assessed measurement error for these two predictor variables. (6) Omitted Predictors are Uncorrelated with Variables in the statistical Model. The variables selected for this model were determined based on an extensive review of the literature. As is noted in the limitation section of this 
dissertation, other potentially important predictor variables have not been assessed and should be considered in future research.

Multilevel Modeling. I hypothesized a complex multilevel model, made up of several different theoretically important parameters, interaction effects, cross-level interactions, and multiple random effects. Unfortunately, the complexity of this model may made reaching convergence difficult. If convergence were reached with the full model, hypotheses would have been evaluated against the results of this model. However, the estimation procedure failed to converge on the full model specified, including all hypothesized parameters, so I engaged in a process of model trimming. Given the theoretical importance of all hypothesized model parameters, I used the following guiding principles to reach a final, parsimonious model that was able to converge. First, when the model failed to converge, I replaced the random slope effects with fixed effects, setting the variance of the random effects equal to zero. Next, I will considered the effect of missing data on some of the predictor variables. As was described above, some predictor variables have more missing data than others. Because the analytic procedure employs listwise deletion, when these parameters were included in the model, the statistical power was reduced. To be clear, when all model variables were included in the full model the sample size is reduced to 876 . This reduction in statistical power influenced whether a parameter statistically contributed to the model. Therefore, parameters affected by the reduced sample size (i.e., all parameters that include the indicator of number of days in prison) and not statistically contributing to the model were removed and I compared 
the overall fit of the reduced model to the previous, full model. In particular, I removed each model parameter that includes the variable number days in prison, one at a time, which eventually increased the sample size to 1,229.

Research Question 2: What do youth who participate in The Council learn about being male?

Research question 2 was assessed using "thematic analysis" (aka "content analysis"), a qualitative method for identifying, analyzing, describing, and reporting patterns within data (Braun \& Clarke, 2006; Spencer, Ritchie, O’Connor, 2003). I followed Braun and Clarke's definitions of terms to describe the data to be analyzed using this methodology. The data corpus, all the data collected for this research project, includes the participant paper-and-pencil responses and ODYS records data. The data set includes all of the open-ended qualitative paper-and-pencil responses that may be analyzed in addressing this particular research question. The data item refers to each response to a qualitative open-ended question. To be clear, only participant's at Ohio River Valley and Circleville, the experimental locations, at post- and follow-up survey occasions were given the opportunity to respond to these open-ended questions. Additionally, there were four open-ended survey items. However, only one of the items directly assesses the construct of masculinity. Thus, all responses to the item S9, What have you learned about being male?", were included in the data items. In addition, all responses to the other three items, S8, “What have you learned in Boys \& Young Men's Council?"; S10, “What have you liked and/or disliked about Boys \& Young Men's Council?”; and S11, “Have you 
changed in any way after being a part of Boys \& Young Men's Council? " that describe something related to masculinity ideology were included. Therefore, the total possible number of data items will be the product of the number of participants from Ohio River Valley and Circleville at post- and follow-up times the four possible open-ended survey responses. Finally, the data extract will include all coded data that will be extracted from a data item for the purposes of answering the research question (RQ2) described above.

The purpose of this thematic analysis of the qualitative, open-ended survey responses was to inform the predominantly quantitative investigation into masculinity ideology among adolescent inmates over time. I followed Braun and Clarke's 6-phases of thematic analysis: (1) Gaining familiarity with the data; (2) generating initial codes; (3) searching for themes; (4) reviewing themes; (5) defining and naming themes; and (6) producing the report. In the paragraphs to follow, I describe the steps I took to fulfill each of these phases of inquiry and analysis.

Phase 1: Gaining familiarity with the data: In the first phase, I managed the data by first identifying the data set and data items. Given the goal of this particular analysis stated above, I first identifed and carefully select the data items based on the following criteria described above. After all data items were selected, I read, and reread the selected data to familiarize myself with the content. During this time, I took notes on my initial thoughts and responses to the data.

Phase 2: Generating initial codes: In the second phase of analysis, I coded for features of the data that inform the research question posed. To be clear, during 
this phase I made notes of codes next to the data extracted. These initial codes were then constructed into a manageable list (e.g., Conceptual Framework or Index;

Ritchie, Spencer, \& O’Connor, 2003), so that the initial codes were prepared to be organized and grouped in the third phase.

Phase 3: Searching for themes: Once all of the data has been initially coded and constructed into a conceptual framework list, I began the phase of code analysis where the broader level themes were identified. I utilized tables and "mind-maps" to visually represent the different initial codes into themes. At the end of this phase, I was left with a collection of all possible themes and sub-themes.

Phase 4: Reviewing themes: During phase 4, I made organizational decisions about the themes. Some themes were subsumed by others, some were collapsed into one, some will be broken down into separate themes, and still others were deleted. These decisions were made based on the nature of the data within the themes. For example, if the data within a theme were similar and are clearly distinct from data under other themes, the theme was kept. If, on the other hand, the theme does not appear to have a coherent pattern of data, I considered both the theme and the data extracted within the theme and spend time reorganizing the data/theme or creating a new theme. At the end of this phase, I was left with a "thematic map" that captured the patterns of the coded data.

Phase 5: Defining and naming themes: During the fifth phase of analysis, I defined the themes by identifying and naming the theme such that it captured the nature of the data within the theme. For each theme, I described the story the theme 
tells and how it helps to answer the guiding research question. During this phase, I also considered the structure of the themes and redefined themes into subthemes of others, if there is too much overlap. At the end of this phase I had a clear set of themes and subthemes and a definition of what each theme is and what it is not.

Phase 6: Producing the report: Finally, I reported the findings of the thematic analysis with detail and example quotes, such that the process of analysis was transparent, coherent, and logical. In this phase, I link the themes of the qualitative follow-up sequence (e.g., Morgan) to the quantitative findings. Here I used the results of the thematic analysis to inform the results from the inquiry of research question 1 . The goal of this phase was to bring the qualitative findings into conversation with the quantitative findings. This is an extremely important process as the different method (qualitative) of inquiry at follow-up did not fully replicate the quantitative findings (e.g., Ritchie, 2003). Thus, both the overlap and the differences were explored, presented, and discussed. 


\section{CHAPTER VII}

\section{Results}

\section{Research Question One}

Descriptive Information. Averaging across participants and over time, participants tended to "disagree" with statements regarding adherence to traditional masculine ideals $(M=2.21, S D=.428)$. Participants' level of adherence to traditional masculine ideals was assessed using the Adolescent Masculine Ideology and Relationship Scale (Chu et al., 2005), a 4-point Likert scale where low scores (e.g., mean $=1$ ) represent strong disagreement and high scores (e.g., mean $=4$ ) represent strong agreement to traditional masculine norms. Descriptively, participants' level of adherence to traditional masculine ideals increased slightly over time. The lowest average agreement reported at the initial assessment $(n=644 ; M=$ $2.19, S D=.438)$, followed by post-survey $(n=669 ; M=2.22, S D=.419)$, and with highest average level of agreement reported at follow-up $(n=629 ; M=2.23, S D=$ $.427)$.

On average, at baseline participants were 17 years old $(n=1,243 ; M=16.83$, $S D=1.40)$, had been in prison for 473 days $(n=876 ; M=472.87, S D=349.27)$, and were "sometimes" proud to be a member of their racial/cultural group $(n=1,229 ; M$ $=3.0, S D=1.27$ ). As previously described, the measure of ethnic pride (Bosworth $\&$ Espelage, 1995) is assessed on a 5-point Likert scale $(1=$ never; $2=$ seldom; $3=$ sometimes; 4 = often; 5 = always) of how often they would make the statement described above. 
In the experimental locations, participants attended The Council for an average of 14 hours during the first 10 weeks $(n=218 ; M=14.24, S D=6.10)$ and 13 hours during the second 10 weeks $(n=169 ; M=13.07, S D=5.53)$. That is, youth on average participated in $70 \%$ of The Council groups (14 of the possible 20 hours) during the first 10 weeks and $65 \%$ (13 of the possible 20 hours) during the second 10 weeks. As noted in the Methods Chapter, some youth had records with zero hours of attendance recorded. Though these records were included in the assessment of the distribution of this variable for descriptive purposes, they were excluded from the analysis of Hypothesis $1 b$.

As a precursor to hypothesis testing using multilevel modeling, I assessed the intraclass correlation (ICC) by conducting an intercepts-only model $\left(Y_{i j}=\gamma_{0 i}+u_{0 j}+\right.$ $e_{i j}$ ) and calculating ICC with the following formula:

$$
\begin{aligned}
& \text { ICC }=\sigma_{u 0}^{2} /\left(\sigma_{u 0}^{2}+\sigma_{e}^{2}\right) \\
& \text { ICC }=\text { Variance in Intercept } /(\text { Residual Variance }+ \text { Variance in Intercept }) \\
& \text { ICC }=.116501 /(.062380+.116501)=.651
\end{aligned}
$$

The ICC represents the percentage of total variation in traditional masculine ideology scores that is attributed to group membership or, in this case, the variation that is attributed to the individual in which the measurements over time are nested. Thus, $65 \%$ of the variance in level of adherence to traditional masculinity ideology at baseline is due to individual (group) differences. In other words, there is substantial dependency in level of adherence to traditional masculinity ideology as a function of the individual. 
Next, an unconditional growth curve multilevel model, $\left(Y_{i j}=\gamma_{01}+\right.$ $\gamma_{10} \mathrm{OCCASION}_{\mathrm{ti}}+\gamma_{20} \mathrm{OCCASION}_{\mathrm{ti}}+\mathrm{u}_{0 \mathrm{j}}+\mathrm{u}_{1 \mathrm{j}}+\mathrm{u}_{2 j}+e_{i j}$, was computed to examine the growth trajectory of level of adherence to traditional masculinity ideology longitudinally across three survey measurements. The unconditional model represents the level-1 components of the multilevel model, which is also referred to as the individual growth model. There are two parts to the unconditional model, the structural part, which represents the hypotheses regarding each participant's trajectory of change over time, and the stochastic part, which represents the random error in association with measurement error (Singer \& Willett, 2003). The purpose behind estimating the unconditional growth model was to understand the patterning of change in masculine ideology that one would expect to see in the population of adolescent inmates over the course of this study without the addition of predictor variables. This is determined by the structural part of the model. This structural part provides a baseline estimate of initial levels of adherence to traditional masculinity and change over time, which served as a reference point for subsequent models. The stochastic part of the model provides an estimate of how much variation occurs between individuals in their initial levels of adherence and change over time (slopes). Additionally, the stochastic part of the model provides an estimate of how much variation is left unexplained by the model. As predictor variables are included in the model, these stochastic elements change and can be compared to the estimates of the unconditional growth model to determine the effect of the addition of predictor variables on initial levels of adherence to traditional masculine ideals, change over 
time, and whether the addition of the predictor variables helps explain more variation in the data. In other words, the unconditional growth model provides a starting point in which all subsequent models were compared.

However, convergence was not achieved for this unconditional growth model, and model estimates were stopped at the $37^{\text {th }}$ iteration. Model convergence and the number of iterations needed to reach convergence are both diagnostics of model to data fit (Raudenbush \& Bryk, 2002). Because convergence was not reached, a second unconditional growth model was assessed. In the second model, the stochastic elements of the slopes were constrained to zero to simplify the model. This second unconditional-only model converged after four iterations. The results indicated that on average level of adherence to traditional masculinity at baseline was $2.20(p<.001)$. Additionally, level of adherence to traditional masculinity did not increase at a level significantly different from zero, with an average of .012 ( $p=$ $.445)$ units for the first 10-weeks and $.024(p=.174)$ units over the course of the study, approximately 20 weeks. The random variance of level of adherence to traditional masculinity was significant at the intercept $\tau_{00}=.116, p<.001$, such that youth had varying initial levels of adherence to traditional masculinity ideology. Finally, random variability .063 $(p<.001)$ was left unexplained by this model, a small fraction of which is explained by the additional variables included in the models presented below.

Given the linear description of change that was determined both visually and through inspection of the means, an additional unconditional growth curve multilevel 
model was computed to examine the linear growth trajectory of level of adherence to traditional masculinity ideology, $\left(Y_{i j}=\gamma_{01}+\gamma_{10}\right.$ OCCASION $\left._{\mathrm{ti}}+\mathrm{u}_{0 \mathrm{j}}+\mathrm{u}_{1 \mathrm{j}}+e_{i j}\right)$. The results of the second unconditional-only model indicated that on average level of adherence to traditional masculinity ideology at baseline was $2.19(p<.001)$ and that level of adherence descriptively increased, though nonsignificantly, linearly by an average of .012 ( $p=.163)$ units every 10-weeks. The intercept variance of level of adherence was significantly different from zero, $\tau_{00}=.14, p<.001$, indicating that baseline adherence varied significantly across participants. However, change in adherence did not vary significantly across individuals, $\left(\tau_{10}=.004, p=.221\right)$.

Finally, the intercept and slopes were negatively related at a non-significant level $\left(\tau_{11}\right.$ $=-.011, p=.204)$, such that participants with high levels of initial adherence tended to increase less than those with initially low levels of adherence.

\section{Multilevel Modeling}

In order to assess each hypothesis under the first research question, I engaged in an iterative process of model trimming so that a final analytical model could be selected. The goal behind this process of model trimming, as described earlier, was to end with a final model that achieves convergence and can best describe the effects of each parameter. To be clear, a full model was and would be retained as the final model if the model achieved convergence and if missing data were not a problem. However, as described in the previous chapter, the model is complex and some parameters (e.g., days in prison) are more affected by missing data than others. Thus, when the full model failed to converge, I fixed the two random slope effects equal to 
zero. Next, in order to explore the effect of missing data, I fixed parameters highly affected by missing data equal to zero. For example, all parameters that include the variable, number of days in prison, are highly affected by missing data. Therefore, the removal of these parameters would increase the statistical power and the ability to detect an affect of one of the other model parameters.

In the following paragraphs, I explore several models to determine which model best fits and explains the data. First, the full model, including all predictor and control variables included in the study hypotheses, is presented. In the subsequent steps, I describe how a trimmed model was selected and assessed and compared to the previous one. In all, seven models were assessed, each nested within the complete model described in detail below. After describing the results of the complete model and the comparison of each nested model, I conclude with a detailed description of the results of the final model. After the results of this model are fully described, I review each hypothesis and describe how the model results inform these hypotheses.

Complete model:

$Y_{i j}=\gamma_{0 i}+\gamma_{10} \mathrm{OCCASION}_{\mathrm{ti}}+\gamma_{20} \mathrm{OCCASION}_{\mathrm{ti}}+\gamma_{01} \mathrm{COUNCIL}_{i}+\gamma_{02} \mathrm{AGE}_{i}+$ $\gamma_{03} \mathrm{DAYS}_{i}+\gamma_{04} \mathrm{WHITE}_{i}+\gamma_{05} \mathrm{LATINO}_{i}+\gamma_{06} \mathrm{OTHER}_{i}+\gamma_{07} \mathrm{PRIDE}_{\mathrm{ti}}+$ $\gamma_{11} \mathrm{OCCASION}_{\mathrm{ti}} * \mathrm{COUNCIL}_{i}+\gamma_{21} \mathrm{OCCASION}_{\mathrm{ti}} * \mathrm{COUNCIL}_{i}+$ $\gamma_{31} \mathrm{OCCASION}_{\mathrm{ti}} * \mathrm{AGE}_{i}+\gamma_{41} \mathrm{OCCASION}_{\mathrm{ti}} * \mathrm{AGE}_{i}+\gamma_{51} \mathrm{OCCASION}_{\mathrm{ti}} * \mathrm{DAYS}_{i}+$ $\gamma_{61} \mathrm{OCCASION}_{\mathrm{ti}} * \mathrm{DAYS}_{i}+\gamma_{71} \mathrm{OCCASION} 1_{\mathrm{ti}} * \mathrm{WHITE}_{i}+$

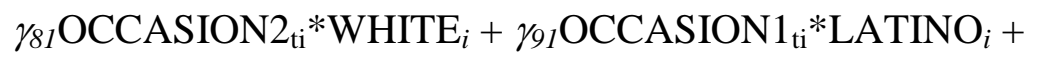


$\gamma_{10.1} \mathrm{OCCASION}_{\mathrm{ti}} * \mathrm{LATINO}_{i}+\gamma_{11.1} \mathrm{PRIDE}_{t i} * \mathrm{WHITE}_{i}+\gamma_{12.1} \mathrm{PRIDE}_{t i} * \mathrm{LATINO}_{i}+$ $\gamma_{13.1} \mathrm{PRIDE}_{t i} * \mathrm{OCCASION}_{\mathrm{ti}}+\gamma_{14.1} \mathrm{PRIDE}_{t i} * \mathrm{OCCASION}_{\mathrm{ti}}+\gamma_{15.1} \mathrm{COUNCIL}_{\mathrm{i}} * \mathrm{AGE}_{i}$ $+\gamma_{16.1} \mathrm{COUNCIL}_{\mathrm{i}} * \mathrm{DAYS}_{i}+\gamma_{17.1} \mathrm{PRIDE}_{t i} * \mathrm{OCCASION}_{\mathrm{ti}} * \mathrm{WHITE}_{i}+$ $\gamma_{18.1}$ PRIDE $_{t i} *$ OCCASION $_{\mathrm{ti}} * \mathrm{WHITE}_{i}+\gamma_{19.1}$ PRIDE $_{t i} * \mathrm{OCCASION}_{\mathrm{ti}_{1}}$ LATINO $_{i}+$ $\gamma_{20.1} \mathrm{PRIDE}_{t i} * \mathrm{OCCASION}_{\mathrm{ti}} * \mathrm{LATINO}_{i}+\gamma_{21.1} \mathrm{OCCASION}_{\mathrm{ti}} * \mathrm{COUNCIL}_{i} * \mathrm{AGE}_{i}+$ $\gamma_{22.1} \mathrm{OCCASION}_{\mathrm{ti}} * \mathrm{COUNCIL}_{i} * \mathrm{AGE}_{i}+\gamma_{23.1} \mathrm{OCCASION}_{\mathrm{ti}} * \mathrm{COUNCIL}_{i} * \mathrm{DAYS}_{i}+$ $\gamma_{24.1} \mathrm{OCCASION}_{\mathrm{ti}} * \mathrm{COUNCIL}_{i} * \mathrm{DAYS}_{i}+u_{0 i}+u_{1 i}+u_{2 i}+e_{t i}$

Convergence of the complete model was not achieved and incomplete results were produced after 100 iterations. To simplify the model in an attempt to reach convergence, the random effects of each occasion of measurement (slope) were set equal to zero (Raudenbush \& Bryk, 2002) as was done with the unconditional growth model, and the model was assessed again. The simplified model $(\mathrm{n}=876)$ converged after four iterations and complete results are provided (Complete Model, Model 1) in table 11a. The models to follow will be presented in Tables 11b-11g, beginning with the full model and ending with the most simple, final model, Model 7. Model 1 (Complete Model) had a -2 Log Likelihood of 1153.98 with 36 parameters in the model. Given the lack of statistical support for several of the model parameters and the problem of missing data in the parameters that include the variable number of days in prison, all model parameters including this variable were removed. The reduced model $(n=1,229)$ was reassessed and converged after four iterations (see Model 7, table 11g). Model 7 had a -2 Log Likelihood of 1529.21 with 30 parameters. The reduced model is nested within the complete model and could be 
compared statistically if the sample sizes were equivalent, which is not the case here. The chi-square test comparison statistic assumes the same sample size for the two models to be compared. Therefore, the model fit statistic produced by Model 7 with the reduced sample $(\mathrm{n}=876)$ (selecting for participants with non-missing data for Days in Prison) was compared to Model 1. The second assessment of Model 7, using the reduced sample, produced a -2 Log Likelihood of 1158.16. The difference between the two models with the same sample size was 4.18 on 6 parameters, which was assessed on a Chi Square distribution with 6 degrees of freedom. The resulting statistic $\left(\chi^{2}(6)=4.18, p=.652\right)$ indicated that the reduced model using the same sample did not fit significantly worse than the complete model. The lack of statistical support for the parameters involving days in prison and the lack of decrease in model fit when these parameters were removed (using the same sample) lends support to retaining the reduced model as the final model. However, in order to fully support the decision of retaining the reduced model (model 7) as the final model, I also conducted a detailed assessment of the five models in between model 1 and model 7 , below. Next, I explored the effect of the decreased sample and power if I were to retain the full model.

Because the reduced model is different from the full model by six parameters (6 df), I assessed the five different possible models in between the full model (Model 1) and the reduced model (Model 7) and compared each nested model to the previous model (see Tables 11a-11g). The sample size for the models that included the number of days in prison (Models 1-6) are all equal $(n=876)$ and therefore, only one 
test was conducted to assess each difference. To describe this process briefly, I began by removing the most complex (e.g., three-way interactions) parameters first, because the complex parameters depend on the single variable, days in prison being included in the model. Therefore, I could not remove the days in prison, single variable before any of the interaction terms. First, I removed one of most complex parameters (three-way interaction) involving the number of days in prison and the second survey wave (Days in prison * The Council * change from 10 to 20 weeks). This reduced model (Model 2) $(-2$ Log Likelihood = 1156.71) did not fit significantly worse than the full model (Model 1) $\left(\chi^{2}(1)=2.74, p=.098\right)$. Next, I removed the second three-way interaction variable, (Days in prison*The Council*change from baseline to 10 weeks) and compared the fit to the previous model. The further reduced model (Model 3) $(-2$ Log Likelihood = 1157.06) also did not fit significantly worse than the previous model $\left(\chi^{2}(1)=0.345, p=.557\right)$. Next, I removed one of the two-way interactions involving change over time (Days in prison*change from 10 to 20 weeks) and compared the fit to the previous model. The further reduced model (Model 4) $(-2$ Log Likelihood $=1157.52)$ did not fit significantly worse than the previous model $\left(\chi^{2}(1)=0.46, p=.498\right)$. Next, I removed the second two-way interaction (Days in prison*change from baseline to 10 weeks). The further reduced model (Model 5) (-2 Log Likelihood = 1157.69) did not fit significantly worse than the previous model $\left(\chi^{2}(1)=0.175, p=.676\right)$. Finally, I removed the third two-way interaction (Days in prison*The Council) involving this variable. The further reduced model, Model 6, $(-2 \mathrm{Log}$ Likelihood $=1158.15)$ did not 
fit significantly worse than the previous model $\left(\chi^{2}(1)=0.459, p=.498\right)$. Lastly, to compare this model to Model $7(-2$ Log Likelihood $=1158.16)$ assessed with the same sample, Model 7 does not fit significantly worse $\left(\chi^{2}(1)=.01, p=.920\right)$ than the nearly identical model with the exception of the predictor variable of the number of days in prison. Given that the fit of the model to the data was not significantly worse in the reduced model, I decided to retain the reduced model, model 7.

In addition to model fit, the explanatory power of the each model iteration was assessed. As seen in Table 11g, some of the model parameters reach statistical significance when the statistical power was improved by removing the parameters that suffer most from missing data. To be clear, the sample included for the first model $(n=876)$ is smaller than that of the final, sixth model $(n=1,229)$. Whereas the first model utilized listwise deletion and therefore used a reduced sample, excluding all participants that were missing data on any variable, the final model was more inclusive, not excluding the participants that were missing data on the days in prison variable because this variable was no longer included in the model. For the reason that this variable suffered the most missing data, the same size increased substantially between the models that included these parameters (models 1-6) and the final model (model 7).

In order to justify the removal of all parameters utilizing the days in prison variable, I further explored the effect of this missing data on the model parameters. First, I assessed the effect of each parameter in the first model compared to the seventh model. Whereas the first model had seven statistically significant fixed 
effects, including the intercept, the seventh and final model had nine. The additional two statistically significant effects have important implications for the theoretical and practical implications of the study's findings. When power was reduced, these effects went undetected. Second, I assessed the difference on all model variables between participants included in the first, full model, to those that were excluded, but included in the seventh model (see Table 12). Excluded participants were statistically significantly older $(t(1241)=6.00, p<.001)$, adhered more strongly to traditional masculine ideals after 10 weeks in the study $(t(667)=3.51, p<.001)$, and were racially/ethnically different $\left(X^{2}(6)=15.38, p=.018\right)$ than those that were included in the first model. For these reasons, the full model is not representative of the entire sample. Finally, I did not engage in further model trimming because no other model variables suffered as greatly from missing data and all specified model parameters are theoretically important to the model. Taken together, the lack of statistical significance for these variables in the model (Model 1), the lack of significant decrease in model to data fit, the effect of the reduction of power on the other model parameters (Model 7), and the group differences in important model variables, I decided to retain the more parsimonious, seventh model as the final model to use to evaluate the study hypotheses. Final model: $Y_{i j}=\gamma_{0 i}+\gamma_{10} \mathrm{OCCASION} 1_{\mathrm{ti}}+$ $\gamma_{20} \mathrm{OCCASION}_{\mathrm{ti}}+\gamma_{01} \mathrm{COUNCIL}_{i}+\gamma_{02} \mathrm{AGE}_{i}+\gamma_{03} \mathrm{WHITE}_{i}+\gamma_{04} \mathrm{LATINO}_{i}+$ $\gamma_{05} \mathrm{OTHER}_{i}+\gamma_{06} \mathrm{PRIDE}_{\mathrm{ti}}+\gamma_{07} \mathrm{OCCASION} 1_{\mathrm{ti}} * \mathrm{COUNCIL}_{i}+$

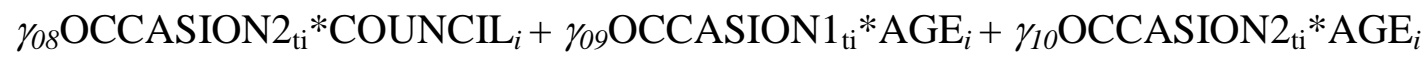
$+\gamma_{11} \mathrm{OCCASION}_{\mathrm{ti}_{\mathrm{i}}} * \mathrm{WHITE}_{i}+\gamma_{21} \mathrm{OCCASION}_{\mathrm{ti}} * \mathrm{WHITE}_{i}+$ 
$\gamma_{31} \mathrm{OCCASION} 1_{\mathrm{ti}} * \mathrm{LATINO}_{i}+\gamma_{41} \mathrm{OCCASION}_{\mathrm{ti}} * \mathrm{LATINO}_{i}+\gamma_{51} \mathrm{PRIDE}_{t i} * \mathrm{WHITE}_{i}+$ $\gamma_{61} \mathrm{PRIDE}_{t i} * \mathrm{LATINO}_{i}+\gamma_{71} \mathrm{PRIDE}_{t i} * \mathrm{OCCASION}_{\mathrm{ti}}+\gamma_{81} \mathrm{PRIDE}_{t i} * \mathrm{OCCASION}_{\mathrm{ti}}+$ $\gamma_{91} \mathrm{COUNCIL}_{\mathrm{i}} * \mathrm{AGE}_{i}+\gamma_{10.1} \mathrm{PRIDE}_{t i} * \mathrm{OCCASION}_{\mathrm{ti}} * \mathrm{WHITE}_{i}+$ $\gamma_{10.2}$ PRIDE $_{t i} *$ OCCASION $_{\mathrm{ti}} * \mathrm{WHITE}_{i}+\gamma_{10.3} \mathrm{PRIDE}_{t i} * \mathrm{OCCASION}_{\mathrm{ti}} * \mathrm{LATINO}_{i}+$ $\gamma_{10.4} \mathrm{PRIDE}_{t i} * \mathrm{OCCASION}_{\mathrm{ti}} * \mathrm{LATINO}_{i}+\gamma_{10.5} \mathrm{OCCASION} 1_{\mathrm{ti}} * \mathrm{COUNCIL}_{i} * \mathrm{AGE}_{i}+$ $\gamma_{10.6} \mathrm{OCCASION}_{\mathrm{ti}} * \mathrm{COUNCIL}_{i} * \mathrm{AGE}_{i}++u_{0 i}+e_{t i}$

\section{Hypothesis Testing}

In the following section, I describe the hypothesis testing of the dissertation study. First, I provide a descriptive summary of each of the hypotheses that fall under the guiding research question described below. Second, I describe the results for each hypothesis. Again, the results can be found in Table 11g, Final Model, Model 7. Hypothesis 1, part b, is assessed with a sub-sample of youth and with a unique model. All other hypotheses were assessed against the results of the final model described above. For this reason, the model and assessment of Hypothesis 1, part $b$ are described at the end of this section. A summary of the results of hypothesis testing can be seen in Table 13.

Guiding Research Question (RQ1): What are the individual and contextual predictors of masculinity ideology development?

Hypothesis 1. Program Effect (see Figure 8a)

Hla: Participation in The Council will predict change in adherence to traditional masculinity ideology. 
Hypothesis 1, part a, posited that participation in The Council will effect the trajectory of change in level of adherence to traditional masculinity ideology. Specifically, it was predicted that change in adherence to traditional masculinity ideology will be negative for youth in the experimental group who have participated in The Council, but not for youth in the control group. The model parameters involving The Council are both an indicator of program effect over time and the effect of location at baseline. Specifically, The Council $\left(\gamma_{01}\right)$ predicts the effect of location (ORV and CJ compared with $\mathrm{CH}$ and IR) at baseline. In other words, this parameter assesses the preexisting differences by location. The parameter Occassion $2 *$ The Council $\left(\gamma_{07}\right)$ assesses location differences in change of level of traditional masculinity over the first 10 -weeks and Occassion $3 *$ The Council $\left(\gamma_{08}\right)$ assesses location differences in change of level of traditional masculinity over the second 10-weeks. Given that the main difference in locations after baseline measurement includes the implementation of The Council, these parameters are assessing the effect of The Council.

The results indicated that after controlling for age, race/ethnicity, and level of ethnic pride, experimental location (control versus experimental sites) predicted differing levels of adherence to traditional masculinity at baseline. Specifically, youth at the experimental locations (ORV and $\mathrm{CJ})$, on average adhered more strongly to traditional masculinity ideology than youth at the control locations ( $\mathrm{CH}$ and IR) by approximately 0.1 units $\left(\gamma_{01}=.096, p=.002\right)$ at baseline. However, participation in 
The Council was not predictive of change in adherence over time when controlling for all other model variables, as was hypothesized.

\section{Hypothesis 2. Age Effect (see Figures 9a\&b)}

H2a. Age will predict adherence to masculinity ideology at baseline.

Hypothesis 2, part a, posited that age will positively predict initial level of adherence to traditional masculinity ideology.

H2b. Age will be a negative predictor of linear change in adherence to traditional masculinity ideology. Hypothesis 2, part b, posited that age will negatively predict changes in level of adherence to traditional masculinity ideology over time.

Hypothesis 2a was supported by the data. As predicted, age was related to initial levels of adherence to traditional masculinity ideology $\left(\gamma_{02}=-.041, p<.001\right)$, however, in the opposite direction than anticipated. Specifically, older participants reported lower initial levels of adherence compared to younger participants. Despite the significant relationship at baseline, age did not have an effect on changes in adherence of traditional masculinity $\left(\gamma_{09}=-.013, p=.486\right)$ over the first 10 -weeks nor over the 20 -weeks $\left(\gamma_{10}=-.019, p=.348\right)$. Thus, hypothesis $2 \mathrm{~b}$ was not supported by the data.

Finally, location (experimental sites vs. control sites) had a moderating effect on the relationship between age and initial levels of masculinity ideology $\left(\gamma_{91}=.044\right.$, $p=.025)$. Specifically, the negative relationship between age and initial level of 
adherence to traditional masculinity ideology is strongest for youth at the control locations and weaker for those at the experimental locations.

Hypothesis 3: Race/Ethnicity Effect (see Figure 10a\&b)

H3a. Race/Ethnic identity will predict adherence to traditional masculinity ideology at baseline.

Hypothesis 3, part a, posited that race/ethnic identity predicts initial level of adherence to traditional masculinity ideology over time.

H3b. Race/Ethnic identity will predict masculinity ideology development (neutral hypothesis).

Hypothesis 3, part b, posited that race/ethnic identity predicts changes in level of adherence to traditional masculinity ideology over time.

The data partially supported hypothesis 3a that participant's race/ethnic identity predicts initial levels of adherence to traditional masculinity ideology. Specifically, after controlling for location (experimental versus control), age, and level of ethnic pride, participants who identified as White had lower initial levels of adherence to traditional masculinity ideology $\left(\gamma_{03}=-.283, p<.001\right)$ compared with African American participants. However, Latino and African American youth were non-significantly different in their initial levels $\left(\gamma_{04}=-.120, p=.258\right)$. Similarly, hypothesis $3 \mathrm{~b}$ was partially supported by the data. In particular, after controlling for location, age, and level of ethnic pride, White participants experienced different trajectories of change in level of adherence to traditional masculinity ideology over the first 10-weeks $\left(\gamma_{11}=.088, p=.025\right)$ and full 20-weeks $\left(\gamma_{21}=.090, p=.028\right)$ 
compared with African American youth. Whereas White adolescent inmates had initially lower levels of adherence to traditional masculinity ideology compared to African American adolescents, their level of adherence increased significantly overtime. Latino participants did not have different trajectories of change in level of adherence to traditional masculinity ideology over the first 10 -weeks $\left(\gamma_{31}=.128, p=\right.$ $.331)$ and full 20 -weeks $\left(\gamma_{41}=.008, p=.957\right)$ compared with African American youth.

\section{Hypothesis 4: Ethnic Pride Effect (see Figure 11a)}

H4a. Ethnic pride will positively predict adherence to masculinity ideology at baseline.

Hypothesis 4, part a, posited that ethnic pride predicts initial level of adherence to traditional masculinity ideology over time. Specifically, it was hypothesized that participants with greater levels of ethnic pride will have higher initial levels of adherence to traditional masculinity ideology.

$H 4 b$. The relationship between ethnic identity and adherence to masculinity ideology over time will be moderated by ethnic pride.

Hypothesis 4, part b, posited that ethnic pride moderates the relationship between ethnic identity and adherence to traditional masculinity ideology over time. Specifically, it was hypothesized that the relationship between ethnic identity and change in adherence to traditional masculinity ideology will be strongest for youth with greatest levels of pride and weakest for youth with low levels of ethnic pride for each race/ethnic category. In comparing between groups, it is expected that both 
White and Latino adolescents will have a smaller effect compared to the African American adolescents.

The data partially supported hypothesis 4a that participant's ethnic pride predicted initial levels of adherence to traditional masculinity ideology, though in the opposite direction than was predicted. After controlling for age, location, and race/ethnic identity, ethnic pride was negatively related to initial levels of adherence $\left(\gamma_{06}=-.032, p=.021\right)$. That is, participants with greater levels of ethnic pride reported lower levels of adherence to traditional masculine ideology. Because participants who identified as African American were most represented in this study, this race/ethnic identity served as the base of the model. Thus, the effect of ethnic pride is an effect for this particular group. On the other hand, this effect was not detected for White $\left(\gamma_{51}=-.017, p=.554\right)$ or Latino $\left(\gamma_{61}=.041, p=.661\right)$ participants.

Hypothesis $4 \mathrm{~b}$ was not supported by the data. Specifically, level of ethnic pride was unrelated to change in adherence to traditional masculinity ideology over the first 10 weeks $\left(\gamma_{71}=-.024, p=.141\right)$ and over the second 10 weeks $\left(\gamma_{81}=-.019, p\right.$ $=.265)$. In addition, level of ethnic pride was unrelated to change in adherence to traditional masculinity for White youth $\left(\gamma_{10.1}=-.040, p=.294 ; \gamma_{10.2}=-.022, p=.556\right)$ and Latino youth $\left(\gamma_{10.3}=-.108, p=.386 ; \gamma_{10.4}=.004, p=.975\right)$.

Hypothesis 5: Prison Effect

H5a. Days in Prison will positively predict initial levels of masculinity ideology. 
Hypothesis 5, part a, posited that the number of days in prison predicts initial level of adherence to traditional masculinity ideology. Specifically, it was hypothesized that participants with greater length of stay in the prison will have higher initial levels of adherence to traditional masculinity ideology.

H5b. Days in Prison will negatively predict change in masculinity ideology.

Hypothesis 5, part b, posited that the number of days in prison predicts change in level of adherence to traditional masculinity ideology. Specifically, it was hypothesized that participants with greater length of stay in the prison will have more stable levels of adherence to traditional masculinity ideology.

As described in the model selection process section above, hypotheses 5a and b were not supported by the data and these parameters were removed from the final model. In sum, length of time in the Ohio Department of Youth Services juvenile justice system had no effect on initial levels of masculinity ideology or trajectories of change in level of adherence over time.

Hypothesis 1. Program Effect (see Figure 8b)

As mentioned above, hypothesis $1 \mathrm{~b}$ utilized a unique model and for that reason is presented here, out of numerical order.

H1b. Greater participation (attendance) in The Council will predict change in adherence. Hypothesis 1, part b, posited that greater participation in The Council will be associated with more negative linear change and that less attendance will be associated with less negative change. That is, the program was expected to challenge stereotypes that endorse traditional masculinity ideology, thus, level of adherence to 
traditional masculinity ideology was expected to decline as a function of program participation. The following model was used to test this hypothesis with participants in the experimental group only $(n=498)$.

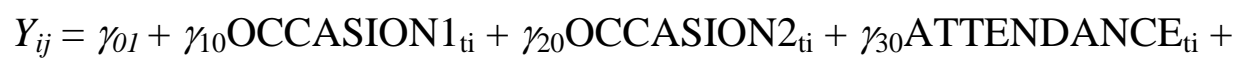

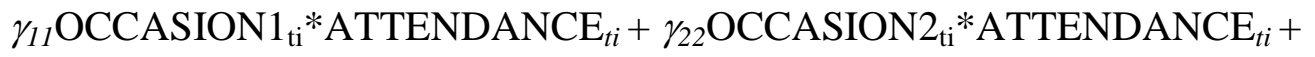
$\gamma_{08} \mathrm{LOC}_{-} \mathrm{CIR} i+e_{t i}$

As was the case with the previous model, in this model, $Y_{i j}$ represents the level of adherence to traditional masculinity ideology for each individual $i$ on survey occasion $j$. The random intercept for person $i$ is $\gamma_{0 i}$, which represents the predicted level of adherence when all model predictors are equal to zero. Thus, the intercept represents the predicted level of adherence for an individual at baseline survey measurement, at Ohio River Valley with zero hours of attendance. The first survey occasion coefficient in the model represents change from pre- to post-survey and the second survey occasion coefficient represents change from pre- to final-survey occasion. The attendance coefficient represents the amount of attendance in The Council in total hours over the course of 20 -weeks. Additionally, the interaction terms of the model were created by multiplying the survey occasion variables to the variable representing attendance. These interaction terms allowed me to assess the dosage effect of The Council on trajectory of change. Finally, the random error of the model is represented with $e_{t i}$.

The results indicated that the average level of adherence to traditional masculinity ideology at baseline for participants at Ohio River Valley with zero 
hours of attendance in The Council was $2.27\left(\gamma_{01}=2.27, p<.001\right)$. Additionally, although descriptively the level of adherence increased linearly by an average of approximately .05 units $\left(\gamma_{10}=.045, p=.138\right)$ for the first 10 weeks when controlling for attendance in The Council and participant's location within the experimental sites, this change was not statistically significant. However, change from baseline to the final survey measurement was statistically significant and level of adherence increased linearly by an average of .09 units $\left(\gamma_{20}=.092, p=.009\right)$ controlling for the other variables in the model. Interestingly, attendance in The Council was positively related to level of adherence at baseline $\left(\gamma_{30}=.004, p<.001\right)$, such that individuals who attended more hours of The Council throughout the course of 20-weeks had higher initial levels of adherence to traditional masculinity ideology than those with less attendance. Additionally, attendance in The Council was significantly related to change in level of adherence for the first 10-weeks $\left(\gamma_{11}=-.004, p=.023\right)$ and for the entire 20 -weeks $\left(\gamma_{22}=-.005, p=.012\right)$. Specifically, after controlling for all other model variables, youth with average attendance in The Council (10-week $M=14.26$, $S D=6.11 ; 20$-week $M=13.21 ; S D=5.4)$ increased by less than one twentieth of a unit on the 5-point Likert Adolescent Masculinity Ideology in Relationship Scale over the first ten weeks $(10$-weeks $=.045)$ and the second ten weeks $(20$ weeks $=$ .095). However, youth with attendance one standard deviation below the mean increased at a greater rate $(10$-weeks $=.069 ; 20$ weeks $=.122)$ and those with attendance one standard deviation above the mean increased by a lesser rate (10weeks $=.020 ; 20$ weeks $=.067)$. That is, youth who attended $90 \%$ or more sessions 
(approximately 1 standard deviation above the mean) increased at a rate approximately 2-3 times (10-weeks $=3.45$ times; 20 -weeks $=1.82$ times $)$ that of youth who attended $40 \%$ of the sessions (approximately 1 standard deviation below the mean). Thus, as was predicted, greater attendance in The Council predicted lower levels of adherence to masculinity ideology compared to lower levels of attendance. Finally, the intercept variance of level of adherence to traditional masculinity ideology remained significant, $\tau_{00}=.072, p<.001$, indicating that baseline levels of adherence varied significantly across participants after controlling for experimental location and attendance in The Council.

Research Question 2: What do youth who participate in The Council learn about being male?

Research question 2 was assessed using "thematic analysis", a qualitative method for identifying, analyzing, describing, and reporting patterns within data (Braun \& Clarke, 2006; Spencer, Ritchie, O’Connor, 2003). I used Braun and Clarke's definitions of terms to describe the data that were analyzed using this methodology. The data corpus, all the data collected for this research project, included the participant paper-and-pencil responses and ODYS records data. For the current project, the data corpus included responses from 1,248 possible participants for four items at two different survey measurement occasions (after the first and second 10-weeks of The Council). Thus, the data corpus is equal to 9,984 units of text. The data set included all of the open-ended qualitative paper-and-pencil responses that may be analyzed in addressing this particular research question. To be 
clear, only participants at Ohio River Valley and Circleville, the experimental locations, at post- and follow-up survey occasions were given the opportunity to respond to these open-ended questions. Thus, the data were reduced to the 498 participants at the experimental locations. Multiplying this number by the number of items (4) and survey measurement occasions that supplied these questions (2) yields a product of 3,984 possible data units. The data item refers to each response to a qualitative open-ended question.

Of the four open-ended items, only one of the items directly assessed the construct of masculinity (S9). Thus, all responses to the item S9, "What have you learned about being male?", were included in the data items. Of the 498 possible responses to this item at two different time points, 392 (39\% of the total possible 996) responses were present. In addition, all responses to the other three items, S8. “What have you learned in Boys \& Young Men's Council?”, S10. “What have you liked and/or disliked about Boys \& Young Men's Council?”, and S11. "Have you changed in any way after being a part of Boys \& Young Men's Council? " that describe something related to being male were included. For these three items, I first filtered for those with responses, as was done with item S9. For item S8, there were 408 (41\% of the possible 996) responses. For item S10, there were 372 (37\% of the possible 996) responses. For item S11, there were 385 (39\% of the possible 996) responses. Thus, the total data items were 408 (S8), 392 (S9), 372 (S10), and 385 (S11), which summed to 1,557. Finally, the data extract included all coded data that were extracted from the 1,557 data items for the purposes of answering the research 
question (RQ2) described above. I subdivided the section to follow into five parts that parallel the five phases of analyses described by Braun and Clarke, (1) Gaining familiarity with the data; (2) Generating initial codes; (3) Searching for themes; (4) Reviewing themes; (5) Defining and naming themes; (6) Producing the report. The purpose of the thematic analysis of the qualitative, open-ended survey responses was to inform the predominantly quantitative investigation into masculinity ideology among adolescent inmates over time by describing what youth learn about being male. Specifically, the goal of the analysis was to assess what the participants say about being male in an open-ended format. In particular, the analyses aimed to understand how youth participating in The Council describe what it is like to be male and what they learned about being male as a result of their involvement in the program. The open-ended responses have the possibility of providing a deeper understanding of hypotheses that were supported by the data, as well as providing insight as to why hypotheses were not supported by the data. In addition, the open-ended responses may highlight areas of masculinity and what youth describe as important indicators of being male that are not captured by the quantitative measure. Intertwined throughout each of the phases of thematic analysis, I engaged in a process of analytic memo writing. Analytic memos (also known as researcher memos) is a way in which the researcher engages in conversation with her/his self about the data and the research process that is systematic and ongoing (Ely, Anzul, Friedman, Garner, \& McCormack Steinmetz, 1991). My analytic memos described what I learned and the insight that was gained with each iterative 
phase of the research. I also described how information gained at each phase informed the subsequent steps in the research process (Ely et al.). In the paragraphs to follow I describe the steps I took to fulfill each of these phases of inquiry and analysis and how engaged in an iterative process of analytic memo-making. Finally, I describe the results of the qualitative analysis throughout the subsections to follow.

Phase 1: Gaining familiarity with the data: In the first phase, I managed the data by first identifying the data set and data items. I described above how the data set and data items were determined. From the resulting data set and items, I further reduced the data for responses to items S8 and S10-S11, resulting in the final data extract. Specifically, given the goal of this particular analysis stated above, I identified and carefully selected only responses that directly or indirectly relate to the focus of what youth learned about being male. In the three paragraphs to follow, I will describe the process that was taken to select for responses to each of the three items. As a reminder, given the nature of the question, "What have you learned about being male?", all responses for item S9 were selected for the analyses.

To begin, I read through the responses to item S8 twice to familiarize myself with the data. On my third pass, I categorized the responses into relevant and irrelevant (to the research question) groups. In this pass I made an attempt to be inclusive and liberal in my coding, such that only responses that were clearly irrelevant or too generic (e.g., "No", "Nothing new", "A lot", “A lot of important things", “The same shit”) were coded as such. All responses with more substance were included on this pass. In total, 342 (34\% of the 996) responses were included in 
this pass. In my fourth read, I took a more conservative approach and coded responses as irrelevant when they were substantive, but unrelated directly to the concept of masculinity ideology (e.g., "That's its not good to do crime", "I've learned that if I stop doing crimes, a lot of good things will happen for me.", "When we did the maizes", "Don't come 2 jail.”, "that these young punks is fuc niggas", "Not a dam thing so stop giving us the bull-sh-t were not no lab rats bitches"). In this final pass, 287 (29\% of the total 996) responses were coded as relevant and make up the final data extract for this item.

Second, I followed the same procedures as is described above for responses to item S10. After two full initial reads of the responses, I categorized the responses into relevant and irrelevant (to the research question) groups. In this pass I made an attempt to be inclusive and liberal in my coding, such that only responses that were clearly irrelevant or too generic (e.g., "Nothing 4 real”, “Activitees", "How long it is", "I liked everything", "Candy") were coded as such. All responses with more substance were included on this pass. In total, 225 (23\% of the 996) responses were included in this pass.

In my fourth read, I took a more conservative approach and coded responses as irrelevant when they were substantive, but unrelated directly to the concept of masculinity ideology (e.g., "It's ok to me it's something to do to keep me busy.", "I liked that it looked good on my report.", "I disliked that the group lasted too long but it was worth it.", “Take up too much free time!”). In this final pass, 111 (11\% of the 
total 996) responses were coded as relevant and make up the final data extract for this item.

Finally, I applied the same procedures as is described above for responses to item S11. After two full initial reads of the responses, I categorized the responses into relevant and irrelevant (to the research question) groups. In this pass I made an attempt to be inclusive and liberal in my coding, such that only responses that were clearly irrelevant or too generic (e.g., "No", "Yes", “A little”, “can’t say right now.", "I always bee the same.") were coded as such. All responses with more substance were included on this pass. In total, 191 (19\% of the 996) responses were included in this pass. In my fourth read, I took a more conservative approach and coded responses as irrelevant when they were substantive, but unrelated directly to the concept of masculinity ideology, overly general, or not clearly a description of change (e.g., "My crime. stuff.", "Yes. I've matured more", "I can see clearer", "Yes a little but I know the tools Im just not using them at this time", "No but I took a lot of notes on how to change.”). In this final pass, 86 (9\% of the total 996$)$ responses were coded as relevant and make up the final data extract for this item.

The results of the process of data extraction are described in Table 14. In sum, 897 data responses $(23 \%$ of the total 3,984$)$ are included in the data extract for all four items.

As transparency is foundational to this thematic coding process, I will briefly address this concept before introducing the following phases of analyses. To begin, because of the thin responses provided by the youth, making inferences on the data 
minimal, I was the sole coder of the data. For this reason, I made a point to describe each theme in detail providing illustrative responses throughout. For example, in response to the question "What did you learn about being male?" one youth responded 'Most males are alike in a certain way' and another male responded with 'That its okay to express yur feeling”. With ease these responses highlight two distinct male concepts - (1) Male relationships; and (2) Emotional expression. However, a more complicated response, such as, 'That that tings most of us worry about like power \& control really don't mean much. And most of us think the same' touches on both the concepts described above. The coding process was done in such a way that one data response may fall under multiple themes, or in other words, the data are not mutually exclusive. Thus, because the data are thin and may be coded under multiple themes, the coding decisions were relatively simple and straight forward.

Phase 2: Generating initial codes: In the second phase of analysis, I coded for features of the data that inform the research question posed. To begin, I separated the responses into two groups. The first group contained all responses to the questions not directly pertaining to masculinity ideology (S8, S10-11). The second group contained all responses to question S9. I read once through all of the responses that were selected in the first phase of analysis and all responses to S9. On the second pass I made notes next to each data response. At the conclusion of notating each response set (item) within the first group, I took memo-notes. Once each data extract of the first group was noted with an initial code and all memo-notes were 
made, I read through all responses of the second group. Again I made notes next to each response on the second pass. Upon completion of the initial coding, I constructed a list of all unique codes in excel (e.g., Conceptual Framework or Index; Ritchie, Spencer, \& O’Connor, 2003), to prepare for the third phase. Responses to item S8 generated 41 unique codes, responses to item S9 generated 50 unique codes, responses to item S10 generated 26 unique codes, and responses to S11 generated 45 unique codes. In total, there were 114 unique codes.

Phase 3: Searching for themes: Next, I began the process of generating broader level themes in which the codes could be synthesized. Again, the purpose of this analysis was to inform the quantitative analyses by describing what youth say about being male and what they have learned about being male. In addition to providing support and insight for interpreting the quantitative findings, the responses may highlight important aspects of being male that are not captured by the quantitative measure. Thus, at this stage I went back to the literature, in particular Chu and colleagues (2005) operationalization of masculinity ideology. The general themes of the Adolescent Masculinity Ideology in Relationship Scale are (1) emotional stoicism, (2) heterosexual dominance, sexual "drive”, (3) physical toughness, and (4) competitive and ambition (J. Chu, personal communication, February 2, 2008). With these themes in mind, I organized the codes using mindmaps, placing similar codes in the same physical space. I structured the initial groupings based on the four themes listed above. Because the codes did not neatly fall under those four themes, I added a fifth theme, "other" to capture all other 
responses. In the first pass, it was clear to me that these themes were relevant to the responses provided by the participants in this study. After organizing the codes into the five themes, I read through each grouping and wrote out three to six the subthemes of each theme. The reason behind this step was to make more distinct groupings of the data, rather than using only five broad categorizations. For example, under the broad theme 'Emotional Stoicism' responses included both reflections of emotional stoicism or inexpression as well as emotional expression. Therefore, it seemed important to make a distinction within this theme. Ultimately, each code (data response) was nested under a specific sub-theme, which was further nested within a theme. In the end, there were 5 themes and 19 sub-themes (see Table 15).

Phase 4: Reviewing themes: During phase 4, I made additional organizational decisions about the themes. Specifically, I broke out some of the themes identified in Phase 3 into two or more separate themes. Additionally, I lumped some of the subthemes together. The 'Other' theme was deleted and made into more specific themes. In the end, there were eight themes with one to three sub-themes nested within them (see Table 16). The eight themes were (1) Emotional stoicism; (2) Heterosexual Dominance; (3) Physical Toughness; (4) Competitive \& ambition; (5) The Councilgroup specific; (6) "Man Up”; (7) New perspective; and (8) Relational.

The rationale behind selecting these eight themes was made based on the goal of capturing the messages of the qualitative data and categorizing the data in groups, in which the data internal to the group are similar but distinct from data in other groups. The structure of these themes and sub-themes were visualized in a thematic 
map. Once the map was set, I went back to the data to determine if the map fit the data well. After noticing that the changes I wanted to make were minor, but that, on the whole, the data fit the map well, I made only a couple minor tweaks to the thematic map (see Figure 3).

Phase 5: Defining and naming themes: During the fifth phase of analysis, I defined each theme by identifying and naming the theme carefully such that the name and definition captured the nature of the data within the theme. Specifically, for each individual theme I named the theme and wrote a concise definition of the theme. Moreover, I described the story that each theme tells in relation to research question 2. During this phase of analysis, I grouped sub-themes under main themes such that the themes were further refined to best capture the data. The titles, definitions, and stories of each theme are described in Phase 6 below.

Upon receiving feedback from my dissertation committee during my predefense meeting, the naming of the themes were revisited. In particular, several themes encompass paradoxical subthemes, such as emotional expression and inexpression. However, the theme names do not adequately capture this paradox. Thus, it was suggested that I rename the themes using the actual responses from the youth, as was done for the theme "Man Up", such that they capture the paradox of the underlying subthemes and are written in the participant's own words. I struggled to find short, catch-phrases in the youth's responses that could be used for naming some of the themes. Therefore, I instead created a major theme name that captures the subtheme paradox and illustrated these themes with quotes from the youth for 
these themes. For others, I was able to use a phrase quote from the youth. The final naming of the eight identified themes are displayed in a final thematic map (Figure 4).

Phase 6: Producing the report: The last phase of qualitative analysis is where I report the findings of the thematic analysis with detail and example quotes, such that the process of analysis is fully transparent, coherent, and logical. The number and percentage of data responses that were coded into each of the eight themes are presented in Table 17. This phase was presented throughout the reporting of the preceding phases. Additionally, in the paragraphs to follow I briefly describe the story that is represented by each theme and how it helps to answer the guiding research question. The purpose of this description was to provide a solid understanding of the process and the thematic content of the qualitative data. In the section to follow, Mixed Method Analysis, I conclude the phase 6 description by describing how the quantitative findings aid in the evaluation and interpretation of the quantitative findings. Specifically, I describe where there is overlap in the qualitative themes and the quantitative findings and where the qualitative themes provide a unique understanding of how youth describe what they learn about being male that was not captured by the quantitative analysis. Additionally, I describe how the open ended responses allow for a deeper exploration of concepts that are captured by the quantitative measure.

Emotional awareness: “Male's have feelings to[o]' vs. '...cant let nobody see you cry' is a theme that encompasses responses regarding lack of emotional 
expression or the expectation to be emotionally stoic as well as responses regarding emotional expression. This theme captures feelings and emotions other than anger that are not associated with traditional masculine ideals, such as sadness, vulnerability, and fear. Several responses to each of the four open-ended questions describe participants' newfound awareness or openness to emotional expression, or expression of specific or general feelings. For example, responses that were coded under this theme and under the sub-theme of emotional expression were:

"I learned how to express myself." (S8)

“That its okay to express my feeling." (S9)

"Male's have feelings to[o]." (S9)

"I learn that you ain't gotta hide yo feelings just because you're a male it's ok to express your feelings". (S9)

“That expressing your feelin don't mean you're a coward.” (S9)

"What do I like about boys counciling? The way we spoke freely. What did I dislike? I disliked nothing." (S10)

"I changed as an individual by learning to express myself to others and not hold back anything." (S11)

On the other hand, some youth described an expectation that they are not free to express emotions or a frustration with others for either expressing their feelings and emotions or not expressing their feelings and emotions. For example, responses that were coded under this theme and under the sub-theme of emotional inexpression were: 
"How to keep my feelings to my self." (S8)

"That it's ok to cry but you just cant let nobody see you cry." (S9)

"Not to be ashamed to be a male. Males are more likely to keep their feeling to theirselfs. Its harder for males to express themselves." (S9)

“That in many cultures boys are made into men by not having emotional outburst in other words youth can't be emotional.” (S9)

"People cry to[o] much for me and I liked the activities we did." (S10)

"The ones who is scared to speak there mind." (S10)

It should be noted that the sub-theme of emotional stoicism was much less frequently used to categorize the responses than its opposing sub-theme regarding emotional expression. The categorization of this theme and the opposing sub-themes help inform the research questions of this dissertation in several ways. To begin, the responses categorized as emotionally stoic were diverse. Whereas a small percentage of the responses fell on the dimension of emotional stoicism (one sub-theme), which parallels traditional masculine ideals, most responses were on the dimension of emotional expression (the other sub-theme) that opposes traditional masculine ideals. Two major outcomes are evident. First, youth were diverse in their responses regarding emotional expression. Second, most youths' responses establish that change occurred determined by time in The Council, such that youth were more inclined to express emotion. Finally, several responses coded under the sub-theme of emotional expression described an awareness regarding the breakdown of the expectation of traditional masculinity regarding emotional stoicism. Specifically, 
youth explain emotional expression as a common and acceptable behavior that does not and/or should not affect their status as a young man. In this way, youth explicitly combat the expectation that men are to be emotionally stoic. As is displayed in the thematic map (see Figure 3), this sub-theme is related to the theme "Man Up" that directly captures some of the traditional male expectations and other ways in which youth describe a breakdown of these expectations.

Gender Awareness: "man do what they want..." vs. "Respect women..." is a theme that encompasses responses regarding both male dominance or male and female differences, as well as responses regarding gender equality. This theme captures ideas surrounding gender and sexuality, including male attraction of females, male dominance over females, neutral but described differences between male and females, as well as some responses that captured ideas surrounding gender equality. Several responses described a general and neutral idea that males are different from females or that males were different and more superior to females. For example, responses that were coded under this theme and under the sub-theme of masculine dominance were:

"We're the superior gender" (S9)

"We are the best sex." (S9)

"That I am most dominate." (S9)

"I LEARNED THAT WE HAVE STRONGER INSTINCTS AND QUALITIES THEN FEMALE.”(S9) 
"I liked learning all the things about how the world looks at males, I disliked that most women thing men are arrogant." (S10)

"I got what A Girl wants yea buddy." (S9)

“That female are More Atractive aNd other Males are Not of iNterst." (S9)

"man do what they want and la[d]ys do what they can." (S9)

"That were different from female" (S9)

"I love being male and male's have a little more contRol than women and male's have to Be moRe Role model's And safe." (S9)

On the other hand, some youth described learning to respect women and describe masculinity as equal and/or unprivileged. For example, responses that were coded under this theme and under the sub-theme of gender equality were:

"That Being a male doesnt mean you can do what you want to do In life." (S9)

"Respect women more and their thoughts" (S9)

"male are the Best thing in life." (S9)

"Someway I think diffent I respect females more and I look at them diffent." (S11)

The categorization of this theme and the somewhat opposing sub-themes help inform the research questions of this dissertation in several ways. To begin, several youth describe learning about differences in males and females. In my own observations of The Council facilitator training groups (Seattle YMCA, December, 2008) biological differences between men and women were a focus of the teachings 
for some of the day. Thus, this finding is entirely consistent with the messages of the program and the facilitators. Interestingly, some of these responses highlight differences, by placing males as superior to or better than females. Additionally, some youth describe heterosexual interest in females. It is unclear in these responses whether this is regarded as an expectation, that of males being attracted to females. Finally, a small sample of youth described respecting females more as the result of The Council. These responses counter some of the male dominant responses and highlight the diversity of responses regarding heterosexual or masculine dominance. In other words, although most responses that were categorized in this theme were true to its name, some opposed it. Thus, youth appear to be taking away different types of messages regarding gender differences and power.

Physical Awareness: “you don't have to be a punk if you walk away from a fight" vs “...we are sopost to be strong” is a theme that encompasses two subthemes; (1) avoidance of fights and conflict, and (2) the expectation to be tough and strong. For each of these sub-themes, there are opposing ideas expressed. For example, responses categorized under the first sub-theme both described ideas of avoiding these situations, but also learning to engage in these behaviors. The second sub-theme also captured opposing responses. For example, some youth describe an expectation that they are supposed to be tough and strong, whereas others describe a recognition that this expectation does not hold. Often times when this expectation is countered, the youth make a connection of this expectation with their status. 
Specifically, they describe how they do not have to be tough and strong to be cool or gain status. Examples of responses that fall under the first sub-theme are as follows:

"How to walk away from a fight. To thank about my family more. To stop doing crime." (S8)

"How to control my self Better, Angerwise." (S8)

"About taking in something and how to handle stress and conflict." (S8)

"I have learned that you dont have to be a punk if you walk away from a fight." (S9)

"you dont have to always fight people." (S9)

"How to fight." (S9)

"I liked that we worked together \& no fighting was involved." (S10)

"yes stop fighting and geting in trouble." (S11)

"I have controlled my anger alot more, and have learned how to avoid or stay away from fights.” (S11)

Examples of the second sub-theme are as follows:

“That I don't have to prove im tough or anything just to get respect Just be myself." (S8)

"That I don't have to act tough to be a man." (S9)

“That we are sopost to be strong." (S9)

"That we Don't alway have to Be Tough." (S9)

"It's okay to cry and I don't hafe to be a tough guy all the time." (S9)

"You have to be strong regardless of the situation." (S9) 
"I liked people in group that speak openly. I dislike people scared or too tough.” (S10)

"Yes because i learned That you dont always have to be tough about everything." (S11)

The categorization of this theme, the two sub-themes, and their nested opposing viewpoints help to inform the research questions of this dissertation in several ways. To begin, several youth describe learning tools or strategies to avoid conflict and fights. Only a handful of youth, on the other hand, describe instances in which conflict or fights took place or learning behaviors that may be categorized as physically aggressive. What may be taken from this is that youth tended to learn avoidance behaviors regarding physical violence and aggression. The second subtheme captured a very common response regarding the realization that one does not need to act tough to gain status. This dimension of the sub-theme directly counters the expectation of traditional masculinity that men are expected to be tough and strong in all situations. Far fewer youth respond in ways that are categorized on the other dimension of this sub-theme that are in line with traditional masculine ideals and expectations. To sum, most youth appear to be gaining awareness that acting tough is not linked to status. Thus, adherence to this aspect of traditional masculinity ideology appears to be decreasing for some youth participating in The Council.

Leadership, Status, \& Self-Efficacy: “... be a role model!” vs. “doing negative stuff does not make you any cooler than anybody else" vs. "I fEE like I can go far in life" is a theme that encompasses three sub-themes: (1) leadership; (2) 
status; and (3) self-efficacy. Broadly, the theme captures responses regarding the expectation or ability to take leadership roles and be strong role models for other boys or youth. This sub-theme was described from a positive perspective and is distinct from the sub-theme 'responsibility' that falls under the theme 'Man Up'. Additionally, this theme encompasses responses regarding positive status gained through programming or realizations revolving status that results from behavior. Finally, this theme includes responses that are self-efficacious in nature or that are future oriented. Examples of responses that fall under this theme and the designated sub-themes are as follows:

\section{Leadership}

"To be a better leader iN More positive ways" (S8)

"How to be a role model!" (S8)

“ABout Being Responsible take leader ship do whats Right start acting like a young man don't follow Be a leader." (S8)

"To be a rolemodle". (S9)

"That it's my role in life to be strong and be a good role modle for younger kids." (S9)

\section{Status sub-theme}

"That doing negative stuff does not make you any cooler than anybody else."

"yes i Have changed my Behavior Towards my peeps NOTE: i obtain my level 4 (Haven't had it since i've Been in Dys: X/X/XX)" (S11) 


\section{Self-efficacy}

"How to be succesfull and make it in life when I get out of an institution."

"I learned to start preparing my goals for when I leave to go home. Also to broaden my horizon for doing positive things while living in the community." (S8)

“yes I Have chaNged ANd learNed How to Make New choices" (S11)

"Yes. I have changed a lot. I fEE likE I can go far in lifE." (S11)

The categorization of this theme and three sub-themes help to inform the research questions of this dissertation in several ways. In general, this theme highlights the expectations the youth have for themselves regarding their future, their status, and leadership. For the most part, responses categorized under this theme were positive for youth who describe wanting to be a good role model and learning how to become strong leaders. Additionally, youth seem to reflect positively on their future and are making goals for a positive life outside of ODYS. These responses infer a positive gain that is made based on participation in The Council. Additionally, these responses may be indicative of increases in dimensions of traditional masculinity ideology having to do with status and success.

"Boys Council" is a theme that was used to capture all responses that were specific to the participation in The Council, but not directly answering the research question posed. This theme is important as it may indirectly inform the research question regarding how youth changed over time. The responses to follow were categorized into this theme: 
“I LIKED THAT it LookED gooD ON mY REPORT.” (S10)

"I disliked the leader of my boy's council." (S10)

"I hate J.C.O's At O.R.V. They Are Racist." (S10)

"It seems like there always Judgeing You." (S10)

"We dont have that much stuff to talk about and the fact that its boys council not mans council because I am 20yrs old I know about almost everything we talk about my unit is $18+$ older." (S10).

"That we have to read in that you have some boysin the group that like to say something bad Bout you." (S10)

These responses may help explain why some youth seem to be changing in different ways from others. As is shown in the quotes above, some youth seem to be enjoying their participation in the program, whereas others are not. Additionally, youth give a variety of different reasons for why they like or dislike participating in the program. For example, some youth describe youth or facilitators in their group as being untrustworthy or disrespectful. The nature of these groups may not be conducive to positive change in the same way as the groups that are described positively. Finally, a handful of youth describe The Council curriculum or aspects of their group as immature. This is important as it may help inform some of the quantitative findings regarding the effect of age on youth's trajectories of change.

"Man Up" is a theme that captures responses that are specific to general expectations of traditional masculinity ideology. The wording of this theme was selected based on the language of the youth. Several youth wrote responses regarding 
the expectation or learning how to "man up" or "men up" in a general sense. In addition, youth describe general roles of men, such as simply stating 'roles of men'. These responses, if written out of context of any other theme, were grouped into this theme. Others describe certain expectations that are associated with traditional masculine ideals, most notably that of 'responsibility'. On the other hand, this theme also captures responses that described an awareness of a breakdown of the expectations of traditional masculinity. Sometimes responses under this sub-theme described an alternative more flexible masculinity. Other times these responses described an awareness of status being unassociated with the expectation. Some of the responses that were categorized under this theme and the "man up" sub-theme are illustrated below.

"Roles oF Men.” (S8)

"How to be a man and the meaning of being man" (S8)

"how to man up and work together wit other peers." (S8)

"I learned What it Really take to become a man the real way not the "Street Punk" way.” (S8)

"How to man up" (S9)

“The man Code." (S9)

"That there are responsiblities that I have to take care of to show im a real male." (S9)

"I have changed by working torse into becomin a real positive man." (S11) 
"you will learn how to handle your rosponsibilities and present yourself as a young man." (S8)

"To be positive stay focus Responsibility." (S8)

Some of the responses that were categorized under the "man up" theme and the "breakdown of traditional masculine stereotypes" sub-theme are as follows. The first quote is illustrative of both sub-themes.

"That it's okey to get help." (S8)

"How to bE A bEttER young MAN iN lifE ANd it hElpEd ME WoRk hARdEr ON MY goAls so I bE whAt I cAN bE.” (S8)

"I Learned that in Boy's Conuncil you can change your Negative ways to positive ways and still be Accepted.” (S8)

"How to man up in take the concequenses I Have to serve in I also Learned How to Be a Better Person in a Positive Way!" (S9)

"there are alot of stereo-types." (S9)

The categorization of this theme helps inform the research questions of this dissertation in several ways. Most notably, this theme directly captures the youths' responses regarding traditional masculinity ideology — their personal reflections and their reflections on what was learned from their participation in The Council. Often the expectation that was described was very general, for example, to 'man up' or 'be a man'. However, several youth described specific expectations of taking responsibility. It should be noted that responses including the word 'responsibility' was seen in two distinct contexts. Responsibility that was coded under this theme 
was general in nature was in regard to the expectation or learning of taking responsibility for one's actions. This is distinct from the responsibility-related responses that were categorized under the 'relational' theme and the 'family roles' sub-theme, which is described in greater detail below. Though these responses were relatively general, the data under this code are indicative of increases in awareness of or adherence to the expectations that are associated with traditional masculinity. Sometimes this awareness seemed to be an awareness of the breakdown of the expectation associated with being a man. However, responses coded under this subtheme were much more infrequent than those coded under the "man up" subtheme.

New Perspective: “...keep my head up” \& “...my action can hurt other people" is a theme that captures responses regarding new perspectives gained through the participation in The Council, personal growth, and awareness of the consequences of negative actions. This theme is related to the sub-theme, 'breakdown of traditional masculine stereotypes', described above. The difference between the two is that those under the 'man up' theme were specifically related to masculinity. The new perspective categorized more general responses regarding personal growth and personal awareness. Some of the responses that were categorized under this theme and the 'personal growth' sub-theme are as follows:

"To keep my head up when times are good and bad. To stay away from negitivitiy. To bascially Learn how to suceed in life." (S8)

"I am grown up a lot more.” (S9)

"I've grown up." (S11) 
"I changed my attitude In the way I act Before I entered group." (S11)

"yes I changed I became more wiser." (S11)

"Yes I feel more mature in this group." (S11)

The second sub-theme captured responses regarding learning and association between action and consequences and learning to take responsibility for such actions. Some of the responses that were categorized under this sub-theme are as follows.

"I learned to think before I act, and do domb things." (S11)

"I have learned how to be a young man and I have learned that my action can hurt other people.” (S8)

"I learned that discrimination can be hurtful." (S8)

The responses under this theme help explain some of the change that the youth are experiencing. These responses are indicative of the youth's perspectives on how they have changed in a positive way. The sub-theme 'awareness of the consequences of negative actions' may be particularly informative for the questions regarding the influence of prison on changes in masculinity ideology. This linkage will be explored further in the Discussion chapter of this dissertation.

Relational: “...Healthy Relationships”, “....A strong man ... can do A lot to help people”, “...man of the house”, “...Respect” is a theme that captures responses regarding relationship building, understanding commonalities with other young men, and family specific roles and expectations or relations. A large percentage of data responses were coded into the relational theme $(n=225 ; 25.1 \%)$. Specifically, this theme was made up of 4 sub-themes: (1) relational/commonality with others; (2) 
problem solving skills/helping behavior; (3) family roles; and (4) respect. Illustrative quotes for each of these sub-themes are provided below.

The sub-theme relational/commonality with others captures responses regarding relationships, teamwork, and an understanding of shared commonality with other people, in particular, with other males. The first several quotes illustrate the relationship and teamwork aspects of this sub-theme. The last couple of quotes illustrate the shared commonality that youth became aware of throughout the program.

'Being a man. Being Honest. Healthy Relationships.' (S9)

How to work together with other people.” (S8)

"I learned How to coumutiescate with orther men better." (S8)

"That alot of people have alot of thiNgs in commoN aNd this group we have teach aNd help you how to be a maN."” (S8)

"That me and a lot of other youth all have many things in common." (S8)

The sub-theme problem solving skills/helping behaviors captures responses regarding the skills youth learned in the program regarding how to work through their own problems and how to assist and help others in need.

"To help Pepole Out and Longs ass I do the Right thing thats what matter." (S8)

"I learned that we all have alot or simalarties. How the press can make someone look what they want them to look. And that everyone needs help no one talks alone." (S8) 
"Im A strong man and can do Alot to help people." (S9)

"That, If you TAlk ABouT your ProBlems you can, solve Them."

"I leaRned how to deal with situations Be cool with pRoblems." (S11)

"Yes I can say that because it made me reflex on certain issues I deal with and how hearing someone else talk about helps me to work these issue out." (S11)

The Family Roles sub-theme captures responses regarding family relations and is illustrated with the first two quotes below. Additionally, this sub-theme captures responses regarding a male's expected role as provider, protector, and head of household.

"That you Are NeveR Too old to Give mom and Dad hugs and kisses in public.” (S8)

"Yes, I have gotton ahold of my little sibleng and let them know How I feel. let them know their big Brother wil always be around." (S11)

"I learn that a male has to protect his family and provide." (S9)

"that you have to take care yo Family and help them out go out and get a Job"

"the man of the house." (S9)

"I got TO keep The family safe" (S9)

Finally, the last sub-theme respect captured responses that described the importance of being respected and showing respect for others. The word "respect" was very common in responses to all four questions.

“To Respect others and to help others nomatter who they are." (S8) 
"how to cAre about someone AND Show Respect." (S8)

"I have learned How to Show Respect." (S8)

"That if you feel somethings bad going to happen, be smart and dont go.

Respect and treat the next person how you want to be treated. Don't be scared to tell a person (NO) you don't want to do something." (S8)

The responses under this theme help inform the research questions of this dissertation in several ways. For example, this theme counters the male expectation of independence (e.g., The Sturdy Oak) and in turn describes the importance of relationships. Several youth specifically describe relating to other males or other males in their Council group.

\section{Multifaceted Data}

The eight themes presented above, describe participants' responses to openended questions regarding what they learned about being male in The Council. Though the themes are distinct, several nested sub-themes are related to sub-themes nested within other themes. Moreover, several responses, some of which are presented above, may be coded under multiple themes given the multiple foci of the response. For example, in response to the question S9, "What have you learned about being male?", three participants responded in the following three ways:

"That you don't have to look tough to get a good girlfriend or good job." (S9) "BEiNg a MAlE you doN't AlwAys hAvE to put oN A show, show No fEEliNgs, ANd plAy tough.” (S9) 
“1). I can loose my tough image and that it doesnt make me any more manly by doing so. 2). I have a lot more in coomon w/ other men my age.” (S9)

Each of these responses were coded under more than one theme.

\section{Mixed-Method Analysis}

This dissertation uses a Qualitative Follow-Up Sequence Design (Morgan, 1998) for the purpose of evaluating and interpreting the results of the primarily quantitative study. In this final section of the Results Chapter, I use the qualitative findings to support or inform the quantitatively assessed hypotheses. I first present the hypothesis and result of the assessment of the hypothesis. Next, I discuss how the qualitative analyses might help inform the quantitative findings. When possible, I provide examples from the responses provided by the youth to illustrate the conversation between the quantitative and qualitative findings.

Hypothesis 1. Program Effect

Hla: Participation in The Council will predict change in adherence to traditional masculinity ideology.

H1b. Greater participation (attendance) in The Council will predict change in adherence.

Quantitative results indicated that after controlling for all other model variables, youth at the experimental facilities (Circleville and Ohio River Valley) initially adhered more strongly to traditional masculine ideals compared to those at the control facilities (Cuyahoga Hills and Indian River). However, whether or not youth participated in The Council did not affect the trajectory of change in level of 
adherence over time. Interestingly, hypothesis $1 \mathrm{~b}$ was supported by the data, which found that youth at the experimental facilities with greater levels of attendance increased their level of adherence at a lesser rate than those with less attendance. To summarize, youth in the experimental facilities initially adhered more strongly to traditional masculine ideals at baseline, but changed over time at a non-different rate as those youth at the control facilities. However, the amount of The Council sessions a youth at the experimental facilities attended influenced the rate of change over time, such that youth with greater attendance increased their level of adherence to traditional masculinity ideology at a lesser rate than those with less attendance. Thus, although the dichotomous predictor of The Council participation (no participation vs. some participation) did not predict change, the dosage in which a youth participated was predictive of change.

Though the qualitative data was unconnected to the variables that indicate the facility the youth inhabited or the level of attendance in The Council, only youth at the experimental facilities completed this portion of the survey. Moreover, several responses directly describe what the youth learned about being male due to their participation in the program. Therefore, some of the themes (e.g., 'Boys Council', 'Emotional Awareness'), presented above, including some individual responses that were filtered out of the qualitative analyses during early phases due to its lack of relevance to the research question, may enhance our understanding as to why hypothesis 1a (program effect) was not supported by the data, whereas hypothesis $1 \mathrm{~b}$ (dosage effect) was supported. 
To begin, although Circleville and Ohio River Valley implemented The Council at their facilities, it was discovered through qualitative analyses that not all youth participated in the groups. For example, in response to questions regarding their participation in The Council, several youth responded with "N/A", possibly indicating that they had not attended a single group. Other youth were more specific, stating for example, "nothing cause we aint never had a group wit the council" and "never attended". These responses, if not taken out in the first phase of the qualitative analysis (e.g., 'N/A'), fall under the theme 'Boys Council.' This finding that not all youth at the experimental facilities participated in The Council helps explain why there is no effect of participation when lumping all youth from the experimental facilities together, but there is an effect when we parcel out the attendance in the program. In other words, the youth that have not received any Council curricula have contaminated the dichotomous effect of the program, explaining, in part, why there was no program effect, whereas youth with zero hours of attendance were screened out of the dosage effect model, which assessed the effect of the amount of attendance on changes in level of adherence to traditional masculinity ideology over time.

In addition to aiding in the interpretation of the somewhat discrepant program effectiveness findings, the qualitative analysis also helped evaluate the trustworthiness of the effect of attendance. In particular, youth responded to questions about what they learned in The Council or about being male in ways that may illustrate a decrease in level of adherence to traditional masculinity ideology. 
For example, the theme 'Emotional Awareness' described responses such as, "I changed as an individual by learning to express myself to other and not hold anything back" and "I learned you ain't gotta hide yo feelings just because your male it's ok to express feelings", which are indicative that youth are changing in ways that oppose traditional masculine ideals. In addition to this theme, the themes 'Physical Awareness,' 'Man Up,' 'New Perspective,' and 'Relational' each describe responses that are also illustrative of ways in which youth oppose aspects of traditional masculinity ideology.

Hypothesis 2. Age Effect

H2a. Age will predict adherence to masculinity ideology at baseline.

H2b. Age will be a negative predictor of linear change in adherence to traditional masculinity ideology.

Results from the quantitative analyses support the first hypothesis that age was related to adherence to traditional masculinity ideology at baseline. However, the direction of the relationship, negative, was opposite to the positive relationship that was hypothesized. Younger youth in the sample adhered more strongly to traditional masculinity ideology, whereas older youth adhere less strongly. Despite this finding, age was not predictive of change in level of adherence to traditional masculinity ideology over time.

Though the qualitative findings were not linked to the age of the participant, the first phase of the qualitative analysis allowed me to read the same youths response after 10-weeks in The Council and then again after 20-weeks. Thus, I was 
provided a better understanding of how youth describe what they have learned about being male from their participation in the program over time. Clearly, these responses are confounded by a youth's participation in The Council, but it also speaks to the dynamic nature of youth's responses over time. This, in turn, may help with the interpretation of the null effect of age on level of adherence over time. For example, in response to the question, "What have you learned about being male?" after 10-weeks one youth stated, "be responsible for my actions" and "acting my age being responsible" after 20-weeks. Another youth responded, "MOST OF US ARE IN THE SAME SITUATIONS” after 10-weeks and "WE THINK ALMOST THE SAME" after 20-weeks. These youth are focused on the same aspect of being male over the course of the study. Thus, these responses support and illustrate stability in how youth describe what they have learned about being male. The stability in change of adherence to traditional masculinity ideology as predicted by age may be illustrated by these similar responses that are made by the same youth over the course of the study.

Despite the stability illustrated with the responses above, other youth provide varying responses over time. Additionally, the quantitative assessment of the first hypothesis stated above found that age is related to initial level of adherence. Therefore, it may be deduced that level of adherence to traditional masculinity ideology is in fact dynamic over the course of adolescence. Though, change may not be apparent over only 20-weeks, as is illustrated with some of the static responses. On the other hand, as is suggested by the open-ended responses, the patterning of 
change over time may be different for each individual youth, such that the nuanced changes that occur over a short time period for some youth may be contaminated by some youth who are going through a relatively stable period.

Hypothesis 3: Race/Ethnicity Effect

H3a. Race/Ethnic identity will predict adherence to traditional masculinity ideology at baseline.

Hypothesis 3, part a, posited that race/ethnic identity predicts initial level of adherence to traditional masculinity ideology over time.

H3b. Race/Ethnic identity predicts masculinity ideology development (neutral hypothesis).

Results of the quantitative analysis found White youth to have lower levels of adherence to traditional masculinity ideology at baseline, but increase at a greater rate compared to African American youth. However, Latino youth were not statistically different from African American youth at baseline or over time. Despite the fact that the qualitative findings were not linked to the race/ethnic identity of the youth, several responses, in particular those coded under the theme 'Man Up' are reminiscent of the cool pose script described by Majors and Billson (1992) or machismo (e.g., Kimmel, 2007), which I presented in greater detail in Chapter III.

Like cool pose and machismo, 'Man Up', a phrase that several youth used in their responses to questions regarding their participation in The Council and what they learned about being male, refers to a restricted form of masculinity. In an attempt to better understand the meaning behind the phrase 'Man up', I looked it up 
in the Urban Dictionary ${ }^{3}$ (November 19, 2011) and provide the definitions here. Man up refers to several different expectations, including “(1) Don’t be a pussy, brave it, be daring; (2) To fulfill your responsibilities as a man, despite your insecurities and constant ability to place yourself in embarrassing and un-manly scenarios; (3) Be strong; (4) strap on a pair, grow some balls, stop being such a complete and utter wuss; (5) To work through impediments and obstacles without whining; (6) Derived from the phrase 'cowboy up', meaning 'be tough, be strong, act like a real cowboy', which has been in use in rodeo circles since at least the mid-1970's; (7) Man up can also mean that an individual to be (not 'act') mature, to grow up, quit being childish, change their ways and turn around (do a "180") and to go the correct way from now on, (8) That someone stop being self-centered, look at what they are doing, how they are acting, and change viewpoints, and perspectives on the situation(s) and move forward correctly in their life, and/or others lives; (9) That one be a leader, to step up to the plate when no one else will, to give it your best shot, to TRY!".

As is evident in most of the definitions above, 'Man up' refers to a script that men are expected to perform, much like that of cool pose and machismo. Additionally, as Kimmel (2007) described, men in situations or cultures in which they feel powerless often present themselves in ways that embody machismo and/or cool pose. Likewise, 'Man up' may be performed in a prison setting where the inmates feel powerless. Despite the similarities between alternative masculine performances, such as cool pose and machismo, I cannot determine whether 'Man up' is an expectation that youth of a single race or ethnicity describe. 


\section{Hypothesis 4: Ethnic Pride Effect}

H4a. Ethnic pride will positively predict adherence to masculinity ideology at baseline.

H4b. The relationship between ethnic identity and adherence to masculinity ideology over time will be moderated by ethnic pride.

Results indicate that African American youth with high levels of ethnic pride report lower levels of adherence to traditional masculinity ideology at baseline, compared to those with low levels of ethnic pride. This effect was not detected for White or Latino youth. Additionally, ethnic pride was unrelated to change in level of adherence to traditional masculinity ideology over time.

The obvious explanation of these findings is that African American youth with high ethnic pride adhere less to traditional masculinity ideology than those with low ethnic pride. However, one possible alternative explanation may also be made. For example, as was described above, the theme 'Man up' seems to be a phrase that explains an alternative, but restricted form of masculinity, like cool pose or machismo that some youth in the juvenile detention facilities are expected to perform. Like the many different versions of the definition to the phrase 'Man up', youth in this sample may have several different alternative forms of masculinity ideology that are not fully assessed by the quantitative measure. For example, African American adolescents with high levels of ethnic pride may adhere to an alternative form of masculinity ideology that it somewhat opposed to that of traditional masculinity, as measured by AMIRS (Chu et al., 2005) and as such their 
level of adherence is less than those with lower levels of ethnic pride. Moreover, White and Latino youth with high ethnic pride may adhere to an alternative form that does not parallel traditional masculinity ideology, as measured by AMIRS.

Therefore, regardless of the level of ethnic pride, White and Latino youth are not different in their level of adherence to traditional masculinity, but may vary in their adherence to an alternative masculinity.

Unfortunately, beyond the idea that 'Man up' brings to the table — that there are multiple different forms of masculinity ideology — the qualitative themes do not provide much additional insight to these hypotheses. However, a handful of youth make reference to their race/ethnic backgrounds in responses to the open-ended questions. For example, in response to the question about what youth learned about being male, a response such as 'I have learned being a male we have a lot of virtues in like especially being a black male we have it harder then some people but we also have a lot of virtues to' or 'I hate J.C.O. 's At [location name].They Are Racist.' may provide some insight to the different realities that are experienced by young men of different race/ethnic backgrounds and varying levels of ethnic pride. In particular, the first response suggests that Black youth do not separate their gender from their race/ethnicity in ways that other youth, in particular White youth, do. This is consistent with theory regarding race/ethnic salience for people of color (e.g., Phinney, 1996). So, when being asked about gender, Black youth tend to think about the intersection of race/ethnicity with their gender, whereas White youth may not. The second response suggests that youth of color may have unique experiences 
within the prison facilities based on the way they are differentially treated by juvenile correction officials (J.C.O's). Thus, highlighting the importance of investigation into this research question.

Hypothesis 5: Prison Effect

H5a. Days in Prison will positively predict initial levels of masculinity ideology.

H5b. Days in Prison will negatively predict change in masculinity ideology.

The number of days youth had been at the juvenile justice system had no effect on their initial levels of adherence to traditional masculinity or their trajectories of change over time. One possible explanation for not detecting this effect may be due to the differing experiences and teachings about masculinity and expected male behavior that youth have absorbed during their time in the prison. As defined above, youth in the experimental facilities describe multiple different malerelated themes. These themes were generated by responses that youth provided to their experiences in The Council. Interestingly, however, some of the themes oppose one another, illustrating that youth participating in the same program, take away different messages. For example, under the theme 'Emotional Awareness' are two paradoxical subthemes, 'Emotional expression' and 'Emotional inexpression'. Like the experiences of some youth in The Council, youth in prison may be experiencing different realities and expectations of being male. Thus, the number of days in prison, alone, may not account for their varied experiences. 
Summary. To summarize, youth have qualitatively described what they have gained (or lost) from their participation in The Council, in particular, what they have learned about being male. I carefully coded the data into 8 distinct, but related themes. In the section above, I have described how these themes and how individual responses may provide insight to the quantitative findings. I have briefly described how these themes may inform each of the studies hypotheses. In the chapter to follow, these findings are reviewed and brought into conversation with the literature presented in the first three chapters. I conclude with an explanation of how these findings inform current theories of masculinity ideology development and the practice of gender-specific program implementation in juvenile justice facilities. 


\section{CHAPTER VIII}

\section{Discussion}

The purpose of this dissertation was to describe the developmental trajectories of adherence to traditional masculinity ideology for adolescent inmates. The dissertation, in part, examined the effect of a strength-based program, The Council for Boys and Young Men, in successfully affecting change in level of adherence to traditional masculinity ideology. The dissertation assessed predictors of initial levels of masculinity ideology, at the beginning of the study before youth at the experimental facilities began participating in The Council, as well as the antecedents of change in level of adherence to traditional masculinity ideology over time. In addition, the research explored how adolescents' qualitative ideas about being male inform their quantitative developmental trajectories of masculinity ideology. In the following sections, I review the study's findings and connect these findings to the literature reviewed in the prior chapters. First, I describe the effect of participation in The Council (Hypothesis 1). Next, I discuss the effect of participants' age (Hypothesis 2), race/ethnic identity, ethnic pride (Hypotheses $3 \& 4$ ), and length of time in prison (Hypothesis 5) on changes in masculinity ideology. As I discuss the results of each hypothesis, I describe the findings of the Mixed-Method Analysis, where the qualitative findings were used to help evaluate and aid in the interpretation of the quantitative results. Embedded in this review as I connect the findings to the literature reviewed in previous chapters, I briefly describe some of the implications of each finding. Following a description of the results of the hypothesis testing, I 
review the qualitative themes identified through analysis of youth's responses regarding their participation in The Council and what they learned about being male (Research Question 2). To end, I discuss the study's overall limitations and strengths, and conclude with a consolidated review of the study's implications accompanied by suggestions for future research directions in the field.

\section{Research Question 1}

Program Effect. The effect of participation in The Council was assessed in the final statistical model (Model 7, Table 11g) presented in Chapter VII, comparing youth in prison locations that were implementing the program with youth from the control locations. Additionally, a dosage effect was assessed in a separate model to determine whether attendance in The Council had an effect on changes in masculinity ideology over time for youth in the experimental locations. Although youth in the experimental facilities had initially higher levels of masculinity ideology than those in the control locations, change over time was not different across the groups. In other words, whether or not youth participated in the program had no effect on their level of adherence to traditional masculinity ideology over time. However, for youth in the experimental locations who were exposed to the program, the amount of participation (attendance) in The Council did have an effect on change. Specifically, although youth, in general, tended to show an increase in level of masculinity ideology over time, those who attended a greater number of The Council sessions increased at a lower rate over the first 10-weeks and second 10weeks of the program, as compared to youth who attended fewer sessions. 
These seemingly discrepant findings on the effectiveness of The Council at affecting change in level of adherence of traditional masculinity, can be more easily interpreted when brought into conversation with the qualitative responses, as was accomplished through the Mixed-Method Analysis. Specifically, qualitative responses made it known that not all youth at the experimental facilities attended The Council. In fact, some youth qualitatively describe not having attended any groups. Thus, the dichotomous program effect comparing trajectories of masculinity ideology development across study conditions was contaminated by youth who were placed in the experimental group on the basis that they lived within the one of two experimental facilities, but had not received the "experiment", The Council. In other words, the qualitative analyses highlighted an artifact of the quantitative 'Intent to Treat Design', that not all youth directly participated in the program. Fortunately, a dosage effect or the effect of attendance in The Council on level of adherence of traditional masculinity ideology was also assessed. Before examining the dosage effect model, all youth with zero hours of attendance or no attendance in the program were removed from analyses. Thus, this analysis provided a more sensitive assessment of the effect of the program and was ultimately able to detect the predicted relationship.

The finding that change in masculinity ideology is effected by program dosage has important implications for the continued implementation of The Council at ODYS facilities and other prison locations. Because adherence to traditional masculinity ideology has been linked both to poor health and behavior outcomes 
(Levant \& Richmond, 2007; O’Neil, 2008), a program's effectiveness at decreasing levels of adherence (or weakening the level of increase) is practically important. In particular, youth in the current study are all convicted felons incarcerated in the juvenile justice system. Thus, it is likely that these youth demonstrated behaviors (e.g., violent or aggressive behaviors) that are consistent with traditional masculine norms that resulted in their incarceration. As was described in Chapter III, the United States Juvenile Justice System has recently taken a strengths-based approach to focus on positive youth development instead of the traditional deficits approach (Barton \& Butts, 2008). Therefore, the study's finding that participation in a strength-based program had an effect on changes in level of adherence to traditional masculinity that is associated with problem behaviors provides some evidence that this type of programming may be effective in this context. The definition of "effectiveness" in this study must be interpreted with caution, however, as it is not to say that youth are becoming less violent, being released from the system, or not recidivating. It does, on the other hand, speak to some ideological aspects of youth development that may be associated with these aspects of juvenile justice system success and thus, warrants further investigation.

Furthermore, this finding may be surprising given the descriptions of correctional environments as "not naturally amenable to a strength-based paradigm" (Barton \& Butts, 2008, p. 13) and a place where strength-based perspectives compete with a rooted traditional deficit-based environment (Abrams et al., 2005). In fact, these competing factors were apparent in youth's qualitative descriptions of what 
they learned from the program. For example, a thematic analysis of the qualitative data picked up on a paradoxical environment in which some youth describe a freedom of expressing emotion and encouragement of emotional expression, while at the same time other youth describe the need to be emotionally stoic. One potential explanation for this finding given the literature described above is that although youth are receiving strength-based programming that encourages emotional expression through challenging traditional masculinity stereotypes, this program, The Council, comprises only a small portion of their overall time in the system. To be clear, even for youth attending $100 \%$ of The Council groups, this program only accounts for 2 of the $268(1 \%)$ hours in a single week. The remaining 266 hours youth spend in their cell, the shower area, exercise room, other programming and school settings. In these other environments and settings, these strength-based messages that focus on encouraging healthy masculine identity development may be absent. Moreover, though The Council curricula message regarding masculinity is clear, the facilitators of the program may have personal ideals that counter these messages. Therefore, although there is some evidence that strength-based, gender specific programming may be effective in juvenile justice facilities, the continued investigation into strength-based programming, and the moderating effects of environmental norms and fidelity of program implementation is needed.

Age Effect. Literature assessing the nature of the relationship between age and adherence to traditional masculinity ideology is mixed, with some studies reporting a negative relationship (Levant et al., 1992; Pleck et al., 1994) and others 
reporting a positive relationship (Abreu et al., 2000). In the current study, the relationship between age and level of adherence to traditional masculinity was hypothesized to be positive because of the sample's demographic similarity to Abreu and colleagues' sample. However, the effect detected was in the opposite direction than hypothesized and is more consistent with Levant and colleagues' findings in a sample of undergraduate students that age and adherence to traditional male role norms are negatively related. Specifically, the current study found that older participants reported lower levels of adherence to traditional masculinity at baseline compared to younger participants. In other words, age was found to be negatively related to level of adherence to traditional masculinity ideology.

Despite detecting this relationship between age and level of adherence to traditional masculinity ideology, age was not shown to predict change in masculinity ideology over time. Thus, although a relationship between age and level of masculinity ideology was found, change in level of adherence to traditional masculinity ideology over the course of 20 weeks was not determined.

The effect of age on initial levels of traditional masculinity ideology was moderated by prison facilities. Specifically, youth from the experimental locations had a weaker negative relationship between age and initial levels of adherence to traditional masculinity ideology, whereas youth from the control sites showed a stronger negative relationship. Though geographic location has been shown to be a moderating factor in other characteristic influences on traditional masculinity (e.g., race/ethnicity), this has not been clearly demonstrated in a single study with age. 
Although the prison locations are all in the state of Ohio, the facilities differ in some specific and concrete ways, as was described in Chapter VI, Methods. Additionally, in the current study facilities differ on the average age of inmates. Specifically, the average age at the experimental locations $(M=17.11)$ is older than that of the control locations $(M=16.64)$. These differences in average age in combination with the moderating effect, may suggest a curvilinear relationship between age and adherence to traditional masculinity ideology. For example, in earlier adolescence, level of adherence to traditional masculinity ideology may decrease at a greater rate than later in adolescence. Perhaps the differing rates of change in adherence to traditional masculinity ideology over the course of one's lifetime may also help explain why the literature on age and masculinity ideology is mixed. Still, the data did not support this theory and further investigation into the trajectories of change in level of adherence to traditional masculinity ideology is warranted. In particular, assessing change over a greater length of time may allow for the detection of this effect.

Even with the restricted range of age in the current sample, age was an important predictor of initial levels of adherence to traditional masculinity ideology. This finding lends some understanding to the developmental nature of masculinity ideology. In particular, older adolescents in the study tend to adhere less strongly to traditional masculine ideology than younger adolescents. One set of possible explanations for this relationship may be informed by the developmental psychological framework of adolescence - biological, psychological, and 
sociological lens (Cobb, 1992). Though these lenses overlap, I have explained them separately below so that their unique implications may be made clear.

Through a biological lens, puberty status and puberty timing may be important explanations as to why younger adolescents report having higher levels of adherence to traditional masculinity ideology compared to older adolescents. In particular, younger youth who have not yet reached puberty are physically smaller, their voices higher, and their genitals and faces are hairless, characteristics that may be considered "boy-like" or "feminine". However, older youth who have reached puberty are physically bigger and stronger, have deeper voices, and may have hair both covering their genitals and faces. Given the context of this study, youth may be particularly aware of each other's puberty status through constant contact including showering together, exercising together, and sleeping in the same quarters. Thus, youth who have reached puberty feel less of a need to adhere strongly to traditional masculine ideals, given their physical body is more representative of a mans compared to the younger youth who have more boyish or feminine features.

Through a psychological lens, adolescence is a period in life in which an individual achieves a continuing and stable sense of self (Havinghurst, 1972). Thus, the negative relationship between age and traditional masculinity ideology may be explained in relation to identity and sense of self development. Specifically, younger youth may have less stable sense of self and therefore may adhere more strongly to external messages regarding gender identity, whereas older youth with a stronger sense of self can turn inward to determine a gender identity. 
Finally, through a sociological lens, adolescence is defined as a period in life in which an individual transitions a role that is dependent to one that is independent (Cobb, 1992). However, in the context of this study, youth are never able to achieve independence as they are dependent on the system for food and shelter and they are not awarded the same legislative privileges as other adolescents, such as driving or voting. With that said, the prison system may use age cohort to separate youth into different groups and classrooms. Thus, older youth may be awarded certain aspects of independence that are not awarded younger youth. If this were the case, older adolescents may feel less need to adhere to normative traditional masculinity because their day-to-day life allows them to enact aspects of traditional masculinity that are more salient. These potential "awards" are unclear in the current study, but are worth exploring in future research.

Another possible explanation of the relationship between age and masculinity ideology development may be informed by the trajectory of change over time as predicted by age. Specifically, the lack of change in masculinity identity over 20weeks and the stable within participant responses that were described in the MixedMethod Analysis sheds light on its possibly dynamic nature. These findings suggest three possibilities about change in masculinity ideology over time: (1) change in masculinity ideology is slow and cannot be detected over the course of 20 -weeks; and/or (2) change in masculinity ideology is multidimensional and difficult to determine with only three measurement points; and/or (3) change in masculinity ideology is differing for different youth, such that some youth throughout 
adolescence do not show change, whereas others increase or decrease in level of adherence at differing rates, and when combined together these effects counteract one another. In any case, in this study, after controlling for other study variables, change was detected for some youth. Therefore, the trajectory of change in level of adherence to traditional masculinity ideology over the period of adolescence is likely complex and is likely influenced by both personal (e.g., physical and psychological) and environmental (e.g., sociological, contextual) factors, some of which were explored and detected in this study.

Race/Ethnic Identity and Ethnic Pride. Similar to the study of age and masculinity, the literature is mixed when it comes to describing racial/ethnic group differences in level of traditional masculinity. For example, whereas Levant and Richmond (2007) report higher levels of adherence to traditional masculinity ideology among African Americans, followed by Latinos and lastly, Whites, whereas Abreu and colleagues' (2005) reported higher levels of adherence among Latinos, followed by White and lastly, African Americans. In the current study, White participants had lower initial levels of adherence to traditional masculinity ideology compared to the African American youth. This finding is consistent with most of the literature on ethnicity and masculinity ideology (e.g., Levant \& Majors; Levant \& Richmond), though not with others (e.g., Abreu et al.) In addition to detecting this effect, the current study found group differences in trajectories of change in level of adherence to traditional masculinity ideology over time. Specifically, although White youth had lower initial levels of adherence, they increased at a greater rate compared 
to African American youth. And, though differences were detected between White and African American youth, no differences were determined between Latino youth and African American youth at baseline or change over time.

In addition, African American participants with greater levels of ethnic pride reported lower initial levels of adherence to traditional masculinity ideology. Interestingly, this relationship is in the opposite direction than the relationship between ethnic belonging and traditional masculinity reported by Abreu and colleagues (2005), and was not detected for Latino or White youth in this study. In other words, whereas Abreu and colleagues posit that the development of ethnic belonging and masculinity is the same for all youth across racial/ethnic categories, the current study did not find this to be the case, but instead found this relationship to differ by racial/ethnic category.

One possible explanation for the finding that African American youth had higher levels of traditional masculinity ideology compared to White youth, but that level of adherence to traditional masculinity ideology was moderated by level of ethnic pride, may be informed by literature on ethnic identity salience. In particular, Phinney (1996) described ethnic identity as "an enduring, fundamental aspect of the self... to the extent that it has salience and centrality of the individuals involved." (p. 922). The amount of importance and strength of ethnic identity varies both within and between ethnic groups. In particular, most Americans of European background are described as not experiencing ethnicity as a salient and important aspect of their identity, whereas ethnicity tends to be more important to individuals of color 
(Phinney). Research suggests that greater importance ethnic identity plays, the larger the contribution it makes to one's self-concept. Moreover, ethnic identity is considered a developmental process in which achieving an ethnic identity is said to occur in individuals that are secure and confident in their self and as a member of their ethnic group (Phinney). Given the literature described, race/ethnic identity for White youth in the study may not be an important aspect of their overall identity. Thus, aspect of gender identity may take greater importance and as identity develops over time, gender identity may become more and more important to this group. For this reason, White youth may be looking to external references for guidance in developing their gender identity. The study's finding that White youth's level of adherence to traditional masculinity ideology increased over time relative to African American youth, is consistent with Phinney's theory. On the other hand, race/ethnic identity may be of greater importance for African American youth's identity and as such, those with greater levels of ethnic pride place less importance on gender identity development (or belonging to a group, such as one with high traditional masculine ideals).

Furthermore, the finding that African American adolescents with higher levels of ethnic pride adhered less strongly to traditional masculinity ideology than did African American youth with lower levels of ethnic pride suggests the need for researchers to consider the importance and meaning of sub-cultural variations in masculinity ideology development within cultural groups that are defined by race/ethnicity. Because African American men were not given access to the same 
traditional masculine ideals that were afforded to White men, African American men created alternative performances of masculinity (e.g., cool pose) (e.g., Kimmel, 2007; Majors \& Billson, 1992). Similarly, African American adolescents in the current sample with higher levels of ethnic pride may adhere to an alternative form of masculinity that is not assessed by the quantitative measure of adherence to traditional masculinity ideology. On the other hand, African American youth in this sample who had lower levels of ethnic pride adhere more strongly to traditional masculinity ideology, and may adhere less so to the alternative forms of masculinity. For example, youth in the study describe an alternative form of masculinity, 'Man up' that seemingly refers to a complex and varied form of masculinity. Though the qualitative responses were not connected to an individuals' race/ethnicity or level of ethnic pride, both the traditional and alternative descriptions of what the youth have learned about being male, suggest the need for further investigation into alternative forms of masculinity ideology among boys and men of different backgrounds.

Prison Effect. Despite the descriptions of "the culture of" traditional masculinity occurring in juvenile justice facilities (Abrams et al., 2008; Cesaroni \& Alvi, 2010), the number of days youth in this sample had been in prison was not related to initial levels of adherence to traditional masculinity ideology. Nor was it found to influence the trajectory of change over time as predicted. There are several possible explanations as to why this effect was not detected. For example, the assessment of traditional masculinity ideology was limited to one 12-item self-report survey that may not capture the full experiences of the youth, as was suggested in the 
Mixed-Method Analysis. Another plausible explanation is that the number of days in prison is not be linearly related to adherence to traditional masculinity ideology. Finally, the relationship between the number of days in prison and adherence to traditional masculinity ideology may be confounded by a third variable that was not included in the study. The lack of effect detected in the current study, in conjunction with support from the literature regarding this relationship, suggests the need for further investigation into the culture of masculinity in juvenile justice facilities using a combination of quantitative and qualitative observations over time.

\section{Research Question 2}

Open-ended responses provide a picture of what youth learn about being male as a complex and multifaceted role, expectation, and experience. The eight themes identified in the qualitative analyses were: (1) Emotional Awareness: "male's have feelings to[o]" vs "cant let nobody see you cry"; (2) Gender Awareness: "man do what they want" vs. "Respect women"; (3) Physical Awareness: "you dont have to be a punk if you walk away from a fight" vs. "we are sopost to be strong"; (4) Leadership, Status, \& Self-Efficacy: "be a role model!”, "doung negative stuff does not make you any cooler than anybody else", \& "I fEE likE I can go far in lifE"; (5) "Boys Council”; (6) "Man Up"; (7) New Perspective: "keep my head up" \& "my action can hurt other people"; and (8) Relational: "Healthy Relationships" \& "A strong man... can do A lot to help people". Briefly, the theme Emotional Awareness encompasses responses that describe both a lack of emotional response and emotional expression. Responses that fall under this theme support the finding from 
hypothesis $1 \mathrm{~b}$ that several youth attending The Council are learning about how to be male in an alternative and flexible way that opposes the traditional masculinity ideology script. Gender Awareness refers to responses regarding male dominance and/or descriptions of gender differences or gender role expectations. Responses that fall under this theme bring to light the varying experiences of youth in The Council. In particular, some youth continue to express opinions that are inconsistent with The Council messages, and consistent with traditional masculinity ideology. Physical Awareness is used to describe responses regarding an avoidance of conflict and fights, as well as the expectation to be tough and strong. These responses encompass youth who express learning that men are not expected to be tough and violent, which goes counter to the message of traditional masculinity ideology. However, at the same time, some youth are not learning alternative, nonviolent forms of masculinity. The theme competitive and ambition encompasses three subthemes including leadership, status, and self-efficacy. Responses under this theme are similar to some aspects that are covered under traditional masculinity ideology. These responses may help explain why level of adherence to traditional masculinity ideology increases over the course of the study for some youth. "Boys Council" is a theme that captured responses regarding participation in the program that did not directly pertain to the research question. However, this theme represented some of the responses that were used to illustrate the quantitative findings that evaluated the effect of The Council, and thus, are important in the understanding of hypothesis 1a-b. The theme "Man $U p$ ” is a general theme to capture all masculinity-related responses that were not 
specific enough to be coded under another theme. Though this theme is broad, it provides a unique insight into responses that do not easily enhance or illustrate any specific quantitative hypothesis. Despite this, this theme plays an important role in illustrating some potential gaps in the quantitative assessment of masculinity ideology. In particular, this theme was used to describe a possible alternative form of masculinity ideology in hypotheses 3-4. Finally, New Perspective encompasses responses regarding personal growth, awareness, and new perspectives gained through participation in The Council. Like Emotional Awareness, the responses under this theme support the finding that The Council was effective at decreasing level of adherence to traditional masculinity ideology for youth who attended.

Interestingly, several of the constructs assessed by the Adolescent Masculine Identity in Relationships Scale (AMIRS) (Chu et al., 2005) measure of masculinity ideology that was used in this study were also discussed by the youth in when asked about their experiences in The Council. In particular, emotional stoicism, heterosexual dominance, physical toughness, competitiveness, and ambition are all concepts that are measured by AMIRS and were identified as themes or subthemes in the qualitative analysis of the youths' responses. Thus, the measure captures much of what the youth describe as contemporary and important concepts regarding masculinity and masculine expectations. However, youths' descriptions of what they have learned about being male, though thin, were more complex and dynamic than can be assessed in a 12-item quantitative measure. 
As an example provided below highlights, there are some gaps in the quantitative measurement that are filled out in part by the qualitative responses by youth. For example, two items on the AMIRS asks youth about their feelings on avoiding fights - "I can respect a guy who backs down from a fight" and "A guy never needs to hit another guy to get respect". Though these items make up nearly $20 \%$ of the entire scale, they both get at very similar concepts regarding fighting and respect. The youth, on the other hand, described multiple different concepts relating to fighting in addition to that of respect. Most often, youth described gaining the skills needed to avoid fights and controlling anger through their participation in The Council. These skills are immediately necessary to avoid fights and are behavioral in nature, but do not get at the attitudinal perspective of fighting in the same way as the AMIRS items listed above. Still, some youth described a connection between avoiding fights and being respected, but not as often as they describe how they themselves have learned to avoid fight. In this example, some youth describe changing in ways that would parallel a decrease in traditional masculinity ideology due to their participation in The Council. However, after controlling for other quantitative study variables (e.g., age, ethnic identity, ethnic pride, location), the level of traditional masculinity ideology over the course of the study was the same for youth in the experimental group compared to the control. Perhaps if masculinity ideology were assessed with multiple different measures for each concept of traditional masculinity ideology, this measure might be more sensitive to changes youth describe experiencing (e.g., skills they have learned to avoid fights). 
In addition to potential gaps in measurement that were identified through the qualitative analysis, youth describe an alternative form of masculinity ideology that was depicted in the theme "Man Up". Though several aspects of the definition and description of "Man Up" parallel the constructs of traditional masculinity ideology described in Chapter III, there are nuances to "Man Up" that are worth exploring. For example, "Man Up" appears to provide a level of flexibility that is counter to that of traditional masculinity. In particular, "Man Up" includes the definition "That someone stop being self-centered, look at what they are doing, how they are acting, and change viewpoints, and perspectives on the situation(s) and move forward correctly in their life and/or others lives" (Urban Dictionary, 2011). This aspect of the definition of "Man Up" was also described by youth and categorized under the theme "Relational". As was described in Chapter III, the inflexibility and the experience of gender role conflict as a result of adhering strongly to traditional masculine ideals is what is believed to contribute to poor health and behavioral outcomes. Therefore, alternative perspectives of masculine ideals, such as the one identified in this study, "Man Up", may provide youth with greater flexibility and less rigidity compared to what is experienced when adherence to traditional masculinity ideology is high. As previously described, Pollack (1996) found boys in his study to support both egalitarian and traditional male norms, which is similarly consistent with the notion that youth may adhere to alternative aspects of masculinity ideology as the current study suggests. In fact, the qualitative analysis supports the idea that contemporary notions of masculine ideals may be moving beyond that of 
traditional masculine ideals. For these reasons, future studies should examine how masculine ideals have evolved for youth over time and how contemporary ideals differ from traditional ideals in their influence on health and behavioral outcomes.

\section{Conclusion}

Taken together the findings from this dissertation lend support to theories of multiple masculinities (e.g., Smiler, 2004). That is, the study's findings suggest that adherence to traditional masculinity ideology and descriptions of masculinity are developed, maintained, and restructured according to one's personal, social and environmental contexts. Specifically, in this study, younger adolescents who identified as African American, and who were located in the experimental study locations had higher levels of adherence than older adolescents, who identified as White, and who were located at the control study locations. However, within these groups, additional social and environmental factors influenced level of adherence and descriptions of masculinity. For example, although White youth initially adhered less strongly to traditional masculine ideals, their level of adherence increased at a greater rate than African American youth. Moreover, African American youth with high levels of ethnic pride had lower levels of adherence to those ideals than those with low levels of ethnic pride. Finally, youth at the experimental prison locations adhered more strongly to traditional masculine ideals, as compared to those at the control locations. However, youth with greater participation in The Council experienced less dramatic increases in level of adherence to traditional masculinity ideology over time compared to those with less participation. Finally, in addition to 
youth's descriptions of masculinity and changes in masculinity that paralleled concepts that were measured quantitatively, youth described aspects of masculinity ideology that were not quantitatively assessed. These descriptions were diverse, and one youth's response sometimes opposed that of another. Qualitative responses were coded into themes that provided illustrations and support to the study's quantitative findings and insight as to why a hypothesized effect was not supported by the data. For example, the significant dosage effect finding (hypothesis $1 \mathrm{~b}$ ) was enhanced by youth descriptions of what they learned through their participation in The Council. Additionally, through youth's responses, it was understood that not all youth in the experimental facilities participated in The Council. Thus, the program effect (hypothesis 1a), as modeled using an "Intent to Treat" design, was contaminated by the non-participating youth in the experimental facilitates. To conclude, this study lends support to the idea that personal, social, and environmental factors influence the development of masculinity ideology in adolescent males.

\section{Potential Limitations}

The study provides results of an evaluation of a strength-based program that does not consider the fidelity of the program implementation and therefore these results must be interpreted with caution. While the study offers some hopeful preliminary findings, they need to be further examined in conjunction with a program implementation evaluation study. Specifically, results from the first hypothesis (program and dosage effect) are affected by this limitation and it remains unclear what aspects of the program influenced youth's trajectory of change in level 
of adherence to traditional masculinity ideology. In addition to this limitation, there are several other important limitations of the study that are described in detail below.

A second important limitation concerns the operationalization of the construct of race/ethnicity. Though racial/ethnic categories are sometimes useful for labeling purposes, measurement, analysis, and discussion, they are inherently flawed. Race/ethnicity, as noted in the review of literature in Chapter IV, is inherently a multidimensional construct (e.g., Phinney, 1996). However, in the current study, identification with a specific category was assessed with one item, which clearly cannot capture all aspects of race/ethnicity. Additionally, the coarse categorization inevitably encompasses a heterogeneous group of adolescents, from different neighborhoods, communities, and cultural backgrounds. Thus, the meaning derived from the influence of racial/ethnic identity on masculinity ideology development is limited to only that of a broad descriptiveness between group differences. Only the variation in the dimension of ethnic pride is assessed within group. All other withingroup variation is unexplored.

Despite the limitations to the categorization of race/ethnicity noted above, differences between African American and White youth were detected, as were variations among African American youth as a function of level of ethnic belonging. Although these results must be interpreted with caution, given the heterogeneity of each racial/ethnic group, it is important to point out that youth self-identified their race/ethnicity in the study. Because the race/ethnic categories African American, Latino, and White were self-identified and mutually exclusive (all mixed-race/ethnic 
self-identified participants were placed into the 'Other' category), the differences identified have face validity and provide an important starting place into an important investigation of masculinity ideology developmental differences among youth of different race/ethnic backgrounds.

A third limitation of the current study is the reliance on a non-random sample and thus the introduction of sampling error. Using Groves (2006) structure to identify sources of sampling error, the target population of this study is youth incarcerated in juvenile justice facilities in the United States. The frame population included youth incarcerated in ODYS facilities (identified using their ODYS numbers). The sample included youth within four of the five facilities invited to participate in the study. Finally, respondents include only a percentage of the sample that completed at least one survey measurement. It is unclear what percentage of youth voluntarily declined to participate in the study and what percentage of youth was unable to participate in the study due to external constraints such as being held in solitary confinement. Thus, coverage error, a specific type of sampling error, was introduced via the imperfect sampling of the target population. Coverage error may introduce bias into the findings if the youth who participated in the study differ from those who did not participate. In particular, coverage error may have influenced the assessment of the effect of time in prison. If youth who did not participate are provided limited access to programming as the result of problem behavior that lead to solitary confinement, outcomes that assess the impact of prison on level of adherence to traditional masculinity ideology may be limited. Therefore, the 
generalizability of the study is limited. Though limited, the findings from this study come from a relatively diverse and large sample of adolescents in prison in the state of Ohio. Therefore, the study's findings are relevant to ODYS inmates and may be used to inform future studies in juvenile justice facilities across the United States.

A fourth limitation of the current study was introduced due to the nonrandomized experimental design. Given the study does not adhere to a true experimental design, conclusions regarding the efficacy of the program, The Council, on decreasing adherence to traditional masculinity ideology cannot be made with certainty. For example, youth in ODYS participate in additional programming, including one or more of the following: Strength-Based Behavioral Management System; Cognitive Behavioral Therapy; Thinking for Change; Anger Management; Victim Awareness; Relapse Prevention; Substance Abuse Education; Chemical Dependency Intervention; and Sex Offender Programming. However, if the additional programming influenced masculinity ideology, it should influence the outcome similarly across locations because the additional programming is the same. However, as was described in the Methods Chapter, each facility is unique in some concrete ways. These differences were noted when youth at the experimental facilities were found to have higher initial levels of adherence to traditional masculinity ideology compared to those at the control facilities. Additionally, the differences in facilities were illuminated with a moderating effect of age and level of adherence to traditional masculinity. The negative relationship between age and level 
of adherence to traditional masculinity ideology was weaker at the experimental facilities and stronger at the control facilities.

In addition to the lack of randomization, the study did not assess the implementation of The Council within the experimental facilities. Therefore, from the data alone, there is no way to determine whether youth attended groups that were facilitated by social workers who rigorously followed the curriculum and supported the messages of the program or attended groups facilitated by social workers who took liberty to adapt the curriculum and did not support the messages of the program. With out a measure of program implementation fidelity the conclusions that can be made from the findings for hypotheses $1 \mathrm{a}$ and $1 \mathrm{~b}$ are limited. Future studies may consider measuring fidelity of program implementation from the facilitator and the youth's perspectives, in addition to observing groups in action.

Though the longitudinal design utilized in the current study is recognized as a strength of this study, it is limited, as noted below, in that it has only captured a relatively short lapse in time. This fifth important limitation of the current study may have an effect on all hypotheses that predicted change over time. Specifically, the time lapse between measurements is approximately $10-12$ weeks. Due to the lack of understanding of the dynamic nature of masculinity ideology, it is unclear whether this short duration of time is enough to capture change in adherence to masculinity ideology over time.

A sixth limitation of the current study concerns the concept of model specification error (e.g., Kline, 2010). Though important individual and contextual 
variable influences on the development of masculinity ideology have been assessed, other potentially important variables have been left out. In particular, disability status is an important individual variable to consider in future research with youth in juvenile justice facilities as the prevalence of disabilities among youth in the juvenile justice system is high. For example, whereas prevalence of disabilities among school age children in the United States $(9 \%)$ is much lower than the conservative estimate of those in the juvenile justice system (32\%) (Quinn, Rutherford, \& Leone, 2001). Similar to race/ethnicity, social class, or sexual orientation, men with disabilities may also experience difficulties in gaining access to dominant and traditional ideals of masculinity (Gerschick \& Miller, 1997). Perhaps as a result of the "blocked" access, men with disabilities have described their own alternative forms of masculinity (Gerschick \& Miller). Future research should consider the influence of disability status on masculinity ideology development.

A final limitation of the study concerns the amount of missing data and its potential influence on the findings. Of particular concern is how youth who stayed in the study over the course of the approximately 20 -weeks differed from those who dropped out. In particular, if youth dropped out of the study because they were released from the juvenile justice facility, they may differ from the youth who remain. One difference may be by age, older youth move onto adult prison or by the severity of the felony charge. On the other hand, there is also concern that those who entered the study after baseline differed from those who completed baseline measurements. To better understand this potential impact, analyses were conducted 
on the predicted missingness of each variable at one survey measurement occasion during the other occasions of measurement. In short, whether or not a participant was missing at a specific measurement occasion sometimes influenced the responses at other occasions. For example, age at the third survey occasion was predicted by missingness on the variable age at baseline. Specifically, youth who completed a baseline measurement tended to be younger at the third measurement occasion compared to those who were missing at baseline. To be clear, at the third measurement occasion, youth who were older were less likely to have completed a baseline measurement than those who were younger. This effect was detected for youth missing a second survey occasion, but reversed for those missing survey occasions 4 and 5 . In other words, older youth at time 3 were more likely to have completed a baseline and occasion 2 measurements and less likely to have completed occasions 4 and 5. This makes sense given the nature of the environment in which the study took place, in that youth are released or sent to adult prison by the time they turn 21 years of age. For these reasons, the study is limited in that the patterning of completing survey measurements (timing of entering into and/or dropping out of the study) is sometimes related to participant demographic, behavioral, and attitudinal characteristics.

This limitation may have influenced the finding of hypothesis $1 \mathrm{~b}$, that youth who attended more hours of The Council tended to have higher initial levels of adherence to traditional masculinity ideology. Clearly, the initial level of adherence cannot be affected by participation in The Council as this was assessed before the 
youth had attended a group. There appears to be a selection bias in the implementation of The Council in ODYS, in that youth with higher initial levels of adherence to traditional masculinity ideology were more likely to attend a greater number of Council groups. The underlying cause of this relationship is unclear, perhaps youth with high levels of adherence are self-selecting to attend a greater number of group sessions. Regardless of the reason, from a practitioner's perspective, the youth with highest levels of traditional masculinity ideology may be in greatest need of this group. On these grounds, it may be seen as a good sign that these youth are attending the greatest number of hours. In addition, the patterning of attendance was not assessed in this study. Thus, it is unclear whether the timing of the sessions attended (e.g., first 5 sessions vs. last 5 sessions) has an effect on the outcomes.

\section{Strengths}

Despite the above-mentioned limitations, the current study makes several meaningful contributions to the literature on masculinity ideology in adolescent young men. Perhaps the most significant strength of the current study is the use of longitudinal design and analytic method to assess changes in adherence to traditional masculinity ideology over time. To my knowledge, this is the first study to assess change in adherence to masculinity ideology over time for a sample of adolescent incarcerated males. Thus, the study provides some insight into the apparent dynamic nature of masculinity ideology during an age of important physical, social, and individual development and within a context in which the study of male gender is 
desperately needed. For example, the significant finding that youth of different racial/ethnic backgrounds (African American and White) and youth with differing levels of ethnic pride within a racial/ethnic group (African American) have different trajectories of change in levels of traditional masculinity ideology over the course of the study provides a better understanding of how multiple dimensions of diversity interact with each other and masculinity ideologies over time.

A second notable strength of this study is its use of multiple sources of data. Qualitative researchers (Merrick, 1999; Lincoln \& Guba, 1985) have argued for a triangulation of methodologies such that one construct is assessed using multiple forms of data collection. The rationale behind the advocacy of triangulation is that it provides a more thorough and complete understanding of the construct. The current study uses responses to paper-and-pencil surveys - both open-ended, qualitative responses as well as closed-ended quantitative responses - and facility records data. In this way, masculinity and changes in masculinity that may be caused by participation in The Council were assessed using both a closed-ended survey and open-ended questions regarding what youth learned about being male. As is described above, the open-ended responses partly overlapped with the closed-ended responses, but also filled in some gaps in the short 12-item measure. Additionally, important demographic predictor variables were assessed via self-reports and verified using facility records data. Therefore, most of the study's constructs were assessed with more than one measure, which increases the validity of these constructs and confidence in the results. 


\section{Implications and Future Directions}

The dissertation study examined how several contextual variables might influence one's adherence to traditional masculinity ideology. Given the literature that has linked high levels of adherence to traditional masculinity with problem behaviors among adolescent populations (e.g., Pleck, Sonenstein, \& Ku, 1993), understanding the antecedents of change in adherence is both theoretically and practically important.

One important contribution made by the study is the finding that level of adherence to traditional masculinity ideology may be affected by participation in a strength-based, gender focused program. Specifically, adolescent inmates in the study who participated in a greater amount of The Council sessions adhered less strongly to traditional masculinity ideology over time compared to youth who attended fewer sessions. Considering the context of the juvenile justice system and the established association between high levels of adherence to traditional masculinity ideology and problem behaviors, having an effect on changing trajectories of development of traditional masculinity ideology may have important implications on the experiences of the youth while in prison. For example, youth who adhere less strong or rigidly to traditional masculinity ideology may be more likely to exhibit behaviors that are acceptable within the system and that may be recognized as good behavior that warrants reward or earlier release. In other words, it is implied that high and rigid levels of adherence to traditional masculinity ideology is not adaptive for youth in juvenile justice facilities and therefore, the 
finding that level of adherence can be influenced by programming implemented within the facility suggest the need to continue implementing this program.

Though it is implied, this dissertation does not consider whether or how adherence to traditional masculinity ideology is directly related to success or failure within juvenile justice facilities. In fact, the quantitative measurement of adherence to traditional masculinity ideology (AMIRS; Chu et al., 2005) takes a normative perspective that assesses youth's internalization of traditional masculinity. And, although this measure has been shown to be correlated with lower levels of selfesteem and a greater likelihood of demonstrating aggressive and deviant behaviors (Chu et al.), scores on this scale have not been examined in relation to actual observed behavior. In other words, it is unclear whether youth's internalization of traditional masculinity is manifested through physical and social behavior. For example, given the descriptions of the "masks" of masculinity (e.g., Pollack, 1996) previously described, youth with low levels of adherence to traditional masculinity ideology may still demonstrate behaviors that are characterized as highly traditional and vice versa. Therefore, because the current study does not model behavior and because the relationship between identity and behaviors related to traditional masculinity are not always clear, results from this study cannot be used to directly predict behavior within the juvenile justice system. Moreover, this dissertation does not consider the long-term effects of changing levels of adherence to traditional masculinity ideology for youth who are released from the juvenile justice system to their communities or to the adult prison system. Future studies should extend 
examination of traditional masculinity ideology development among adolescent inmates and follow these youth through their release from the system. Moreover, future studies should assess whether and how traditional masculinity ideology is adaptive or maladaptive both within the juvenile justice system and outside. Ideally, future research would bridge a gap that exists in the current study between the normative assessment of adherence to traditional masculinity ideology and actual observed behaviors.

A second important implication of the current study is that youth of different ages, different race/ethnicity (African American or White), varying levels of ethnic belonging among African American men, and amount of participation in The Council adhere more or less strongly to traditional masculinity ideology over time. This finding provides support for the theory of multiple masculinities or the idea that one's masculinity ideology is developed, maintained, and restructured according to one's social and environmental contexts (e.g., Smiler, 2004). As was reviewed in Chapter II, the study of masculinity has taken multiple forms over the years. Most recently, the deconstructionist movement has described masculinity ideology to be externally and socially defined and something that may be altered by the social setting or context in which an individual resides (Smiler). The study finding illustrate how traditional masculinity ideology takes on different forms for individuals of different groups differently over time. In particular, results from the current study add to important conversations regarding the dynamic nature of masculinity ideology development and race/ethnic group differences. The next step would be to extend 
this study longitudinally so that within individual changes over time and developmental age may be determined. Additionally, within race/ethnic group variation in relation to masculinity ideology development must be further explored in order to explain why group level differences have been detected and what the implications of these group level differences are.

Finally, this dissertation contributes to the sparse literature examining masculinity ideology development among a sample of incarcerated adolescents. Through the use of a qualitative follow-up research design sequence, responses to open-ended questions regarding youth's experiences being male and participating in a gender specific strength-based program, helped illuminate gaps in quantitative assessment of traditional masculinity ideology. There are several explanations for why gaps in measurement were identified in the current study. For example, Chu and colleagues (2005) used a sample of predominantly White (62-79\%) (compared to only 0-9\% who identified as African American) seventh and eighth grade and high school boys to assess the validity and reliability of the scale. Though socioeconomic status was not reported in that study, 36-41\% of the participants' mothers completed at least some college. In the current study, however, the majority of the sample identified as African American and all were incarcerated in the juvenile justice system. Results regarding the moderating effect of ethnic pride for African American participants in combination with some of the gaps in measurement identified through the qualitative analysis bring into question the validity of the Adolescent Masculinity Ideology in Relationships Scale (AMIRS; Chu et al.) for African American youth. In 
addition, qualitative findings may bring into question the validity of the use of this scale for a population of adolescent inmates. To review, the AMIRS (Chu et al., 2005) was developed to measure masculinity ideology within the context of boys' interpersonal relationships. Interpersonal interactions among youth in school settings are limited in duration (during the school day), but are varied in context (e.g., classroom vs. recess) and in group composition. Though youth are likely to experience greater interactions with their classmates, they also have lunch and recess with peers from different classrooms, which allows for free and unstructured "play" time with these peers. On the other hand, interpersonal interactions in the juvenile justice system are typically more constant (e.g., cell mates, group programs, showers, eating) and interpersonal interactions are closely observed and may be restricted. Given interpersonal relationships among youth in school settings are different from those in the juvenile justice system and the questionable validity among African American participants, the factor structure and the validity the AMIRS within juvenile justice systems should be assessed in future research.

One example of the gaps in content of masculinity ideology that were identified in the quantitative measure is captured in the qualitative theme "Man Up". Specifically, youth described a form of masculinity ideology referred to in the study and by the youth as "Man Up". This alternative form of masculinity parallels many of the concepts of traditional masculinity, but provides an additional level of flexibility that has been denied in the traditional form. Future studies should continue to assess new forms of masculinity ideology among diverse samples of boys and men 
in order to understand how contemporary forms of masculinity ideology differ from traditional forms and whether these contemporary forms are related to the same set of poor health and behavioral outcomes. 


\section{ENDNOTES}

${ }^{1}$ The term "storm and stress" was first coined by a German play-write, Friedrich Maximiliam Klinger, in 1776 through use of the German term "sturm und drang" (literally translated to English as "storm and urge", but usually translated as "storm and stress").

${ }^{2}$ The number of inmates detained at the four study sites within ODYS during the time of the study was estimated using the Records from ODYS. Specifically, the number of individuals with records during the third measurement (all sites included) was used as an estimate of the number of individuals at the four locations during the time of the study.

${ }^{3}$ The Urban Dictionary provides definitions to slang and ethnic culture words and phrases that are not common in standard dictionaries. 
Table 1. Masculinity Research Eras

\begin{tabular}{|c|c|c|}
\hline Era & Characteristics & $\begin{array}{l}\text { Healthy/Unhealthy } \\
\text { Masculinity }\end{array}$ \\
\hline $\begin{array}{l}\text { Sex-Role } \\
\text { Identity (e.g., } \\
\text { Brown, 1958; } \\
\text { Guilford \& } \\
\text { Zimmerman, 1956; } \\
\text { Terman \& Miles, } \\
\text { 1936) }\end{array}$ & $\begin{array}{l}\text { - Single-dimension of } \\
\text { masculinity opposed } \\
\text { femininity } \\
\text { - Inherent }\end{array}$ & $\begin{array}{l}\text { Individual's identified sex } \\
\text { matched their preference = } \\
\text { healthy } \\
\text { - } \begin{array}{l}\text { High levels of masculine } \\
\text { characteristics = healthy }\end{array}\end{array}$ \\
\hline $\begin{array}{l}\text { Androgyny } \\
(\mathrm{Bem}, 1974)\end{array}$ & $\begin{array}{ll}\text { - } & \text { Multi-dimensional } \\
\text { - } & \text { Inherent } \\
\text { - } & \text { Individual can } \\
& \text { possess both masc } \\
\text { and fem traits }\end{array}$ & $\begin{array}{l}\text { Sex-role flexibility (a.k.a } \\
\text { androgyny) = healthy }\end{array}$ \\
\hline $\begin{array}{l}\text { Masculinity } \\
\text { Ideology (e.g., } \\
\text { Brannon, 1985) }\end{array}$ & $\begin{array}{l}\text { - Gender as a cultural } \\
\text { and social ideal in } \\
\text { which individuals } \\
\text { attempt to conform }\end{array}$ & $\begin{array}{l}\text { Traditional Masculine Ideal = } \\
\text { - } \text { Anti-femininity } \\
\text { - } \text { Status \& Achievement } \\
\text { - Inexpressiveness \& } \\
\text { - } \text { Independence } \\
\text { Adventurous and } \\
\text { Aggressive } \\
\text { Adherence to traditional masculine } \\
\text { ideals = unhealthy }\end{array}$ \\
\hline $\begin{array}{l}\text { Gender role } \\
\text { conflict/strain/s } \\
\text { tress (e.g., Eisler } \\
\text { \& Skidmore, 1987; } \\
\text { Garnet \& Pleck, } \\
\text { 1979; O'Neil et al., } \\
\text { 1981) }\end{array}$ & $\begin{array}{l}\text { Considers } \\
\text { discrepancy between } \\
\text { one's real gender and } \\
\text { cultural gender ideal. } \\
\text { - Considers negative } \\
\text { consequences of } \\
\text { attaining traditional } \\
\text { masculine ideal }\end{array}$ & $\begin{array}{ll} & \text { Rigid and restrictive } \\
\text { masculine gender role } \\
\text { norms = unhealthy }\end{array}$ \\
\hline $\begin{array}{l}\text { Deconstructioni } \\
\text { st Masculinity }\end{array}$ & $\begin{array}{ll}\text { - } & \text { Gender is socially } \\
\text { defined } \\
\text { - } & \text { Altered by social } \\
\text { setting } \\
\text { - } & \text { Multiple } \\
\text { masculinities }\end{array}$ & $\begin{array}{l}\text { No specific form or level } \\
\text { of masculinity is specific } \\
\text { or ideal }\end{array}$ \\
\hline
\end{tabular}


Table 2. The Council Curricula: Themes and Activities

\begin{tabular}{|c|c|c|c|}
\hline & $\begin{array}{c}\text { Standing } \\
\text { Together: A } \\
\text { Boys Council } \\
\text { Journey into }\end{array}$ & $\begin{array}{c}\text { Growing Healthy, } \\
\text { Growing Strong }\end{array}$ & $\begin{array}{c}\text { Living a } \\
\text { Legacy: A Boys } \\
\text { Council Rite of } \\
\text { Passage }\end{array}$ \\
\hline Week 1 & $\begin{array}{l}\text { Creating Our } \\
\text { Council }\end{array}$ & $\begin{array}{l}\text { Creating Our } \\
\text { Council }\end{array}$ & $\begin{array}{l}\text { Creating Our } \\
\text { Council }\end{array}$ \\
\hline Week 2 & $\begin{array}{l}\text { Similar and } \\
\text { Different }\end{array}$ & Getting Connected & $\begin{array}{l}\text { Creating Our } \\
\text { Council }\end{array}$ \\
\hline Week 3 & $\begin{array}{c}\text { Put Downs - Part } \\
1\end{array}$ & $\begin{array}{c}\text { Healthy } \\
\text { Competition - Part } \\
1\end{array}$ & $\begin{array}{l}\text { Strength } \\
\text { Through } \\
\text { Diversity }\end{array}$ \\
\hline Week 4 & $\begin{array}{c}\text { Put Downs - Part } \\
2\end{array}$ & $\begin{array}{c}\text { Healthy } \\
\text { Competition - Part } \\
2\end{array}$ & $\begin{array}{l}\text { Mentors, Role } \\
\text { Models, and } \\
\text { Heroes }\end{array}$ \\
\hline Week 5 & Space Invaders & Bullying & $\begin{array}{l}\text { Unlocking the } \\
\text { Code }\end{array}$ \\
\hline Week 6 & Boys' Rights & $\begin{array}{c}\text { What's Your } \\
\text { Choice? Boys and } \\
\text { Their Emotions - } \\
\text { Part } 1\end{array}$ & $\begin{array}{c}\text { Healthy } \\
\text { Relationships }\end{array}$ \\
\hline Week 7 & $\begin{array}{c}\text { E-motions - Part } \\
1\end{array}$ & $\begin{array}{c}\text { What's Your } \\
\text { Choice? Boys and } \\
\text { Their Emotions - } \\
\text { Part } 2\end{array}$ & $\begin{array}{c}\text { Who's the Man? } \\
\text { Boys and the } \\
\text { Media }\end{array}$ \\
\hline Week 8 & $\begin{array}{c}\text { E-motions - Part } \\
2\end{array}$ & $\begin{array}{c}\text { Boys' Unspoken } \\
\text { Rules }\end{array}$ & $\begin{array}{c}\text { Conflict } \\
\text { Resolution: } \\
\text { Squash it Before } \\
\text { it Starts }\end{array}$ \\
\hline Week 9 & Boys \& Power & $\begin{array}{c}\text { Male \& Female: } \\
\text { Roles and } \\
\text { Expectations }\end{array}$ & $\begin{array}{c}\text { No One Walks } \\
\text { Alone }\end{array}$ \\
\hline Week 10 & $\begin{array}{l}\text { Community \& } \\
\text { Recognition }\end{array}$ & Staying Connected & $\begin{array}{l}\text { Living and } \\
\text { Leaving a } \\
\text { Legacy }\end{array}$ \\
\hline
\end{tabular}


Table 3. Participants Demographic Information by location

\begin{tabular}{|c|c|c|c|c|c|c|}
\hline & & ORV & Circleville & IR & $\mathrm{CH}$ & \\
\hline Age & & & & & & Total \\
\hline & 12 years & 0 & 0 & 0 & 1 & $1(<1 \%)$ \\
\hline & 13 years & 0 & 0 & 1 & 10 & $11(<1 \%)$ \\
\hline & 14 years & 4 & 2 & 8 & 22 & $36(3 \%)$ \\
\hline & 15 years & 40 & 10 & 57 & 49 & $\begin{array}{c}156 \\
(13 \%)\end{array}$ \\
\hline & 16 years & 70 & 42 & 94 & 94 & $\begin{array}{c}300 \\
(24 \%)\end{array}$ \\
\hline & 17 years & 106 & 46 & 127 & 88 & $\begin{array}{c}367 \\
(29 \%)\end{array}$ \\
\hline & 18 years & 69 & 30 & 72 & 58 & $\begin{array}{c}229 \\
(18 \%)\end{array}$ \\
\hline & 19 years & 30 & 20 & 38 & 11 & $99(8 \%)$ \\
\hline & 20 years & 22 & 7 & 9 & 6 & $44(4 \%)$ \\
\hline & missing & 0 & 0 & 3 & 2 & $5(<1 \%)$ \\
\hline & Total & 341 & 157 & 409 & 341 & \\
\hline \multicolumn{7}{|l|}{$\begin{array}{l}\text { Ethnic } \\
\text { Identity }\end{array}$} \\
\hline & White & 47 & 36 & 148 & 79 & $\begin{array}{c}310 \\
(25 \%)\end{array}$ \\
\hline & Asian & 0 & 1 & 2 & 0 & $3(<1 \%)$ \\
\hline & Latino & 9 & 4 & 9 & 7 & $29(2 \%)$ \\
\hline & $\begin{array}{r}\text { Native } \\
\text { American }\end{array}$ & 3 & 3 & 5 & 3 & $14(1 \%)$ \\
\hline & $\begin{array}{r}\text { African } \\
\text { American }\end{array}$ & 265 & 103 & 216 & 226 & $\begin{array}{c}810 \\
(65 \%)\end{array}$ \\
\hline & Other & 6 & 6 & 8 & 13 & $33(3 \%)$ \\
\hline & Multiple & 10 & 4 & 21 & 11 & $46(4 \%)$ \\
\hline & missing/unknown & 1 & & & 2 & $3(<1 \%)$ \\
\hline & Total & 341 & 157 & 409 & 341 & $N=1248$ \\
\hline
\end{tabular}


Table 4. Patterning distribution of participants survey responses $(\mathrm{n}=1248)$

\begin{tabular}{|l|r|r|r|r|r|r|r|r|r|}
\hline & $\begin{array}{c}\text { T1 } \\
\text { only }\end{array}$ & \multicolumn{1}{|c|}{$\begin{array}{c}\text { T2 } \\
\text { only }\end{array}$} & \multicolumn{1}{|c|}{$\begin{array}{c}\text { T3 } \\
\text { only }\end{array}$} & $\begin{array}{r}\text { T1 \& } \\
\text { T2 }\end{array}$ & \multicolumn{1}{c|}{$\begin{array}{c}\text { T1 \& } \\
\text { T3 }\end{array}$} & $\begin{array}{c}\text { T2 \& } \\
\text { T3 }\end{array}$ & $\begin{array}{c}\text { All } \\
\text { three }\end{array}$ & $\begin{array}{c}\text { Total } \\
\text { N }\end{array}$ & $\begin{array}{r}\text { Percent of } \\
\text { total } \\
\text { sample }\end{array}$ \\
\hline ORV & 64 & 59 & 64 & 49 & 12 & 44 & 49 & 341 & $27 \%$ \\
\hline Circleville & 35 & 37 & 35 & 19 & 3 & 18 & 10 & 157 & $13 \%$ \\
\hline CH & 100 & 54 & 98 & 34 & 11 & 47 & 65 & 409 & $33 \%$ \\
\hline IR & 78 & 43 & 63 & 40 & 12 & 39 & 66 & 341 & $27 \%$ \\
\hline
\end{tabular}


Table 5. Frequency count of attendance in hours

\begin{tabular}{|c|c|c|c|c|}
\hline & \multicolumn{2}{|c|}{ Ohio River Valley } & \multicolumn{2}{|c|}{ Circleville } \\
\hline & First $10-w k s$ & Second $10-$ wks & First 10-wks & Second 10-wks \\
\hline $0 \mathrm{hrs}$ & 24 & 8 & 8 & 5 \\
\hline $1 \mathrm{hr}$ & 0 & 0 & 7 & 2 \\
\hline $2 \mathrm{hrs}$ & 4 & 2 & 3 & 2 \\
\hline $3 \mathrm{hrs}$ & 0 & 1 & 1 & 1 \\
\hline $4 \mathrm{hrs}$ & 5 & 3 & 0 & 0 \\
\hline $5 \mathrm{hrs}$ & 1 & 0 & 2 & 1 \\
\hline $6 \mathrm{hrs}$ & 8 & 4 & 2 & 4 \\
\hline $7 \mathrm{hrs}$ & 4 & 15 & 2 & 1 \\
\hline $8 \mathrm{hrs}$ & 4 & 3 & 6 & 3 \\
\hline $9 \mathrm{hrs}$ & 4 & 5 & 4 & 4 \\
\hline $10 \mathrm{hrs}$ & 9 & 6 & 1 & 1 \\
\hline $11 \mathrm{hrs}$ & 2 & 1 & 2 & 3 \\
\hline $12 \mathrm{hrs}$ & 7 & 3 & 0 & 3 \\
\hline $13 \mathrm{hrs}$ & 2 & 4 & 1 & 1 \\
\hline $14 \mathrm{hrs}$ & 5 & 7 & 2 & 0 \\
\hline $15 \mathrm{hrs}$ & 0 & 7 & 2 & 2 \\
\hline $16 \mathrm{hrs}$ & 17 & 19 & 2 & 3 \\
\hline $17 \mathrm{hrs}$ & 8 & 16 & 4 & 2 \\
\hline $18 \mathrm{hrs}$ & 20 & 8 & 7 & 5 \\
\hline $19 \mathrm{hrs}$ & 6 & 4 & 2 & 1 \\
\hline $20 \mathrm{hrs}$ & 43 & 8 & 3 & 2 \\
\hline$>20 \mathrm{hrs}$ & 8 & 2 & 7 & 8 \\
\hline missing & 160 & 215 & 89 & 103 \\
\hline Total & 341 & 341 & 157 & 157 \\
\hline
\end{tabular}


Table 6a. Complete Research Design

\begin{tabular}{|c|c|c|c|c|c|}
\hline $\begin{array}{c}\text { ODYS Study } \\
\text { Partners }\end{array}$ & $\begin{array}{c}\text { Survey } \\
1 \\
\\
\text { June } \\
2009 \\
\end{array}$ & $\begin{array}{c}\text { Survey } 2 \\
\text { Aug-Sep } \\
2009\end{array}$ & $\begin{array}{c}\text { Survey } \\
3 \\
\\
\text { Nov } \\
2009 \\
\end{array}$ & $\begin{array}{c}\text { Survey } \\
4 \\
\text { Feb } 2010\end{array}$ & $\begin{array}{c}\text { Survey } 5 \\
\text { Apr-May } \\
2010\end{array}$ \\
\hline Circleville & $\mathbf{X}_{\mathbf{B}}$ & $\mathbf{X}_{\mathbf{B}}$ & $\mathbf{X}_{\mathbf{B}}$ & $\mathbf{X}_{\mathbf{T}}$ & $\mathbf{X}_{\mathbf{T}}$ \\
\hline Cuyahoga Hills & & & $\mathbf{X}_{\mathbf{C}}$ & $\mathbf{X}_{\mathbf{C}}$ & $\mathbf{X}_{\mathbf{C}}$ \\
\hline Indian River & & & $\mathbf{X}_{\mathbf{C}}$ & $\mathbf{X}_{\mathbf{C}}$ & $\mathbf{X}_{\mathbf{C}}$ \\
\hline Ohio River Valley & $\mathbf{X}_{\mathbf{C}}$ & $\mathbf{X}_{\mathbf{T}}$ & $\mathbf{X}_{\mathbf{T}}$ & $\mathbf{X}_{\mathbf{T}}$ & $\mathbf{X}_{\mathrm{T}}$ \\
\hline
\end{tabular}

Key:

$$
\begin{aligned}
& \mathrm{X}_{\mathrm{T}}=\text { Treatment group }=\text { Received The Council } \\
& \mathrm{X}_{\mathrm{C}}=\text { Control group } \\
& \mathrm{X}_{\mathrm{B}}=\text { Baseline }
\end{aligned}
$$

Table 6b. Research Design of Proposed Dissertation

\begin{tabular}{|c|c|c|c|}
\hline $\begin{array}{c}\text { ODYS Study } \\
\text { Partners }\end{array}$ & Survey 1 & Survey 2 & Survey 3 \\
\hline Circleville & $\mathrm{X}_{\mathrm{B}}$ & $\mathrm{X}_{\mathrm{T}}$ & $\mathrm{X}_{\mathrm{T}}$ \\
\hline Cuyahoga Hills & $\mathrm{X}_{\mathrm{C}}$ & $\mathrm{X}_{\mathrm{C}}$ & $\mathrm{X}_{\mathrm{C}}$ \\
\hline Indian River & $\mathrm{X}_{\mathrm{C}}$ & $\mathrm{X}_{\mathrm{C}}$ & $\mathrm{X}_{\mathrm{C}}$ \\
\hline Ohio River Valley & $\mathrm{X}_{\mathrm{B}}$ & $\mathrm{X}_{\mathrm{T}}$ & $\mathrm{X}_{\mathrm{T}}$ \\
\hline
\end{tabular}

Note: Greyed out measurements from table above are removed for purposes of proposed study Key:

$\mathrm{X}_{\mathrm{T}}=$ Treatment group $=$ Received The Council

$\mathrm{X}_{\mathrm{B}}=$ Baseline measure before the introduction of The Council

$\mathrm{X}_{\mathrm{C}}=$ Control group 
Table 7a. Descriptive statistics of continuous study variables

\begin{tabular}{|c|c|c|c|c|c|}
\hline \multicolumn{6}{|c|}{ Control Locations: Cuyahoga Hills \& Indian River } \\
\hline & $\mathrm{N}$ & Min & Max & Mean & $\begin{array}{l}\text { Stand. } \\
\text { Dev. }\end{array}$ \\
\hline $\mathrm{Age}^{\mathrm{I}}$ & 745 & 12 & 20 & 16.64 & 1.40 \\
\hline Days in Prison & 592 & 0 & 1932 & 380.72 & 289.52 \\
\hline $\begin{array}{c}\text { Attendance }^{2} \\
\text { First } 10 \text { weeks }\end{array}$ & 1 & 10 & 10 & 10.0 & .0 \\
\hline $\begin{array}{c}\text { Attendance } \\
\text { Second } 10 \text { weeks }\end{array}$ & 2 & 1 & 1 & 1 & .0 \\
\hline AMIRS - Survey 1 & 404 & 1 & 3.58 & 2.15 & .447 \\
\hline AMIRS - Survey 2 & 385 & 1 & 3.42 & 2.17 & .445 \\
\hline AMIRS - Survey 3 & 397 & 1 & 3.58 & 2.16 & .453 \\
\hline Ethnic Pride & 742 & 0 & 4.0 & 3.00 & 1.27 \\
\hline \multicolumn{6}{|c|}{ Experimental Locations: Circleville \& Ohio River Valley } \\
\hline & $\mathrm{N}$ & Min & Max & Mean & $\begin{array}{l}\text { Stand. } \\
\text { Dev. }\end{array}$ \\
\hline $\mathrm{Age}^{\mathrm{I}}$ & 498 & 14 & 20 & 17.11 & 1.36 \\
\hline Days in Prison & 284 & 4 & 1928 & 664.96 & 383.84 \\
\hline $\begin{array}{c}\text { Attendance }^{2} \\
\text { First } 10 \text { weeks }\end{array}$ & 218 & 1 & 23 & 14.26 & 6.11 \\
\hline $\begin{array}{c}\text { Attendance }^{2} \\
\text { Second } 10 \text { weeks }\end{array}$ & 169 & 1 & 22 & 13.21 & 5.40 \\
\hline AMIRS - Survey 1 & 240 & 1.17 & 3.50 & 2.26 & .414 \\
\hline AMIRS - Survey 2 & 284 & 1.0 & 3.42 & 2.29 & .370 \\
\hline AMIRS - Survey 3 & 232 & 1.17 & 3.50 & 2.34 & .353 \\
\hline Ethnic Pride & 487 & 0 & 4.0 & 3.00 & 1.28 \\
\hline
\end{tabular}

Notes:

${ }^{1}$ The distribution of participant's age is described in greater detail in Table 3. 
${ }^{2}$ The distribution of participant's attendance in The Council is described in greater detail in Table 5. Attendance described above only for participants with 1 hour or greater reported.

${ }^{3}$ Ethnic identity is described only in Table 3. 
Table $7 b$. Descriptive statistics of continuous study variables

\begin{tabular}{|l|c|c|c|c|c|c|}
\hline \multicolumn{7}{|c|}{ Complete Study Participants (n=1248) } \\
\hline & $\mathrm{N}$ & Min & Max & Mean & $\begin{array}{c}\text { Stand. } \\
\text { Dev. }\end{array}$ & $\begin{array}{c}\text { Cronbach's } \\
\text { Alpha }\end{array}$ \\
\hline Age $^{1}$ & 1243 & 12 & 20 & 16.83 & 1.40 & \\
\hline Days in Prison & 876 & 0 & 1932 & 472.87 & 349.27 & \\
\hline $\begin{array}{l}\text { Attendance } \\
\text { First 10 weeks }\end{array}$ & 218 & 1 & 23 & 14.24 & 6.10 & \\
\hline $\begin{array}{l}\text { Attendance } \\
\text { Second 10 weeks }\end{array}$ & 169 & 1 & 22 & 13.07 & 5.53 & \\
\hline AMIRS - Survey 1 & 644 & 1 & 3.58 & 2.19 & .438 & .744 \\
\hline AMIRS - Survey 2 & 669 & 1 & 3.42 & 2.22 & .419 & .723 \\
\hline AMIRS - Survey 3 & 629 & 1 & 3.58 & 2.23 & .427 & .727 \\
\hline Ethnic Pride & 1229 & 0 & 4.0 & 3.00 & 1.27 & \\
\hline
\end{tabular}

Notes:

${ }^{1}$ The distribution of participant's age is described in greater detail in Table 3.

${ }^{2}$ The distribution of participant's attendance in The Council is described in greater detail in Table 5. Attendance described above only for participants with 1 hour or greater reported.

${ }^{3}$ Ethnic identity is described only in Table 3. 
Table 8. Time of measurement non-response

\begin{tabular}{|c|c|c|c|c|c|c|}
\hline \multicolumn{7}{|c|}{ Survey } \\
\hline & $\mathrm{T} 1$ & $\mathrm{~T} 2$ & T3 & $\mathrm{T} 4$ & T5 & Sum \\
\hline & June 2009 & $\begin{array}{c}\text { Aug- } \\
\text { Sep } \\
2009 \\
\end{array}$ & $\begin{array}{l}\text { Nov } \\
2009\end{array}$ & $\begin{array}{l}\text { Feb } \\
2010\end{array}$ & $\begin{array}{l}\text { Apr- } \\
\text { Mar } \\
2010 \\
\end{array}$ & \\
\hline Ohio River Valley & 181 & 227 & 181 & 157 & 133 & \\
\hline \multirow[t]{2}{*}{ Circleville } & 116 & 82 & 77 & 91 & 64 & \\
\hline & Nov 2009 & $\begin{array}{l}\text { Feb } \\
2010\end{array}$ & $\begin{array}{l}\text { Apr- } \\
\text { Mar } \\
2010 \\
\end{array}$ & & & \\
\hline Indian River & 215 & 197 & 186 & 16 & 11 & \\
\hline Cuyahoga Hills & 214 & 207 & 231 & 5 & 12 & \\
\hline $\begin{array}{c}\text { Unknown location (- } \\
99)\end{array}$ & 1 & 5 & 8 & 2 & 5 & \\
\hline $\begin{array}{l}\text { Number of youth } \\
\text { who completed a } \\
\text { survey at the } \\
\text { specified time - } \\
\text { TOTAL } \\
\end{array}$ & 727 & 718 & 683 & 271 & 225 & 2624 \\
\hline $\begin{array}{c}\text { Approximate } \\
\text { number of youth } \\
\text { detained in ODYS at } \\
\text { any given time } \\
\text { during 2009-2010 }\end{array}$ & 1,077 & 1,077 & 1,077 & 1,077 & 1,077 & 5,385 \\
\hline Percent completed & $67.50 \%$ & $66.67 \%$ & $63.42 \%$ & $25.16 \%$ & $20.89 \%$ & $48.73 \%$ \\
\hline
\end{tabular}


Table 9a. Predicted missingness of age: Missingness at four survey time points regressed on age at the fifth survey time

\begin{tabular}{|c|c|c|c|c|c|c|}
\hline & \multicolumn{5}{|c|}{ Missing data ( $0=$ missing; 1 = present $)$} & \\
\hline & T1 & $\mathrm{T} 2$ & T3 & T4 & T5 & \\
\hline $\begin{array}{l}\text { Age at T1- } \\
\text { survey (valid } \\
\text { response) }\end{array}$ & & $\begin{array}{l}b=- \\
.082, S E \\
=.12, \beta \\
=-.028\end{array}$ & $\begin{array}{l}\mathrm{b}=- \\
.254, \mathrm{SE} \\
=.127, \beta \\
=-.084^{*}\end{array}$ & $\begin{array}{l}b=.134, \\
S E= \\
.188, \beta= \\
.032\end{array}$ & $\begin{array}{l}b=-.244, \\
S E=.197, \\
\beta=-.054\end{array}$ & $R^{2}=.011$ \\
\hline $\begin{array}{l}\text { Age at T2- } \\
\text { survey (valid } \\
\text { response) }\end{array}$ & $\begin{array}{l}b=.336 \\
S E=.106, \\
\beta= \\
.118^{* *}\end{array}$ & & $\begin{array}{l}b=- \\
.321, \mathrm{SE} \\
=.108, \beta \\
=-.11^{*}\end{array}$ & $\begin{array}{l}b=.305, \\
S E= \\
.178, \beta= \\
.077\end{array}$ & $\begin{array}{l}b=-.163, \\
S E=.197, \\
\beta=-.037\end{array}$ & $\begin{array}{l}\mathrm{R}^{2}= \\
.026 * *\end{array}$ \\
\hline $\begin{array}{l}\text { Age at T3- } \\
\text { survey (valid } \\
\text { response) }\end{array}$ & $\begin{array}{l}b=.402, \\
S E=.119, \\
\beta=.14^{* *}\end{array}$ & $\begin{array}{l}b= \\
.085, S E \\
=.116 \\
\beta=.03\end{array}$ & & $\begin{array}{l}b=.407, \\
S E= \\
.165, \beta= \\
.115^{*}\end{array}$ & $\begin{array}{l}b=-.06, \\
S E=.195, \\
\beta=-.014\end{array}$ & $\begin{array}{l}\mathrm{R}^{2}= \\
.037 * * *\end{array}$ \\
\hline $\begin{array}{l}\text { Age at T4- } \\
\text { survey (valid } \\
\text { response) }\end{array}$ & $\begin{array}{l}b=.262, \\
S E=.166, \\
\beta=.097\end{array}$ & $\begin{array}{l}b=.22, \\
S E= \\
.179, \beta \\
=.083\end{array}$ & $\begin{array}{l}b=.358, \\
S E= \\
.173, \beta= \\
.137^{*}\end{array}$ & & $\begin{array}{l}b=-.468, \\
S E=.158, \\
\beta=- \\
.179 * *\end{array}$ & $\begin{array}{l}\mathrm{R}^{2}= \\
.072 * *\end{array}$ \\
\hline $\begin{array}{l}\text { Age at T5- } \\
\text { survey (valid } \\
\text { response) }\end{array}$ & $\begin{array}{l}b=.271, \\
S E=.184, \\
\beta=.102\end{array}$ & $\begin{array}{l}b= \\
.026, S E \\
=.202, \\
\beta=.01\end{array}$ & $\begin{array}{l}b=.489, \\
S E= \\
.209, \beta= \\
.185^{*}\end{array}$ & $\begin{array}{l}\mathrm{b}=- \\
.377, \mathrm{SE} \\
=.188, \beta \\
=-.145^{*}\end{array}$ & & $\begin{array}{l}\mathrm{R}^{2}= \\
.043^{*}\end{array}$ \\
\hline
\end{tabular}

Note: ${ }^{*} p<.05,{ }^{* *} p<.01,{ }^{* * *} p<.001$ 
Table $9 b$. Predicted missingness of days in prison: Missingness at four survey time points regressed on days at the fifth survey time

\begin{tabular}{|c|c|c|c|c|c|c|}
\hline & \multicolumn{5}{|c|}{ Missing data $(0=$ missing; $1=$ present $)$} & \\
\hline & $\mathrm{T} 1$ & T2 & T3 & T4 & T5 & \\
\hline $\begin{array}{l}\text { Days at } \\
\text { ODYS at T1- } \\
\text { survey } \\
\text { (valid } \\
\text { response) }\end{array}$ & & $\begin{array}{l}b= \\
.50 .74, S E \\
=32.15, \beta \\
=.066\end{array}$ & $\begin{array}{l}b= \\
116.26, \\
S E= \\
.31 .26, \beta \\
=.154^{* * *}\end{array}$ & $\begin{array}{l}b= \\
134.62, \\
S E= \\
41.98, \beta= \\
.135 * *\end{array}$ & $\begin{array}{l}b= \\
.80 .86, \\
S E= \\
44.85, \beta \\
=.078\end{array}$ & $\begin{array}{l}\mathrm{R}^{2}= \\
.073 * * *\end{array}$ \\
\hline $\begin{array}{l}\text { Days at } \\
\text { ODYS at T2- } \\
\text { survey } \\
\text { (valid } \\
\text { response) }\end{array}$ & $\begin{array}{l}\mathrm{b}= \\
274.88, \\
S E= \\
25.19, \beta= \\
.372 * * *\end{array}$ & & $\begin{array}{l}b=53.07 \\
S E= \\
27.04, \beta= \\
.068\end{array}$ & $\begin{array}{l}b= \\
184.09, \\
S E= \\
38.52, \beta= \\
.189 * * *\end{array}$ & $\begin{array}{l}b= \\
144.08, \\
S E= \\
48.02, \beta \\
=.116^{* *}\end{array}$ & $\begin{array}{l}\mathrm{R}^{2}= \\
.209 * * *\end{array}$ \\
\hline $\begin{array}{l}\text { Days at } \\
\text { ODYS at T3- } \\
\text { survey } \\
\text { (valid } \\
\text { response) }\end{array}$ & $\begin{array}{l}b= \\
257.67, \\
S E= \\
24.39, \beta= \\
.348^{* * *}\end{array}$ & $\begin{array}{l}b= \\
103.45, \\
S E= \\
26.63, \beta= \\
.267 * * *\end{array}$ & & $\begin{array}{l}b= \\
212.65, \\
S E= \\
26.63, \beta= \\
.267^{* * *}\end{array}$ & $\begin{array}{l}b= \\
13.35, S E \\
=29.75, \\
\beta=.016\end{array}$ & $\begin{array}{l}\mathrm{R}^{2}= \\
.212 * * *\end{array}$ \\
\hline $\begin{array}{l}\text { Days at } \\
\text { ODYS at T4- } \\
\text { survey } \\
\text { (valid } \\
\text { response) }\end{array}$ & $\begin{array}{l}\mathrm{b}= \\
234.09, \\
S E= \\
.46 .08, \beta \\
=.322 * * *\end{array}$ & $\begin{array}{l}b= \\
150.13, \\
S E= \\
42.26, \beta= \\
.209 * * *\end{array}$ & $\begin{array}{l}b= \\
167.91, \\
S E= \\
61.43, \beta= \\
.171 * *\end{array}$ & & $\begin{array}{l}b= \\
118.78, \\
S E= \\
41.36, \beta \\
=.166^{* *}\end{array}$ & $\begin{array}{l}\mathrm{R}^{2}= \\
.165^{* * *}\end{array}$ \\
\hline $\begin{array}{l}\text { Days at } \\
\text { ODYS at T5- } \\
\text { survey } \\
\text { (valid } \\
\text { response) }\end{array}$ & $\begin{array}{l}\mathrm{b}= \\
177.91 \\
S E= \\
46.23, \beta= \\
.228 * * *\end{array}$ & $\begin{array}{l}b=194.9, \\
S E= \\
48.89, \beta= \\
.226 * * *\end{array}$ & $\begin{array}{l}b=64.03, \\
S E= \\
54.47, \beta= \\
.07\end{array}$ & $\begin{array}{l}b= \\
228.64, \\
S E= \\
42.23, \beta= \\
.304 * * *\end{array}$ & & $\begin{array}{l}\mathrm{R}^{2}= \\
.235^{* * *}\end{array}$ \\
\hline
\end{tabular}

Note: ${ }^{*} p<.05,{ }^{* *} p<.01,{ }^{* * *} p<.001$ 
Table 9c. Predicted missingness of ethnic pride: Missingness at four survey time points regressed on ethnic pride at the fifth survey time

\begin{tabular}{|c|c|c|c|c|c|c|}
\hline & \multicolumn{5}{|c|}{ Missing data $(0=$ missing; 1 = present $)$} & \\
\hline & T1 & $\mathrm{T} 2$ & T3 & T4 & T5 & \\
\hline $\begin{array}{l}\text { Ethnic pride } \\
\text { at T1-survey } \\
\text { (valid } \\
\text { response) }\end{array}$ & & $\begin{array}{l}b=.566 \\
S E= \\
1.034, \beta \\
=.023\end{array}$ & $\begin{array}{l}b=.975, \\
S E= \\
1.102, \beta \\
=.037\end{array}$ & $\begin{array}{l}b=-.816, \\
S E= \\
1.634, \beta \\
=-.022\end{array}$ & $\begin{array}{l}b= \\
1.989, \mathrm{SE} \\
=1.739, \\
\beta=1.144\end{array}$ & $\begin{array}{l}\mathrm{R}^{2}= \\
.004\end{array}$ \\
\hline $\begin{array}{l}\text { Ethnic pride } \\
\text { at T2-survey } \\
\text { (valid } \\
\text { response) }\end{array}$ & $\begin{array}{l}b= \\
1.45, S E \\
=.855, \\
\beta=.064\end{array}$ & & $\begin{array}{l}b=1.28, \\
S E=.87 \\
\beta=.056\end{array}$ & $\begin{array}{l}b=.831 \\
S E=1.43, \\
\beta=.026\end{array}$ & $\begin{array}{l}b=.698, \\
S E=1.6, \\
\beta=.019\end{array}$ & $\begin{array}{l}\mathrm{R}^{2}= \\
.01\end{array}$ \\
\hline $\begin{array}{l}\text { Ethnic pride } \\
\text { at T3-survey } \\
\text { (valid } \\
\text { response) }\end{array}$ & $\begin{array}{l}b=- \\
1.15, \mathrm{SE} \\
=1.33 \\
\beta=- \\
.036\end{array}$ & $\begin{array}{l}b=1.89 \\
S E=1.3 \\
\beta=.06\end{array}$ & & $\begin{array}{l}b=1.19 \\
S E=1.86 \\
\beta=.03\end{array}$ & $\begin{array}{l}b=-2.55 \\
S E=2.22 \\
\beta=-.055\end{array}$ & $\begin{array}{l}\mathrm{R}^{2}= \\
.005\end{array}$ \\
\hline $\begin{array}{l}\text { Ethnic pride } \\
\text { at T4-survey } \\
\text { (valid } \\
\text { response) }\end{array}$ & $\begin{array}{l}b= \\
2.86, S E \\
=1.93 \\
\beta=.092\end{array}$ & $\begin{array}{l}b=1.61 \\
S E= \\
2.072, \beta \\
=.053\end{array}$ & $\begin{array}{l}b=3.41, \\
S E= \\
2.01, \beta= \\
.114\end{array}$ & & $\begin{array}{l}b=.273 \\
S E=1.83 \\
\beta=.009\end{array}$ & $\begin{array}{l}\mathrm{R}^{2}= \\
.035\end{array}$ \\
\hline $\begin{array}{l}\text { Ethnic pride } \\
\text { at T5-survey } \\
\text { (valid } \\
\text { response) }\end{array}$ & $\begin{array}{l}b=- \\
.021, \mathrm{SE} \\
=2.17 \\
\beta=- \\
.001\end{array}$ & $\begin{array}{l}b=-2.36, \\
S E= \\
2.39, \beta= \\
-.076\end{array}$ & $\begin{array}{l}b=3.81, \\
S E= \\
2.51, \beta= \\
.122\end{array}$ & $\begin{array}{l}b=2.02, \\
S E=2.24, \\
\beta=.067\end{array}$ & & $\begin{array}{l}\mathrm{R}^{2}= \\
.021\end{array}$ \\
\hline
\end{tabular}

Note: ${ }^{*} \mathrm{p}<.05, * * \mathrm{p}<.01, * * * \mathrm{p}<.001$ 
Table 9d. Predicted missingness of ethnic identity: Missingness at four survey time points related to ethnic identity at the fifth time point

\begin{tabular}{|c|c|c|c|c|c|}
\hline & \multicolumn{5}{|c|}{ Missing data $(0=$ missing $; 1=$ present $)$} \\
\hline & T1 & T2 & T3 & T4 & T5 \\
\hline $\begin{array}{l}\text { Ethnic } \\
\text { identity at } \\
\text { T1-survey } \\
\text { (valid } \\
\text { response) }\end{array}$ & & $\begin{array}{l}\chi 2(5)= \\
9.51, p= \\
.09 ; \\
\text { Cramer's V } \\
=.115\end{array}$ & $\begin{array}{l}\chi 2(5)= \\
12.09, \mathrm{p}= \\
.034 ; \\
\text { Cramer's V } \\
=.129\end{array}$ & $\begin{array}{l}\chi 2(5)= \\
2.06, p= \\
.841 ; \\
\text { Cramer's V } \\
=.053\end{array}$ & $\begin{array}{l}\chi 2(5)= \\
4.09, p= \\
.536 ; \\
\text { Cramer's V } \\
=.075\end{array}$ \\
\hline $\begin{array}{l}\text { Ethnic } \\
\text { identity at } \\
\text { T2-survey } \\
\text { (valid } \\
\text { response) }\end{array}$ & $\begin{array}{l}\chi 2(6)= \\
7.50, p= \\
.277 ; \\
\text { Cramer's V } \\
=.102\end{array}$ & & $\begin{array}{l}\chi 2(6)= \\
5.19, p= \\
.519 ; \\
\text { Cramer's V } \\
=.085\end{array}$ & $\begin{array}{l}\chi 2(6)= \\
3.06, p= \\
.801 ; \\
\text { Cramer's V } \\
=.065\end{array}$ & $\begin{array}{l}\chi 2(6)= \\
7.25, p= \\
.298 ; \\
\text { Cramer's V } \\
=.101\end{array}$ \\
\hline $\begin{array}{l}\text { Ethnic } \\
\text { identity at } \\
\text { T3-survey } \\
\text { (valid } \\
\text { response) }\end{array}$ & $\begin{array}{l}\chi 2(6)= \\
12.81, p= \\
.046 ; \\
\text { Cramer's V } \\
=.137\end{array}$ & $\begin{array}{l}\chi 2(6)= \\
6.56, p= \\
.363 ; \\
\text { Cramer's V } \\
=.098\end{array}$ & & $\begin{array}{l}\chi 2(6)= \\
8.37, p= \\
.212 ; \\
\text { Cramer's V } \\
=.111\end{array}$ & $\begin{array}{l}\chi 2(6)= \\
6.74, p= \\
.346 ; \\
\text { Cramer's V } \\
=.1\end{array}$ \\
\hline $\begin{array}{l}\text { Ethnic } \\
\text { identity at } \\
\text { T4-survey } \\
\text { (valid } \\
\text { response) }\end{array}$ & $\begin{array}{l}\chi 2(6)= \\
5.34, p= \\
.501 ; \\
\text { Cramer's V } \\
=.141\end{array}$ & $\begin{array}{l}\chi 2(6)= \\
5.70, p= \\
.458 ; \\
\text { Cramer's V } \\
=.146\end{array}$ & $\begin{array}{l}\chi 2(6)= \\
6.11, p= \\
.411 ; \\
\text { Cramer's V } \\
=.151\end{array}$ & & $\begin{array}{l}\chi 2(6)= \\
11.76, p= \\
.068 ; \\
\text { Cramer's V } \\
=.209\end{array}$ \\
\hline $\begin{array}{l}\text { Ethnic } \\
\text { identity at } \\
\text { T5-survey } \\
\text { (valid } \\
\text { response) }\end{array}$ & $\begin{array}{l}\chi 2(6)= \\
6.46, p= \\
.373 ; \\
\text { Cramer's V } \\
=.170\end{array}$ & $\begin{array}{l}\chi 2(6)= \\
7.73, p= \\
.259 ; \\
\text { Cramer's V } \\
=.186\end{array}$ & $\begin{array}{l}\chi 2(6)= \\
4.83, p= \\
.566 ; \\
\text { Cramer's V } \\
=.147\end{array}$ & $\begin{array}{l}\chi 2(6)= \\
1.02, p= \\
.985 ; \\
\text { Cramer's V } \\
=.068\end{array}$ & \\
\hline
\end{tabular}


Table 10. Colinearity diagnostics of level-2 predictor variables

\begin{tabular}{|l|c|c|c|c|c|c|c|}
\hline & Age & $\begin{array}{c}\text { Days in } \\
\text { Prison }\end{array}$ & $\begin{array}{c}\text { Ethnic } \\
\text { Pride }\end{array}$ & White & Latino & Other & $\begin{array}{c}\text { The } \\
\text { Council }\end{array}$ \\
\hline Age & & & & & & & \\
\hline $\begin{array}{l}\text { Days in } \\
\text { Prison }\end{array}$ & $.324 * *$ & & & & & & \\
\hline $\begin{array}{l}\text { Ethnic } \\
\text { Pride }\end{array}$ & $.084 * *$ & -.014 & & & & & \\
\hline White & $-.058 * *$ & $-.088^{* *}$ & .023 & & & & \\
\hline Latino & .007 & .015 & $.038^{*}$ & -- & & & \\
\hline Other & .007 & .000 & .001 & -- & -- & & \\
\hline $\begin{array}{l}\text { The } \\
\text { Council }\end{array}$ & .164 & $.381 * *$ & .000 & $-.147 * *$ & .016 & -.017 & \\
\hline
\end{tabular}

Notes: Correlation coefficients: Pearson (two continuous variables), Point-Biserial (one continuous, one dichotomous), Phi (two dichotomous).

$* * p<.001, * p<.05$ 
Table 11a. Model Trimming: Complete Model (Model 1)

Complete Model (Model 1)

-2 Log Likelihood

1153.98

Number of parameters

Intercept, $\gamma_{0 i}$

Occasion2, $\gamma_{10}$

Occasion $3, \gamma_{20}$

The Council, $\gamma_{01}$

Age, $\gamma_{02}$

Days in prison, $\gamma_{03}$

White, $\gamma_{04}$

Latino, $\gamma_{05}$

Other, $\gamma_{06}$

Ethnic Pride, $\gamma_{07}$

Occasion $2 *$ Council, $\gamma_{11}$

Occasion $3 *$ Council, $\gamma_{21}$

Occasion $2^{*}$ Age, $\gamma_{31}$

Occasion $3 *$ Age, $\gamma_{41}$

Occasion2*Days, $\gamma_{51}$

Occasion3*Days, $\gamma_{61}$

Occasion $2 *$ White, $\gamma_{71}$

Occasion $3 *$ White, $\gamma_{81}$

Occasion $2 *$ Latino, $\gamma_{91}$

Occasion3* Latino, $\gamma_{10.1}$

Pride*White, $\gamma_{11.1}$

Pride*Latino, $\gamma_{12.1}$

Pride*Occasion2, $\gamma_{13.1}$

Pride*Occasion3, $\gamma_{14.1}$

The Council*Age, $\gamma_{15.1}$

The Council*Days, $\gamma_{16.1}$

Pride*Occasion $2 *$ White, $\gamma_{17.1}$

Pride*Occasion $3 *$ White, $\gamma_{18.1}$

Pride*Occasion $2 *$ Latino, $\gamma_{19.1}$

Pride*Occasion $3 *$ Latino, $\gamma_{20.1}$

Occ. $2 *$ The Council*Age, $\gamma_{21.1}$

Occ. $3^{*}$ The Council*Age, $\gamma_{22.1}$

Occ. $2 *$ The Council*Days, $\gamma_{23.1}$

Occ. $3 *$ The Council*Days, $\gamma_{24.1}$

Coefficient

2.26

$-.010$

$-.017$

.069

$-.054$

$-6.29 \mathrm{E}^{-6}$

$-.301$

$-.339$

$-.157$

$-.032$

.027

.108

$-.002$

$-.001$

$5.97 \mathrm{E}^{-5}$

$2.92 \mathrm{E}^{-5}$

.052

.054

.389

.089

$-.008$

.235

$-.033$

$-.027$

.029

$4.97 \mathrm{E}^{-5}$

$-.078$

$-.041$

$-.406$

$-.133$

$-.003$

.038

$-.0002$

$-.0002$
36

SE

0.024

.025

.026

.041

.012

$6.66 \mathrm{E}^{-5}$

.039

.178

.041

.017

.048

.055

.002

.002

$7.26 \mathrm{E}^{-5}$

$7.03 \mathrm{E}^{-5}$

.042

.044

.176

.220

.033

.206

.019

.019

.030

.0001

.041

.041

.203

.248

.029

.030

.0001

.0001 p-value

.000

.698

.513

.092

.000

.925

.000

.058

.000

.059

.573

.048

.352

.412

.411

.678

.217

.219

.027

.684

.814

.252

.079

.157

.329

.642

.058

.331

.045

.592

.922

.214

.183

.098

Residual, $e_{t i}$

Random Effects

Intercept, $u_{0 i}$ 
Table 11b. Model Trimming: Model 2

-2 Log Likelihood

1156.71

Number of parameters

Intercept, $\gamma_{0 i}$

Occasion2, $\gamma_{10}$

Occasion3, $\gamma_{20}$

The Council, $\gamma_{01}$

Age, $\gamma_{02}$

Days in prison, $\gamma_{03}$

White, $\gamma_{04}$

Latino, $\gamma_{05}$

Other, $\gamma_{06}$

Ethnic Pride, $\gamma_{07}$

Occasion $2 *$ Council, $\gamma_{11}$

Occasion $3 *$ Council, $\gamma_{21}$

Occasion $2 *$ Age, $\gamma_{31}$

Occasion $3 *$ Age, $\gamma_{41}$

Occasion $2 *$ Days, $\gamma_{51}$

Occasion $3 *$ Days, $\gamma_{61}$

Occasion $2 *$ White, $\gamma_{71}$

Occasion $3 *$ White, $\gamma_{81}$

Occasion $2 *$ Latino, $\gamma_{91}$

Occasion3*Latino, $\gamma_{10.1}$

Pride*White, $\gamma_{11.1}$

Pride*Latino, $\gamma_{12.1}$

Pride*Occasion2, $\gamma_{13.1}$

Pride*Occasion3, $\gamma_{14.1}$

The Council*Age, $\gamma_{15.1}$

The Council*Days, $\gamma_{16.1}$

Pride*Occasion $2 *$ White, $\gamma_{17.1}$

Pride*Occasion $3 *$ White, $\gamma_{18.1}$

Pride*Occasion $2 *$ Latino, $\gamma_{19.1}$

Pride*Occasion $3 *$ Latino, $\gamma_{20.1}$

Occ. $2 *$ The Council*Age, $\gamma_{21.1}$

Occ. $3 *$ The Council*Age, $\gamma_{22.1}$

Occ. $2 *$ The Council*Days, $\gamma_{23.1}$

Occ. $3 *$ The Council*Days, $\gamma_{24.1}$

Random Effects

Residual, $e_{t i}$

\section{Coefficient}

2.25

$-.007$

$-.015$

.088

$-.054$

$2.49 \mathrm{E}^{-5}$

$-.300$

$-.334$

$-.156$

$-.032$

.011

.077

$-.002$

$-.002$

$2.71 \mathrm{E}^{-5}$

$-3.82 \mathrm{E}^{-5}$

.052

.053

.383

.076

$-.008$

.246

$-.033$

$-.027$

.038

$-4.12 \mathrm{E}^{-5}$

$-.078$

$-.040$

$-.415$

$-.146$

$-.012$

.019

$-5.74 \mathrm{E}^{-5}$

---
SE

.024

.025

.026

.039

.012

$6.38 \mathrm{E}^{-5}$

.039

.178

.041

.017

.047

.051

.002

.002

$6.99 \mathrm{E}^{-5}$

$5.74 \mathrm{E}^{-5}$

.042

.044

.176

.220

.033

.205

.019

.019

.029

9. $18 \mathrm{E}^{-5}$

.041

.041

.203

.248

.029

.028

$9.78 \mathrm{E}^{-5}$
35

p-value

.000

.762

.548

.026

.000

.696

.000

.061

.000

.059

.820

.134

.349

.366

.699

.507

.216

.229

.030

.731

.814

.231

.080

.147

.194

.653

.058

.332

.041

.555

.667

.449

.557

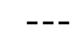

Intercept, $u_{0 i}$ 
Table 11c. Model Trimming: Model 3

-2 Log Likelihood

1157.06

Number of parameters

Intercept, $\gamma_{0 i}$

Occasion2, $\gamma_{10}$

Occasion $3, \gamma_{20}$

The Council, $\gamma_{01}$

Age, $\gamma_{02}$

Days in prison, $\gamma_{03}$

White, $\gamma_{04}$

Latino, $\gamma_{05}$

Other, $\gamma_{06}$

Ethnic Pride, $\gamma_{07}$

Occasion $2 *$ Council, $\gamma_{11}$

Occasion $3 *$ Council, $\gamma_{21}$

Occasion $2 *$ Age, $\gamma_{31}$

Occasion $3^{*}$ Age, $\gamma_{41}$

Occasion $2 *$ Days, $\gamma_{51}$

Occasion $3 *$ Days, $\gamma_{61}$

Occasion $2 *$ White, $\gamma_{71}$

Occasion $3 *$ White, $\gamma_{81}$

Occasion2*Latino, $\gamma_{91}$

Occasion3* Latino, $\gamma_{10.1}$

Pride*White, $\gamma_{11.1}$

Pride*Latino, $\gamma_{12.1}$

Pride*Occasion2, $\gamma_{13.1}$

Pride*Occasion3, $\gamma_{14.1}$

The Council*Age, $\gamma_{15.1}$

The Council*Days, $\gamma_{16.1}$

Pride*Occasion $2 *$ White, $\gamma_{17.1}$

Pride*Occasion $3 *$ White, $\gamma_{18.1}$

Pride*Occasion $2 *$ Latino, $\gamma_{19.1}$

Pride*Occasion $3 *$ Latino, $\gamma_{20.1}$

Occ. $2 *$ The Council*Age, $\gamma_{21.1}$

Occ. $3 *$ The Council*Age, $\gamma_{22.1}$

Occ. $2 *$ The Council*Days, $\gamma_{23.1}$

Occ. $3 *$ The Council*Days, $\gamma_{24.1}$

Residual, $e_{t i}$

Intercept, $u_{0 i}$

\section{Random Effects}

\section{Coefficient}

2.25

$-.008$

$-.015$

.091

$-.054$

3. $19 \mathrm{E}^{-5}$

$-.300$

$-.334$

$-.156$

$-.032$

.004

.076

$-.002$

$-.001$

$1.97 \mathrm{E}^{-6}$

$-3.90 E^{-5}$

.051

.053

.379

.076

$-.008$

.248

$-.033$

$-.027$

.040

$-6.02 E^{-5}$

$-.078$

$-.040$

$-.419$

$-.147$

$-.017$

.019

---
SE

.024

.025

.026

.039

.012

$6.27 \mathrm{E}^{-5}$

.039

.178

.041

.017

.046

.051

.002

.002

$5.53 \mathrm{E}^{-5}$

$5.74 \mathrm{E}^{-5}$

.042

.044

.176

.220

.033

.206

.019

.019

.029

$8.59 \mathrm{E}^{-5}$

.041

.041

.203

.248

.027

.028

---

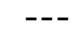

34

p-value

.000

.761

.565

.020

.000

.661

.000

.061

.000

.059

.932

.140

.342

.377

.972

.497

.222

.225

.032

.730

.815

.228

.082

.148

.166

.483

.058

.326

.039

.553

.521

.510

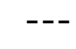


Table 11d. Model Trimming: Model 4

$$
\text { -2 Log Likelihood }
$$

1157.52

Number of parameters

Intercept, $\gamma_{o i}$

Occasion2, $\gamma_{10}$

Occasion $3, \gamma_{20}$

The Council, $\gamma_{01}$

Age, $\gamma_{02}$

Days in prison, $\gamma_{03}$

White, $\gamma_{04}$

Latino, $\gamma_{05}$

Other, $\gamma_{06}$

Ethnic Pride, $\gamma_{07}$

Occasion $2 *$ Council, $\gamma_{11}$

Occasion $3 *$ Council, $\gamma_{21}$

Occasion2*Age, $\gamma_{31}$

Occasion $3 *$ Age, $\gamma_{41}$

Occasion $2 *$ Days, $\gamma_{51}$

Occasion $3 *$ Days, $\gamma_{61}$

Occasion $2 *$ White, $\gamma_{71}$

Occasion $3 *$ White, $\gamma_{81}$

Occasion2* Latino, $\gamma_{91}$

Occasion3*Latino, $\gamma_{10.1}$

Pride*White, $\gamma_{11.1}$

Pride*Latino, $\gamma_{12.1}$

Pride*Occasion2, $\gamma_{13.1}$

Pride*Occasion $3, \gamma_{14.1}$

The Council*Age, $\gamma_{15.1}$

The Council*Days, $\gamma_{16.1}$

Pride*Occasion $2 *$ White, $\gamma_{17.1}$

Pride*Occasion $3 *$ White, $\gamma_{18.1}$

Pride* Occasion $2 *$ Latino, $\gamma_{19.1}$

Pride*Occasion3*Latino, $\gamma_{20.1}$

Occ. ${ }^{*}$ The Council*Age, $\gamma_{21.1}$

Occ. ${ }^{*}$ The Council*Age, $\gamma_{22.1}$

Occ. $2 *$ The Council*Days, $\gamma_{23.1}$

Occ. $3 *$ The Council $*$ Days, $\gamma_{24.1}$

Random Effects

Residual, $e_{t i}$

Intercept, $u_{0 i}$

\section{Coefficient}

2.25

$-.008$

$-.015$

.094

$-.054$

$1.41 \mathrm{E}^{-5}$

$-.300$

$-.333$

$-.156$

$-.032$

.001

.068

$-.002$

$-.002$

$2.03 \mathrm{E}^{-5}$

---

.051

.054

.380

.079

$-.008$

.249

$-.033$

$-.027$

.043

$-6.04 \mathrm{E}^{-5}$

$-.077$

$-.040$

$-.422$

$-.154$

$-.020$

.013

---
SE

.024

.025

.026

.039

.012

$5.69 \mathrm{E}^{-5}$

.039

.178

.041

.017

.046

.050

.002

.002

$4.85 \mathrm{E}^{-5}$

---

.042

.044

.176

.220

.033

.205

.019

.019

.029

$8.59 \mathrm{E}^{-5}$

.041

.041

.203

.258

.027

.027

---

.060

.101

.003

.007
33

$p$-value

.000

.733

.554

.015

.000

.805

.000

.062

.000

.057

.990

.176

.341

.364

.676

.227

.220

.032

.721

.802

.226

.084

.151

.134

.482

.061

.332

.038

.535

.447

.627
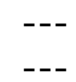

.000

.000 
Table 11e. Model Trimming: Model 5

-2 Log Likelihood

1157.69

Number of parameters

Intercept, $\gamma_{0 i}$

Occasion2, $\gamma_{10}$

Occasion3, $\gamma_{20}$

The Council, $\gamma_{01}$

Age, $\gamma_{02}$

Days in prison, $\gamma_{03}$

White, $\gamma_{04}$

Latino, $\gamma_{05}$

Other, $\gamma_{06}$

Ethnic Pride, $\gamma_{07}$

Occasion $2 *$ Council, $\gamma_{11}$

Occasion $3 *$ Council, $\gamma_{21}$

Occasion ${ }^{*}$ Age, $\gamma_{31}$

Occasion $3^{*}$ Age, $\gamma_{41}$

Occasion $2 *$ Days, $\gamma_{51}$

Occasion $3 *$ Days, $\gamma_{61}$

Occasion $2 *$ White, $\gamma_{71}$

Occasion $3 *$ White, $\gamma_{81}$

Occasion $2 *$ Latino, $\gamma_{91}$

Occasion3* Latino, $\gamma_{10.1}$

Pride*White, $\gamma_{11.1}$

Pride*Latino, $\gamma_{12.1}$

Pride*Occasion2, $\gamma_{13.1}$

Pride*Occasion3, $\gamma_{14.1}$

The Council*Age, $\gamma_{15.1}$

The Council*Days, $\gamma_{16.1}$

Pride*Occasion $2 *$ White, $\gamma_{17.1}$

Pride*Occasion $3 *$ White, $\gamma_{18.1}$

Pride*Occasion $2 *$ Latino, $\gamma_{19.1}$

Pride*Occasion $3 *$ Latino, $\gamma_{20.1}$

Occ. $2 *$ The Council*Age, $\gamma_{21.1}$

Occ. $3 *$ The Council*Age, $\gamma_{22.1}$

Occ. $2 *$ The Council*Days, $\gamma_{23.1}$

Occ. $3 *$ The Council*Days, $\gamma_{24.1}$

Random Effects

Residual, $e_{t i}$

Intercept, $u_{0 i}$

\section{Coefficient}

2.25

$-.009$

$-.015$

.093

$-.054$

$1.96 \mathrm{E}^{-5}$

$-.300$

$-.333$

$-.156$

$-.032$

.005

.067

$-.002$

$-.002$

---

.050

.055

.380

.079

$-.008$

.249

$-.033$

$-.027$

.042

$-5.81 \mathrm{E}^{-5}$

$-.078$

$-.040$

$-.420$

$-.155$

$-.017$

.014

---
SE

.024

.025

.026

.039

.012

$5.54 \mathrm{E}^{-5}$

.039

.178

.041

.017

.044

.050

.002

.002

---

042

.042

.176

.220

.033

.205

.019

.019

.029

$8.57 \mathrm{E}^{-5}$

.041

.041

.203

.248

.026

.027

--.

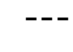

32

p-value

.000

.731

.567

.016

.000

.724

.000

.062

.000

.057

.907

.117

.345

.356

---

.232

.215

.032

.718

.812

.226

.084

.150

.144

.498

.057

.327

.039

.533

.504

.617

---

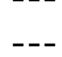


Table 11f. Model Trimming: Model 6

-2 Log Likelihood

1158.15

Number of parameters

Intercept, $\gamma_{o i}$

Occasion2, $\gamma_{10}$

Occasion $3, \gamma_{20}$

The Council, $\gamma_{01}$

Age, $\gamma_{02}$

Days in prison, $\gamma_{03}$

White, $\gamma_{04}$

Latino, $\gamma_{05}$

Other, $\gamma_{06}$

Ethnic Pride, $\gamma_{07}$

Occasion $2 *$ Council, $\gamma_{11}$

Occasion $3 *$ Council, $\gamma_{21}$

Occasion2*Age, $\gamma_{31}$

Occasion3*Age, $\gamma_{41}$

Occasion $2 *$ Days, $\gamma_{51}$

Occasion $3 *$ Days, $\gamma_{61}$

Occasion $2 *$ White, $\gamma_{71}$

Occasion $3 *$ White, $\gamma_{81}$

Occasion $2 *$ Latino, $\gamma_{91}$

Occasion3*Latino, $\gamma_{10.1}$

Pride*White, $\gamma_{11.1}$

Pride*Latino, $\gamma_{12.1}$

Pride*Occasion2, $\gamma_{13.1}$

Pride*Occasion $3, \gamma_{14.1}$

The Council*Age, $\gamma_{15,1}$

The Council*Days, $\gamma_{16.1}$

Pride*Occasion $2 *$ White, $\gamma_{17.1}$

Pride*Occasion $3 *$ White, $\gamma_{18.1}$

Pride*Occasion $2 *$ Latino, $\gamma_{19.1}$

Pride*Occasion $3 *$ Latino, $\gamma_{20.1}$

Occ. $2 *$ The Council*Age, $\gamma_{2 l .1}$

Occ. ${ }^{*}$ The Council*Age, $\gamma_{22.1}$

Occ. $2 *$ The Council*Days, $\gamma_{23.1}$

Occ. ${ }^{*}$ The Council*Days, $\gamma_{24.1}$

Random Effects

Residual, $e_{t i}$

Intercept, $u_{0 i}$

\section{Coefficient}

2.25

$-.009$

$-.016$

.087

$-.053$

$-4.56 \mathrm{E}^{-6}$

$-.300$

$-.332$

$-.154$

$-.032$

.006

.070

$-.002$

$-.002$

---

.050

.054

.378

.077

$-.008$

.244

$-.033$

$-.027$

.036

$-.078$

$-.040$

$-.419$

$-.153$

$-.017$

.014

$--$
SE

.024

.025

.026

.038

.012

$4.25 \mathrm{E}^{-5}$

.039

.178

.041

.017

.044

.050

.002

.002

---

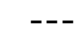

.042

.044

.176

.220

.033

.205

.019

.019

.027

041

.041

.203

.248

.026

.027

--.

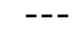

31

p-value

.000

.714

.544

.021

.000

.914

.000

.063

.000

.057

.889

.156

.331

.325

---

.235

.219

.032

.726

.821

.235

.084

.148

.188

.058

.334

.039

.537

.511

.605

--- 
Table 11g. Model Trimming: Final Model (Model 7)

-2 Log Likelihood

Number of parameters

Intercept, $\gamma_{0 i}$

Occasion2, $\gamma_{10}$

Occasion3, $\gamma_{20}$

The Council, $\gamma_{01}$

Age, $\gamma_{02}$

Days in prison, $\gamma_{03}$

White, $\gamma_{04}$

Latino, $\gamma_{05}$

Other, $\gamma_{06}$

Ethnic Pride, $\gamma_{07}$

Occasion $2 *$ Council, $\gamma_{11}$

Occasion $3 *$ Council, $\gamma_{21}$

Occasion ${ }^{*}$ Age, $\gamma_{31}$

Occasion $3^{*}$ Age, $\gamma_{41}$

Occasion $2 *$ Days, $\gamma_{51}$

Occasion $3 *$ Days, $\gamma_{61}$

Occasion $2 *$ White, $\gamma_{71}$

Occasion $3 *$ White, $\gamma_{81}$

Occasion $2 *$ Latino, $\gamma_{91}$

Occasion3*Latino, $\gamma_{10.1}$

Pride*White, $\gamma_{11.1}$

Pride*Latino, $\gamma_{12.1}$

Pride*Occasion2, $\gamma_{13.1}$

Pride*Occasion3, $\gamma_{14.1}$

The Council*Age, $\gamma_{15.1}$

The Council*Days, $\gamma_{16.1}$

Pride*Occasion $2 *$ White, $\gamma_{17.1}$

Pride*Occasion $3 *$ White, $\gamma_{18.1}$

Pride*Occasion $2 *$ Latino, $\gamma_{19.1}$

Pride*Occasion $3 *$ Latino, $\gamma_{20.1}$

Occ. $2 *$ The Council*Age, $\gamma_{21.1}$

Occ. $3 *$ The Council*Age, $\gamma_{22.1}$

Occ. $2 *$ The Council*Days, $\gamma_{23.1}$

Occ. $3 *$ The Council*Days, $\gamma_{24.1}$

Random Effects

Residual, $e_{t i}$

Intercept, $u_{0 i}$

\section{Coefficient}

2.25

$-.014$

$-.024$

.196

$-.041$

$-.283$

$-.120$

$-.120$

$-.032$

.026

.070

$-.013$

$-.019$

---

.088

.090

.128

$-.008$

$-.017$

.041

$-.024$

$-.019$

.044

$-.040$

$-.022$

$-.108$

.004

$-.013$

.019

---

.061

.095 $1529.20^{\mathrm{a}}$

30

SE

.021

.023

.024

$p$-value

.000

.539

.326

.031

.010

.002

.000

.034

.106

.035

.014

.037

.042

.019

.020

000

.258

.001

.021

.484

.094

.486

.348

---

.039

.041

.131

.140

.029

.094

.016

.017

.019

.025

.028

.331

.957

.554

.661

.141

.265

.025

.038

.037

.125

.130

.019

.021

---

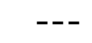

.294

.556

.386

.975

.490

.350

---

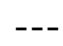


Table 12. Model parameter comparisons between samples

\begin{tabular}{|c|c|c|c|c|c|}
\hline & & $\mathrm{N}$ & Means & Statistical test & $P$ value \\
\hline \multirow[t]{2}{*}{ Age } & Included & 873 & 16.68 & \multirow[b]{2}{*}{$t(1241)=6.00$} & \multirow[b]{2}{*}{$<.001$} \\
\hline & $\begin{array}{c}\text { Not } \\
\text { included }\end{array}$ & 370 & 17.19 & & \\
\hline \multirow[t]{2}{*}{ The Council } & Included & 876 & & \multirow[b]{2}{*}{$X^{2}(1)=68.64$} & \multirow[b]{2}{*}{$<.001$} \\
\hline & $\begin{array}{c}\text { Not } \\
\text { included }\end{array}$ & 372 & & & \\
\hline \multirow[t]{2}{*}{ Ethnic Pride } & Included & 869 & 3.044 & \multirow[b]{2}{*}{$t(1227)=-1.85$} & \multirow[b]{2}{*}{.065} \\
\hline & $\begin{array}{c}\text { Not } \\
\text { included }\end{array}$ & 360 & 2.90 & & \\
\hline \multirow[t]{2}{*}{ AMIRS T1 } & Included & 477 & 2.18 & \multirow{2}{*}{$t(642)=1.06$} & \multirow[b]{2}{*}{.292} \\
\hline & $\begin{array}{c}\text { Not } \\
\text { included }\end{array}$ & 167 & 2.22 & & \\
\hline \multirow[t]{2}{*}{ AMIRS T2 } & Included & 507 & 2.19 & \multirow[b]{2}{*}{$t(667)=3.51$} & \multirow[b]{2}{*}{$<.001$} \\
\hline & $\begin{array}{c}\text { Not } \\
\text { included }\end{array}$ & 162 & 2.32 & & \\
\hline \multirow[t]{2}{*}{ AMIRS T3 } & Included & 510 & 2.56 & \multirow[b]{2}{*}{$t(627)=1.61$} & \multirow[b]{2}{*}{.108} \\
\hline & $\begin{array}{c}\text { Not } \\
\text { included }\end{array}$ & 119 & 2.29 & & \\
\hline \multirow[t]{2}{*}{ Race/ethnicity } & Included & 875 & & \multirow[b]{2}{*}{$X^{2}(6)=15.36$} & \multirow[b]{2}{*}{.018} \\
\hline & $\begin{array}{c}\text { Not } \\
\text { included }\end{array}$ & 370 & & & \\
\hline
\end{tabular}


Table 13. Summary of Hypothesis Testing

\begin{tabular}{|c|c|c|}
\hline Hypotheses & & Study Findings \\
\hline \multirow{2}{*}{$\begin{array}{l}\text { H1. Program } \\
\text { Effect }\end{array}$} & 1a. Program Effect & Not supported \\
\hline & 1b. Dosage effect & Supported \\
\hline \multirow{2}{*}{ H2. Age Effect } & 2a. Age predict initial levels & Supported \\
\hline & 2b. Age predict change over time & Not supported \\
\hline \multirow{2}{*}{$\begin{array}{l}\mathrm{H} 3 . \\
\text { Race/Ethnicity }\end{array}$} & 3a. Race/Ethnicity predict initial levels & $\begin{array}{l}\text { Partially } \\
\text { supported }\end{array}$ \\
\hline & $\begin{array}{l}\text { 3b. Race/Ethnicity predict change over } \\
\text { time }\end{array}$ & $\begin{array}{l}\text { Partially } \\
\text { supported }\end{array}$ \\
\hline \multirow{2}{*}{ H4. Ethnic Pride } & 4a. Ethnic pride predict initial levels & $\begin{array}{l}\text { Partially } \\
\text { supported }\end{array}$ \\
\hline & $\begin{array}{l}\text { 4b. Ethnic pride predict change over } \\
\text { time }\end{array}$ & Not supported \\
\hline \multirow[b]{2}{*}{ H5. Prison Effect } & 5a. Days in prison predict initial levels & Not supported \\
\hline & $\begin{array}{l}\text { 5b. Days in prison predict change over } \\
\text { time }\end{array}$ & Not supported \\
\hline
\end{tabular}


Table 14. Qualitative Analysis: Phase 1: Data extraction process

\begin{tabular}{|l|l|l|l|l|}
\hline & \multicolumn{1}{|c|}{ Data set } & \multicolumn{1}{|c|}{ Data item } & Data Extract1 & \multicolumn{1}{|c|}{$\begin{array}{c}\text { Final Data } \\
\text { Extract }\end{array}$} \\
\hline S8 & $996(100 \%)$ & $408(41 \%)$ & $342(34 \%)$ & $287(29 \%)$ \\
\hline S9 & $996(100 \%)$ & $392(39 \%)$ & $392(39 \%)$ & $392(39 \%)$ \\
\hline S10 & $996(100 \%)$ & $372(37 \%)$ & $225(23 \%)$ & $111(11 \%)$ \\
\hline S11 & $996(100 \%)$ & $385(39 \%)$ & $191(19 \%)$ & $89(9 \%)$ \\
\hline Total & 3,984 & $1,557(39 \%)$ & $1,150(29 \%)$ & $897(23 \%)$ \\
\hline
\end{tabular}


Table 15. Qualitative Analysis: Phase 3: Searching for themes

\begin{tabular}{|c|c|c|c|c|}
\hline $\begin{array}{l}\text { Emotional } \\
\text { stoicism }\end{array}$ & $\begin{array}{l}\text { Heterosexual } \\
\text { dominance }\end{array}$ & $\begin{array}{l}\text { Physical } \\
\text { toughness }\end{array}$ & $\begin{array}{l}\text { Competitive } \\
\& \text { ambition }\end{array}$ & Other \\
\hline $\begin{array}{l}\text { Emotional } \\
\text { expression }\end{array}$ & $\begin{array}{l}\text { Masculine } \\
\text { dominance }\end{array}$ & $\begin{array}{l}\text { Avoid fights and } \\
\text { conflict }\end{array}$ & $\begin{array}{l}\text { Relational: } \\
\text { commonality } \\
\text { with others }\end{array}$ & $\begin{array}{c}\text { General } \\
\text { group: Yes, } \\
\text { No, ... }\end{array}$ \\
\hline $\begin{array}{l}\text { Emotional in- } \\
\text { expression }\end{array}$ & $\begin{array}{c}\text { Man up } \\
\text { (expectation to } \\
\text { act like a man) }\end{array}$ & $\begin{array}{l}\text { Expectation to be } \\
\text { tough/strong }\end{array}$ & $\begin{array}{l}\text { Leadership/M } \\
\text { entoring }\end{array}$ & $\begin{array}{c}\text { Awareness of } \\
\text { consequences } \\
\text { of actions }\end{array}$ \\
\hline $\begin{array}{c}\text { New } \\
\text { perspective }\end{array}$ & $\begin{array}{l}\text { Positive man } \\
\text { (opposing male } \\
\text { expectation) }\end{array}$ & $\begin{array}{c}\text { Awareness that } \\
\text { men do not have } \\
\text { to act tough to be } \\
\text { cool }\end{array}$ & $\begin{array}{l}\text { Status - } \\
\text { general }\end{array}$ & $\begin{array}{l}\text { Personal } \\
\text { growth }\end{array}$ \\
\hline--- & --- & --- & $\begin{array}{l}\text { Helping } \\
\text { behaviors }\end{array}$ & $\begin{array}{c}\text { Problem } \\
\text { solving skills }\end{array}$ \\
\hline--- & --- & --- & Self-efficacy & Family roles \\
\hline
\end{tabular}


Table 16. Qualitative Analysis: Phase 4: Final Themes and Sub-themes

\begin{tabular}{|c|c|c|c|c|c|c|c|}
\hline $\begin{array}{c}\text { Emotional } \\
\text { stoicism }\end{array}$ & $\begin{array}{c}\text { Heterosexual } \\
\text { dominance }\end{array}$ & $\begin{array}{c}\text { Physical } \\
\text { toughness }\end{array}$ & $\begin{array}{l}\text { Competitive } \\
\text { \& ambition }\end{array}$ & $\begin{array}{c}\text { The Council - } \\
\text { Group } \\
\text { Specific }\end{array}$ & "Man Up" & $\begin{array}{c}\text { New } \\
\text { perspective }\end{array}$ & Relational \\
\hline $\begin{array}{l}\text { Emotional } \\
\text { expression }\end{array}$ & $\begin{array}{l}\text { Masculine } \\
\text { dominance }\end{array}$ & $\begin{array}{l}\text { Avoid } \\
\text { fights and } \\
\text { conflict }\end{array}$ & Self-efficacy & $\begin{array}{c}\text { General group: } \\
\text { Yes, No, ... }\end{array}$ & $\begin{array}{c}\text { Man up } \\
\text { (expectation } \\
\text { to be a man) }\end{array}$ & $\begin{array}{c}\text { New } \\
\text { perspective }\end{array}$ & $\begin{array}{c}\text { Relational: } \\
\text { commonalit } \\
\text { y with } \\
\text { others }\end{array}$ \\
\hline \multirow[t]{2}{*}{$\begin{array}{l}\text { Emotional } \\
\text { stoicism }\end{array}$} & & $\begin{array}{l}\text { Expectation } \\
\text { to be } \\
\text { tough/stron } \\
\mathrm{g}\end{array}$ & $\begin{array}{l}\text { Leadership/ } \\
\text { Mentoring }\end{array}$ & & $\begin{array}{l}\text { Positive man } \\
\quad(\text { opposing } \\
\text { expectation })^{1}\end{array}$ & $\begin{array}{l}\text { Personal } \\
\text { growth }\end{array}$ & Family roles \\
\hline & & & Status & & $\begin{array}{l}\text { Awareness } \\
\text { that men do } \\
\text { not have to } \\
\text { act tough to } \\
\text { be cool }\end{array}$ & $\begin{array}{c}\text { Awareness } \\
\text { of } \\
\text { consequence } \\
\text { of actions }\end{array}$ & $\begin{array}{l}\text { Problem } \\
\text { solving } \\
\text { skills/ } \\
\text { Helping } \\
\text { behaviors } \\
\end{array}$ \\
\hline & & & & & $\begin{array}{c}\text { Break-down of } \\
\text { traditional } \\
\text { masculine } \\
\text { stereotypes }\end{array}$ & & \\
\hline
\end{tabular}


Table 17. Qualitative Analysis: Data Responses Coded into Eight Themes

\begin{tabular}{|l|r|r|r|r|}
\hline & \multicolumn{1}{c|}{$\begin{array}{c}\text { Emotional } \\
\text { Stoicism }\end{array}$} & $\begin{array}{c}\text { Heterosexual } \\
\text { Dominance }\end{array}$ & $\begin{array}{c}\text { The Council - } \\
\text { Group Specific }\end{array}$ & $\begin{array}{c}\text { New } \\
\text { Perspective }\end{array}$ \\
\hline S8 & 34 & 2 & 1 & 61 \\
\hline S9 & 22 & 62 & 89 & 60 \\
\hline S10 & 52 & 3 & 27 & 5 \\
\hline S11 & 12 & 2 & 2 & 28 \\
\hline Total & $120(13.4 \%)$ & $69(7.7 \%)$ & $119(13.3 \%)$ & $154(17.2 \%)$ \\
\hline
\end{tabular}

\begin{tabular}{|l|r|r|r|r|}
\hline & \multicolumn{1}{|c|}{$\begin{array}{c}\text { Physical } \\
\text { Toughness }\end{array}$} & $\begin{array}{c}\text { Competition \& } \\
\text { Ambition }\end{array}$ & \multicolumn{1}{c|}{ "Man Up" } & \multicolumn{1}{c|}{ Relational } \\
\hline S8 & 25 & 14 & 49 & 129 \\
\hline S9 & 28 & 22 & 63 & 45 \\
\hline S10 & 4 & 1 & 0 & 28 \\
\hline S11 & 11 & 5 & 13 & 23 \\
\hline Total & $68(7.6 \%)$ & $42(4.7 \%)$ & $125(13.9 \%)$ & $225(25.1 \%)$ \\
\hline
\end{tabular}

Notes: Percent out of 897 data responses. Themes are not mutually exclusive within a data response. 
Figure 1. Proposed theoretical model

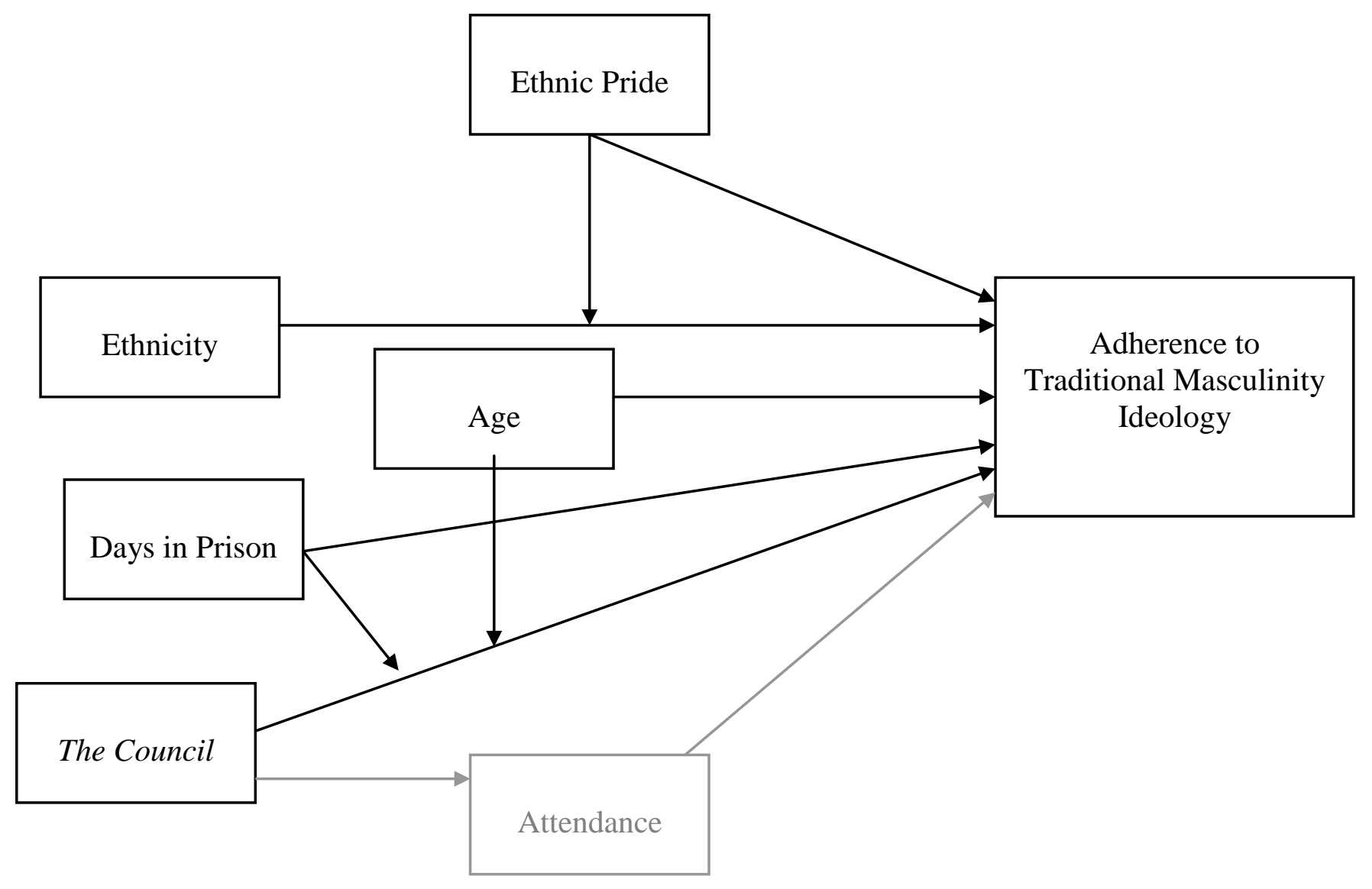


Figure 2. Qualitative Analysis: Phase 2
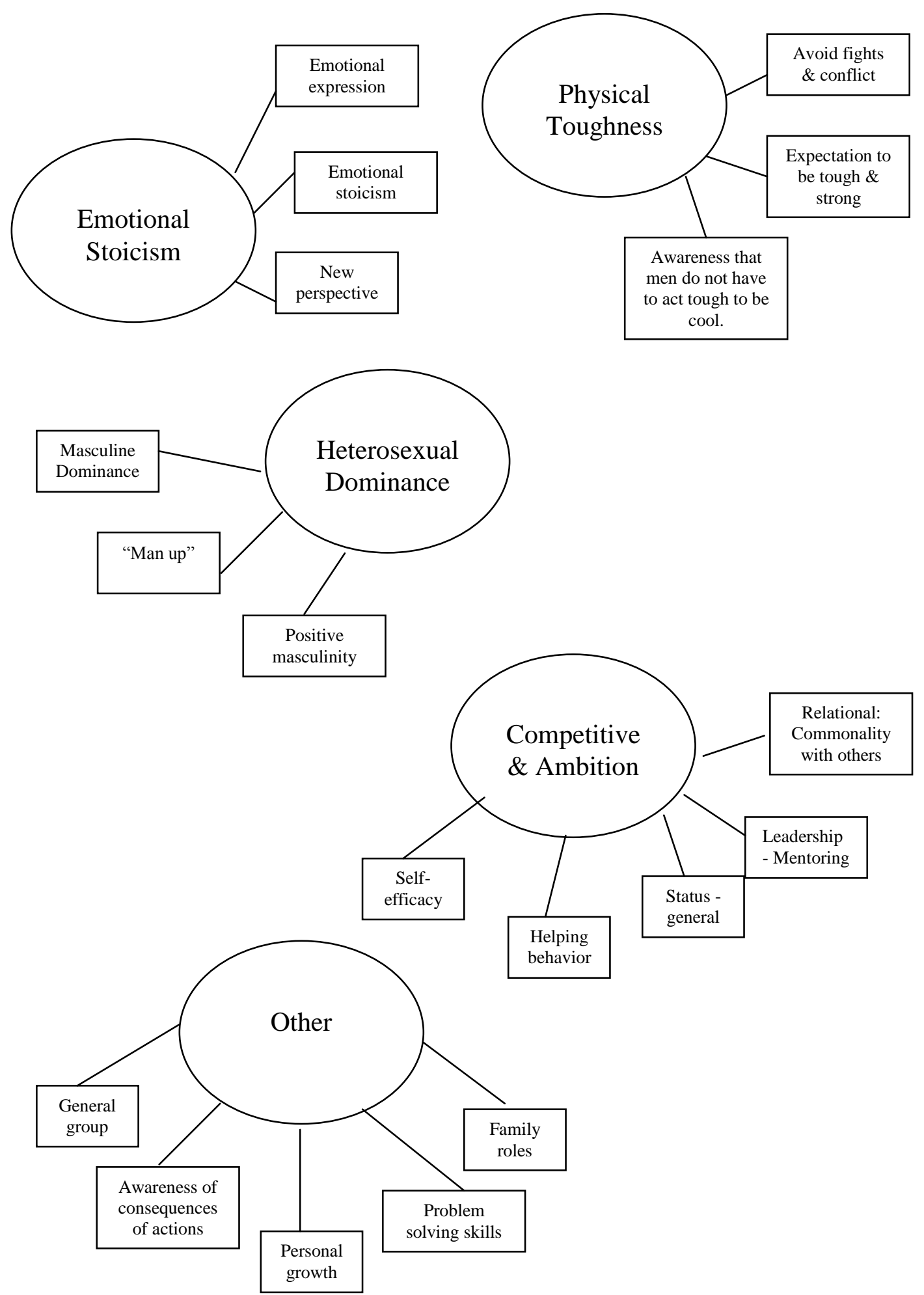
Figure 3. Qualitative Analysis: Phase 4
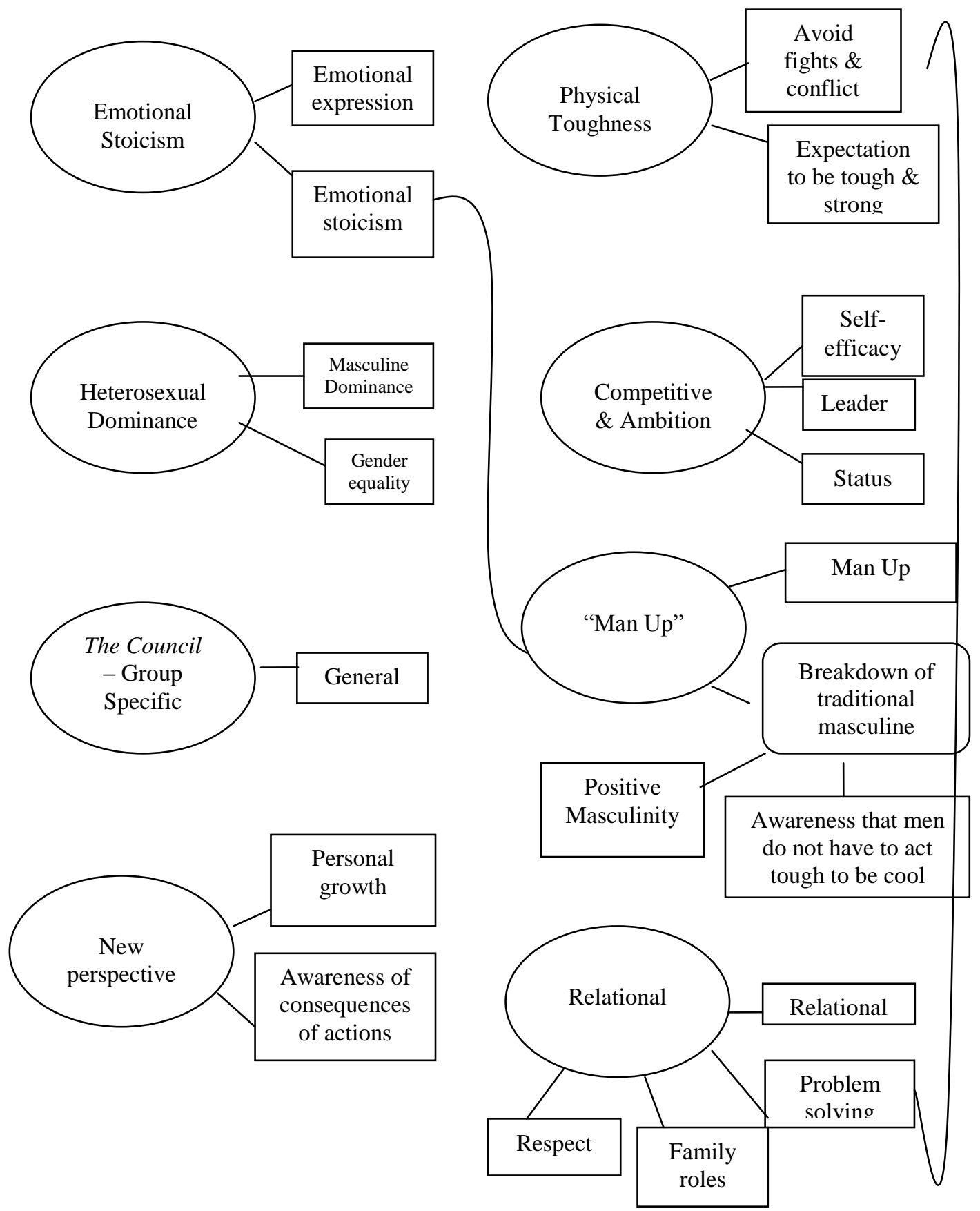
Figure 4. Qualitative Analysis: Phase 5, Renaming themes

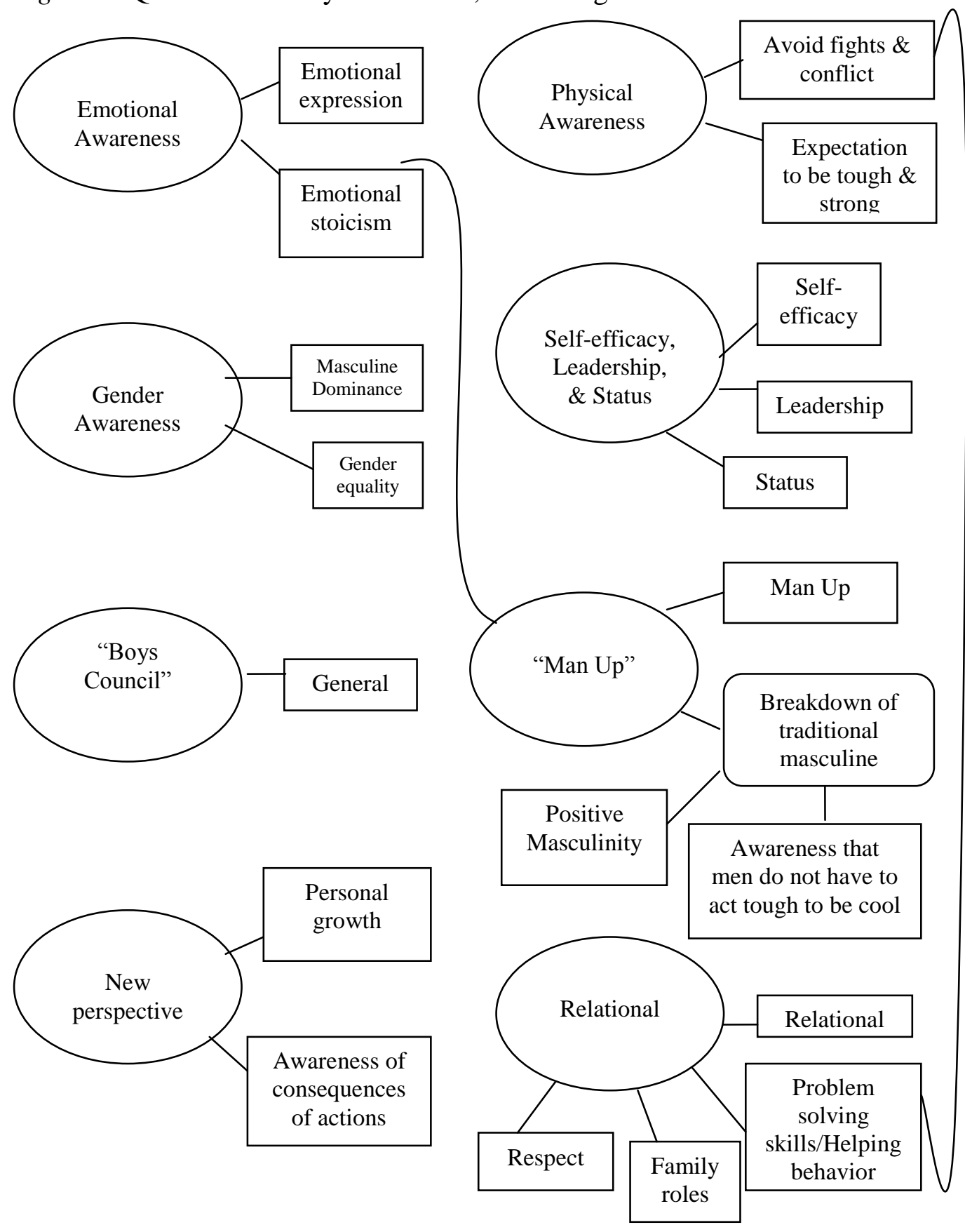


Figure 5. Control Site Growth Curve (Cuyahoga Hills)

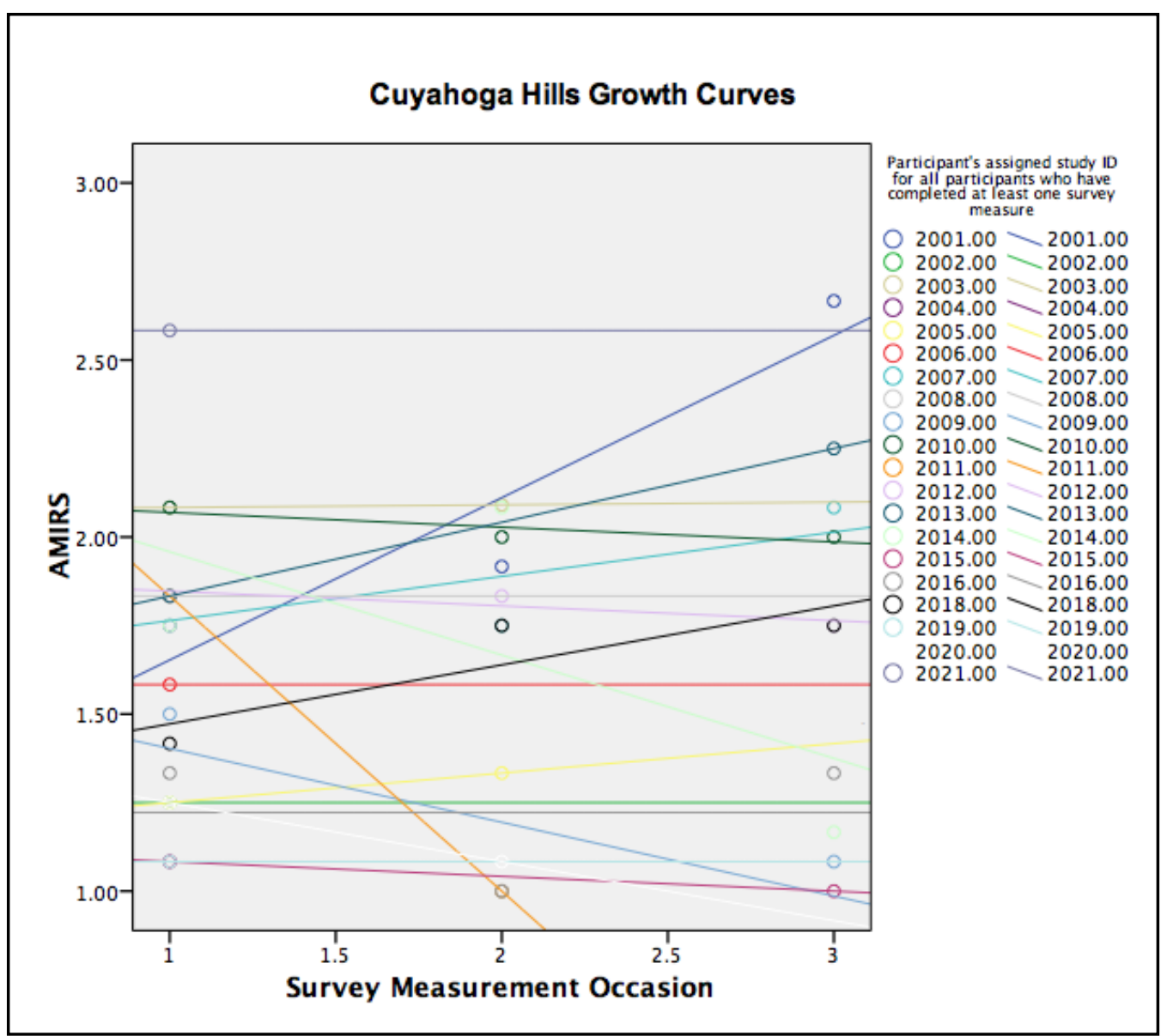


Figure 6. Control Site Growth Curve (Indian River)

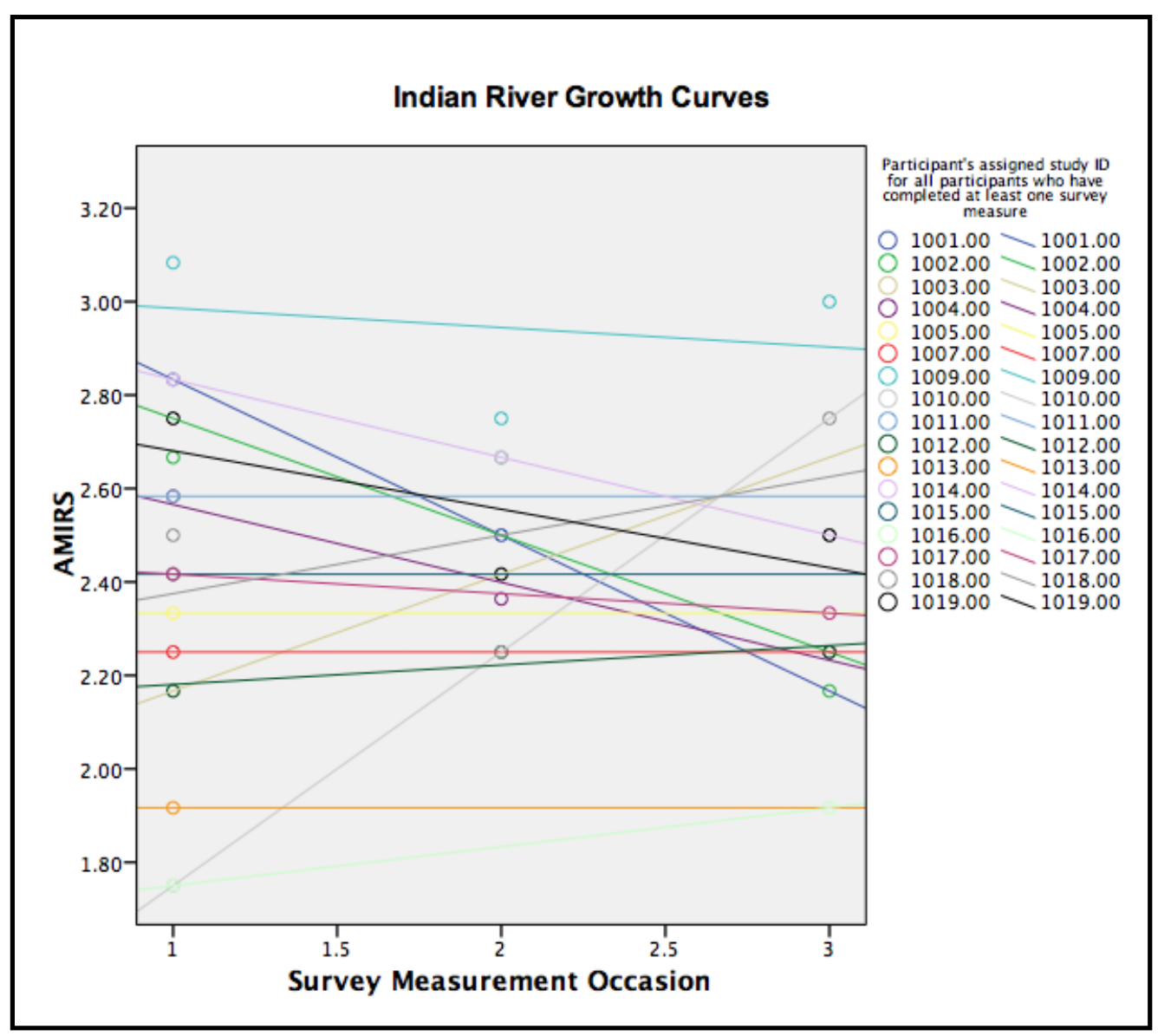


Figure 7. Experimental Site Growth Curve (Circleville)

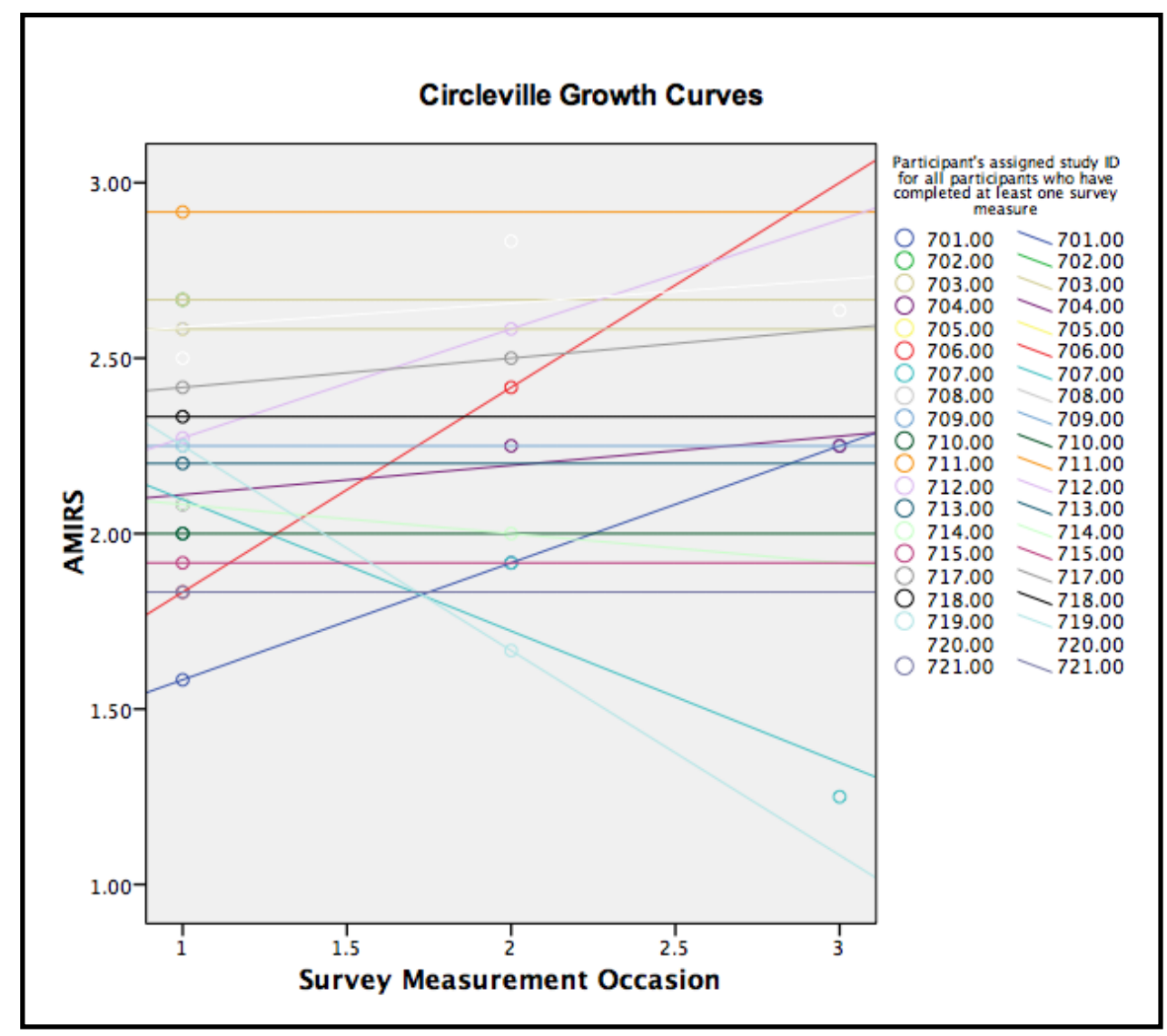


Figure 8. Experimental Site Growth Curve (Ohio River Valley)

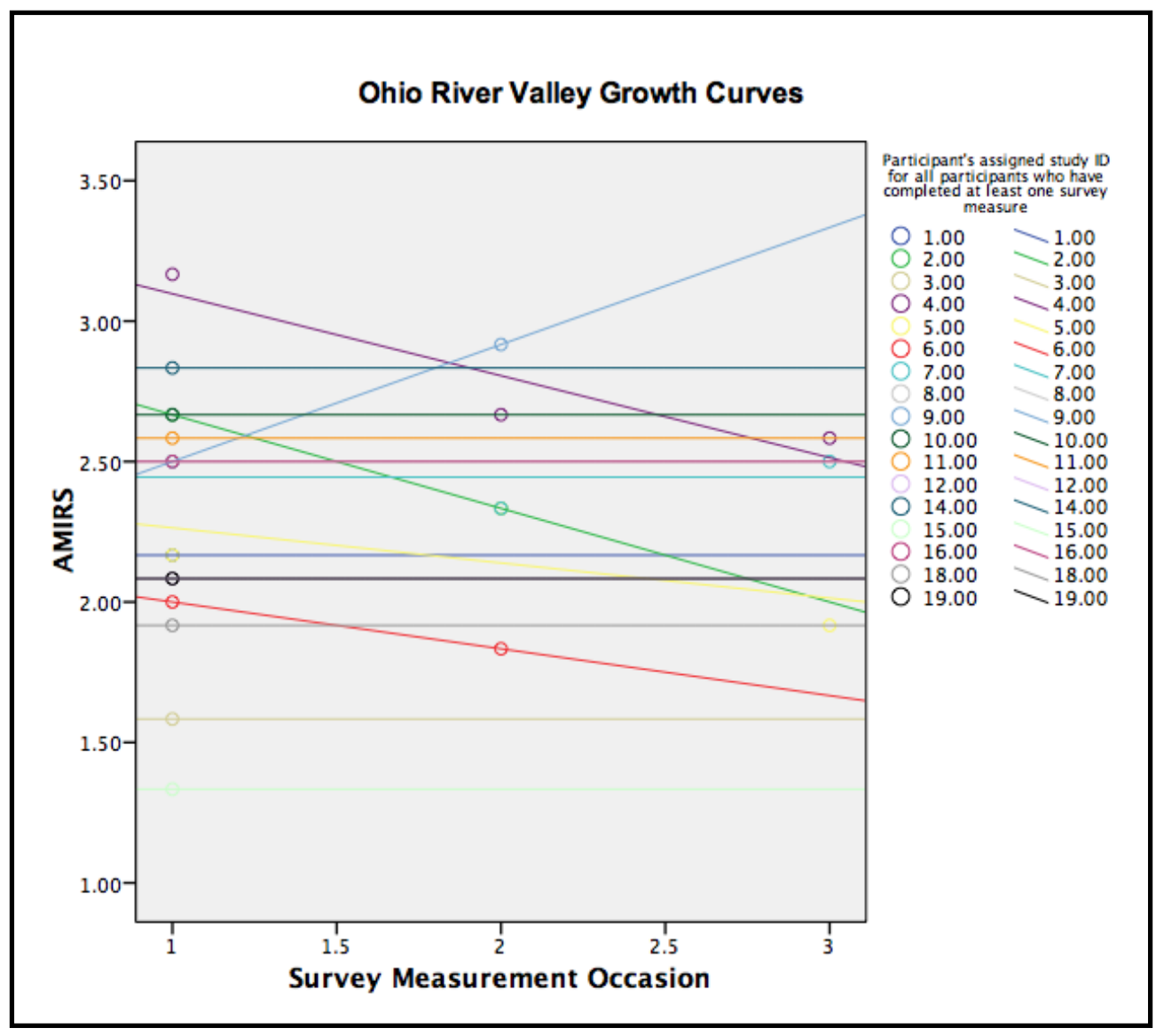


Figure 9a. Hypothesis 1a: Program Effect

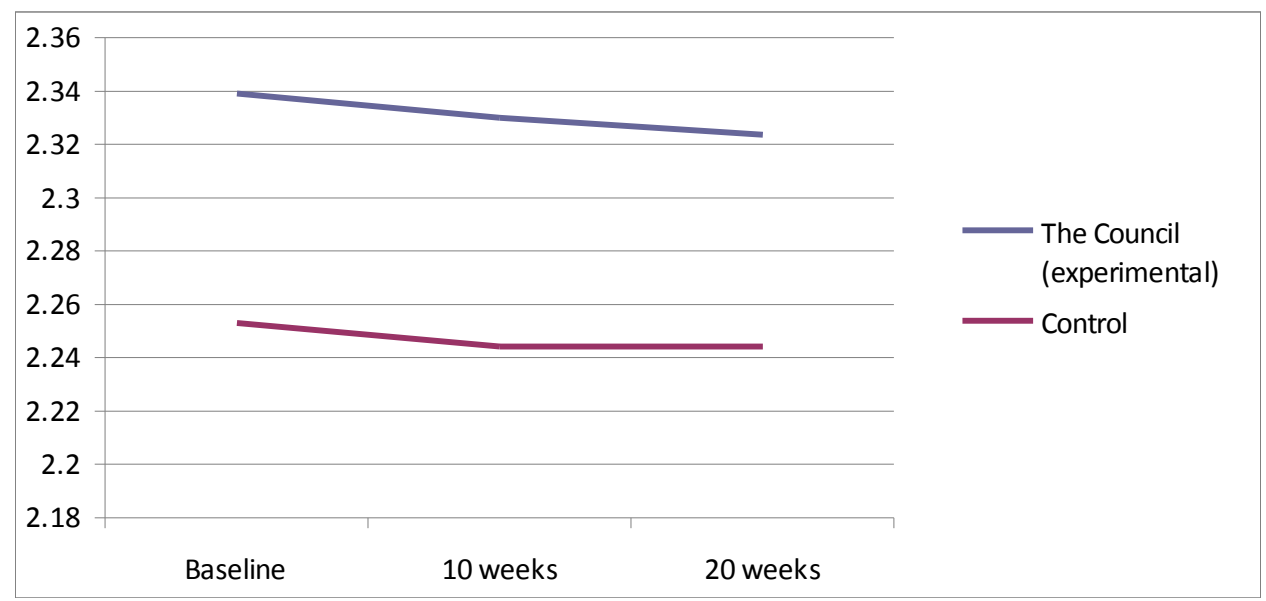

Figure 9b. Hypothesis 1b: Dosage Effect

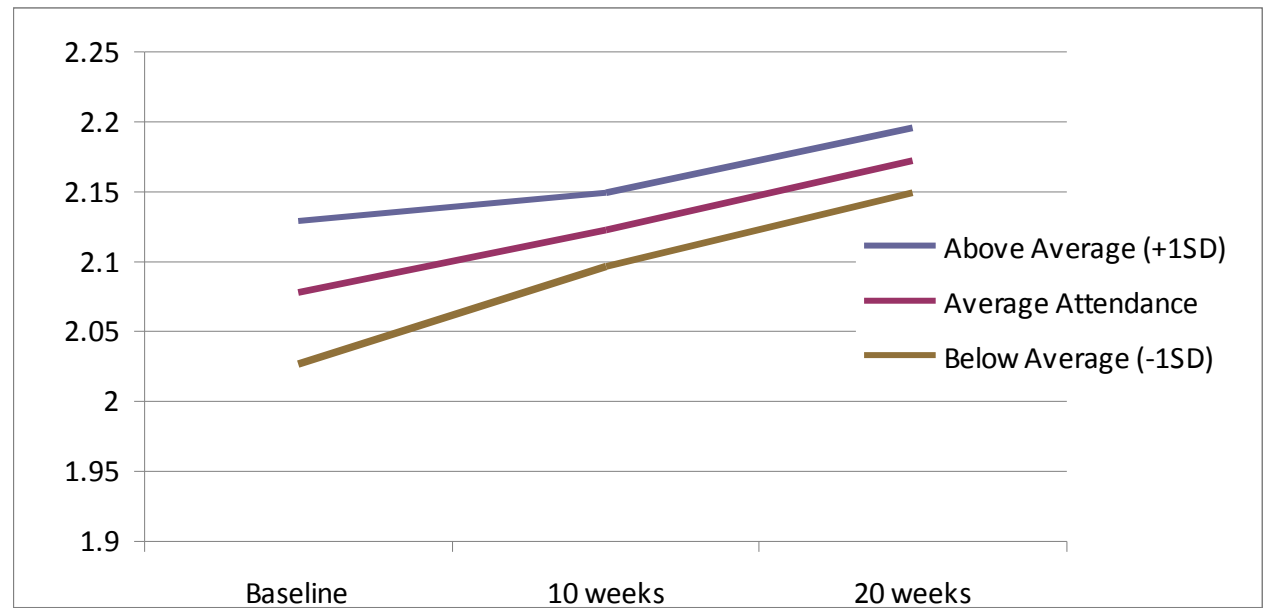


Figure 10a. Hypothesis 2a: Age and Initial Levels of Adherence

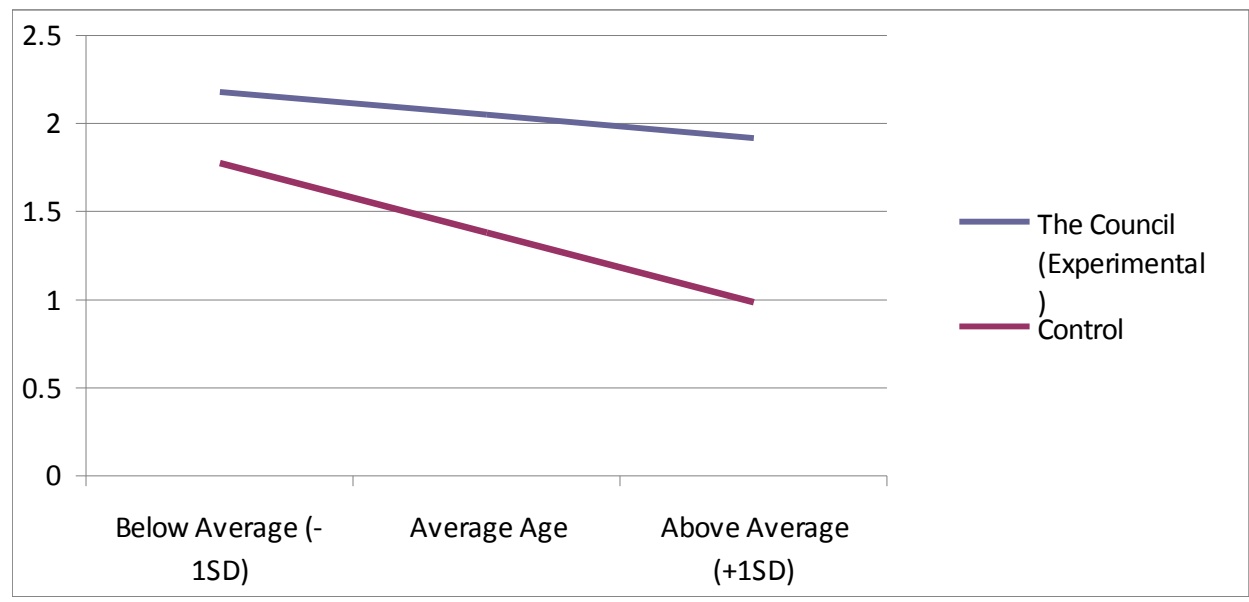

Figure 10b. Hypothesis 2b: Age and Change in Levels of Adherence

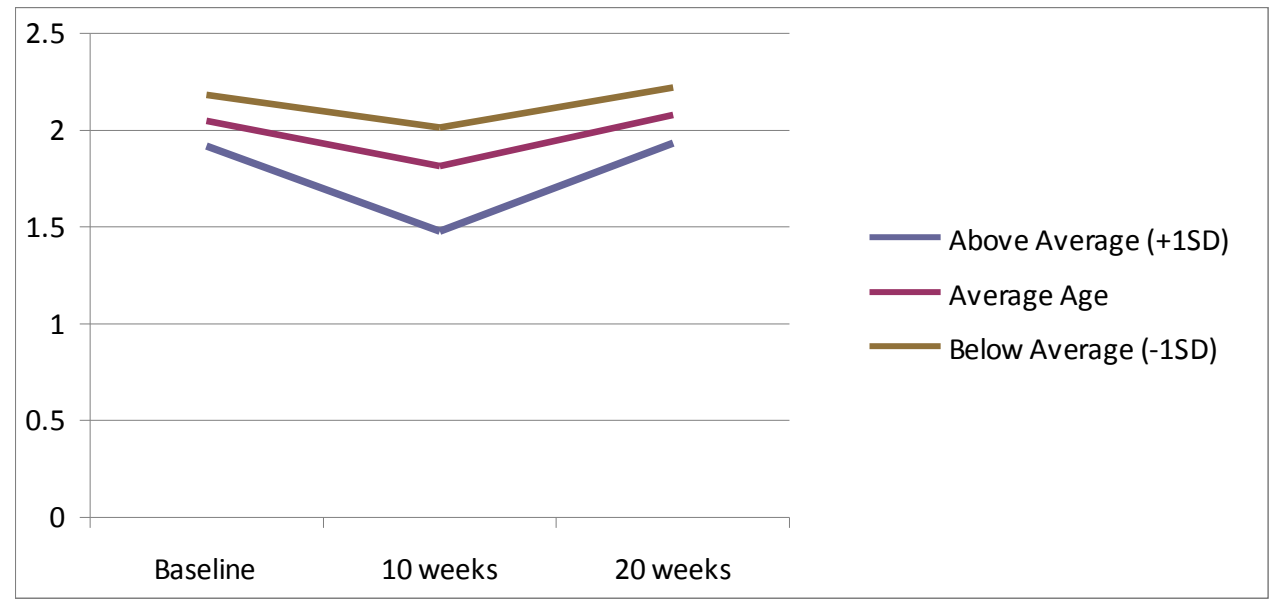


Figure 11a. Hypothesis 3a: Comparison of significant difference between African American and White

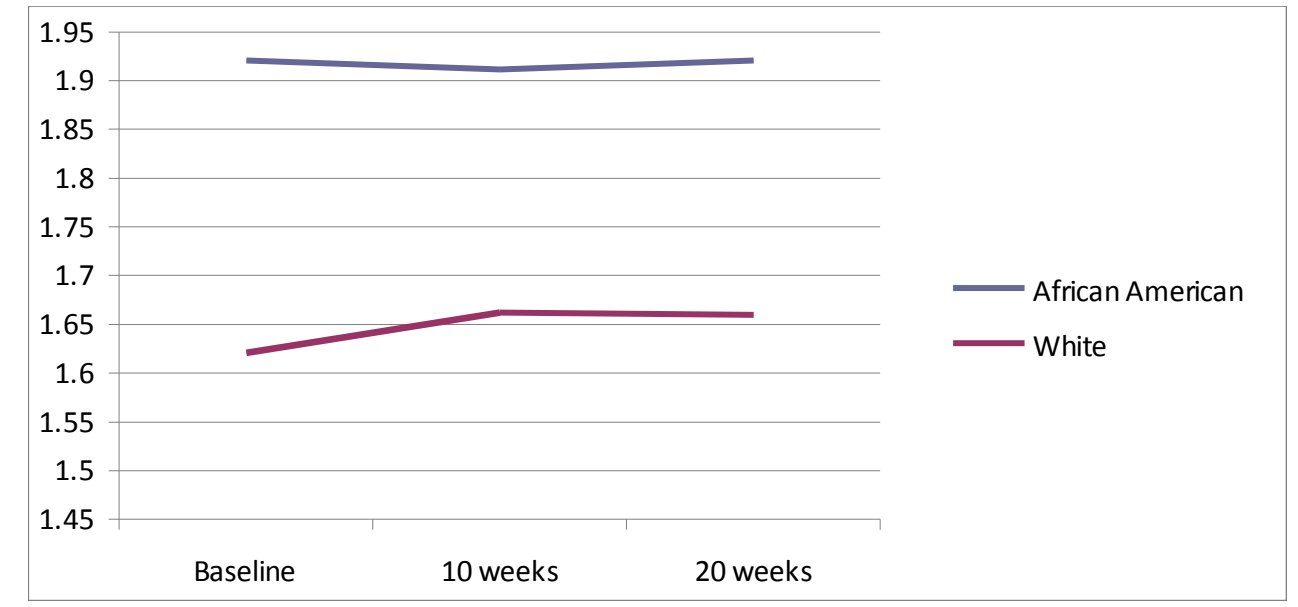


Figure 12a. Hypothesis 4a: Level of Ethnic Pride and Level of Adherence

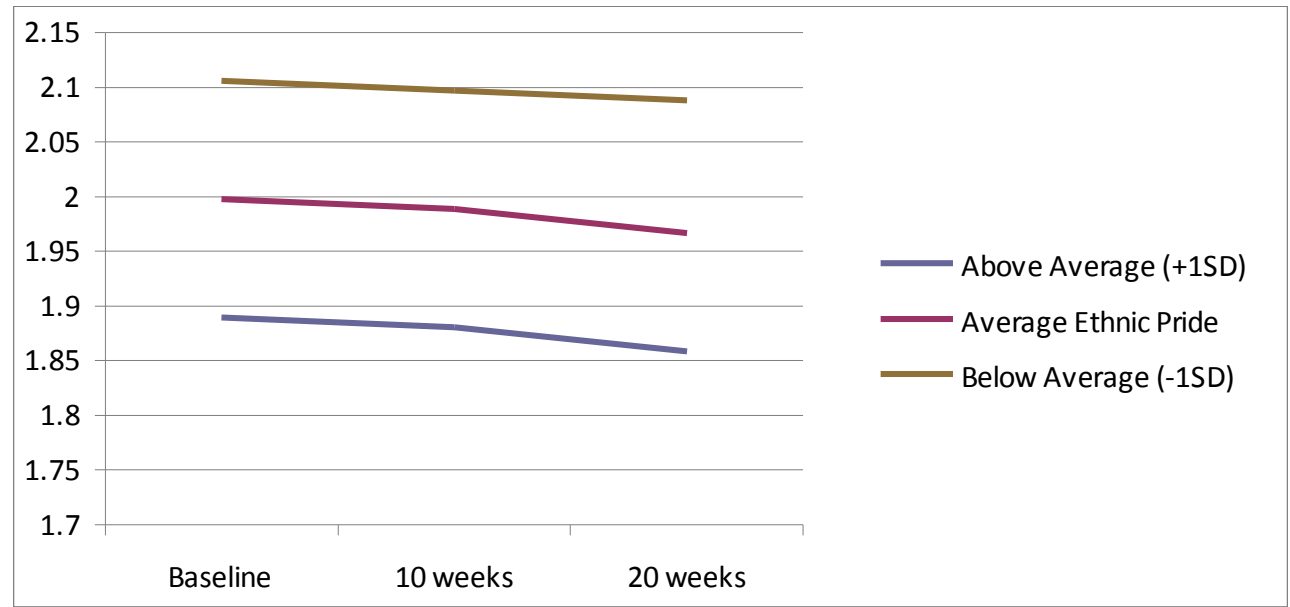

Note: Graph of African American participants (controlling for all study variables) 


\section{REFERENCES}

Abrams, L. S., Anderson-Nathe, B., \& Aguilar, J. (2008). Constructing masculinities in juvenile corrections. Men and Masculinities, 11(22), 22-41.

Abreu, J. M., Goodyear, R. K., Campos, A., \& Newcomb, M. D. (2000). Ethnic belonging and traditional masculinity ideology among African Americans, European Americans, and Latinos. Psychology of Men \& Masculinity, 1(2), 75-86.

Ackerman, P. L., \& Heggestad, E. D. (1997). Intelligence, personality, and interests: Evidence for overlapping traits. Psychological Bulletin, 121(2), 219-245.

Acock, A. (in press). What to do about missing values. APA Handbook of Research Methods in Psychology.

Altman, I., Rogoff, B. (1987). World views in psychology: Trait, interactional, organismic, and transactional perspectives. In D. Stokols I. Altman (Eds.), Handbook of Environmental Psychology. Volume 1 (pp. 7-40). New York: Wiley.

Barton, W. H. \& Butts, J. A. (2008). Building on strength: Positive youth development in Juvenile Justice programs. Chicago: Chapin Hall Center for Children at the University of Chicago.

Belknap, J. (1996). The invisible woman: Gender, crime, and justice. Cincinnati, OH: Wadsworth.

Bem, S. L. (1974). The measurement of psychological androgyny. Journal of Consulting and Clinical Psychology, 42(2), 155-162. 
Bernard, B. (2004). Resiliency: What we have learned. San Francisco, CA: WestEd. Betancourt, H., \& Lopez, S. R. (1993). The study of culture, ethnicity, and race in American psychology. American Psychologist, 48(6), 629-637.

Blazina, C., Eddins, R., Burridge, A., \& Settle, A. G. (2007). The relationship between masculine ideology, loneliness, and separation-individuation difficulties. The Journal of Men's Studies, 15(1), 101-109.

Blazina, C., \& Marks, L. I. (2001). College men's affective reactions to individual therapy, psychoeducational workshops, and men's support group brochures: The influence of gender-role conflict and power dynamics upon help seeking attitudes. Psychotherapy, 38(3), 297-305.

Blazina, C., Pisecco, S., \& O'Neil, J. M. (2005). An adaptation of the gender role conflict scale for adolescents: Psychometric issues and correlates with psychological distress. Psychology of Men \& Masculinity, 6(1), 39-45.

Blazina, C., \& Watkins, Jr., E. C. (2000). Separation/individuation, parental attachment, and male gender role conflict: Attitudes toward the feminine and the fragile masculine self. Psychology of Men \& Masculinity, 1(2), 126-132.

Bortner, M. A., \& Williams, L. M. (1997). Youth in Prison: We the People of Unit Four. New York and London: Routledge.

Bosworth, K. \& Espelage, D. (1995). Ethnic Identity - Teen Conflict. In Dahlberg, L.L., Toal, S.B., Swahn, M., Behrens, C.B. (2005). Measuring ViolenceRelated Attitudes, Behaviors, and Influences Among Youths: A Compendium 
of Assessment Tools, 2nd ed., Atlanta, GA: Centers for Disease Control and Prevention, National Center for Injury Prevention and Control.

Brannon, R. (1976). The male sex role: Our culture's blueprint for manhood and what it's done for us lately. In D. David \& R. Brannon (Eds), The forty-nine percent majority: The male sex role (pp. 1-48). Reading, MA: AddisonWesley.

Brannon, R. (1985). Dimensions of the male sex role in America. In Beyond sex roles (2nd ed., pp. 296-316). New York: West.

Brannon R. \& Juni, S. (1984). A scale for measuring attitudes toward masculinity. JSAS Psychological Documents, 14, 6-7.

Braun, V., \& Clarke, V. (2006). Using thematic analysis in psychology. Qualitative Research in Psychology, 3, 77-101.

Bronfenbrenner, U. (1979). The Ecology of Human Development: Experiments by Nature and Design. Cambridge, MA: Harvard University Press.

Brown, D. A. (1958). Sex-role development in a changing culture. Psychological Bulletin, 55(4), 232-242.

Centers for Disease Control and Prevention (2010). Youth violence: Facts at a glance. Retrieved January 6, 2011, from http://www.cdc.gov/violenceprevention/youthviolence/

Centers for Disease Control and Prevention (2011). Youth violence: National statistics: Violent crime arrests. Retrieved January 6, 2011, from http://www.cdc.gov/violenceprevention/youthviolence/ 
Cesaroni, C., \& Alvi, S. (2010). Masculinity and resistance in adolescent caceral settings. Canadian Journal of Criminology and Criminal Justice, 52(3), 303320.

Chu, J. Y., Porche, M. V., \& Tolman, D. L. (2005). The adolescent masculinity ideology in relationship scale. Men and Masculinities, 8(1), 93-115.

Cobb, N. J. (1992). Adolescence: Continuity, Change, and Diversity. Mountain View, CA: Mayfield.

Cohen, J. (1988). Statistical power analysis for the behavioral sciences ( $2^{\text {nd }}$ Edition). Hillsdale: Lawrence Erlbaum Associates.

Cohn, A. M. \& Zeichner (2006). Effects of masculine identity and gender role stress on aggression in men. Psychology of Men and Masculinity, 7, 179-190.

Cole, J. C. (2008). How to deal with missing data: Conceptual overview and details for implementing two modern methods. In Best Practices in Quantitative Methods (Edited by Jason W. Osborne., pp. 214-238). Thousand Oaks, CA: Sage Publications, Inc.

Connell, R. W. (1987). Gender and Power. Cambridge: Polity Press

Connell, R. W. (1992). A very straight gay: Masculinity, homosexual experience, and the dynamics of gender. American Sociological Review, 57, 735-751.

Connell, R. W. (1995). Masculinities. Berkeley: University of California Press.

Connell, R. W., \& Messerschmidt, J. W. (2005). Hegemonic masculinity: Rethinking the concept. Gender \& Society, 19(6), 829-859. 
Cournoyer, R. J., \& Mahalik, J. R. (1995). Cross-sectional study of gender role conflict examining college-aged and middle-aged men. Journal of Counseling Psychology, 42, 11-19.

Doyle, J. A. (1995). The Male Experience, $3^{\text {rd }}$ Ed. Madison: WI, Brown \& Benchmark.

Ely, M., Anzul, M., Friedman, T., Garner, D., \& McCormack Steinmetz, A. (1991). Doing Qualitative Research: Cirlces within Circles. London: Falmer Press.

Eisler, R. M., \& Skidmore, J. R. (1987). Masculine gender role stress: Scale development and component factors in the appraisal of stressful situations. Behavior Modification, 11, 123-136.

Enders, C. K. \& Tofighi, D. (2007). Centering predictor variables in cross-sectional multilevel models: A new look at an old issue. Psychological Methods, 12, 121-138.

Erickson, E. H. (1950). Childhood and Society. New York, NY: Norton.

Erickson, E. H. (1968). Identity: Youth and Crisis. New York, NY: Norton.

Fowler, F. J. Jr. (1995). Improving survey questions: Design and evaluation. Thousand Oaks, CA: Sage Publications.

Franklin II, C. W. (1984). Black male-Black female conflict: Individually caused and culturally nurtured. Journal of Black Studies, 15(2), 139-154.

Freeman, C. E. (2006). Trends in Educational Equity of Girls \& Women: 2004. US Department of Education, National Center for Education Statistics. Washington, DC: US Government Printing Office. 
Galambos, N. L, Peterson, A. C., Richards, M., \& Gitelson, B. I. (1985). The attitudes towards women scale for adolescents (AWAS): A study of reliability and validity. Sex Roles, 13, 343-356.

Garnets, L., \& Pleck, J. H. (1979). Sex role identity, androgyny, and sex role transcendence: A sex role strain analysis. Psychology of Women Quarterly, $3(3), 270-283$.

George, D. \& Mallery, P. (2003). SPSS for Windows step by step: A simple guide and reference. 11.0 update ( $4^{\text {th }}$ edition). Boston, MA: Allyn \& Bacon.

Gerschick, T. J. \& Miller, A. S. (1997). Gender identities at the crossroads of masculinity and physical disability. In M. M. Gergen \& S. N. Davis (Eds.) Toward a New Psychology of Gender, New York, NY: Routledge.

Greene, J. P. \& Winters, M. A. (April 2006). Leaving boys behind: Public high school graduation rates. Manhattan Institute for Policy Research, Civic Report, No. 48.

Groves, R. M., Fowler, F. J., Couper, M. P., Lepkowski, J. M., Singer, E. \& Tourangeau, R. (2009). Survey methodology (2nd edition). Hoboken, NJ: Wiley.

Guilford, J. P. \& Zimmerman, W. S. (1956). The Guilford-Zimmerman Temperament Survey. Beverly Hills, CA: Sheridan Supply.

Hall, G. S. (1904). Adolescence: Its psychology and its relation to physiology, anthropology, sociology, sex, crime, religion, and education. Englewood Cliffs, NJ: Prentice-Hall. 
Havighurst, R. J. (1972). Developmental tasks and education. New York: David McKay.

Higgins, T. E. (1987). Self-discrepancy: A theory relating self and affect. Psychological Review, 94(3), 319-340.

Hoffman, R. M. (2001). The measurement of masculinity and femininity: Historical perspective and implications for counseling. Journal of Counseling \& Development, 79, 472-485.

Hossfeld, B., Gibraltarik, R., Bowers, M., and Taormina, G. (2008). Boys Council Facilitator Manual: Promoting Resiliency in Adolescent Boys. Boys Council, A Division of GCA/Tides: Cotati, CA

Hox, J. J. (2010). Multilevel Analysis: Techniques and Applications. New York: Routledge: Taylor \& Francis Group.

Inhelder, B., \& Piaget, J. (1958). The Growth of Logical Thinking From Childhood to Adolescence. New York, NY: Basic Books.

Jakupcak, M., Lisak, D., \& Roemer, L. (2002). The role of masculine ideology and masculine gender role stress in men's perpetration of relationship violence. Psychology of Men \& Masculinity, 3(2), 97-106.

Jewkes, Y. (2005). Men behind bars: "Doing" masculinity as an adaptation to imprisonment. Men and Masculinities, 8(1), 44-63.

Kelly, G. F. (2004). Sexuality Today: The Human Perspective. Boston, MA: McGraw-Hill. 
Kilmartin, M. S. (2007). The masculine self(3rd ed.). Cornwall-on-Hudson, NY: Sloan Publishing.

Kimmel, M. S. (2007). Masculinity as homophobia: Fear, shame, and silence in the construction of gender identity. In N. Cook (Eds), Gender Relations in Global Perspective: Essential Readings. Canadian Scholars Press.

Kimmel, M. S., \& Mahler, M. (2003). Adolescent masculinity, homophobia, and violence. American Behavioral Scientist, 45, 1439-1458.

Kivel, P. (2007). The Act-Like-a-Man Box. In Men's Lives, Kimmel, M.S. \& Messner, M.A. (7th ed., pp. 148-150). Boston: Pearson.

Levant, R. F., Hirsh, L., Celentano, E., Cozza, T., Hill, S., MacEachern, M., Marty, N., \& Schnedeker, J. (1992). The male role: An investigation of norms and stereotypes. Journal of Mental Health Counseling, 14, 325-337.

Levant, R. F., Graef, S. T., Smalley, B. K., Williams, C., McMillan, N. (2008). Evaluation of the psychometric properties of the Male Role Norms Inventory-Adolescent (MRNI-A). Thymos: Journal of Boyhood Studies, 2(1), 45-59.

Levant, R. F. \& Majors, R. G. (1997). An investigation into variations in the construction of the male gender role among young African American and European American women and men. Journal of Gender, Culture, and Health, 2, 33-43.

Levant, R. F., Majors, R. G., \& Kelley, M. L. (1998). Masculinity ideology among young African American and European American women and men in 
different regions of the United States. Cultural Diversity and Mental Health, 4(3), 227-236.

Levant, R. F., \& Richmond, K. (2007). A review of research on masculinity ideologies using the male role norms inventory. The Journal of Men's Studies, 15(2), 130-146.

Levant, R. F., Richmond, K., Majors, R. G., Inclan, J. E., Rosello, J. M., Heesacker, M., ... Seilers, A. (2003). A multicultural investigation of masculinity ideology and alexithymia. Psychology of Men \& Masculinity, 4(2), 91-99.

Liebling, A. (1999). Prison suicide and prison coping. In Prisons: Crime and Justice - A Review of Research (In M. H. Tonry and J. Peterselia (eds).). Chicago, IL: University of Chicago Press.

Lincoln, Y. S. \& Guba, E. G. (1985). Naturalistic inquiry. Beverly Hills, CA: Sage Publications, Inc.

Lippa, R. A. (2002). Gender, Nature, and Nurture. Mahwah, New Jersey: Lawrence Erlbaum Associates, Inc.

Liu, X., Spybrook, J., Congdon, R., Martinez, A., \& Raudenbush, S. (2009). Optimal Design for Multi-level and Longitudinal Research software (Version 2.0). Available from http://www.wtgrantfoundation.org/resources/overview/research_tools

Lutze, F. E. \& Murphy, D. W. (1999). Ultramasculine prison environments and inmates' adjustment: It's time to move beyond the "boys will be boys" paradigm. Justice Quarterly, 16(4), 709-733. 
Majors, R. G., \& Billson, J. M. (1992). Cool Pose: The Dilemmas of Black Manhood in America. New York, NY: Lexington Books.

Mankowski, E. S., \& Maton, K. I. (2010). A community psychology of men and masculinity: Historical and conceptual review. American Journal of Community Psychology, 45(1-2), 73-86.

Merrick, E. (1999). An exploration of quality in qualitative research: Are reliability and validity relevant? In M. Kopala \& L.A. Suzuki (Eds.). Using Qualitative Methods in Psychology (pp. 25-36). Thousand Oaks, CA: Sage.

Messerschmidt, J. W. (1993). Masculinities and Crime: Critique and Reconceptualization of Theory. Lanham, MD: Rowman \& Littlefield Publishers, Inc.

Miller, J. B. (1991). The development of women's sense of self. In J. Jordan et al. Women's Growth in connection: Writings from the Stone Center. New York, NY: Guilford Press.

Morgan, D. L. (1998). Practical strategies for combining qualitative and quantitative methods: Applications to health research. Qualitative Health Research, 3, 362-376.

Nunnally, J. C. (1978). Psychometric Theory (2nd ed.). New York, NY: McGrawHill.

O’Neil, J. M. (1981). Male sex-role conflict, sexism, and masculinity: Implications for men, women, and the counseling psychologist. The Counseling Psychologist, 9, 61-80. 
O'Neil, J. M. (1990). Assessing men's gender role conflict. In Men in conflict: Problem solving strategies and interventions (D. Moore \& F. Laefgren (Eds)., pp. 23-38). Alexandria VA: American Association for Counseling and Development.

O'Neil, J. M. (2008). Summarizing 25 years of research on men's gender role conflict using the gender role conflict scale: New research paradigms and clinical implications. The Counseling Psychologist, 36, 358-445.

O’Neil, J. M., Helms, B. J., Gable, R. K., David, L. and Wrightsman, L. S. (1986). Gender-Role Conflict Scale: College men's fear of femininity. Sex Roles, $14,335-350$.

O'Neil, J. M., \& Lujan, M. L. (2009). Preventing boys' problems in school through psychoeducational programming: A call to action. Psychology in the Schools, 46(3), 257-266.

Peterson, A. C. (1988). Adolescent Development. Annual Review of Psychology, 30(1), 583-607.

Phinney, J. (1992). The multigroup ethnic identity measure: A new scale for use with adolescents and young adults from diverse groups. Journal of Adolescent Research, 7, 156-176.

Phinney, J. S. (1996). When we talk about American ethnic groups, what do we mean? American Psychologist, 51(9), 918-927. 
Pleck, J. H. (1987). The theory of male sex-role identity: Its rise and fall, 1936 to the present. In H. Brod (Ed.), The making of masculinities: The new men's studies (pp. 11-32). New York: Basic Books.

Pleck, J. H., Sonenstein, F. L., \& Ku, L. C. (1993). Masculinity ideology and its correlates. In Gender Issues in Contemporary Society, The Claremont Symposium on Applied Social Psychology (Stuart Oskamp and Mark Costanzo., pp. 85-110). Newbury Park, CA: Sage Publications.

Pleck, J. H., Sonenstein, F. L., \& Ku, L. C. (1994). Attitudes toward male roles: A discriminant validity analysis. Sex Roles, 30, 481-501.

Pollack, W. S. (1999). Real boys: Rescuing our sons from the myth of boyhood. New York: Henry Holt \& Company.

Pollack, W. S. (2000). Real boys' voices. New York: Random House.

Pollack, W. S. (2006a). The "war" for boys: Hearing "real boys" voices, healing their pain. Professional Psychology: Research and Practice, 37(2), 190-195.

Pollack, W. S. (2006b). Male adolescent rites of passage: Positive visions of multiple developmental pathways. Annals of the New York Academy of Sciences, 1036(1), 141-151.

Quinn, M. M., Rutherford, R. B., \& Leone, P. E. (2001,February). Students with disabilities in correctional facilities. Arlington, VA: Eric Clearinghouse on Disabilities and Gifted Education. Retrieved January 15, 2012, from http://ericec.org/digests/e621.html 
Rando, R. A., Rogers, J. R., \& Brittan-Powell, C. (1998). Gender role conflict in college men's sexually aggressive attitudes and behavior. Journal of Mental Health Counseling, 20, 359-369.

Resnick, M. D., Bearman, P. S., Blum, W. M., Bauman, K. E., Harris, K. M., Jones, H., ... Udry, R. (1997). Protecting adolescents from harm: Findings from the national longitudinal study on adolescent health. Journal of the American Medical Association, 278(10), 823-832.

Ritchie, J. (2003). The applications of qualitative methods to social research. In J. Ritchie \& J. Lewis (Eds.) Qualitative Research Practice: A Guide for Social Science Students and Researchers (pp. 219-262). Thousand Oaks, CA: Sage Publications Inc.

Ritchie, J., Spencer, L., \& O’Connor, W. (2003). Carrying out qualitative analysis. In J. Ritchie \& J. Lewis (Eds.) Qualitative Research Practice: A Guide for Social Science Students and Researchers (pp. 219-262). Thousand Oaks, CA: Sage Publications Inc.

Rosenberg, M. (1965) Society and the adolescent self image. Princeton, NJ: Princeton University Press.

Rubin, D. B. (1976). Inference and missing data. Biometrika, 63, 581-592.

Sabo, D., Kupers, T. A., \& London, W. (2001). Gender and the politics of punishment. In Prison Masculinities (In D. Sabo, T. A. Kupers, \& W. London (eds).). Philadelphia, PA: Temple University Press. 
Saez, P. A., Casado, A., \& Wade, J. C. (2009). Factors influencing masculinity ideology among Latino men. Journal of Men's Studies, 17(2), 116-128.

Schafer, J. (2005). Missing Data in Longitudinal Studies: A Review. Presented at the AAPS, Nashville, TN.

Schnyder, D. M. (2010). First strike: The effect of the prison regime education and black masculinity in Los Angeles County, California. Dissertation Abstracts International Section A: Humanities and Social Sciences. 70, 8-A, 3066.

Segall, M. H. (1984). More than we need to know about culture, but are afraid not to ask. Journal of Cross-Cultural Psychology, 15, 153-162.

Senn, C. Y., Desmarias, S., Verberg, N., \& Wood, E. (2000). Predicating coercive sexual behavior across the lifespan in a random sample of Canadian men. Journal of Social and Personal Relationships, 17, 95-113.

Smiler, A. P. (2004). Thirty years after the discovery of gender: Psychological concepts and measures of masculinity. Sex Roles, 50(1/2), 15-26.

Snell, W. E. (1989). Development and validation of the masculine behavior scale: A measure of behaviors stereotypically attributed to males vs. females. Sex Roles, 21(11-12), 749-767.

Snell, W. E., Belk, S. S., \& Hawkins, R. C. II (1986). The stereotypes about male sexuality scale (SAMSS): Components, correlates, antecedents, consequences, and counselor bias. Social and Behavioral Sciences Documents, 16(9). 
Snickmund, M., Snyder, H., \& Poe-Yamagata, E. (1997). Juvenile Offenders and Victims: 1997 Update on Violence -- Statistics Summary. Washington, D.C.: National Center for Juvenile Justice: OJJDP.

Spence, J. T. \& Helmrich, R. L. (1978). Masculinity and femininity: Their psychological dimensions, correlates, and antecedents. Austin: University of Texas Press.

Spencer, L., Ritchie, J., \& O’Connor, W. (2003). Analysis: Practices, principles, and processes. In J. Ritchie \& J. Lewis (Eds.) Qualitative Research Practice: A Guide for Social Science Students and Researchers (pp. 199-218). Thousand Oaks, CA: Sage Publications Inc.

Spybrook, J., Raudenbush, S. W., Congdon, R., Martinzes, A. (2009). Optimal design for longitudinal and multilevel research: Documentation for the “Optimal Design” software. Retrieved March 16, 2011, from http://www.wtgrantfdn.org/resources/overview/research_tools/research_tools

Steinberg, L., \& Sheffield Morris, A. (2001). Adolescent development. Annual Review of Psychology, 52(1), 83-110.

Terman, L. \& Miles, C. (1936). Sex and personality: Studies in masculinity and femininity. New York: McGraw-Hill.

Thompson, JR., E. H. \& Pleck, J. H. (1986). The structure of male role norms. American Behavioral Scientist, 29, 531-543.

Thompson, JR., E. H., \& Pleck, J. H. (1995). Masculine ideologies: A review of research instrumentation on men and masculinities. In A New Psychology of 
Men (R. F. Levant \& W. S. Pollack., pp. 129-163). New York, NY: Basic Books.

Trickett, E. (1996). A future for community psychology: The contexts of diversity of the diversity of contexts. American Journal of Community Psychology, 24, 209-234.

Urban Dictionary (1999-2011). Man up. In Urban Dictionary. Retrieved November 19, 2011, from http://www.urbandictionary.com/define.php?term=man\%20up .

Wade, J. C. (2008a). Traditional masculinity and African American men's healthrelated attitudes and behaviors. American Journal of Men's Health, 3, 165173.

Wade, J. C. (2008b). Masculinity ideology, male reference group identity dependence, and African American men's health-related attitudes. Psychology of Men \& Masculinity, 9(1), 5-16.

Watts, Jr., R. H., \& Borders, D. L. (2005). Boys' perceptions of the male role: Understanding gender role conflict in adolescent males. The Journal of Men's Studies, 13(2), 267-280.

Zuckerman, M. (1990). Some dubios premises in research and theory on racial differences: Scientific, social, and ethnical issues. American Psychologist, 45(1), 1297-1303. 


\author{
APPENDIX A \\ Memorandum of Understanding
}

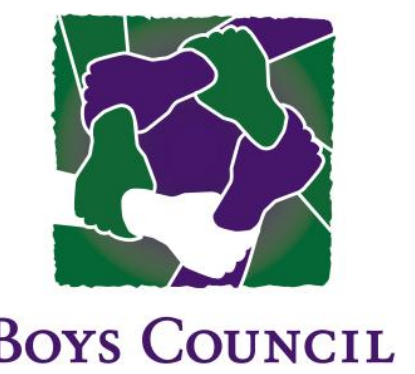

\title{
MEMORANDUM OF UNDERSTANDING
}

DATE: January 14, 2008

TO: $\quad$ Eric Mankowski, Associate Professor, Applied Social \& Community

Psychology

Department of Psychology, 317 Cramer Hall, Portland State University, P.O. Box 751, Portland, OR 97207-0751

FR: $\quad$ Beth Hossfeld, MFT, Associate Director Boys Council, A Division of GCA/Tides, $\quad 458$ Christensen Lane, Cotati, CA 94931

\section{RE: $\quad$ Boys Council Pilot Study Collaboration}

This memo is to confirm the understanding between the Department of Psychology, Portland State University (PSU)and Boys Council, A Division of GCA/Tides, (BC) to collaborate in the Boys Council Pilot Study project of 2008.

\section{DeP'T of Psychology, Portland State University agrees to:}

- Read the Boys Council Evaluation Packet, provide any recommendations, and submit to the departmental IRB for approval to launch study asap, for late Winter - Spring 2008 study;

- Identify and assign graduate and/or undergraduate student(s) who have an interest and ability in the Boys Council Pilot Study, to gather and analyze outcome survey data from collaborating organizations that have agreed to pilot the Boys Council program and administer surveys this Spring, 20008.

- Students will collect data electronically from participating organizations, OR, may provide direct data entry where organizations are not able to provide data entry; by end of May, 2008. 
- Provide collective pre- and post- survey outcome data analysis in a report to Boys Council, A Division of GCA/Tides by mid-summer, 2008.

- Explore and consider opportunities for further evaluation collaborations with

$\mathrm{BC}$, following initial pilot study, based on experience, goals, and needs of both parties.

\section{Boys COUNCIL, A DIVISION OF GCA/TIDES, AGREES TO:}

- Provide 1 Full Set of 3 Activity Boys Council Facilitator Activity Guides to PSU:

Growing Healthy, Going Strong, for ages 9-14

Standing Together: A Boys Council Journey Into Respect, for ages 9-14

Living A Legacy: A Boys Council Rite of Passage, for ages 13 - 18 years

- Provide a Boys Council Evaluation Packet including the Boys Council Survey, Participant and Parental/Guardian Consent Form, an informational sheet, instructional steps to administer the survey, and tips for facilitators for approval of packet by PSU evaluation team and for use by all collaborating organizations who will administer surveys and collect data;

- Provide partnering organization sites, their contact information, and their anticipated number and ages of boys for study participation;

a Serve as Boys Council Pilot Study communications liason between collaborating organizations and PSU evaluation team as is needed by PSU team and partnering organizations;

- Acknowledge PSU evaluation team on the Boys Council website: www.boyscouncil.com

- Explore and consider opportunities for further evaluation collaborations with $\mathrm{BC}$, following initial pilot study, based on experience, goals, and needs of both parties.

Department of Psychology, Portland State University:

1) Professor NAME:

Please Print Name

SIGNATURE

DATE

Boys Council, A Division of GCA/Tides:

Beth Hossfeld, MFT, Associate Director \& Co-Founder

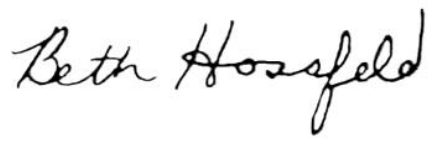

January 14, 2008 
APPENDIX B

Research Protocol

Protocol for Boys Council Research with Collaborating Program Partners

Ohio Department of Youth Services 


\section{Checklist - Research Study Steps for Collaborating Program Partners}

Use the following checklist as your guide when collaborating with Boys Council, A Division of GCA/Tides, on research.

- Read and sign the Confidentiality and Responsibility Form 1 for Facilitators and Program Supervisors and send form to: Boys Council Study, c/o: Eric Mankowski, PhD, Dep't of Psychology, Portland State University, P.O. Box 751, Portland, OR, 97207-0751; phone (503) 725-3901; e-mail: mankowskie@pdx.edu

Refer to the Boys Council Facilitator Guide to explain and administer the PreSurvey to youth.

- Share Form 2 Information Sheet for Boys and Young Men and Obtain Study Participation Consent Form 3 from participating youth. Make a photocopy of Form 3 and send originals to: Boys Council Study, c/o: Eric Mankowski, PhD, (same as above), keep photocopy in a locked cabinet in a confidential location.

Ensure that the young men's birthdates and last 3 digits of their DYS \#s are written on the Cover Page of their surveys.

- Keep attendance records for each youth for each Boys Council or group session.

Administer the Boys Council Survey again at the Post-Test and again at the Follow Up. If you are at Ohio River Valley, this survey includes the Boys Council Satisfaction Survey (page 9).

Ensure that the young men's birthdates and last 3 digits of their DYS \#s are written on the Cover Page at the Post-Test and at the Follow Up, so that their surveys can be matched together.

I Include the total number of group sessions attended by each youth on the Facilitator Questionnaire at the Post- Survey and Follow Up administrations.

- Send the Post-Test and Follow Up surveys by mail to Boys Council Study, c/o: Eric Mankowski, PhD, Dep't of Psychology, Portland State University, P.O. Box 751, Portland, OR, 97207-0751 upon completion.

Congratulate yourself on making an important contribution to knowledge of young men's experiences in Boys Council groups, and a job well done! 


\section{Boys Council Facilitator Guide} Instructions for Consent and Survey Administration and FAQs

To be consistent, please use the following script to introduce the study to the youth in a one-on-one setting and before they enter the group for the pre-test:

"You will soon be asked to participate in a study. The study is interested in learning about your experiences here at Ohio Youth Services. I will describe the study to you and will also give you a paper [The information sheet Form \#2 for young men] that explains the study that you can keep. The paper that I give you will be reviewed with you once again before you are asked to participate in the study. Between now and then, please take time to think about whether or not you want to take part in the study. Please do not tell me your decision now, but if you have any questions please ask. If you choose to participate, you will be asked to fill out a questionnaire once at the beginning of the study, 10 weeks later, and 10 weeks after that. In the end, it is up to you whether or not you want to be a part of the study. Even if you chose to participate at the beginning, you can stop at any time. I will now read the information sheet that will provide you with more information." 


\section{Form 1}

Confidentiality and Responsibility Form for Facilitator(s) and Program Supervisor

Boys Council and the Researchers value participant confidentiality. To ensure participant confidentiality, we ask that you sign the following agreement and return to researcher at address at bottom of page.

Agency: City/State:

By signing this confidentiality form, I agree to:

- Obtain all participating young men's consent forms

- Send the consent forms to PSU research team

- Refrain from reading or viewing any of the survey responses inside the cover sheet

- For Ohio River Valley:

Keep attendance at Boys Council meetings

Write the total number of sessions attended on the facilitator Questionnaire

- Mail consent forms and surveys in sealed envelope as they are completed to the PSU research team

\section{Facilitator Name}

Telephone:

Fax:

Facilitator Signature:

Date:

E-mail: (please print clearly)

Facilitator Name:

Telephone:

Fax:

Facilitator Signature: Date:

E-mail: (please print clearly)

\section{Program Supervisor Name:}

Telephone:

Fax:

Program

Supervisor Signature: Date:

E-mail: (please print clearly) 


\section{Upon completion, please send to:}

Boys Council Study, c/o: Eric Mankowski, PhD, Dep'† of Psychology,

Portland State University, P.O. Box 751, Portland, OR, 97207-0751. 


\section{Form 2 Information Sheet for Boys and Young Men}

\section{The Boys Council Study}

We are starting a research project and would like your help. Take the time to read this sheet and talk about it with the person giving you the survey.

\section{Why is this study being done?}

We want to understand whether Boys Council groups make a difference for the young men that participate.

\section{Why did you choose me?}

We want to survey everyone who participates, and also survey young men who may or may not join a Boys Council group.

\section{What will my involvement be?}

We would like you to complete surveys now, 10 weeks later, and again 10 weeks later (after the $20^{\text {th }}$ session). If you are interested, we may also ask to interview you or have you participate in a focus group.

\section{What will happen to the survey and interview data?}

The researchers will complete a report that will be shared with the Boys Council developers at GCA/Tides, your facilitator, any organizations that are interested in Boys Council, and your guardian(s), if they request it. Study results will be available to you, as well. Email support@boyscouncil.com to request a report.

\section{Who will know what answers I give?}

Only the researcher will see what answers you give on the survey.

S/he will not know who completed each survey because you will only report your birthdate. S/he will not be able to use any names when the results are reported.

\section{What if I choose not to take part?}

You may refuse to take part. If you do decide to complete a survey, you may stop at any time without giving a reason. Your participation will not affect your experience in Boys Council or any other services that you receive.

\section{What are the possible risks of participating in this study?}

The surveys may ask questions that you do not feel comfortable answering. In which case, you can skip that particular question or set of questions on the survey.

\section{What are the possible benefits of participating in this study?}

You may not personally benefit from participating in this study. However, through your participation, you may help us learn about improving the Boys Council program in the future. 
Thank you for reading this sheet and considering this study. If you have any questions, call:

Boys Council Study, c/o: Eric Mankowski, PhD, Dep't of Psychology, Portland State University, phone (503) 725-3901; e-mail: mankowskie@pdx.edu;

HSRRC in the Office of Research and Sponsored Projects (ORSP), 600 Unitus Bldg., Portland State University, Portland, OR, 97207. Phone: (503) 725-4288 


\section{Form 3 \\ Consent Form}

\section{BOYS COUNCIL Study Participant Consent Form}

Yes, I want to participate in the Boys Council study. I know I can change my mind at any time.

No, I do not want to participate in the Boys Council study.

Your Name:

Your Signature:

Date: Mo/Day/Year

120 


\section{Pre-Test Instructions}

Pre-tests should be completed before the first group. To be consistent, please use the following scripts and guidelines:

1. Make sure each youth has completed and returned a consent form before participating in the study. Make sure he has been given a copy of the information sheet and consent form for his own record.

2. Fill out the Facilitator Questionnaire to provide information about the curriculum and activities used, as well as, the weekly attendance of the young men in your group.

a. Place this completed questionnaire in the manila envelope that the youth will also return their surveys to.

3. Please refer to it as a survey while in the young men's presence - do not call it a test!

4. Use the following script before handing out the surveys:

"I am going to give you a survey to fill out and will ask you some questions about how you feel about yourself. Your participation may help us learn what kinds of programs are helpful to youth. I will read each question and each possible response, one by one. Some questions have to do with who you are and how you get along with other people, some have to do with how you handle problems, and some will ask you what you think about being a young man. The most important

thing to remember is to be honest! This is not a test, and there are no right or wrong answers - everyone is different, so everyone will have different answers. This survey is used with young men like yourself, as well as others from different types of programs and settings. Therefore, you may find that some questions are not relevant to you or your situation. If this is the case, please skip that question. This is your time to figure out what you think about yourself - not what other people think about you. If there are questions on the survey that you don't understand, please ask me to explain. Please wait at the bottom of each page for further instructions."

5. Provide each young man with a survey and ask them to make sure that the survey has 8 pages. Replace the survey with a complete one if necessary.

6. Read each question aloud, and then read the response options one at a time.

7. Repeat or answer questions from the young men as necessary. 
8. When the surveys are completed, ask them to return them directly to the manila envelope and have the last young man seal the envelope to ensure anonymity.

9. Thank them when they are all done, and tell them they all did a great job!

10. Ask if they have any questions about the survey.

11. Give or mail the sealed manila envelope containing the youth's completed surveys and your completed facilitator questionnaire to the PSU research team. 


\section{Post-Test and Follow-Up Instructions}

Post-tests are to be completed 10 weeks after the pre-survey or after the $10^{\text {th }}$ Boys Council session for youth at Ohio River Valley. The follow-up surveys are to be completed 10 weeks after post-test. To be consistent, please use the following scripts and guidelines:

1. Fill out the Facilitator Questionnaire to provide information about the curriculum and activities used, as well as, the attendance of the young men in your group.

a. Place this completed questionnaire in the manila envelope that the youth will also return their surveys to.

2. Please refer to it as a survey while in the young men's presence - do not call it a test!

3. Use the following script:

"Now we're going to do the end of the group survey and will ask you some questions about how you feel about yourself. Your participation may help us learn what kinds of programs are helpful to youth. I will read each question and each possible response, one by one. If you choose to read ahead on your own, you may do so. Just like the one we did in the beginning, some questions have to do with who you are and how you get along with other people, some have to do with how you handle problems, and some will ask you what you think about being a young man. The most important thing to remember is to be honest! Remember that this is not a test, and there are no right answers - everyone is different, so everyone will have different answers. This survey is used with young men like yourself, as well as others from different types of school programs and settings. Therefore, you may find that some questions are not relevant to you or your situation. If this is the case, please skip that question and continue on to the next one. This is your time to figure out what you have gotten out of this program and how it may have changed how you think or feel about yourself. Please wait at the bottom of each page for further instructions."

4. Provide each young man with a survey and ask them to make sure that the survey has 8 pages. Replace the survey with a complete one if necessary.

5. Read each question aloud, and then read the response options one at a time.

6. Repeat or answer questions from the young men as necessary. 
7. When the surveys are completed, ask them to return them directly to the manila envelope and have the last young man seal the envelope to ensure anonymity.

8. Thank them when they are all done, and tell them they all did a great job!

9. Ask if they have any questions about the survey.

10. Mail the surveys in the original sealed envelope to the PSU research team.

If you have any questions at all throughout this process, please contact us so that we can work with you to create the best outcomes for the young men in your group! 


\section{Ohio River Valley \\ Facilitator Questionnaire}

Instructions: For each group you facilitate, please fill out this questionnaire before the Pre-, Post-, and Follow-up evaluations and return it to the manila envelope that will contain your group member's surveys.

\begin{tabular}{l|l|}
\hline $\begin{array}{l}\text { Please circle the survey } \\
\text { that this questionnaire } \\
\text { corresponds with: }\end{array}$ & Pre Post Follow-up \\
\hline $\begin{array}{l}\text { Please check the } \\
\text { curriculum book that was } \\
\text { used with your group: }\end{array}$ & $\begin{array}{l}\text { Standing Together } \\
\text { Growing Healthy, Going Strong }\end{array}$ \\
\hline $\begin{array}{l}\text { If you used activities from a Legacy } \\
\text { multiple curriculum books, } \\
\text { please identify the activity } \\
\text { that was used from each } \\
\text { curriculum: }\end{array}$ & \\
\hline $\begin{array}{l}\text { How many total sessions } \\
\text { has this group } \\
\text { participated in? }\end{array}$ & \\
\hline
\end{tabular}

Please indicate the number of sessions attended and the date the boy joined your group:

Last 3 Digits of Number of sessions

DYS \# attended:

Start Date:

\begin{tabular}{|l|l|l|}
\hline & & \\
\hline & & \\
\hline & & \\
\hline & & \\
\hline & & \\
\hline & & \\
\hline & & \\
\hline
\end{tabular}




\section{Frequently Asked Questions}

Q. What should I do if a youth asks me the meaning of a question or a word on the survey?

A. Please feel free to answer questions about the meaning of words or questions.

Q. What should I say if a youth tells me he doesn't want to answer a question or if a certain question does not pertain to him?

A. Encourage the youth to skip the question that he doesn't want to answer or cannot answer, but to continue to answer the other questions.

Q. What if the youth want to know more about why they are taking these surveys?

A. This survey will help us learn whether Boys Council groups make a difference for you. 


\section{Ohio River Valley and Circleville Experimental Group}

\section{Facilitator Questionnaire}

The facilitator questionnaire should be completed twice, before administering the Post-Test and again before the Follow-Up.

Please complete a facilitator questionnaire for each of the groups that you facilitate.

First, indicate the survey this questionnaire is corresponding with, the curriculum and activities used, and how many total sessions your group has participated in up to that date. Second, enter the last 3 DYS digits for each participating member of your group and the number of sessions they have attended (this should be zero for the pre-survey (:)). Lastly, return this completed form to the manila envelope which will also be a storing place for the youth's surveys.

\section{Pre-Survey}

This 8-page survey (without the satisfaction survey) should be administered before the first Boys Council session.

Ask the members of your group to return the survey to the same manila envelope which contains the facilitator questionnaire. After the last youth completes his survey, ask him to seal the envelope to ensure confidentiality.

\section{Post-Survey}

The entire 9-page survey (with the satisfaction survey) should be administered after the 10th Boys Council session.

Follow the manila envelope instructions above.

\section{Follow-Up}

The entire 9-page survey (with the satisfaction survey) should be administered after the 20th Boys Council session or after 10 weeks have passed since the Post-Survey.

Follow the manila envelope instructions above. 


\section{Indian River and Cuyahoga Hills Control Group}

\section{Facilitator Questionnaire}

The facilitator questionnaire should be completed twice, before administering the Post-Test and again before the Follow-Up. Please complete a facilitator questionnaire for each of the groups that you facilitate.

First, indicate the survey this questionnaire is corresponding with, the curriculum and activities used, and how many total sessions your group has participated in up to that date. Second, enter the last 3 DYS digits for each participating member of your group and the number of sessions they have attended (this should be zero for the pre-survey (:)). Lastly, return this completed form to the manila envelope which will also be a storing place for the youth's surveys.

\section{Pre-Survey, Post-Survey and Follow-Up}

The same 8-page survey should be administered before the first group session, after the 10th group session, and after the 20th group session or after 10 weeks have passed since the Post-Test. Do not use the satisfaction survey.

Ask the members of your group to return the survey to the same manila envelope which contains the facilitator questionnaire. After the last youth completes his survey, ask him to seal the envelope to ensure confidentiality. 


\section{Checklist for Boys Council Facilitators}

There are many important aspects to the Boys Council facilitator's role that will play a crucial part in reaching your goals and the aims of the Boys Council program. This checklist is a tip sheet for your success!

- Be prepared by reading and reviewing the theme and activity plan prior to each session - gather all your materials in advance.

a Follow the 7-Step Basic Circle Format: 1) Opening ; 2) Theme Introduction; 3)Warm Up; 4) Check-in (using a talking piece); 5) Activity; 6) Reflection; and 7) Closing.

- Avoid giving advice: instead, ask open ended questions to promote critical thinking.

Develop group agreements with council members. Review and reinforce the agreements often with the group by asking them what they are doing well and what they'd like to improve upon. Offer leadership roles, such as "Council Keepers" to help safeguard the agreements.

- Explain your legal and ethical responsibilities to the group including your obligation as mandated reporters. Provide a clear and explicit policy upfront that lets young men know what to expect.

- Safeguard the Council. Make a commitment to your primary task: protecting the physical, emotional and social/cultural safety of the council environment.

- Manage problematic group dynamics with a strength-based approach. Show respect to each youth. Give young men the power and responsibility to share in the remedies and decisions, within age appropriate parameters.

a Normalize mistakes in the group. Humor, kindness, and clear expectations give young men the structure they need to get themselves back on track.

- Reinforce good behavior: Make sure to catch young men doing the good stuff! Name it and credit the young man/men when they are "on track" with one another.

Ensure that safe and respectful boundaries and norms are present when discussing topics of diversity.

- Know and access professionals for consultation and referrals as needs arise for yourself or the youth.

- Have fun! And be flexible! Don't be too attached to the Facilitator Guide agenda or your own agenda. Sometimes the young men will have more immediate issues they need to discuss. Put the decision out to the group; promote team decision-making

- Make adaptations as needed to best serve your council's needs. While the format of each session is important to maintain for consistency and predictability, the amount of time provided for each step of the format can be adapted. Likewise, if an activity can be implemented in a more conducive manner for the group, feel free to adapt. Older teen boys might be comfortable with a talking or 
brainstorming activity while younger boys might prefer to "act it out", for example.

- Contact Boys Council developers at any time during this study with questions regarding the Boys Council model, use of the Activity Guides, or group facilitation concerns. 707.794.9477 or support@boyscouncil.com

口 THANK YOU FOR MAKING A DIFFERENCE! 


\section{Selected Scales and Sources:}

1. Sections "A" and "B" - Questions specific for Boys Council, contributions by J. Roa and A. Irvine, Ceres Policy Research, Santa Cruz, CA, (2008).

2. Section "C" - Gang Membership, 4 items; measuring membership, intent to leave and attitudes about gang. Partially adapted from published scale. vi

3. Section "D" - Adolescent Masculine Identity in Relationships Scale, 12 items; measuring ideas about masculinity. Used with permission of author.iii

4. Section "E" - Sub-Survey of Modified Aggression Scale, 8 items; (1993) measures caring and cooperative behaviors; modified by Bosworth \& Espelage.

5. Section "F" - Ethnic Identity- Teen Conflict Survey, 4 items, (1995) Bosworth \& Espelage; measures ethnic pride and respect for differences.

6. Section "G" - Self-Efficacy Scale; 7 items. Prothrow-Stith, (1987), Additional Item developed by DeJong, Spiro, Brewer-Wilson, et al, 1992, Measures confidence in educational and career goals and avoiding fights.

7. Section "H" - Decision-Making Scale: 32 items: Jordan (unpublished manuscript). 
APPENDIX C

Survey Instrument

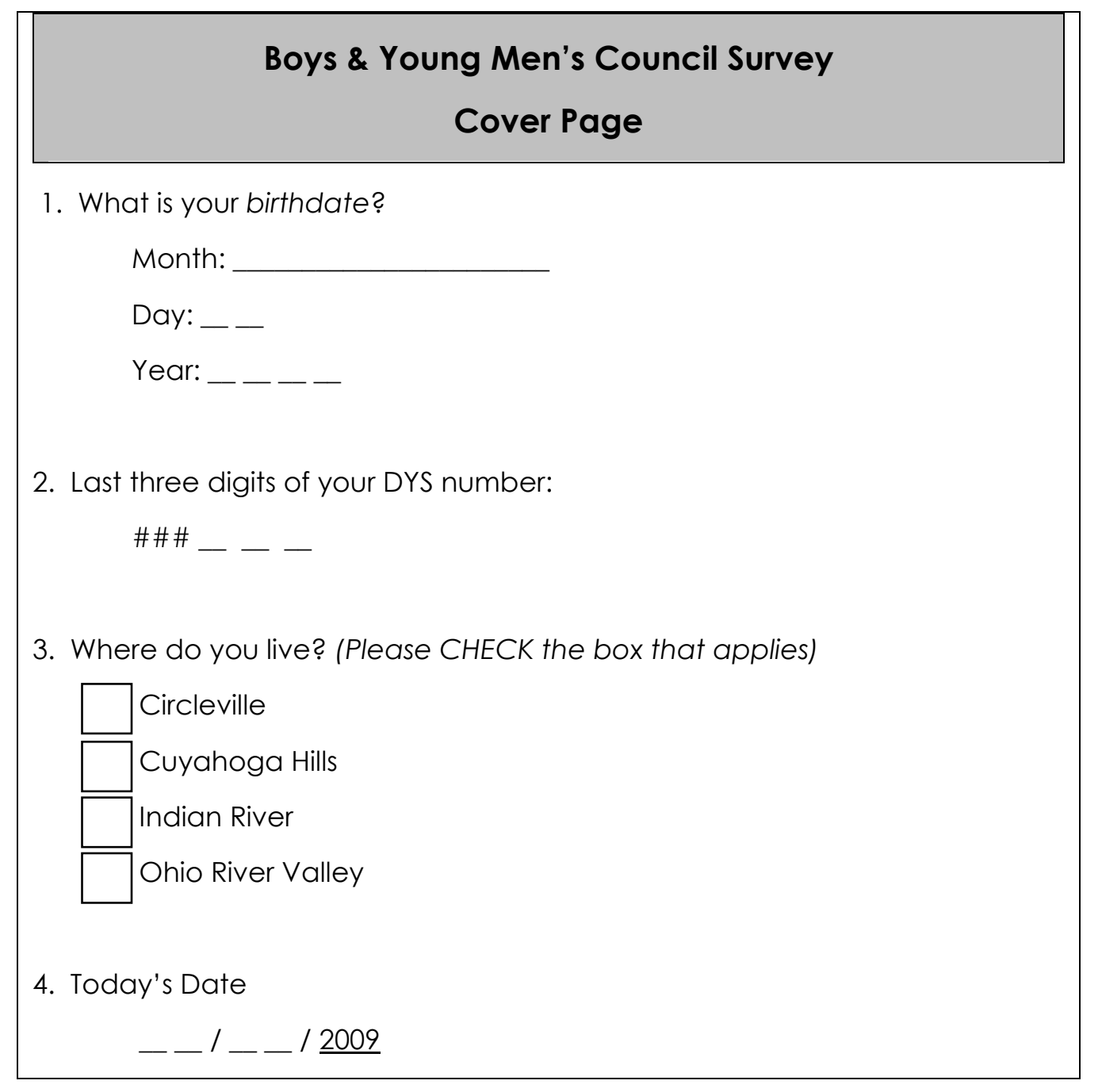

\section{PLEASE WAIT HERE FOR FURTHER INSTRUCTIONS}

(C) 2008 Boys Council, a Division of GCA/Tides

Permission to reproduce. Instruments included are public domain scales or authors have provided permission for this study. 


\section{Boys \& Young Men's Council Survey}

Please answer these questions about yourself and your life. Please be as honest as possible, and remember if you don't want to answer a question you don't have to. Please CIRCLE the answer that best applies to you. You can circle more than one answer.

\begin{tabular}{|c|c|}
\hline \multirow{2}{*}{ A 1. Please circle your age: } & 13 yrs $\quad 14$ yrs $\quad 15$ yrs $\quad 16$ yrs \\
\hline & 17 yrs 18 yrs 19 yrs 20 yrs 21 yrs \\
\hline $\begin{array}{l}\text { A2. Please circle your } \\
\text { race/ethnic identity: } \\
\text { (Please circle all that } \\
\text { apply. If you do not identify } \\
\text { with the categories provided, } \\
\text { please write in your response) }\end{array}$ & $\begin{array}{l}\text { African American Asian Latino } \\
\text { Native American White } \\
\text { Other: }\end{array}$ \\
\hline $\begin{array}{l}\text { A3. Who did you most } \\
\text { recently live with before you } \\
\text { came to Ohio Youth } \\
\text { Services? }\end{array}$ & $\begin{array}{l}\text { mother father mother and father } \\
\text { other family foster parent group home } \\
\text { Other: }\end{array}$ \\
\hline $\begin{array}{l}\text { A4. What languages do you } \\
\text { speak? }\end{array}$ & English \\
\hline
\end{tabular}

A5. Have you ever lived in a foster home or a group home?

\begin{tabular}{|l|l|l} 
yes & no & not sure
\end{tabular}

Please CIRCLE the number that shows how often you do the following things at school.

\begin{tabular}{|l|c|c|c|c|c|c|}
\hline & $\begin{array}{c}\text { Does } \\
\text { not } \\
\text { apply } \\
\text { to me } \\
\text { (N/A) }\end{array}$ & Never & $\begin{array}{c}\text { Not } \\
\text { Often }\end{array}$ & $\begin{array}{c}\text { Half } \\
\text { of } \\
\text { the } \\
\text { time }\end{array}$ & Often & Always \\
\hline $\begin{array}{l}\text { A6. I follow the rules at my } \\
\text { school. }\end{array}$ & 0 & 1 & 2 & 3 & 4 & 5 \\
\hline $\begin{array}{l}\text { A7. I feel good about my } \\
\text { school. }\end{array}$ & 0 & 1 & 2 & 3 & 4 & 5 \\
\hline $\begin{array}{l}\text { A8. I pay attention during } \\
\text { my classes. }\end{array}$ & 0 & 1 & 2 & 3 & 4 & 5 \\
\hline
\end{tabular}


Boys \& Young Men's Council Survey

Please CIRCLE the number that shows how much you agree or disagree with the statement.

\begin{tabular}{|c|c|c|c|c|c|c|}
\hline & & $\begin{array}{l}\text { Strongly } \\
\text { Disagree }\end{array}$ & Disagree & Agree & $\begin{array}{c}\text { Strongly } \\
\text { Agree }\end{array}$ & $\begin{array}{l}\text { Does } \\
\text { not } \\
\text { apply } \\
\text { to me } \\
\text { (N/A) }\end{array}$ \\
\hline B1. & $\begin{array}{l}\text { I am proud to be a } \\
\text { boy/young man. }\end{array}$ & 1 & 2 & 3 & 4 & 0 \\
\hline B2. & $\begin{array}{l}\text { I have things in } \\
\text { common with other } \\
\text { youth in my group. }\end{array}$ & 1 & 2 & 3 & 4 & 0 \\
\hline B3 & $\begin{array}{l}\text { I have good role } \\
\text { models in my life. }\end{array}$ & 1 & 2 & 3 & 4 & 0 \\
\hline B4. & $\begin{array}{l}\text { I share my feelings } \\
\text { with adults. }\end{array}$ & 1 & 2 & 3 & 4 & 0 \\
\hline B5. & $\begin{array}{l}\text { I am a good role } \\
\text { model to boys who } \\
\text { are younger than } \\
\text { me. }\end{array}$ & 1 & 2 & 3 & 4 & 0 \\
\hline
\end{tabular}

\begin{tabular}{|l|l|l|l|c|}
\hline Cl & I belong to a gang. & YES & NO & $\begin{array}{c}\text { I did in the past, but not } \\
\text { anymore }\end{array}$ \\
\hline
\end{tabular}

If you circled YES in question C1 above, please answer the following questions.

\begin{tabular}{|l|l|c|c|c|c|c|}
\hline & $\begin{array}{c}\text { Strong } \\
\text { ly } \\
\text { Disagr } \\
\text { ee }\end{array}$ & $\begin{array}{c}\text { Disagr } \\
\text { ee }\end{array}$ & $\begin{array}{c}\text { Agre } \\
\text { e }\end{array}$ & $\begin{array}{c}\text { Strong } \\
\text { ly } \\
\text { Agree }\end{array}$ & $\begin{array}{c}\text { Does not } \\
\text { apply to } \\
\text { me (N/A) }\end{array}$ \\
\hline C2 & $\begin{array}{l}\text { I plan to leave my gang } \\
\text { during the next two months. }\end{array}$ & 1 & 2 & 3 & 4 & 0 \\
\hline C3 & $\begin{array}{l}\text { I plan to leave my gang } \\
\text { during the next year. }\end{array}$ & 1 & 2 & 3 & 4 & 0 \\
\hline C4 & I like being in my gang. & 1 & 2 & 3 & 4 & 0 \\
\hline
\end{tabular}

PLEASE WAIT HERE FOR FURTHER INSTRUCTIONS 
Boys \& Young Men's Council Survey

Please CIRCLE the number that shows how much you agree or disagree with the statement

\begin{tabular}{|c|c|c|c|c|c|}
\hline & & $\begin{array}{l}\text { Strong } \\
\text { ly } \\
\text { Disagr } \\
\text { ee }\end{array}$ & $\begin{array}{l}\text { Disagr } \\
\text { ee }\end{array}$ & $\begin{array}{l}\text { Agre } \\
\mathrm{e}\end{array}$ & $\begin{array}{l}\text { Strong } \\
\text { ly } \\
\text { Agree }\end{array}$ \\
\hline D1. & $\begin{array}{l}\text { It's important for a guy to act like } \\
\text { nothing is wrong, even when } \\
\text { something is bothering him. }\end{array}$ & 1 & 2 & 3 & 4 \\
\hline D2. & $\begin{array}{l}\text { In a good dating relationship, the guy } \\
\text { gets his way most of the time. }\end{array}$ & 1 & 2 & 3 & 4 \\
\hline D3. & $\begin{array}{l}\text { I can respect a guy who backs down } \\
\text { from } \\
\text { a fight. }\end{array}$ & 1 & 2 & 3 & 4 \\
\hline D4. & It's ok for a guy to say no to sex. & 1 & 2 & 3 & 4 \\
\hline D5. & $\begin{array}{l}\text { Guys should not let it show when their } \\
\text { feelings are hurt. }\end{array}$ & 1 & 2 & 3 & 4 \\
\hline D6. & $\begin{array}{l}\text { A guy never needs to hit another guy } \\
\text { to } \\
\text { get respect }\end{array}$ & 1 & 2 & 3 & 4 \\
\hline D7 & $\begin{array}{l}\text { If a guy tells people his worries, he will } \\
\text { look weak. }\end{array}$ & 1 & 2 & 3 & 4 \\
\hline D8. & $\begin{array}{l}\text { I think it's important for a guy to go } \\
\text { after what he wants, even if it means } \\
\text { hurting } \\
\text { other people's feelings. }\end{array}$ & 1 & 2 & 3 & 4 \\
\hline D9. & $\begin{array}{l}\text { I think it's important for a guy to act } \\
\text { like he is sexually active even if he is } \\
\text { not. }\end{array}$ & 1 & 2 & 3 & 4 \\
\hline D10. & $\begin{array}{l}\text { I would be friends with a guy who is } \\
\text { gay. }\end{array}$ & 1 & 2 & 3 & 4 \\
\hline D11. & $\begin{array}{l}\text { It's embarrassing for a guy when he } \\
\text { needs } \\
\text { to ask for help. }\end{array}$ & 1 & 2 & 3 & 4 \\
\hline D12. & $\begin{array}{l}\text { I think it's important for a guy to talk } \\
\text { about } \\
\text { his feelings, even if people might } \\
\text { laugh at him. }\end{array}$ & 1 & 2 & 3 & 4 \\
\hline
\end{tabular}


Boys \& Young Men's Council Survey

This section asks about caring and cooperating. Please CIRCLE how many times you did each activity or task in the last 30 days.

\begin{tabular}{|c|c|c|c|c|c|}
\hline & In the last 30 days..... & Never & $\begin{array}{c}1 \text { or } \\
2 \\
\text { times } \\
\end{array}$ & $\begin{array}{l}3 \text { or } 4 \\
\text { times }\end{array}$ & $\begin{array}{l}5 \text { or } \\
\text { more } \\
\text { times } \\
\end{array}$ \\
\hline El. & I helped someone stay out of a fight. & 0 & $\begin{array}{c}1 \text { or } \\
2 \\
\text { times }\end{array}$ & $\begin{array}{l}3 \text { or } 4 \\
\text { times }\end{array}$ & $\begin{array}{l}5 \text { or } \\
\text { more } \\
\text { times }\end{array}$ \\
\hline E2. & $\begin{array}{l}\text { I told other kids how I felt when they } \\
\text { did something I liked. }\end{array}$ & 0 & $\begin{array}{c}1 \text { or } \\
2 \\
\text { times }\end{array}$ & $\begin{array}{l}3 \text { or } 4 \\
\text { times }\end{array}$ & $\begin{array}{l}5 \text { or } \\
\text { more } \\
\text { times }\end{array}$ \\
\hline E3. & I cooperated with others. & 0 & $\begin{array}{c}1 \text { or } \\
2 \\
\text { times }\end{array}$ & $\begin{array}{l}3 \text { or } 4 \\
\text { times }\end{array}$ & $\begin{array}{l}5 \text { or } \\
\text { more } \\
\text { times }\end{array}$ \\
\hline E4. & $\begin{array}{l}\text { I told other kids how I felt when they } \\
\text { upset me. }\end{array}$ & 0 & $\begin{array}{c}1 \text { or } \\
2 \\
\text { times }\end{array}$ & $\begin{array}{l}3 \text { or } 4 \\
\text { times }\end{array}$ & $\begin{array}{l}5 \text { or } \\
\text { more } \\
\text { times }\end{array}$ \\
\hline E5. & I protected someone from a "bully". & 0 & $\begin{array}{c}1 \text { or } \\
2 \\
\text { times }\end{array}$ & $\begin{array}{l}3 \text { or } 4 \\
\text { times }\end{array}$ & $\begin{array}{l}5 \text { or } \\
\text { more } \\
\text { times }\end{array}$ \\
\hline E6. & $\begin{array}{l}\text { I gave someone a } \\
\text { compliment. }\end{array}$ & 0 & $\begin{array}{c}1 \text { or } \\
2 \\
\text { times }\end{array}$ & $\begin{array}{l}3 \text { or } 4 \\
\text { times }\end{array}$ & $\begin{array}{l}5 \text { or } \\
\text { more } \\
\text { times }\end{array}$ \\
\hline E7. & I helped my peers solve a problem. & 0 & $\begin{array}{c}1 \text { or } \\
2 \\
\text { times }\end{array}$ & $\begin{array}{l}3 \text { or } 4 \\
\text { times }\end{array}$ & $\begin{array}{l}5 \text { or } \\
\text { more } \\
\text { times }\end{array}$ \\
\hline
\end{tabular}

PLEASE WAIT HERE FOR FURTHER INSTRUCTIONS 
Boys \& Young Men's Council Survey

This section asks about ethnic pride and respect for differences. Please CIRCLE the number that tells us how much you agree with the following statements.

\begin{tabular}{|l|l|c|c|c|c|c|}
\hline & Never & Rarely & $\begin{array}{c}\text { Sometime } \\
\text { s }\end{array}$ & Often & $\begin{array}{c}\text { Alway } \\
\text { s }\end{array}$ \\
\hline F1 & $\begin{array}{l}\text { I am proud to be a } \\
\text { member of my } \\
\text { racial/cultural group. }\end{array}$ & 0 & 1 & 2 & 3 & 4 \\
\hline $\begin{array}{l}\text { I am accepting of others } \\
\text { regardless of their race, } \\
\text { ethnicity, culture, or } \\
\text { religion. }\end{array}$ & 0 & 1 & 2 & 3 & 4 \\
\hline F3 & $\begin{array}{l}\text { I would help someone } \\
\text { regardless of their race. }\end{array}$ & 0 & 1 & 2 & 3 & 4 \\
\hline F4 & $\begin{array}{l}\text { I can get along with most } \\
\text { people. }\end{array}$ & 0 & 1 & 2 & 3 & 4 \\
\hline
\end{tabular}

This section asks about confidence in reaching goals and staying out of fights. Please CIRCLE the number that shows how much you agree or disagree with the statement.

\begin{tabular}{|l|l|c|c|c|c|}
\hline G1. & $\begin{array}{c}\text { Strongly } \\
\text { I will gragree } \\
\text { (or get my GED). }\end{array}$ & Disagree & Agree & $\begin{array}{c}\text { Strongly } \\
\text { Agree }\end{array}$ \\
\hline G2. & I will go to college. & 1 & 2 & 3 & 4 \\
\hline G3. & I will get a job I really want. & 1 & 2 & 3 & 4 \\
\hline G4. & $\begin{array}{l}\text { I am confident in my ability to } \\
\text { stay out of fights. }\end{array}$ & 1 & 2 & 3 & 4 \\
\hline G5. & $\begin{array}{l}\text { I don't need to fight because } \\
\text { there are other ways to deal } \\
\text { with anger. }\end{array}$ & 1 & 2 & 3 & 4 \\
\hline
\end{tabular}

PLEASE WAIT HERE FOR FURTHER INSTRUCTIONS 
Boys \& Young Men's Council Survey

People have different reasons for wanting to stop doing crime. Please CIRCLE the number that shows how important each reason is for you.

If I stop doing crime...

\begin{tabular}{|c|c|c|c|c|c|}
\hline & & $\begin{array}{c}\text { Not } \\
\text { Important }\end{array}$ & $\begin{array}{c}\text { Of Little } \\
\text { Importance }\end{array}$ & Important & $\begin{array}{c}\text { Very } \\
\text { Important }\end{array}$ \\
\hline $\mathrm{Hl}$. & $\begin{array}{l}\text { I will lose my tough } \\
\text { image. }\end{array}$ & 1 & 2 & 3 & 4 \\
\hline $\mathrm{H} 2$. & I will believe in myself. & 1 & 2 & 3 & 4 \\
\hline H3. & $\begin{array}{l}\text { The people I care } \\
\text { about will be proud of } \\
\text { me. }\end{array}$ & 1 & 2 & 3 & 4 \\
\hline $\mathrm{H} 4$. & $\begin{array}{l}\text { My associates will lose } \\
\text { respect for me. }\end{array}$ & 1 & 2 & 3 & 4 \\
\hline H5. & $\begin{array}{l}\text { I will have better } \\
\text { friends. }\end{array}$ & 1 & 2 & 3 & 4 \\
\hline H6. & $\begin{array}{l}\text { My family will respect } \\
\text { me. }\end{array}$ & 1 & 2 & 3 & 4 \\
\hline H7. & I will not feel a thrill. & 1 & 2 & 3 & 4 \\
\hline H8. & $\begin{array}{l}\text { I will be proud of } \\
\text { myself. }\end{array}$ & 1 & 2 & 3 & 4 \\
\hline H9. & $\begin{array}{l}\text { My family will be more } \\
\text { respected. }\end{array}$ & 1 & 2 & 3 & 4 \\
\hline H1O. & $\begin{array}{l}\text { My friends will not } \\
\text { respect me. }\end{array}$ & 1 & 2 & 3 & 4 \\
\hline $\mathrm{H} 11$. & $\begin{array}{l}\text { I will have more self- } \\
\text { respect. }\end{array}$ & 1 & 2 & 3 & 4 \\
\hline $\mathrm{H} 12$. & $\begin{array}{l}\text { The people I care } \\
\text { about will respect me } \\
\text { for "getting my act } \\
\text { together." }\end{array}$ & 1 & 2 & 3 & 4 \\
\hline H13. & $\begin{array}{l}\text { My family will not be } \\
\text { accepted by the } \\
\text { neighborhood. }\end{array}$ & 1 & 2 & 3 & 4 \\
\hline $\mathrm{H} 14$. & $\begin{array}{l}\text { I will feel better about } \\
\text { myself. }\end{array}$ & 1 & 2 & 3 & 4 \\
\hline $\mathrm{H} 15$. & $\begin{array}{l}\text { The people I care } \\
\text { about will trust me. }\end{array}$ & 1 & 2 & 3 & 4 \\
\hline
\end{tabular}

PLEASE WAIT HERE FOR FURTHER INSTRUCTIONS 
If I stop doing crime...

\begin{tabular}{|c|c|c|c|c|c|}
\hline & & $\begin{array}{c}\text { Not } \\
\text { Important }\end{array}$ & $\begin{array}{c}\text { Of Little } \\
\text { Importance }\end{array}$ & Important & $\begin{array}{c}\text { Very } \\
\text { Important }\end{array}$ \\
\hline H16. & $\begin{array}{l}\text { My associates will } \\
\text { lose a partner. }\end{array}$ & 1 & 2 & 3 & 4 \\
\hline H17. & I will feel safer. & 1 & 2 & 3 & 4 \\
\hline H18. & $\begin{array}{l}\text { The people I care } \\
\text { about will feel safe. }\end{array}$ & 1 & 2 & 3 & 4 \\
\hline H19. & $\begin{array}{l}\text { My friends will lose } \\
\text { a partner. }\end{array}$ & 1 & 2 & 3 & 4 \\
\hline H2O. & $\begin{array}{l}\text { I will not have to } \\
\text { worry about } \\
\text { getting arrested. }\end{array}$ & 1 & 2 & 3 & 4 \\
\hline H21. & $\begin{array}{l}\text { My family will be } \\
\text { closer. }\end{array}$ & 1 & 2 & 3 & 4 \\
\hline H22. & $\begin{array}{l}\text { I will not feel } \\
\text { powerful. }\end{array}$ & 1 & 2 & 3 & 4 \\
\hline $\mathrm{H} 23$. & I will be happier. & 1 & 2 & 3 & 4 \\
\hline H24. & $\begin{array}{l}\text { The people I care } \\
\text { about will feel } \\
\text { more comfortable } \\
\text { around me. }\end{array}$ & 1 & 2 & 3 & 4 \\
\hline $\mathrm{H} 25$. & $\begin{array}{l}\text { My family will have } \\
\text { more respect for } \\
\text { me. }\end{array}$ & 1 & 2 & 3 & 4 \\
\hline H26. & $\begin{array}{l}\text { I will not have to } \\
\text { look over my } \\
\text { shoulder. }\end{array}$ & 1 & 2 & 3 & 4 \\
\hline H27. & $\begin{array}{l}\text { I can help my } \\
\text { family. }\end{array}$ & 1 & 2 & 3 & 4 \\
\hline H28. & $\begin{array}{l}\text { The people I love } \\
\text { will be } \\
\text { embarrassed if I } \\
\text { got help. }\end{array}$ & 1 & 2 & 3 & 4 \\
\hline H29. & $\begin{array}{l}\text { I will feel proud of } \\
\text { myself. }\end{array}$ & 1 & 2 & 3 & 4 \\
\hline H3O. & $\begin{array}{l}\text { The people I } \\
\text { taught how to do } \\
\text { crime will not } \\
\text { respect me. }\end{array}$ & 1 & 2 & 3 & 4 \\
\hline H31. & $\begin{array}{l}\text { I can be part of my } \\
\text { neighborhood. }\end{array}$ & 1 & 2 & 3 & 4 \\
\hline
\end{tabular}




\begin{tabular}{|l|l|l|l|l|l|}
\hline H32. & $\begin{array}{l}\text { The people who } \\
\text { taught me how to } \\
\text { do crime will not } \\
\text { respect me. }\end{array}$ & 1 & 2 & 3 & 4 \\
\hline
\end{tabular}




\section{Boys \& Young Men's Council Satisfaction Survey For Post Survey and Follow-Up administrations only}

Please read the following statements and CIRCLE the number that represents how you felt when you were in Boys \& Young Men's Council.

\begin{tabular}{|l|l|c|c|c|c|}
\hline & & $\begin{array}{c}\text { Nev } \\
\text { er }\end{array}$ & $\begin{array}{c}\text { Sometim } \\
\text { es }\end{array}$ & $\begin{array}{c}\text { Usua } \\
\text { lly }\end{array}$ & $\begin{array}{c}\text { Alway } \\
\text { s }\end{array}$ \\
\hline S1. & $\begin{array}{l}\text { I could say what I was thinking in } \\
\text { Boys \& Young Men's Council. }\end{array}$ & 0 & 1 & 2 & 3 \\
\hline S2. & $\begin{array}{l}\text { I could trust Boys \& Young Men's } \\
\text { Council leaders. }\end{array}$ & 0 & 1 & 2 & 3 \\
\hline S3. & $\begin{array}{l}\text { People were fair in Boys \& Young } \\
\text { Men's Council. }\end{array}$ & 0 & 1 & 2 & 3 \\
\hline S4. & $\begin{array}{l}\text { Everyone respected me in Boys \& } \\
\text { Young Men's Council. }\end{array}$ & 0 & 1 & 2 & 3 \\
\hline S5. & $\begin{array}{l}\text { Boys \& Young Men's Council leaders } \\
\text { focused on what l'm good at. }\end{array}$ & 0 & 1 & 2 & 3 \\
\hline S6. & $\begin{array}{l}\text { Boys \& Young Men's Council was } \\
\text { worth my time. }\end{array}$ & 0 & 1 & 2 & 3 \\
\hline S7. & $\begin{array}{l}\text { People kept things confidential in } \\
\text { Boys \& Young Men's Council. }\end{array}$ & 0 & 1 & 2 & 3 \\
\hline
\end{tabular}

\begin{tabular}{|l|l|}
\hline S8. & What have you learned in Boys \& Young Men's Council? \\
\hline & \\
& \\
\hline S9. & What have you learned about being male? \\
\hline & \\
& \\
\hline
\end{tabular}




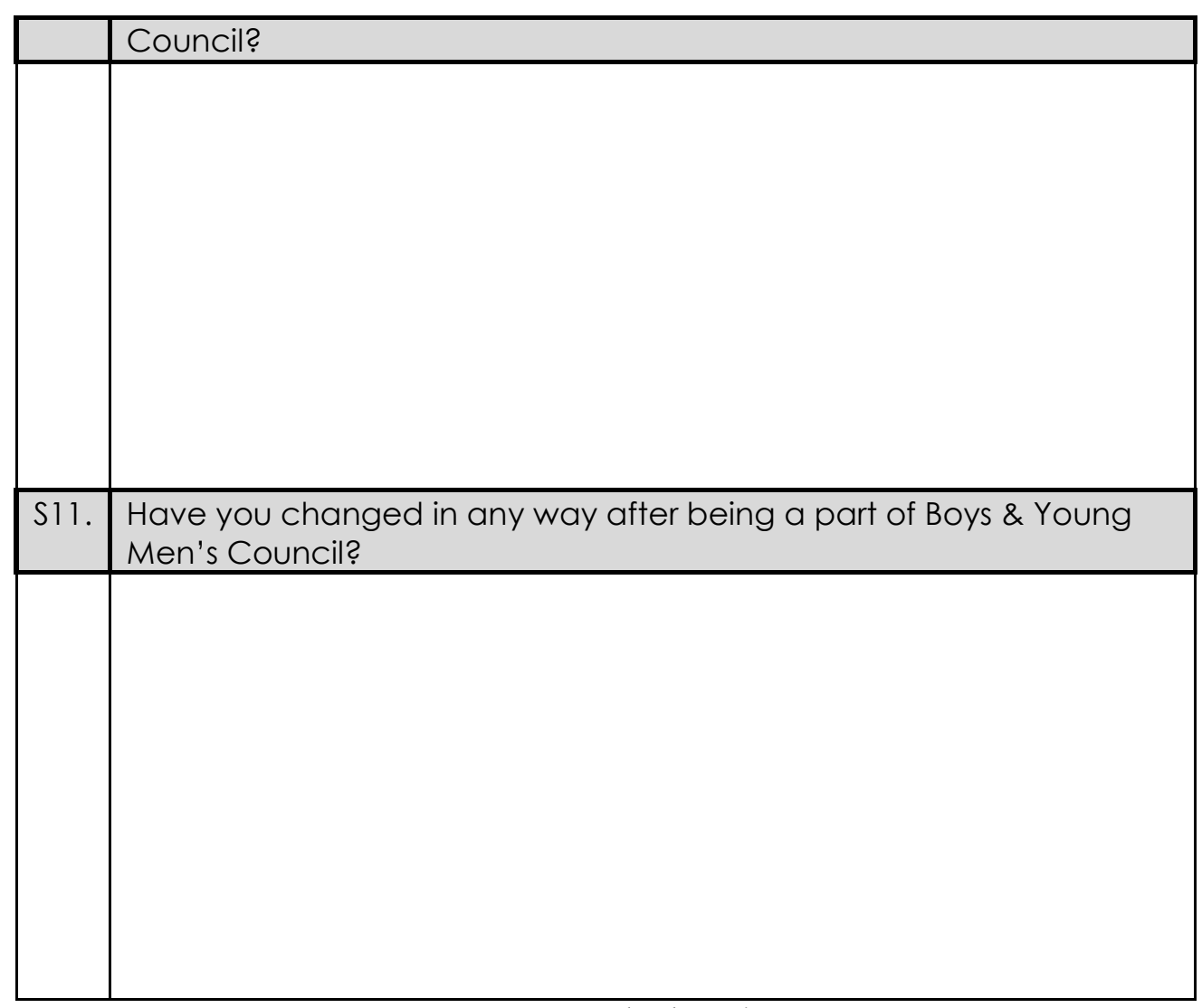

Thank you! 